\title{
Silver-Assisted Oxidative Isocyanide Insertion of Ethers: A Direct Approach to $\beta$-Carbonyl $\alpha$-Iminonitriles
}

\author{
Leiyang Zhao, ${ }^{\dagger}$ Bingxin Liu, ${ }^{\dagger}$ Qitao Tan, ${ }^{\dagger}$ Chang-Hua Ding, ${ }^{*, \dagger}$ and Bin $\mathrm{Xu}{ }^{*}, \dagger^{\dagger}$ \\ ${ }^{\dagger}$ Department of Chemistry, Innovative Drug Research Center, Shanghai University, \\ Shanghai 200444, China \\ ${ }^{\ddagger}$ State Key Laboratory of Organometallic Chemistry, Shanghai Institute of Organic \\ Chemistry, Chinese Academy of Sciences, Shanghai 200032, China
}

Tel/Fax: (+86) 21-66132830; E-mail: xubin@shu.edu.cn; dingchanghua@shu.edu.cn

\section{Supporting Information}

\section{CONTENTS:}

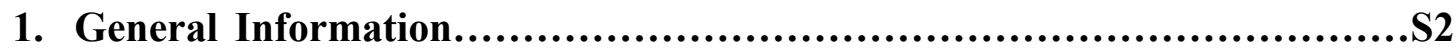

2. Synthesis and Characterization of tert-Butyl Ethers...........................S3

3. Synthesis and Characterization of Products.................................S6

4. Synthetic Applications of $\beta$-Carbonyl $\alpha$-Iminonitrile 2a....................S14

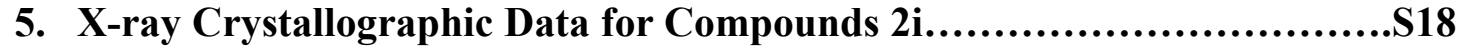

6. Mechanistic Studies....................................................S19

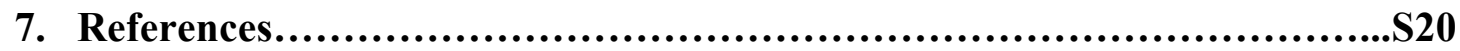

8. Copies of ${ }^{1} \mathrm{H},{ }^{13} \mathrm{C}$ and ${ }^{19} \mathrm{~F}$ NMR Spectra.....................................S21 


\section{General Information}

All reagents and metal catalysts were obtained from commercial sources without further purification, and commercially available solvents were purified before use. All new compounds were fully characterized. All melting points were taken on a WRS-1A or a WRS-1B Digital Melting Point Apparatus without correction. Infrared spectra were obtained using an AVATAR 370 FT-IR spectrometer. ${ }^{1} \mathrm{H},{ }^{13} \mathrm{C}$, and ${ }^{19} \mathrm{~F}$ NMR spectra were recorded with a Bruker AV-500 spectrometer operating at 500 $\mathrm{MHz}$ and $125 \mathrm{MHz}$, respectively, with chemical shift values being reported in ppm relative to chloroform $(\delta=7.26 \mathrm{ppm})$, acetone $(\delta=2.05 \mathrm{ppm})$ or TMS $(\delta=0.00 \mathrm{ppm})$ for ${ }^{1} \mathrm{H}$ NMR; chloroform $(\delta=77.16 \mathrm{ppm})$ or acetone $(\delta=29.84$ and $206.26 \mathrm{ppm})$ for ${ }^{13} \mathrm{C}$ NMR; and $\mathrm{C}_{6} \mathrm{~F}_{6}(\delta=-164.9 \mathrm{ppm})$ for ${ }^{19} \mathrm{~F}$ NMR. Mass spectra (MS) and high resolution mass (HRMS) spectra were recorded with an Agilent 5975C or JEOL AccuTOF-MS using an Electron impact (EI), Electrospray ionization (ESI), or direct analysis in real-time (DART) techniques. Silica gel plate GF254 were used for thin layer chromatography (TLC) and silica gel $\mathrm{H}$ or $300-400$ mesh were used for flash column chromatography. The starting materials tert-butyl ethers were all prepared according to the literature reported procedures, ${ }^{1-11}$ unless otherwise indicated. 


\section{Synthesis and Characterization of tert-Butyl Ethers}

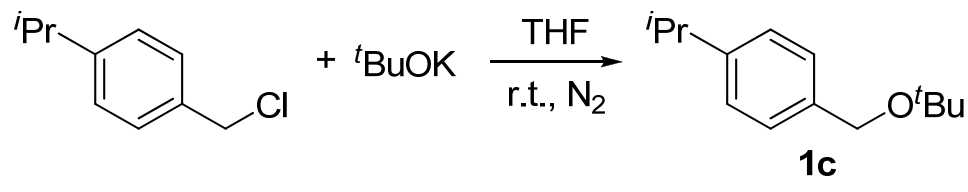

1-(tert-Butoxymethyl)-4-isopropylbenzene (1c): To a flask were added potassium tert-butoxide (1.68 g, $15.0 \mathrm{mmol}), 1$-(chloromethyl)-4-isopropylbenzene $(843.3 \mathrm{mg}, 5.0 \mathrm{mmol})$, and THF (10 $\mathrm{mL}$ ) under nitrogen atmosphere. The mixture was then stirred at room temperature for 10 hours until the reaction was completed (monitored by TLC). Then the mixture was poured into water ( 20 $\mathrm{mL})$. The mixture was extracted with ethyl acetate $(3 \times 30 \mathrm{~mL})$. The organic layer was collected, washed with brine and dried by $\mathrm{Na}_{2} \mathrm{SO}_{4}$. After filtration and concentration in vacuo, the resulting residue was purified by column chromatography on silica gel and eluted with petroleum ether to give $1 \mathrm{c}$ as a yellow oil liquid (749.8 $\mathrm{mg}, 73 \%$ yield). IR ( $\left.\mathrm{KBr}, \mathrm{cm}^{-1}\right): 3414,3127,2967,2033$, 1620, 1396, 1194, 1076, 816, 619; ${ }^{1} \mathrm{H}$ NMR (CDCl, $\left.500 \mathrm{MHz}\right): \delta 7.29$ (d, $\left.J=8.1 \mathrm{~Hz}, 2 \mathrm{H}\right), 7.20$ $(\mathrm{d}, J=8.0 \mathrm{~Hz}, 2 \mathrm{H}), 4.42(\mathrm{~s}, 2 \mathrm{H}), 2.93-2.88(\mathrm{~m}, 1 \mathrm{H}), 1.31(\mathrm{~s}, 9 \mathrm{H}), 1.25(\mathrm{~d}, J=8.1 \mathrm{~Hz}, 6 \mathrm{H}) ;{ }^{13} \mathrm{C}$ NMR ( $\left.\mathrm{CDCl}_{3}, 125 \mathrm{MHz}\right): \delta 147.9,137.3,127.7,126.5,73.4,64.2,34.0,27.8,24.2$; EI-MS $\mathrm{m} / \mathrm{z}$ (\%): 206.2 (25) [M] ${ }^{+}, 163.1$ (20), 133.1 (100), 117.1 (30), 107.0 (40), 91.1 (25), 79.1 (20); HRMS (EI) $m / z$ calcd for $\mathrm{C}_{14} \mathrm{H}_{22} \mathrm{O}[\mathrm{M}]^{+} 206.1671$, found 206.1670.

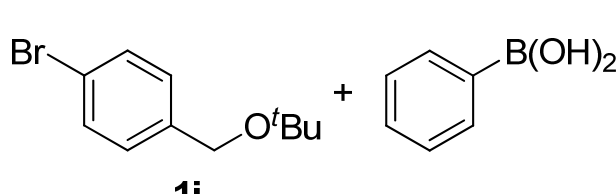

1i

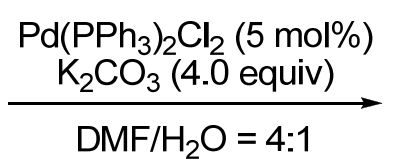

$\mathrm{N}_{2}, 80^{\circ} \mathrm{C}$<smiles>CCCCOc1ccc(-c2ccccc2)cc1</smiles>

$1 \mathrm{e}$

4-(tert-Butoxymethyl)-1,1'-biphenyl (1e): To a flask were added 1-bromo-4-(tert-butoxymethyl)benzene (486.3 mg, $2.0 \mathrm{mmol}$ ), phenylboronic acid (268.3 mg, $2.2 \mathrm{mmol}), \mathrm{Pd}\left(\mathrm{PPh}_{3}\right)_{2} \mathrm{Cl}_{2}$ (70.2 $\mathrm{mg}, 0.1 \mathrm{mmol}), \mathrm{K}_{2} \mathrm{CO}_{3}(1.11 \mathrm{~g}, 8.0 \mathrm{mmol})$, DMF $(8.0 \mathrm{~mL})$, and $\mathrm{H}_{2} \mathrm{O}(2.0 \mathrm{~mL})$ under nitrogen atmosphere. The mixture was then stirred at $80{ }^{\circ} \mathrm{C}$ for 18 hours until the reaction was completed (monitored by TLC). Then the mixture was poured into water $(20 \mathrm{~mL})$. The mixture was extracted with ethyl acetate $(3 \times 20 \mathrm{~mL})$. The organic layer was collected, washed with brine twice and dried by $\mathrm{Na}_{2} \mathrm{SO}_{4}$. After filtration and concentration in vacuo, the resulting residue was purified by column chromatography on silica gel and eluted with petroleum ether to give 1e as a white solid (403.2 mg, 84\% yield). m.p.: 29-30 ${ }^{\circ} \mathrm{C}$; IR $\left(\mathrm{KBr}, \mathrm{cm}^{-1}\right)$ : 3127, 2754, 2359, 1622, 1391 , 1192, 1076, 893, 827, 758, 696; ${ }^{1} \mathrm{H}$ NMR (CDCl 3 , $\left.500 \mathrm{MHz}\right): \delta 7.60-7.57$ (m, 4H), 7.44 (t, $J=7.4$ $\mathrm{Hz}, 4 \mathrm{H}), 7.35$ (t, $J=7.4 \mathrm{~Hz}, 1 \mathrm{H}), 4.51(\mathrm{~s}, 2 \mathrm{H}), 1.33(\mathrm{~s}, 9 \mathrm{H}) ;{ }^{13} \mathrm{C} \mathrm{NMR}\left(\mathrm{CDCl}_{3}, 125 \mathrm{MHz}\right): \delta 141.3$, 140.3, 139.1, 128.8, 128.0, 127.2, 73.6, 64.0, 27.9; EI-MS m/z (\%): 240.2 (30) [M] $]^{+}, 232.0$ (20), 215.0 (20), 184.1 (20), 167.1 (100), 165.1 (20), 155.1 (50), 152.1 (20); HRMS (EI) $\mathrm{m} / z$ calcd for $\mathrm{C}_{17} \mathrm{H}_{20} \mathrm{O}[\mathrm{M}]^{+}$240.1514, found 240.1510.<smiles>CCCCOCc1ccc(Br)cc1</smiles>

$1 \mathrm{i}$

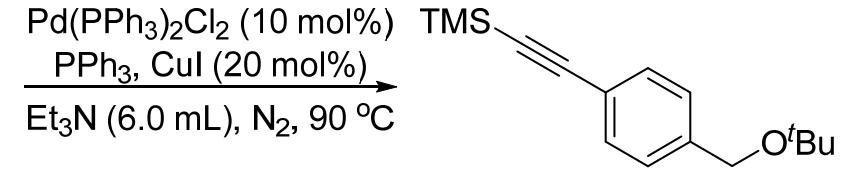

$1 \mathrm{k}$

((4-(tert-Butoxymethyl)phenyl)ethynyl)trimethylsilane $(\mathbf{1 k})$ : To a sealed tube were added 
1-bromo-4-(tert-butoxymethyl)benzene (486.3 mg, $2.0 \mathrm{mmol}), \mathrm{Pd}\left(\mathrm{PPh}_{3}\right)_{2} \mathrm{Cl}_{2}(140.4 \mathrm{mg}, 0.2$ mmol), $\mathrm{PPh}_{3}(104.9 \mathrm{mg}, 0.4 \mathrm{mmol}), \mathrm{CuI}(76.2 \mathrm{mg}, 0.4 \mathrm{mmol}$ ), ethynyltrimethylsilane ( $340 \mu \mathrm{L}, 2.4$ mmol), and $\mathrm{Et}_{3} \mathrm{~N}(6.0 \mathrm{~mL})$ under nitrogen atmosphere. The mixture was then stirred at $90{ }^{\circ} \mathrm{C}$ for 24 hours until the reaction was completed (monitored by TLC). Then the mixture was poured into water $(20 \mathrm{~mL})$. The mixture was extracted with ethyl acetate $(3 \times 30 \mathrm{~mL})$. The organic layer was collected, washed with brine twice and dried by $\mathrm{Na}_{2} \mathrm{SO}_{4}$. After filtration and concentration in vacuo, the resulting residue was purified by column chromatography on silica gel (petroleum ether/ethyl acetate $=100: 1, \mathrm{v} / \mathrm{v})$ to give $1 \mathbf{k}$ as a yellow solid $(424.9 \mathrm{mg}, 82 \%$ yield). m.p.: $51-52$ ${ }^{\circ} \mathrm{C}$; IR $\left(\mathrm{KBr}, \mathrm{cm}^{-1}\right): 3636,3414,2154,1616,1398,1248,1196,1082,866,758,617$; ${ }^{1} \mathrm{H}$ NMR $\left(\mathrm{CDCl}_{3}, 500 \mathrm{MHz}\right): \delta 7.42(\mathrm{~d}, J=8.2 \mathrm{~Hz}, 2 \mathrm{H}), 7.27(\mathrm{~d}, J=8.2 \mathrm{~Hz}, 2 \mathrm{H}), 4.44(\mathrm{~s}, 2 \mathrm{H}), 1.28(\mathrm{~s}, 9 \mathrm{H})$, 0.25 (s, 9H); ${ }^{13} \mathrm{C} \mathrm{NMR}\left(\mathrm{CDCl}_{3}, 125 \mathrm{MHz}\right): \delta 140.7,132.0,127.1,121.9,105.4,93.7,73.7,63.9$, 27.8, 0.2; EI-MS m/z (\%): 260.2 (65) [M] ${ }^{+}, 245.1$ (100), 204.1 (50), 175.1 (80), 159.1 (60), 73.0 (60), 57.1 (40); HRMS (EI) $\mathrm{m} / z$ calcd for $\mathrm{C}_{16} \mathrm{H}_{24} \mathrm{OSi}[\mathrm{M}]^{+} 260.1596$, found 260.1599.

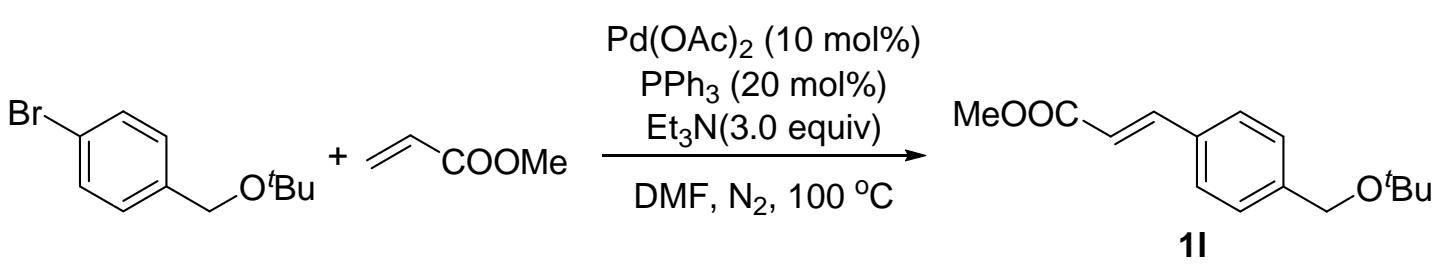

Methyl (E)-3-(4-(tert-butoxymethyl)phenyl)acrylate (11): To a sealed tube were added 1-bromo-4-(tert-butoxymethyl)benzene (486.3 mg, $2.0 \mathrm{mmol}), \mathrm{Pd}(\mathrm{OAc})_{2}(104.9 \mathrm{mg}, 0.2 \mathrm{mmol})$, $\mathrm{PPh}_{3}(104.9 \mathrm{mg}, 0.4 \mathrm{mmol})$, methyl acrylate $(272 \mu \mathrm{L}, 3.0 \mathrm{mmol}), \mathrm{Et}_{3} \mathrm{~N}(0.84 \mathrm{~mL}, 6.0 \mathrm{mmol})$, and DMF $(5.0 \mathrm{~mL})$ under nitrogen atmosphere. The mixture was then stirred at $100{ }^{\circ} \mathrm{C}$ for 36 hours until the reaction was completed (monitored by TLC). Then the mixture was poured into water ( 20 $\mathrm{mL})$. The mixture was extracted with ethyl acetate $(3 \times 30 \mathrm{~mL})$. The organic layer was collected, washed with brine twice and dried by $\mathrm{Na}_{2} \mathrm{SO}_{4}$. After filtration and concentration in vacuo, the resulting residue was purified by column chromatography on silica gel (petroleum ether/ethyl acetate $=50: 1, \mathrm{v} / \mathrm{v})$ to give 11 as a yellow solid $(285.4 \mathrm{mg}, 57 \%$ yield $)$. m.p.: $49-50{ }^{\circ} \mathrm{C}$; IR $(\mathrm{KBr}$, $\left.\mathrm{cm}^{-1}\right)$ : 3406, 3215, 3100, 2997, 2779, 2066, 1632, 1404, 1167, 1070, 893, 752, 621; ${ }^{1} \mathrm{H}$ NMR $\left(\mathrm{CDCl}_{3}, 500 \mathrm{MHz}\right): \delta 7.68(\mathrm{~d}, J=15.8 \mathrm{~Hz}, 1 \mathrm{H}), 7.49(\mathrm{~d}, J=8.2 \mathrm{~Hz}, 2 \mathrm{H}), 7.37(\mathrm{~d}, J=8.2 \mathrm{~Hz}, 2 \mathrm{H})$, $6.42(\mathrm{~d}, J=16.0 \mathrm{~Hz}, 1 \mathrm{H}), 4.46(\mathrm{~s}, 2 \mathrm{H}), 3.80(\mathrm{~s}, 3 \mathrm{H}), 1.29(\mathrm{~s}, 9 \mathrm{H}) ;{ }^{13} \mathrm{C} \mathrm{NMR}\left(\mathrm{CDCl}_{3}, 125 \mathrm{MHz}\right): \delta$ 167.7, 144.9, 142.7, 133.4, 128.2, 127.9, 117.4, 73.8, 63.8, 51.8, 27.8; EI-MS $m / z$ (\%): 248.1 (20) $[\mathrm{M}]^{+}, 192.1$ (30), 175.1 (100), 163.1 (98), 131.0 (30), 115.0 (80), 57.1 (30); HRMS (EI) $m / z$ calcd for $\mathrm{C}_{15} \mathrm{H}_{20} \mathrm{O}_{3}[\mathrm{M}]^{+}$248.1412, found 248.1406.
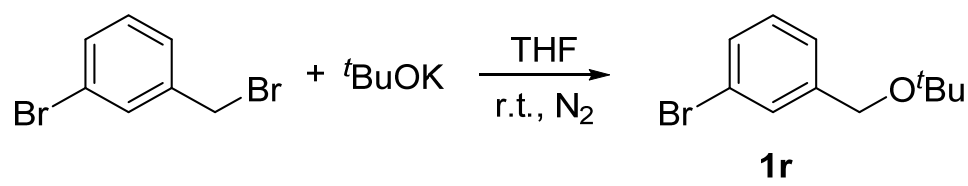

1-Bromo-3-(tert-butoxymethyl)benzene (1r): To a flask were added potassium tert-butoxide (1.12 g, $10.0 \mathrm{mmol})$, 1-bromo-3-(bromomethyl)benzene (1.25 g, $5.0 \mathrm{mmol})$, and THF (10 mL) under nitrogen atmosphere. The mixture was then stirred at room temperature for 12 hours until the reaction was completed (monitored by TLC). Then the mixture was poured into water $(20 \mathrm{~mL})$. The mixture was extracted with ethyl acetate $(3 \times 30 \mathrm{~mL})$. The organic layer was collected, washed with brine and dried by $\mathrm{Na}_{2} \mathrm{SO}_{4}$. After filtration and concentration in vacuo, the resulting residue 
was purified by column chromatography on silica gel (petroleum ether/ethyl acetate $=100: 1, \mathrm{v} / \mathrm{v}$ ) to give $1 \mathbf{r}$ as a yellow oil (658.3 mg, $54 \%$ yield). IR $\left(\mathrm{KBr}, \mathrm{cm}^{-1}\right): 3557,3416,3119,2974,2866$, 1618, 1470, 1398, 1236, 1192, 1069, 775, 611; ${ }^{1} \mathrm{H}$ NMR $\left(\mathrm{CDCl}_{3}, 500 \mathrm{MHz}\right): \delta 7.51$ (s, 1H), 7.37 $(\mathrm{d}, J=7.7 \mathrm{~Hz}, 1 \mathrm{H}), 7.25(\mathrm{~d}, J=6.1 \mathrm{~Hz}, 1 \mathrm{H}), 7.18(\mathrm{t}, J=7.8 \mathrm{~Hz}, 1 \mathrm{H}), 4.42(\mathrm{~s}, 2 \mathrm{H}), 1.29(\mathrm{~s}, 9 \mathrm{H})$; ${ }^{13} \mathrm{C} \mathrm{NMR}\left(\mathrm{CDCl}_{3}, 125 \mathrm{MHz}\right): \delta 142.4,130.5,130.3,129.9,125.9,122.6,73.8,63.5,27.8$; EI-MS $m / z(\%): m / z 242.0$ (1) [M] $]^{+}, 227.0$ (25), 171.0 (98), 169.0 (100), 107.0 (25), 90.0 (20), 57.1(25); HRMS (EI) $m / z$ calcd for $\mathrm{C}_{11} \mathrm{H}_{15} \mathrm{OBr}[\mathrm{M}]^{+} 242.0306$, found 242.0317 .

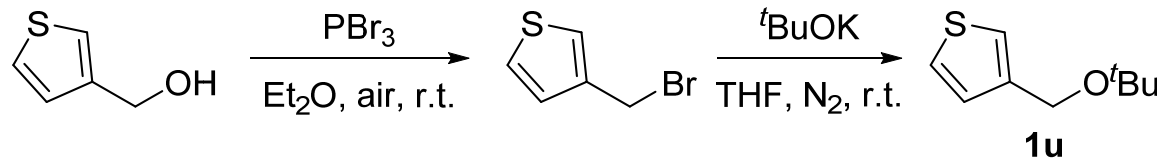

3-(tert-Butoxymethyl)thiophene (1u): To a flask were added thiophen-3-ylmethanol (570.9 mg, $5.0 \mathrm{mmol})$, and $\mathrm{Et}_{2} \mathrm{O}(10 \mathrm{~mL})$ under air atmosphere. Followed by the addition of $\mathrm{PBr}_{3}$, the mixture was then stirred at room temperature for 12 hours until the reaction was completed (monitored by TLC). After completion, the reaction was carefully quenched with $\mathrm{CH}_{3} \mathrm{OH}(1.5 \mathrm{~mL})$. Then the mixture was poured into water $(20 \mathrm{~mL})$. The mixture was extracted with ethyl acetate $(3 \times 20 \mathrm{~mL})$. The organic layer was collected, washed with brine and dried by $\mathrm{Na}_{2} \mathrm{SO}_{4}$. After filtration and concentration in vacuo, crude product 3-(bromomethyl)thiophene $(870.1 \mathrm{mg}, 98 \%$ yield) was obtained as a yellow oil liquid and used for the next step without purification.

To a flask were added potassium tert-butoxide (1.10 g, $9.8 \mathrm{mmol})$, crude 3-(bromomethyl)thiophene $(870.1 \mathrm{mg}, 4.9 \mathrm{mmol})$, and THF $(10 \mathrm{~mL})$ under nitrogen atmosphere. The mixture was then stirred at room temperature for 2 hours until the reaction was completed (monitored by TLC). Then the mixture was poured into water $(20 \mathrm{~mL})$. The mixture was extracted with ethyl acetate $(3 \times 20 \mathrm{~mL})$. The organic layer was collected, washed with brine and dried by $\mathrm{Na}_{2} \mathrm{SO}_{4}$. After filtration and concentration in vacuo, the resulting residue was purified by column chromatography on silica gel (petroleum ether/ethyl acetate $=100: 1, \mathrm{v} / \mathrm{v}$ ) to give $1 \mathbf{u}$ as a yellow oil (613.0 mg, 73\% yield). IR (KBr, cm $\left.{ }^{-1}\right): 3549,2731,2070,1630,1398,1194,1076,887,777$, 685, 623; ${ }^{1} \mathrm{H}$ NMR $\left(\mathrm{CDCl}_{3}, 500 \mathrm{MHz}\right): \delta$ 7.29-7.27 (m, $\left.1 \mathrm{H}\right), 7.20-7.19(\mathrm{~m}, 1 \mathrm{H}), 7.07-7.06(\mathrm{~m}, 1 \mathrm{H})$, $4.47(\mathrm{~s}, 2 \mathrm{H}), 1.29(\mathrm{~s}, 9 \mathrm{H}) ;{ }^{13} \mathrm{C} \mathrm{NMR}\left(\mathrm{CDCl}_{3}, 125 \mathrm{MHz}\right): \delta 141.1,127.2,125.8,121.8,73.5,60.0$, 27.8; EI-MS $m / z$ (\%): 170.1 (15) [M] ${ }^{+}, 141.1$ (10), 97.0 (100), 85.0 (10), 57.1 (10); HRMS (EI) $m / z$ calcd for $\mathrm{C}_{9} \mathrm{H}_{14} \mathrm{OS}[\mathrm{M}]^{+} 170.0765$, found 170.0770 .
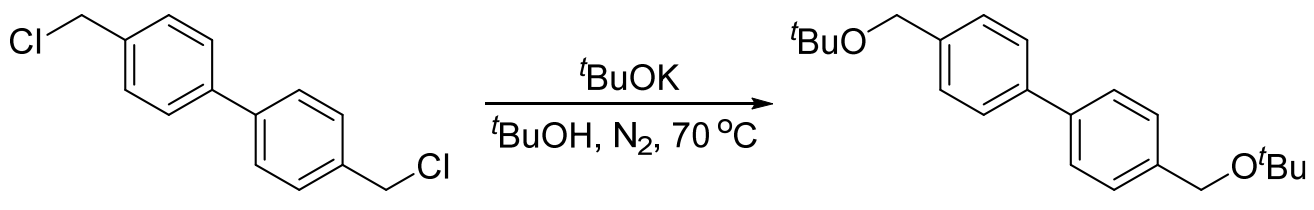

$1 \mathrm{v}$

4,4'-Bis(tert-butoxymethyl)-1,1'-biphenyl (1v): To a flask were added potassium tert-butoxide (2.24 g, $20 \mathrm{mmol}), 4,4^{\prime}$-bis(chloromethyl)-1,1'-biphenyl (1.25 g, $\left.5 \mathrm{mmol}\right)$, and ${ }^{t} \mathrm{BuOH}(25.0 \mathrm{~mL})$ under nitrogen atmosphere. The mixture was then stirred at $70{ }^{\circ} \mathrm{C}$ for 12 hours until the reaction was completed (monitored by TLC). Then the mixture was poured into water $(50 \mathrm{~mL})$. The mixture was extracted with ethyl acetate $(4 \times 50 \mathrm{~mL})$. The organic layer was collected, washed with brine and dried by $\mathrm{Na}_{2} \mathrm{SO}_{4}$. After filtration and concentration in vacuo, the resulting residue was purified by column chromatography on silica gel (petroleum ether/ethyl acetate $=100: 1, \mathrm{v} / \mathrm{v}$ ) to give $1 \mathbf{v}$ as a white solid $\left(419.5 \mathrm{mg}, 26 \%\right.$ yield). m.p.: $129-130{ }^{\circ} \mathrm{C}$; IR $\left(\mathrm{KBr}, \mathrm{cm}^{-1}\right): 3186,2359$, 
2066, 1624, 1393, 1190, 1078, 889, 802, 613; ${ }^{1} \mathrm{H}$ NMR $\left(\mathrm{CDCl}_{3}, 500 \mathrm{MHz}\right): \delta 7.54$ (d, $J=8.2 \mathrm{~Hz}$, $4 \mathrm{H}), 7.41(\mathrm{~d}, J=8.2 \mathrm{~Hz}, 4 \mathrm{H}), 4.48(\mathrm{~s}, 4 \mathrm{H}), 1.32(\mathrm{~s}, 18 \mathrm{H}) ;{ }^{13} \mathrm{C} \mathrm{NMR}\left(\mathrm{CDCl}_{3}, 125 \mathrm{MHz}\right): \delta 140.2$, 139.0, 128.0, 127.2, 73.6, 64.1, 27.9; EI-MS $m / z$ (\%): 326.2 (95) [M] $]^{+}, 253.2$ (100), 197.1 (40), 167.1 (50), 57.1 (40); HRMS (EI) $m / z$ calcd for $\mathrm{C}_{22} \mathrm{H}_{30} \mathrm{O}_{2}[\mathrm{M}]^{+}$326.2246, found 326.2239.

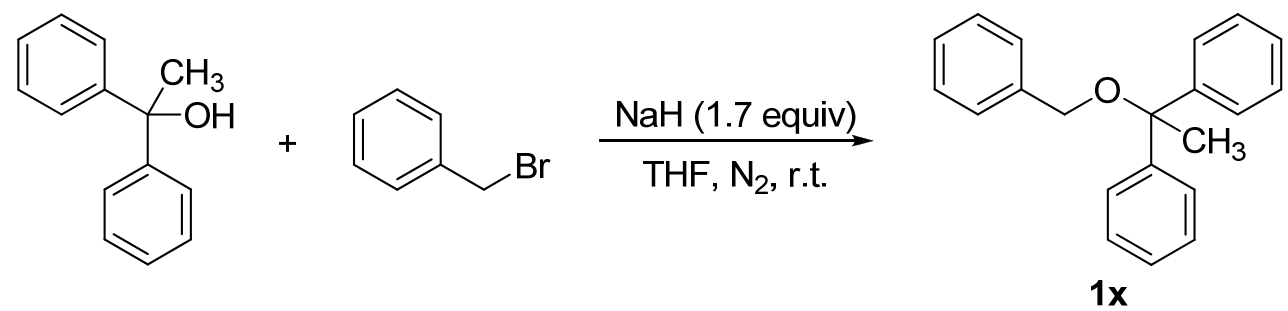

(1-(Benzyloxy)ethane-1,1-diyl)dibenzene (1x): To a flask, the mixture of 1,1-diphenylethanol $(1.98 \mathrm{~g}, 10.0 \mathrm{mmol})$ and THF $(20.0 \mathrm{~mL})$ was added $\mathrm{NaH}(60 \%, 680 \mathrm{mg}, 17.0 \mathrm{mmol})$ at $0{ }^{\circ} \mathrm{C}$ under nitrogen atmosphere. The mixture was stirred for 10 minutes was added benzyl bromide $(1.67 \mathrm{~mL}$, $14.0 \mathrm{mmol})$. The mixture was stirred at $25{ }^{\circ} \mathrm{C}$ for 12 hours until the reaction was completed (monitored by TLC). Then the mixture was poured into water $(50 \mathrm{~mL})$. The mixture was extracted with ethyl acetate $(4 \times 50 \mathrm{~mL})$. The organic layer was collected, washed with brine and dried by $\mathrm{Na}_{2} \mathrm{SO}_{4}$. After filtration and concentration in vacuo, the resulting residue was purified by column chromatography on silica gel and eluted with petroleum ether to give $\mathbf{1} \mathbf{x}$ as a white solid (429.3 mg, $15 \%$ yield). m.p.: $58-59{ }^{\circ} \mathrm{C} ;{ }^{1} \mathrm{H}$ NMR $\left(\mathrm{CDCl}_{3}, 500 \mathrm{MHz}\right): \delta 7.47$ (d, $\left.J=7.5 \mathrm{~Hz}, 4 \mathrm{H}\right), 7.42(\mathrm{~d}$, $J=7.4 \mathrm{~Hz}, 2 \mathrm{H}), 7.38(\mathrm{t}, J=7.4 \mathrm{~Hz}, 3 \mathrm{H}), 7.33-7.29(\mathrm{~m}, 4 \mathrm{H}), 7.27-7.24(\mathrm{~m}, 2 \mathrm{H}), 4.37$ (s, 2H), 2.00 (s, 3H); ${ }^{13} \mathrm{C} \mathrm{NMR}\left(\mathrm{CDCl}_{3}, 125 \mathrm{MHz}\right): \delta 146.8,139.7,128.4,128.2,127.3,127.2,127.0,126.9$, 81.2, 64.9, 26.1; LC-MS (ESI) $\mathrm{m} / z 311.1[\mathrm{M}+\mathrm{Na}]^{+}$; HRMS (ESI) $\mathrm{m} / z$ calcd for $\mathrm{C}_{21} \mathrm{H}_{20} \mathrm{ONa}$ $[\mathrm{M}+\mathrm{Na}]^{+} 311.1406$, found 311.1402 .

\section{Synthesis and Characterization of Products}

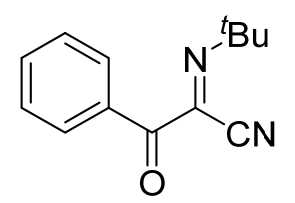

(E)-N-(tert-Butyl)-2-oxo-2-phenylacetimidoyl cyanide (2a): To a test tube, the mixture of DDQ (136.2 mg, $0.6 \mathrm{mmol}), \operatorname{AgOTf}(7.8 \mathrm{mg}, 0.03 \mathrm{mmol})$ and $\mathrm{PhCl}(3.0 \mathrm{~mL})$ was added $\mathbf{1 a}^{1}$ (53 $\mu \mathrm{L}, 0.3$ $\mathrm{mmol})$ and ${ }^{t} \mathrm{BuNC}(136 \mu \mathrm{L}, 1.2 \mathrm{mmol})$. The mixture was stirred at $80{ }^{\circ} \mathrm{C}$ for about $3 \mathrm{hrs}$ under $\mathrm{N}_{2}$ as monitored by TLC. Upon completion, the reaction mixture was cooled down to room temperature and concentrated under vacuum. The residue was purified by column chromatography on silica gel (petroleum ether/ethyl acetate $=100: 1, \mathrm{v} / \mathrm{v}$ ) to give 2a as a light yellow solid (52.7 mg, 82\% yield). m.p.: $34-35^{\circ} \mathrm{C}$; IR ( $\left.\mathrm{KBr}, \mathrm{cm}^{-1}\right): 3737,2975,2929,2860,2221,1676,1457,1282$, 1230, 915, 729; ${ }^{1} \mathrm{H}$ NMR ( $\left.\mathrm{CDCl}_{3}, 500 \mathrm{MHz}\right): \delta 8.05$ (d, $\left.J=7.3 \mathrm{~Hz}, 2 \mathrm{H}\right), 7.63(\mathrm{t}, J=7.3 \mathrm{~Hz}, 1 \mathrm{H})$, 7.48 (t, $J=7.8 \mathrm{~Hz}, 2 \mathrm{H}), 1.55$ (s, 9H); ${ }^{13} \mathrm{C}$ NMR $\left(\mathrm{CDCl}_{3}, 125 \mathrm{MHz}\right): \delta 186.3,136.6,134.3,133.4$, 131.2, 128.5, 110.8, 60.9, 29.1; LC-MS (ESI) $\mathrm{m} / z 215.1[\mathrm{M}+\mathrm{H}]^{+}$; HRMS (DART) $\mathrm{m} / z$ calcd for $\mathrm{C}_{13} \mathrm{H}_{15} \mathrm{~N}_{2} \mathrm{O}[\mathrm{M}+\mathrm{H}]^{+}$215.1179, found 215.1178.

\section{Gram-scale reaction}

To a bottle, the mixture of DDQ (4.54 g, $20.0 \mathrm{mmol})$, AgOTf (256.9 mg, $1.0 \mathrm{mmol})$ and $\mathrm{PhCl}$ 
$(100.0 \mathrm{~mL})$ was added $1 \mathrm{a}(1.64 \mathrm{~g}, 10.0 \mathrm{mmol})$ and ${ }^{t} \mathrm{BuNC}(4.53 \mathrm{~mL}, 40.0 \mathrm{mmol})$. The mixture was stirred at $80{ }^{\circ} \mathrm{C}$ for about $3 \mathrm{hrs}$ under $\mathrm{N}_{2}$ as monitored by TLC. Upon completion, the reaction mixture was cooled down to room temperature and concentrated under vacuum. The residue was purified by column chromatography on silica gel (petroleum ether/ethyl acetate $=100: 1, \mathrm{v} / \mathrm{v}$ ) to give 2a as a light yellow solid (1.61 g, $75 \%$ yield).<smiles>Cc1ccc(C(=O)/C(C#N)=N/[13CH]c2ccccc2Br)cc1</smiles>

(E)-N-(tert-Butyl)-2-oxo-2-(p-tolyl)acetimidoyl cyanide (2b): To a test tube, the mixture of DDQ (136.2 mg, $0.6 \mathrm{mmol})$, AgOTf (7.8 mg, $0.03 \mathrm{mmol})$ and $\mathrm{PhCl}(3.0 \mathrm{~mL})$ was added $\mathbf{1 b}^{2}(53.5$ $\mathrm{mg}, 0.3 \mathrm{mmol})$ and ${ }^{t} \mathrm{BuNC}(136 \mu \mathrm{L}, 1.2 \mathrm{mmol})$. The mixture was stirred at $80{ }^{\circ} \mathrm{C}$ for about $3 \mathrm{hrs}$ under $\mathrm{N}_{2}$ as monitored by TLC. Upon completion, the reaction mixture was cooled down to room temperature and concentrated under vacuum. The residue was purified by column chromatography on silica gel (petroleum ether/ethyl acetate $=100: 1, \mathrm{v} / \mathrm{v}$ ) to give $\mathbf{2 b}$ as a yellow solid $(57.6 \mathrm{mg}, 84 \%$ yield). m.p.: $60-61{ }^{\circ} \mathrm{C}$; IR ( $\left.\mathrm{KBr}, \mathrm{cm}^{-1}\right): 3679,2969,2361,1659,1551,1289,921,836,760 ;{ }^{1} \mathrm{H}$ NMR $\left(\mathrm{CDCl}_{3}, 500 \mathrm{MHz}\right): \delta 7.96(\mathrm{~d}, J=8.2 \mathrm{~Hz}, 2 \mathrm{H}), 7.27(\mathrm{~d}, J=7.9 \mathrm{~Hz}, 2 \mathrm{H}), 2.43(\mathrm{~s}, 3 \mathrm{H}), 1.55$ (s,

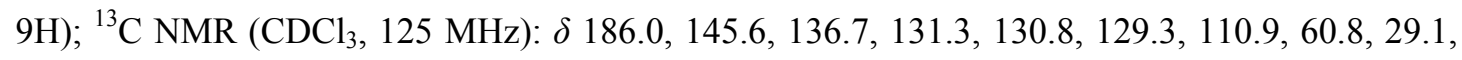
22.0; LC-MS (ESI) $m / z 229.1[\mathrm{M}+\mathrm{H}]^{+}$; HRMS (DART) $\mathrm{m} / z$ calcd for $\mathrm{C}_{14} \mathrm{H}_{17} \mathrm{~N}_{2} \mathrm{O}[\mathrm{M}+\mathrm{H}]^{+}$ 229.1335, found 229.1334 .<smiles>CCCCC(C)C</smiles>

(E)- $N$-(tert-butyl)-2-(4-isopropylphenyl)-2-oxoacetimidoyl cyanide (2c): To a test tube, the mixture of DDQ (136.2 mg, $0.6 \mathrm{mmol})$, AgOTf $(7.8 \mathrm{mg}, 0.03 \mathrm{mmol})$ and $\mathrm{PhCl}(3.0 \mathrm{~mL})$ was added $1 \mathrm{c}(61.9 \mathrm{mg}, 0.3 \mathrm{mmol})$ and ${ }^{t} \mathrm{BuNC}(136 \mu \mathrm{L}, 1.2 \mathrm{mmol})$. The mixture was stirred at $80{ }^{\circ} \mathrm{C}$ for about $3 \mathrm{hrs}$ under $\mathrm{N}_{2}$ as monitored by TLC. Upon completion, the reaction mixture was cooled down to room temperature and concentrated under vacuum. The residue was purified by column chromatography on silica gel (petroleum ether/ethyl acetate $=100: 1, \mathrm{v} / \mathrm{v}$ ) to give $\mathbf{2 c}$ as a yellow oil (61.6 mg, 80\% yield). IR ( $\left.\mathrm{KBr}, \mathrm{cm}^{-1}\right): 2968,2590,2220,1671,1606,1464,1284,1110,919,777$; ${ }^{1} \mathrm{H}$ NMR $\left(\mathrm{CDCl}_{3}, 500 \mathrm{MHz}\right): \delta 8.01(\mathrm{~d}, J=8.3 \mathrm{~Hz}, 2 \mathrm{H}), 7.33(\mathrm{~d}, J=8.2 \mathrm{~Hz}, 2 \mathrm{H}), 3.01-2.95(\mathrm{~m}$, $1 \mathrm{H}), 1.55(\mathrm{~s}, 9 \mathrm{H}), 1.28(\mathrm{~d}, J=7.0 \mathrm{~Hz}, 6 \mathrm{H}) ;{ }^{13} \mathrm{C} \mathrm{NMR}\left(\mathrm{CDCl}_{3}, 125 \mathrm{MHz}\right): \delta 185.9,156.2,131.5$, 131.2, 127.1, 126.8, 110.9, 60.8, 34.5, 29.2, 23.7; EI-MS m/z (\%): 256.2 (1) [M] $]^{+}, 147.1$ (100), 91.1 (60); HRMS (EI) $m / z$ calcd for $\mathrm{C}_{16} \mathrm{H}_{20} \mathrm{~N}_{2} \mathrm{O}[\mathrm{M}]^{+} 256.1576$, found 256.1585 .<smiles>CCCN=C(C#N)C(=O)c1ccc(C(C)(C)C)cc1</smiles>

(E)-N-(tert-Butyl)-2-(4-(tert-butyl)phenyl)-2-oxoacetimidoyl cyanide (2d): To a test tube, the mixture of DDQ (136.2 mg, $0.6 \mathrm{mmol})$, AgOTf $(7.8 \mathrm{mg}, 0.03 \mathrm{mmol})$ and $\mathrm{PhCl}(3.0 \mathrm{~mL})$ was added $\mathbf{1 d}^{3}(66.1 \mathrm{mg}, 0.3 \mathrm{mmol})$ and ${ }^{t} \mathrm{BuNC}(136 \mu \mathrm{L}, 1.2 \mathrm{mmol})$. The mixture was stirred at $80{ }^{\circ} \mathrm{C}$ for about $3 \mathrm{hrs}$ under $\mathrm{N}_{2}$ as monitored by TLC. Upon completion, the reaction mixture was cooled 
down to room temperature and concentrated under vacuum. The residue was purified by column chromatography on silica gel (petroleum ether/ethyl acetate $=100: 1, \mathrm{v} / \mathrm{v}$ ) to give $\mathbf{2 d}$ as a yellow oil (64.9 mg, 80\% yield). IR (KBr, cm $\left.{ }^{-1}\right): 3743,2968,2604,2218,1672,1605,1468,1285,920$, 861; $\left.{ }^{1} \mathrm{H} \mathrm{NMR} \mathrm{(CDCl}, 500 \mathrm{MHz}\right): \delta 8.02(\mathrm{~d}, J=8.5 \mathrm{~Hz}, 2 \mathrm{H}), 7.49(\mathrm{~d}, J=8.9 \mathrm{~Hz}, 2 \mathrm{H}), 1.56(\mathrm{~s}$,

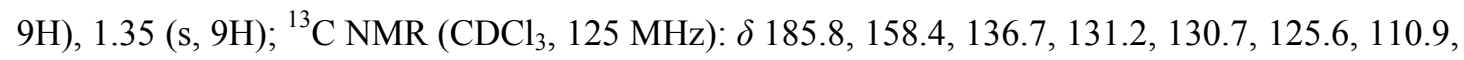
60.8, 35.4, 31.1, 29.2; LC-MS (ESI) $m / z 271.2[\mathrm{M}+\mathrm{H}]^{+}$; HRMS (DART) $m / z$ calcd for $\mathrm{C}_{17} \mathrm{H}_{23} \mathrm{~N}_{2} \mathrm{O}$ $[\mathrm{M}+\mathrm{H}]^{+}$271.1805, found 271.1803.<smiles>CCCN=C(C#N)C(=O)c1ccc(-c2ccccc2)cc1</smiles>

(E)-2-([1,1'-Biphenyl]-4-yl)- $\mathrm{N}$-(tert-butyl)-2-oxoacetimidoyl cyanide (2e): To a test tube, the mixture of DDQ (136.2 mg, $0.6 \mathrm{mmol})$, AgOTf $(7.8 \mathrm{mg}, 0.03 \mathrm{mmol})$ and $\mathrm{PhCl}(3.0 \mathrm{~mL})$ was added 1e $(72.10 \mathrm{mg}, 0.3 \mathrm{mmol})$ and ${ }^{t} \mathrm{BuNC}(136 \mu \mathrm{L}, 1.2 \mathrm{mmol})$. The mixture was stirred at $80{ }^{\circ} \mathrm{C}$ for about 3 hrs under $\mathrm{N}_{2}$ as monitored by TLC. Upon completion, the reaction mixture was cooled down to room temperature and concentrated under vacuum. The residue was purified by column chromatography on silica gel (petroleum ether/ethyl acetate $=100: 1, \mathrm{v} / \mathrm{v}$ ) to give $\mathbf{2 e}$ as a yellow solid (81.0 mg, 92\% yield). m.p.: 81-82 ${ }^{\circ} \mathrm{C}$; IR $\left(\mathrm{KBr}, \mathrm{cm}^{-1}\right): 2973,2217,1960,1660,1598,1285$, 1230, 920, 860, 749; ${ }^{1} \mathrm{H}$ NMR ( $\left.\mathrm{CDCl}_{3}, 500 \mathrm{MHz}\right): \delta 8.15(\mathrm{~d}, J=8.5 \mathrm{~Hz}, 2 \mathrm{H}), 7.71(\mathrm{~d}, J=8.5 \mathrm{~Hz}$, 2H), $7.64(\mathrm{~d}, J=7.5 \mathrm{~Hz}, 2 \mathrm{H}), 7.49(\mathrm{t}, J=7.6 \mathrm{~Hz}, 2 \mathrm{H}), 7.42(\mathrm{t}, J=7.4 \mathrm{~Hz}, 1 \mathrm{H}), 1.58(\mathrm{~s}, 9 \mathrm{H}) ;{ }^{13} \mathrm{C}$ NMR $\left(\mathrm{CDCl}_{3}, 125 \mathrm{MHz}\right): \delta 185.8,147.0,139.7,136.7,132.1,131.8,129.2,128.7,127.5,127.2$, 110.8, 60.9, 29.2; EI-MS $m / z$ (\%): 290.1 (1) [M] ${ }^{+}, 181.1$ (100), 152.1 (30); HRMS (EI) $m / z$ calcd for $\mathrm{C}_{19} \mathrm{H}_{18} \mathrm{~N}_{2} \mathrm{O}[\mathrm{M}]^{+} 290.1419$, found 290.1424 .

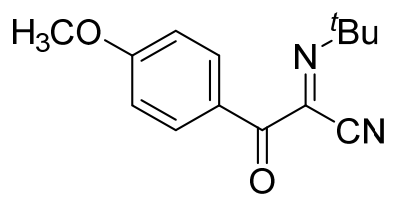

(E)-N-(tert-Butyl)-2-(4-methoxyphenyl)-2-oxoacetimidoyl cyanide (2f): To a test tube, the mixture of DDQ (136.2 mg, $0.6 \mathrm{mmol})$, AgOTf $(7.8 \mathrm{mg}, 0.03 \mathrm{mmol})$ and $\mathrm{PhCl}(3.0 \mathrm{~mL})$ was added $\mathbf{1} \mathbf{f}^{4}(58.3 \mathrm{mg}, 0.3 \mathrm{mmol})$ and ${ }^{t} \mathrm{BuNC}(136 \mu \mathrm{L}, 1.2 \mathrm{mmol})$. The mixture was stirred at $80{ }^{\circ} \mathrm{C}$ for about $3 \mathrm{hrs}$ under $\mathrm{N}_{2}$ as monitored by TLC. Upon completion, the reaction mixture was cooled down to room temperature and concentrated under vacuum. The residue was purified by column chromatography on silica gel (petroleum ether/ethyl acetate $=100: 1, \mathrm{v} / \mathrm{v}$ ) to give $\mathbf{2} \mathbf{f}$ as a yellow solid (58.6 mg, 80\% yield). m.p.: $38-39{ }^{\circ} \mathrm{C}$; IR ( $\left.\mathrm{KBr}, \mathrm{cm}^{-1}\right)$ : 3743, 2976, 2221, 2051, 1661, 1599 , 1263, 1175, 1113, 917, 843; ${ }^{1} \mathrm{H} \mathrm{NMR}\left(\mathrm{CDCl}_{3}, 500 \mathrm{MHz}\right): \delta 8.10$ (d, $\left.J=9.0 \mathrm{~Hz}, 2 \mathrm{H}\right), 6.95(\mathrm{~d}, J=$

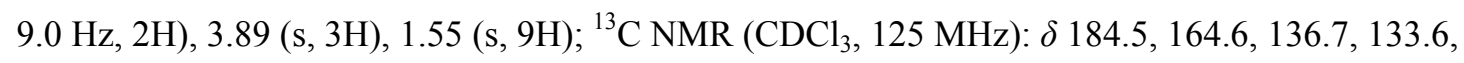
126.0, 113.9, 110.8, 60.5, 55.6, 29.0; EI-MS $m / z$ (\%): 244.1 (1) [M] $]^{+}, 135.0$ (100); HRMS (EI) $\mathrm{m} / \mathrm{z}$ calcd for $\mathrm{C}_{14} \mathrm{H}_{16} \mathrm{~N}_{2} \mathrm{O}_{2}[\mathrm{M}]^{+}$244.1212, found 244.1218 .

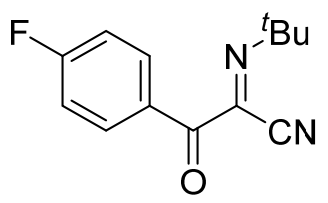

(E)-N-(tert-Butyl)-2-(4-fluorophenyl)-2-oxoacetimidoyl cyanide (2g): To a test tube, the 
mixture of DDQ (136.2 mg, $0.6 \mathrm{mmol}), \operatorname{AgOTf}(7.8 \mathrm{mg}, 0.03 \mathrm{mmol})$ and $\mathrm{PhCl}(3.0 \mathrm{~mL})$ was added $\mathbf{1 g}^{3}(54.7 \mathrm{mg}, 0.3 \mathrm{mmol})$ and ${ }^{t} \mathrm{BuNC}(136 \mu \mathrm{L}, 1.2 \mathrm{mmol})$. The mixture was stirred at $80{ }^{\circ} \mathrm{C}$ for about $3 \mathrm{hrs}$ under $\mathrm{N}_{2}$ as monitored by TLC. Upon completion, the reaction mixture was cooled down to room temperature and concentrated under vacuum. The residue was purified by column chromatography on silica gel (petroleum ether/ethyl acetate $=100: 1, \mathrm{v} / \mathrm{v}$ ) to give $\mathbf{2 g}$ as a white solid (55.5 mg, 80\% yield). m.p.: 49-50 ${ }^{\circ} \mathrm{C}$; IR $\left(\mathrm{KBr}, \mathrm{cm}^{-1}\right): 3739,2979,2931,2597,2211,1917$, 1678, 1598, 1294, 1232, 1114, 917, 846, 773; ${ }^{1} \mathrm{H}$ NMR ( $\left.\mathrm{CDCl}_{3}, 500 \mathrm{MHz}\right): \delta 8.13$ (m, 2H), 7.15 (t, $J=8.6 \mathrm{~Hz}, 2 \mathrm{H}), 1.55(\mathrm{~s}, 9 \mathrm{H}) ;{ }^{13} \mathrm{C} \mathrm{NMR}\left(\mathrm{CDCl}_{3}, 125 \mathrm{MHz}\right): \delta 184.7,167.5(\mathrm{~d}, J=257.8 \mathrm{~Hz})$, 136.6, $134.1(\mathrm{~d}, J=9.6 \mathrm{~Hz}), 129.8(\mathrm{~d}, J=2.7 \mathrm{~Hz}), 115.9(\mathrm{~d}, J=22.0 \mathrm{~Hz}), 110.6,60.9,29.1 ;{ }^{19} \mathrm{~F}$ NMR ( $\left.\mathrm{CDCl}_{3}, 470 \mathrm{MHz}\right): \delta-102.5$ (m, Ar-F); EI-MS m/z (\%): 232 (0.5) [M] $]^{+}, 217$ (1.3), 124 (7.9), 123 (100), 122 (0.3), 111 (0.6), 96 (2.1), 95 (27.7), 94 (1.8), 76 (0.9), 75 (9.3), 74 (1.2), 69 (1.5), 57 (20.6), 51 (1.0), 42 (2.1); HRMS (EI) $\mathrm{m} / z$ calcd for $\mathrm{C}_{13} \mathrm{H}_{13} \mathrm{~N}_{2} \mathrm{OF}[\mathrm{M}]^{+} 232.1012$, found 232.1016 .

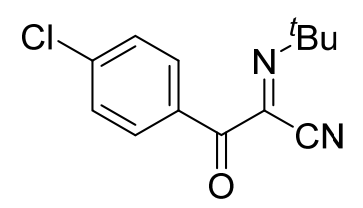

(E)-N-(tert-Butyl)-2-(4-chlorophenyl)-2-oxoacetimidoyl cyanide (2h): To a test tube, the mixture of DDQ (136.2 mg, $0.6 \mathrm{mmol})$, AgOTf $(7.8 \mathrm{mg}, 0.03 \mathrm{mmol})$ and $\mathrm{PhCl}(3.0 \mathrm{~mL})$ was added $\mathbf{1} \mathbf{h}^{5}$ (59.6 mg, $\left.0.3 \mathrm{mmol}\right)$ and ${ }^{t} \mathrm{BuNC}(136 \mu \mathrm{L}, 1.2 \mathrm{mmol})$. The mixture was stirred at $80{ }^{\circ} \mathrm{C}$ for about $3 \mathrm{hrs}$ under $\mathrm{N}_{2}$ as monitored by TLC. Upon completion, the reaction mixture was cooled down to room temperature and concentrated under vacuum. The residue was purified by column chromatography on silica gel (petroleum ether/ethyl acetate $=100: 1, \mathrm{v} / \mathrm{v}$ ) to give $\mathbf{2 h}$ as a white solid (59.8 mg, 80\% yield). m.p.: 89-90 ${ }^{\circ} \mathrm{C}$; IR $\left(\mathrm{KBr}, \mathrm{cm}^{-1}\right)$ : 3679, 2971, 2926, 2360, 1682, 1585, 1365, 1291, 1088, 918, 839, 768; ${ }^{1} \mathrm{H}$ NMR $\left(\mathrm{CDCl}_{3}, 500 \mathrm{MHz}\right): \delta 8.02(\mathrm{~d}, J=8.8 \mathrm{~Hz}, 2 \mathrm{H}), 7.45(\mathrm{~d}$, $J=8.7 \mathrm{~Hz}, 2 \mathrm{H}), 1.55$ (s, 9H); ${ }^{13} \mathrm{C} \mathrm{NMR}\left(\mathrm{CDCl}_{3}, 125 \mathrm{MHz}\right): \delta 185.1,141.0,136.5,132.5,131.8$, 128.9, 110.6, 61.0, 29.1; EI-MS m/z (\%): 248.1 (1) [M] $]^{+}, 139.0$ (100), 113.0 (30), 110.0 (95), 111 (0.6), 75.0 (30), 57.1 (40); HRMS (EI) $\mathrm{m} / z$ calcd for $\mathrm{C}_{13} \mathrm{H}_{13} \mathrm{~N}_{2} \mathrm{OCl}[\mathrm{M}]^{+}$248.0716, found 248.0719 .

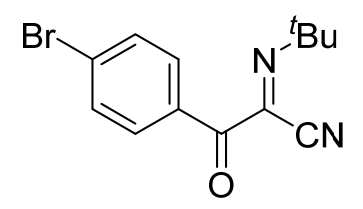

(E)-2-(4-Bromophenyl)- $N$-(tert-butyl)-2-oxoacetimidoyl cyanide (2i): To a test tube, the mixture of DDQ (136.2 mg, $0.6 \mathrm{mmol})$, AgOTf $(7.8 \mathrm{mg}, 0.03 \mathrm{mmol})$ and $\mathrm{PhCl}(3.0 \mathrm{~mL})$ was added $\mathbf{1 i}^{6}(72.9 \mathrm{mg}, 0.3 \mathrm{mmol})$ and ${ }^{t} \mathrm{BuNC}(136 \mu \mathrm{L}, 1.2 \mathrm{mmol})$. The mixture was stirred at $80{ }^{\circ} \mathrm{C}$ for about $3 \mathrm{hrs}$ under $\mathrm{N}_{2}$ as monitored by TLC. Upon completion, the reaction mixture was cooled down to room temperature and concentrated under vacuum. The residue was purified by column chromatography on silica gel (petroleum ether/toluene $=4: 1, \mathrm{v} / \mathrm{v}$ ) to give $\mathbf{2 i}$ as a white solid (62.4 mg, 71\% yield). m.p.: $101-102{ }^{\circ} \mathrm{C}$; IR $\left(\mathrm{KBr}, \mathrm{cm}^{-1}\right): 3445,3101,2977,2358,1680,1582,1472$, 1291, 1067, 917, 868, 839, 765; ${ }^{1} \mathrm{H}$ NMR $\left(\mathrm{CDCl}_{3}, 500 \mathrm{MHz}\right): \delta 7.93$ (d, $\left.J=8.4 \mathrm{~Hz}, 2 \mathrm{H}\right), 7.61(\mathrm{~d}$, $J=8.6 \mathrm{~Hz}, 2 \mathrm{H}), 1.54$ (s, 9H); ${ }^{13} \mathrm{C} \mathrm{NMR}\left(\mathrm{CDCl}_{3}, 125 \mathrm{MHz}\right): \delta 185.3,136.5,132.6,132.2,131.9$, 129.9, 110.5, 61.1, 29.1; LC-MS (ESI) $m / z 295.0\left[\mathrm{M}\left({ }^{81} \mathrm{Br}\right)+\mathrm{H}\right]^{+}, 293.0\left[\mathrm{M}\left({ }^{79} \mathrm{Br}\right)+\mathrm{H}\right]^{+}$; HRMS (DART) $m / z$ calcd for $\mathrm{C}_{13} \mathrm{H}_{14} \mathrm{~N}_{2} \mathrm{OBr}[\mathrm{M}+\mathrm{H}]^{+} 293.0284$, found 293.0283 . 
<smiles>CC(=O)Cc1ccc(C(=O)/C(C#N)=N/C(C)CC(C)(C)C)cc1</smiles>

Methyl (E)-4-(2-(tert-butylimino)-2-cyanoacetyl)benzoate (2j): To a test tube, the mixture of DDQ (204.3 mg, $0.9 \mathrm{mmol})$, AgOTf (7.8 $\mathrm{mg}, 0.03 \mathrm{mmol})$ and $\mathrm{PhCl}(3.0 \mathrm{~mL})$ was added $\mathbf{1} \mathbf{j}^{7}(66.7$ $\mathrm{mg}, 0.3 \mathrm{mmol})$ and ${ }^{t} \mathrm{BuNC}(170 \mu \mathrm{L}, 1.5 \mathrm{mmol})$. The mixture was stirred at $80{ }^{\circ} \mathrm{C}$ for about $3 \mathrm{hrs}$ under $\mathrm{N}_{2}$ as monitored by TLC. Upon completion, the reaction mixture was cooled down to room temperature and concentrated under vacuum. The residue was purified by column chromatography on silica gel (petroleum ether/ethyl acetate $=100: 1, \mathrm{v} / \mathrm{v}$ ) to give $\mathbf{2} \mathbf{j}$ as a yellow solid $(56.4 \mathrm{mg}, 69 \%$ yield). m.p.: $65-66{ }^{\circ} \mathrm{C}$; IR $\left(\mathrm{KBr}, \mathrm{cm}^{-1}\right)$ : 3852, 3740, 2978, 2219, 1727, 1683, 1514, 1281, 1199 , 1108, 918, 868, 737; ${ }^{1} \mathrm{H}$ NMR ( $\left.\mathrm{CDCl}_{3}, 500 \mathrm{MHz}\right): \delta 8.12(\mathrm{~d}, J=8.6 \mathrm{~Hz}, 2 \mathrm{H}), 8.08(\mathrm{~d}, J=8.6 \mathrm{~Hz}$, 2H), 3.96 (s, 3H), 1.55 (s, 9H); ${ }^{13} \mathrm{C} \mathrm{NMR}\left(\mathrm{CDCl}_{3}, 125 \mathrm{MHz}\right): \delta$ 186.0, 166.2, 137.0, 136.5, 134.6, 131.0, 129.5, 110.5, 61.2, 52.7, 29.1; EI-MS $m / z$ (\%): 272.1 (1) [M] $]^{+}, 164.0$ (40), 163.0 (100), 135.0 (40), 57.1 (30); HRMS (EI) $m / z$ calcd for $\mathrm{C}_{15} \mathrm{H}_{16} \mathrm{~N}_{2} \mathrm{O}_{3}[\mathrm{M}]^{+} 272.1161$, found 272.1166.

TMS<smiles>CC#Cc1ccc(C(=O)/C(C#N)=N/C(C)CC)cc1</smiles>

(E)-N-(tert-Butyl)-2-oxo-2-(4-((trimethylsilyl)ethynyl)phenyl)acetimidoyl cyanide (2k): To a test tube, the mixture of DDQ (136.2 $\mathrm{mg}, 0.6 \mathrm{mmol}), \operatorname{AgOTf}(7.8 \mathrm{mg}, 0.03 \mathrm{mmol})$ and $\mathrm{PhCl}(3.0$ $\mathrm{mL})$ was added $1 \mathbf{k}(78.1 \mathrm{mg}, 0.3 \mathrm{mmol})$ and ${ }^{t} \mathrm{BuNC}(136 \mu \mathrm{L}, 1.2 \mathrm{mmol})$. The mixture was stirred at $80{ }^{\circ} \mathrm{C}$ for about $3 \mathrm{hrs}$ under $\mathrm{N}_{2}$ as monitored by TLC. Upon completion, the reaction mixture was cooled down to room temperature and concentrated under vacuum. The residue was purified by column chromatography on silica gel (petroleum ether/ethyl acetate $=100: 1, \mathrm{v} / \mathrm{v}$ ) to give $\mathbf{2 k}$ as a yellow solid $\left(63.7 \mathrm{mg}, 68 \%\right.$ yield). m.p.: $70-71{ }^{\circ} \mathrm{C}$; IR $\left(\mathrm{KBr}, \mathrm{cm}^{-1}\right): 3437,2971,2217,2156$, $1931,1673,1630,1598,1285,1244,1114,864,765 ;{ }^{1} \mathrm{H} \mathrm{NMR}\left(\mathrm{CDCl}_{3}, 500 \mathrm{MHz}\right): \delta 7.99$ (d, $J=$ $8.5 \mathrm{~Hz}, 2 \mathrm{H}), 7.53(\mathrm{~d}, J=8.5 \mathrm{~Hz}, 2 \mathrm{H}), 1.54(\mathrm{~s}, 9 \mathrm{H}), 0.26(\mathrm{~s}, 9 \mathrm{H}) ;{ }^{13} \mathrm{C} \mathrm{NMR}\left(\mathrm{CDCl}_{3}, 125 \mathrm{MHz}\right): \delta$ $185.5,136.5,132.8,131.9,130.9,129.2,110.6,103.9,99.5,60.9,29.1,-0.1$; EI-MS $\mathrm{m} / z(\%)$ : 301.1 (1) [M] ${ }^{+}, 202.1$ (20), 201.1 (100), 158.1 (15); HRMS (EI) $\mathrm{m} / z$ calcd for $\mathrm{C}_{18} \mathrm{H}_{22} \mathrm{~N}_{2} \mathrm{OSi}[\mathrm{M}]^{+}$ 310.1501 , found 310.1497 .<smiles>CCCCN=C(C#N)C(=O)c1ccc(C=CC(C)C)cc1</smiles>

Methyl (E)-3-(4-((E)-2-(tert-butylimino)-2-cyanoacetyl)phenyl)acrylate (2I): To a test tube, the mixture of DDQ (136.2 mg, $0.6 \mathrm{mmol})$, AgOTf $(7.8 \mathrm{mg}, 0.03 \mathrm{mmol})$ and $\mathrm{PhCl}(3.0 \mathrm{~mL})$ was added 11 ( $74.5 \mathrm{mg}, 0.3 \mathrm{mmol})$ and ${ }^{t} \mathrm{BuNC}(136 \mu \mathrm{L}, 1.2 \mathrm{mmol})$. The mixture was stirred at $80{ }^{\circ} \mathrm{C}$ for about $3 \mathrm{hrs}$ under $\mathrm{N}_{2}$ as monitored by TLC. Upon completion, the reaction mixture was cooled down to room temperature and concentrated under vacuum. The residue was purified by column chromatography on silica gel (from petroleum ether/ethyl acetate $=30: 1$ to petroleum ether/ethyl acetate $=20: 1, \mathrm{v} / \mathrm{v})$ to give $2 \mathrm{I}$ as a yellow solid $(61.2 \mathrm{mg}, 68 \%$ yield $)$. m.p.: $97-98{ }^{\circ} \mathrm{C}$; $\mathrm{IR}(\mathrm{KBr}$, $\left.\mathrm{cm}^{-1}\right): 3846,3742,3413,2981,2218,1934,1718,1654,1605,1301,1200,915,836,771 ;{ }^{1} \mathrm{H}$ 
NMR $\left(\mathrm{CDCl}_{3}, 500 \mathrm{MHz}\right): \delta 8.07(\mathrm{~d}, J=8.0 \mathrm{~Hz}, 2 \mathrm{H}), 7.70(\mathrm{~d}, J=16.0 \mathrm{~Hz}, 1 \mathrm{H}), 7.60(\mathrm{~d}, J=8.2 \mathrm{~Hz}$, $2 \mathrm{H}), 6.54(\mathrm{~d}, J=16.0 \mathrm{~Hz}, 1 \mathrm{H}), 3.82(\mathrm{~s}, 3 \mathrm{H}), 1.55$ (s, 9H); ${ }^{13} \mathrm{C} \mathrm{NMR}\left(\mathrm{CDCl}_{3}, 125 \mathrm{MHz}\right): \delta 185.5$, $166.9,143.1,139.7,136.6,134.5,131.7,127.9,121.2,110.6,61.0,52.1,29.1$; EI-MS $m / z(\%)$ : 298.1 (1) $[\mathrm{M}]^{+}, 190.1$ (10), 189.1 (100), 183.1 (10), 102.0 (10); HRMS (EI) $\mathrm{m} / \mathrm{z}$ calcd for $\mathrm{C}_{17} \mathrm{H}_{18} \mathrm{~N}_{2} \mathrm{O}_{3}[\mathrm{M}]^{+}$298.1317, found 298.1311.

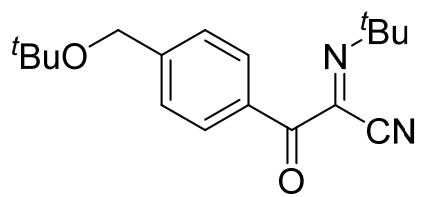

(E)-2-(4-(tert-Butoxymethyl)phenyl)- $\mathrm{N}$-(tert-butyl)-2-oxoacetimidoyl cyanide (2m): To a test tube, the mixture of DDQ (272.4 mg, $1.2 \mathrm{mmol}), \operatorname{AgOTf}(15.6 \mathrm{mg}, 0.06 \mathrm{mmol})$ and $\mathrm{PhCl}(3.0 \mathrm{~mL})$ was added $1 \mathbf{m}^{8}(75.1 \mathrm{mg}, 0.3 \mathrm{mmol})$ and ${ }^{t} \mathrm{BuNC}(272 \mu \mathrm{L}, 2.4 \mathrm{mmol})$. The mixture was stirred at $80{ }^{\circ} \mathrm{C}$ for about $3 \mathrm{hrs}$ under $\mathrm{N}_{2}$ as monitored by TLC. Upon completion, the reaction mixture was cooled down to room temperature and concentrated under vacuum. The residue was purified by column chromatography on silica gel (from petroleum ether/ethyl acetate $=100: 1$ to petroleum ether/ethyl acetate $=50: 1, \mathrm{v} / \mathrm{v})$ to give $\mathbf{2 m}$ as a yellow solid $\left(51.9 \mathrm{mg}, 57 \%\right.$ yield). m.p.: $97-98{ }^{\circ} \mathrm{C}$; IR $\left(\mathrm{KBr}, \mathrm{cm}^{-1}\right): 3427,2972,2548,2217,1672,1609,1371,1236,1080,915,761 ;{ }^{1} \mathrm{H}$ NMR $\left(\mathrm{CDCl}_{3}, 500 \mathrm{MHz}\right): \delta 8.01(\mathrm{~d}, J=8.6 \mathrm{~Hz}, 2 \mathrm{H}), 7.45(\mathrm{~d}, J=8.8 \mathrm{~Hz}, 2 \mathrm{H}), 4.52(\mathrm{~s}, 2 \mathrm{H}), 1.54(\mathrm{~s}, 9 \mathrm{H})$, $1.30(\mathrm{~s}, 9 \mathrm{H}) ;{ }^{13} \mathrm{C} \mathrm{NMR}\left(\mathrm{CDCl}_{3}, 125 \mathrm{MHz}\right): \delta 186.1,147.1,136.7,132.2,131.3,127.0,110.8,74.0$, 63.6, 60.8, 29.2, 27.8; LC-MS (ESI) $m / z 301.2[\mathrm{M}+\mathrm{H}]^{+}$; HRMS (DART) $\mathrm{m} / z$ calcd for $\mathrm{C}_{18} \mathrm{H}_{25} \mathrm{~N}_{2} \mathrm{O}_{2}$ $[\mathrm{M}+\mathrm{H}]^{+}$301.1911, found 301.1910.

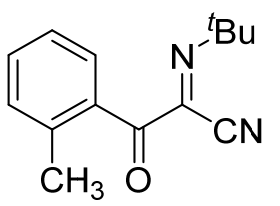

(E)-N-(tert-butyl)-2-oxo-2-(o-tolyl)acetimidoyl cyanide (2n): To a test tube, the mixture of DDQ (136.2 mg, $0.6 \mathrm{mmol})$, AgOTf (7.8 mg, $0.03 \mathrm{mmol})$ and $\mathrm{PhCl}(3.0 \mathrm{~mL})$ was added $\mathbf{1} \mathbf{n}^{2}(53.5 \mathrm{mg}$, $0.3 \mathrm{mmol})$ and ${ }^{t} \mathrm{BuNC}(136 \mu \mathrm{L}, 1.2 \mathrm{mmol})$. The mixture was stirred at $80{ }^{\circ} \mathrm{C}$ for about $3 \mathrm{hrs}$ under $\mathrm{N}_{2}$ as monitored by TLC. Upon completion, the reaction mixture was cooled down to room temperature and concentrated under vacuum. The residue was purified by column chromatography on silica gel (petroleum ether/ethyl acetate $=100: 1, \mathrm{v} / \mathrm{v}$ ) to give $\mathbf{2 n}$ as a white solid $(57.7 \mathrm{mg}, 84 \%$ yield). m.p.: $56-57{ }^{\circ} \mathrm{C}$; IR $\left(\mathrm{KBr}, \mathrm{cm}^{-1}\right): 3696,2979,2208,1679,1603,1271,1204,915,749 ;{ }^{1} \mathrm{H}$ NMR (CDCl $3,500 \mathrm{MHz}): \delta 7.51(\mathrm{~d}, J=7.7 \mathrm{~Hz}, 1 \mathrm{H}), 7.43(\mathrm{t}, J=7.4 \mathrm{~Hz}, 1 \mathrm{H}), 7.28-7.23(\mathrm{~m}, 2 \mathrm{H})$, $2.46(\mathrm{~s}, 3 \mathrm{H}), 1.49(\mathrm{~s}, 9 \mathrm{H}) ;{ }^{13} \mathrm{C} \mathrm{NMR}\left(\mathrm{CDCl}_{3}, 125 \mathrm{MHz}\right): \delta 189.9,139.8,137.6,133.5,132.5$, 131.8, 131.5, 125.2, 110.8, 60.7, 29.1, 21.2; LC-MS (ESI) $\mathrm{m} / \mathrm{z} 229.1[\mathrm{M}+\mathrm{H}]^{+}$; HRMS (DART) $\mathrm{m} / \mathrm{z}$ calcd for $\mathrm{C}_{14} \mathrm{H}_{17} \mathrm{~N}_{2} \mathrm{O}[\mathrm{M}+\mathrm{H}]^{+} 229.1335$, found 229.1334 .<smiles>CC(C)CN=C(C#N)C(=O)c1ccccc1Br</smiles>

(E)-2-(2-Bromophenyl)- $N$-(tert-butyl)-2-oxoacetimidoyl cyanide (20): To a test tube, the mixture of DDQ (136.2 mg, $0.6 \mathrm{mmol})$, AgOTf $(7.8 \mathrm{mg}, 0.03 \mathrm{mmol})$ and $\mathrm{PhCl}(3.0 \mathrm{~mL})$ was added $10^{9}(73.0 \mathrm{mg}, 0.3 \mathrm{mmol})$ and ${ }^{t} \mathrm{BuNC}(136 \mu \mathrm{L}, 1.2 \mathrm{mmol})$. The mixture was stirred at $80{ }^{\circ} \mathrm{C}$ for about $3 \mathrm{hrs}$ under $\mathrm{N}_{2}$ as monitored by TLC. Upon completion, the reaction mixture was cooled 
down to room temperature and concentrated under vacuum. The residue was purified by column chromatography on silica gel (petroleum ether/ethyl acetate $=100: 1, \mathrm{v} / \mathrm{v}$ ) to give $\mathbf{2 0}$ as a light yellow solid (41.1 mg, 47\% yield). m.p.: $38-39^{\circ} \mathrm{C}$; IR $\left(\mathrm{KBr}, \mathrm{cm}^{-1}\right)$ : 3065, 2977, 2930, 2864, 2217 , 1698, 1621, 1292, 919, 752; ${ }^{1} \mathrm{H}$ NMR ( $\left.\mathrm{CDCl}_{3}, 500 \mathrm{MHz}\right): \delta 7.58$ (d, $\left.J=7.8 \mathrm{~Hz}, 1 \mathrm{H}\right), 7.41(\mathrm{~d}, J=$ $4.4 \mathrm{~Hz}, 1 \mathrm{H}), 7.39-7.35$ (m, 2H), 1.44 (s, 9H); ${ }^{13} \mathrm{C} \mathrm{NMR}\left(\mathrm{CDCl}_{3}, 125 \mathrm{MHz}\right): \delta 190.2,137.6,136.5$, 133.1, 132.7, 130.7, 127.4, 120.3, 110.4, 60.8, 28.8; EI-MS $m / z$ (\%): $294.0(1)\left[\mathrm{M}\left({ }^{81} \mathrm{Br}\right)\right]^{+}, 292.0$ (1) $\left[\mathrm{M}\left({ }^{79} \mathrm{Br}\right)\right]^{+}, 279.0$ (100), 277.0 (98), 260.2 (25); HRMS (EI) $m / z$ calcd for $\mathrm{C}_{13} \mathrm{H}_{13} \mathrm{~N}_{2} \mathrm{OBr}[\mathrm{M}]^{+}$ 292.0211, found 292.0219.<smiles></smiles>

(E)-N-(tert-Butyl)-2-oxo-2-(m-tolyl)acetimidoyl cyanide (2p): To a test tube, the mixture of DDQ (136.2 mg, $0.6 \mathrm{mmol})$, AgOTf (7.8 mg, $0.03 \mathrm{mmol})$ and $\mathrm{PhCl}(3.0 \mathrm{~mL})$ was added $1 \mathbf{p}^{2}(53.5$ $\mathrm{mg}, 0.3 \mathrm{mmol})$ and ${ }^{t} \mathrm{BuNC}(136 \mu \mathrm{L}, 1.2 \mathrm{mmol})$. The mixture was stirred at $80{ }^{\circ} \mathrm{C}$ for about $3 \mathrm{hrs}$ under $\mathrm{N}_{2}$ as monitored by TLC. Upon completion, the reaction mixture was cooled down to room temperature and concentrated under vacuum. The residue was purified by column chromatography on silica gel (petroleum ether/ethyl acetate $=100: 1, \mathrm{v} / \mathrm{v})$ to give $\mathbf{2 p}$ as a yellow oil $(57.2 \mathrm{mg}, 83 \%$ yield). IR ( $\left.\mathrm{KBr}, \mathrm{cm}^{-1}\right): 2977,2217,1674,1594,1290,1231,946,795,754 ;{ }^{1} \mathrm{H}$ NMR $\left(\mathrm{CDCl}_{3}, 500\right.$ MHz): $\delta 7.85-7.82(\mathrm{~m}, 2 \mathrm{H}), 7.44(\mathrm{~d}, J=8.5 \mathrm{~Hz}, 1 \mathrm{H}), 7.35(\mathrm{t}, J=7.6 \mathrm{~Hz}, 1 \mathrm{H}), 2.41(\mathrm{~s}, 3 \mathrm{H}), 1.55(\mathrm{~s}$,

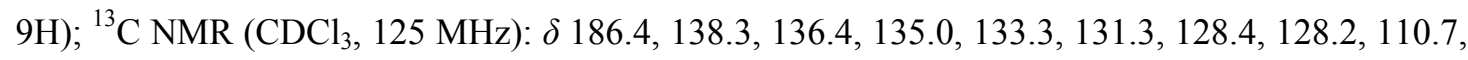
60.7, 29.0, 21.4; EI-MS m/z (\%): 228 (0.5) [M] ${ }^{+}, 119$ (100), 91 (34); HRMS (EI) $m / z$ calcd for $\mathrm{C}_{14} \mathrm{H}_{16} \mathrm{~N}_{2} \mathrm{O}[\mathrm{M}]^{+} 228.1263$, found 228.1265 .

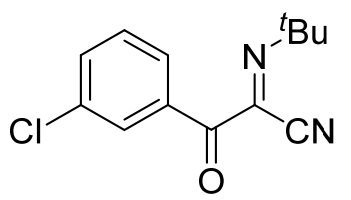

(E)-N-(tert-Butyl)-2-(3-chlorophenyl)-2-oxoacetimidoyl cyanide (2q): To a test tube, the mixture of DDQ (136.2 mg, $0.6 \mathrm{mmol})$, AgOTf $(7.8 \mathrm{mg}, 0.03 \mathrm{mmol})$ and $\mathrm{PhCl}(3.0 \mathrm{~mL})$ was added $1 \mathbf{q}^{5}$ (59.6 $\left.\mathrm{mg}, 0.3 \mathrm{mmol}\right)$ and ${ }^{t} \mathrm{BuNC}(136 \mu \mathrm{L}, 1.2 \mathrm{mmol})$. The mixture was stirred at $80{ }^{\circ} \mathrm{C}$ for about $3 \mathrm{hrs}$ under $\mathrm{N}_{2}$ as monitored by TLC. Upon completion, the reaction mixture was cooled down to room temperature and concentrated under vacuum. The residue was purified by column chromatography on silica gel (petroleum ether/ethyl acetate $=100: 1, \mathrm{v} / \mathrm{v}$ ) to give $\mathbf{2 q}$ as a yellow oil (52.8 mg, 70\% yield). IR (KBr, cm $\left.{ }^{-1}\right): 3736,2973,2214,1687,1524,1371,1225,1047,915$, 868, 751; ${ }^{1} \mathrm{H} \mathrm{NMR}\left(\mathrm{CDCl}_{3}, 500 \mathrm{MHz}\right): \delta 8.05(\mathrm{~s}, 1 \mathrm{H}), 7.93(\mathrm{~d}, J=8.1 \mathrm{~Hz}, 1 \mathrm{H}), 7.59$ (d, $J=7.7$ $\mathrm{Hz}, 1 \mathrm{H}), 7.28$ (t, $J=7.9 \mathrm{~Hz}, 1 \mathrm{H}), 1.55(\mathrm{~s}, 9 \mathrm{H}) ;{ }^{13} \mathrm{C} \mathrm{NMR}\left(\mathrm{CDCl}_{3}, 125 \mathrm{MHz}\right): \delta 184.9,136.2$, 134.9, 134.6, 134.0, 131.0, 129.7, 129.1, 110.3, 61.0, 29.0; EI-MS m/z (\%): 248.1 (1) [M] ${ }^{+}, 236.1$ (10), 235.0 (50), 234.1 (20), 233.0 (100), 230.1 (10); HRMS (EI) $\mathrm{m} / z$ calcd for $\mathrm{C}_{13} \mathrm{H}_{13} \mathrm{~N}_{2} \mathrm{OCl}[\mathrm{M}]^{+}$ 248.0716, found 248.0723.<smiles>N#CC(=NC(=O)c1cccc(Br)c1)C(=O)c1ccccc1</smiles>

(E)-2-(3-Bromophenyl)- $N$-(tert-butyl)-2-oxoacetimidoyl cyanide (2r): To a test tube, the 
mixture of DDQ (136.2 mg, $0.6 \mathrm{mmol}), \operatorname{AgOTf}(7.8 \mathrm{mg}, 0.03 \mathrm{mmol})$ and $\mathrm{PhCl}(3.0 \mathrm{~mL})$ was added $1 \mathbf{r}(73.0 \mathrm{mg}, 0.3 \mathrm{mmol})$ and ${ }^{t} \mathrm{BuNC}(136 \mu \mathrm{L}, 1.2 \mathrm{mmol})$. The mixture was stirred at $80{ }^{\circ} \mathrm{C}$ for about $3 \mathrm{hrs}$ under $\mathrm{N}_{2}$ as monitored by TLC. Upon completion, the reaction mixture was cooled down to room temperature and concentrated under vacuum. The residue was purified by column chromatography on silica gel (petroleum ether/ethyl acetate $=100: 1, \mathrm{v} / \mathrm{v}$ ) to give $\mathbf{2 r}$ as a yellow oil (61.5 mg, 70\% yield). IR (KBr, cm $\left.{ }^{-1}\right): 2967,2925,2221,1680,1466,1230,927,758,717 ;{ }^{1} \mathrm{H}$ NMR ( $\left.\mathrm{CDCl}_{3}, 500 \mathrm{MHz}\right): \delta 8.21(\mathrm{t}, J=1.8 \mathrm{~Hz}, 1 \mathrm{H}), 7.99-7.97(\mathrm{~m}, 1 \mathrm{H}), 7.75-7.73(\mathrm{~m}, 1 \mathrm{H}), 7.35(\mathrm{t}$, $J=7.9 \mathrm{~Hz}, 1 \mathrm{H}), 1.55(\mathrm{~s}, 9 \mathrm{H}) ;{ }^{13} \mathrm{C} \mathrm{NMR}\left(\mathrm{CDCl}_{3}, 125 \mathrm{MHz}\right): \delta 184.9,137.0,136.3,135.2,134.1$, 130.0, 129.7, 122.6, 110.4, 61.2, 29.1; EI-MS m/z (\%): 294.0 (1) [M $\left.\left({ }^{81} \mathrm{Br}\right)\right]^{+}, 292.0(1)\left[\mathrm{M}\left({ }^{79} \mathrm{Br}\right)\right]^{+}$, 184.9 (99), 182.9 (100), 156.9 (50), 154.9 (50); HRMS (EI) $m / z$ calcd for $\mathrm{C}_{13} \mathrm{H}_{13} \mathrm{~N}_{2} \mathrm{OBr}[\mathrm{M}]^{+}$ 292.0211, found 292.0213 .<smiles>CCCCN=C(C#N)C(=O)c1cccc(OC)c1</smiles>

(E)-N-(tert-Butyl)-2-(3-methoxyphenyl)-2-oxoacetimidoyl cyanide (2s): To a test tube, the mixture of DDQ (136.2 mg, $0.6 \mathrm{mmol})$, AgOTf $(7.8 \mathrm{mg}, 0.03 \mathrm{mmol})$ and $\mathrm{PhCl}(3.0 \mathrm{~mL})$ was added $1 \mathbf{s}^{10}(58.3 \mathrm{mg}, 0.3 \mathrm{mmol})$ and ${ }^{t} \mathrm{BuNC}(136 \mu \mathrm{L}, 1.2 \mathrm{mmol})$. The mixture was stirred at $80{ }^{\circ} \mathrm{C}$ for about $3 \mathrm{hrs}$ under $\mathrm{N}_{2}$ as monitored by TLC. Upon completion, the reaction mixture was cooled down to room temperature and concentrated under vacuum. The residue was purified by column chromatography on silica gel (petroleum ether/ethyl acetate $=100: 1, \mathrm{v} / \mathrm{v}$ ) to give $2 \mathbf{s}$ as a yellow oil (51.5 mg, 70\% yield). IR (KBr, cm ${ }^{-1}$ ): 3842, 3743, 2974, 2221, 1675, 1589, 1474, 1289, 1042, 796, 680; ${ }^{1} \mathrm{H} \mathrm{NMR}\left(\mathrm{CDCl}_{3}, 500 \mathrm{MHz}\right): \delta 7.63(\mathrm{~d}, J=7.6 \mathrm{~Hz}, 1 \mathrm{H}), 7.60(\mathrm{~s}, 1 \mathrm{H}), 7.38(\mathrm{t}, J=7.9 \mathrm{~Hz}, 1 \mathrm{H})$,

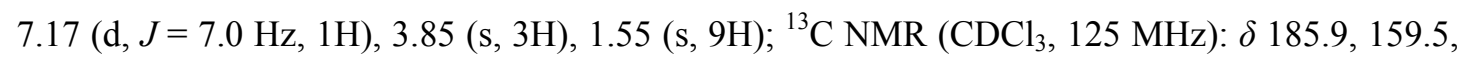
136.4, 134.4, 129.4, 123.9, 121.0, 114.9, 110.6, 60.8, 55.5, 29.0; EI-MS $m / z$ (\%): 244 (2) [M] ${ }^{+}$, 152 (9), 135 (100), 107 (18), 92 (11), 77 (15), 57 (12); HRMS (EI) $m / z$ calcd for $\mathrm{C}_{14} \mathrm{H}_{16} \mathrm{~N}_{2} \mathrm{O}_{2}[\mathrm{M}]^{+}$ 244.1212, found 244.1210 .

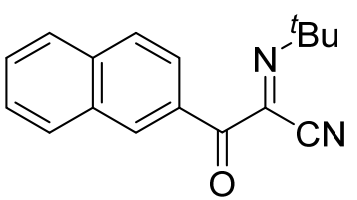

(E)-N-(tert-Butyl)-2-(naphthalen-2-yl)-2-oxoacetimidoyl cyanide (2t): To a test tube, the mixture of DDQ (136.2 mg, $0.6 \mathrm{mmol})$, AgOTf $(7.8 \mathrm{mg}, 0.03 \mathrm{mmol})$ and $\mathrm{PhCl}(3.0 \mathrm{~mL})$ was added $\mathbf{1 t}^{11}$ (64.3 mg, $\left.0.3 \mathrm{mmol}\right)$ and ${ }^{t} \mathrm{BuNC}(136 \mu \mathrm{L}, 1.2 \mathrm{mmol})$. The mixture was stirred at $80{ }^{\circ} \mathrm{C}$ for about $3 \mathrm{hrs}$ under $\mathrm{N}_{2}$ as monitored by TLC. Upon completion, the reaction mixture was cooled down to room temperature and concentrated under vacuum. The residue was purified by column chromatography on silica gel (petroleum ether/ethyl acetate $=100: 1, \mathrm{v} / \mathrm{v}$ ) to give $\mathbf{2 t}$ as a light yellow solid (39.3 mg, 50\% yield). m.p.: $65-66{ }^{\circ} \mathrm{C}$; IR $\left(\mathrm{KBr}, \mathrm{cm}^{-1}\right): 3742,3679,3435,2974,2217$, 2159, 1661, 1622, 1509, 1464, 1360, 1198, 1092, 966, 924, 768; ${ }^{1} \mathrm{H}$ NMR $\left(\mathrm{CDCl}_{3}, 500 \mathrm{MHz}\right): \delta$ $8.66(\mathrm{~s}, 1 \mathrm{H}), 8.07-8.05(\mathrm{~m}, 1 \mathrm{H}), 7.95(\mathrm{~d}, J=8.1 \mathrm{~Hz}, 1 \mathrm{H}), 7.90(\mathrm{t}, J=8.4 \mathrm{~Hz}, 2 \mathrm{H}), 7.66-7.63(\mathrm{~m}$, 1H), 7.59-7.56 (m, 1H), $1.60(\mathrm{~s}, 9 \mathrm{H}) ;{ }^{13} \mathrm{C} \mathrm{NMR}\left(\mathrm{CDCl}_{3}, 125 \mathrm{MHz}\right): \delta 186.0,136.6,135.9,134.0$, 132.1, 130.6, 130.0, 129.3, 128.3, 127.8, 127.0, 125.5, 110.7, 60.9, 29.1; LC-MS (ESI) $\mathrm{m} / z 265.1$ $[\mathrm{M}+\mathrm{H}]^{+}$; HRMS (DART) $\mathrm{m} / z$ calcd for $\mathrm{C}_{17} \mathrm{H}_{17} \mathrm{~N}_{2} \mathrm{O}[\mathrm{M}+\mathrm{H}]^{+}$265.1335, found 265.1334. 
<smiles>CC(C)/N=C(\C#N)C(=O)c1ccsc1</smiles>

(E)-N-(tert-Butyl)-2-oxo-2-(thiophen-3-yl)acetimidoyl cyanide (2u): To a test tube, the mixture of DDQ (136.2 mg, $0.6 \mathrm{mmol})$, AgOTf $(7.8 \mathrm{mg}, 0.03 \mathrm{mmol})$ and $\mathrm{PhCl}(3.0 \mathrm{~mL})$ was added $\mathbf{1 u}$ $(51.1 \mathrm{mg}, 0.3 \mathrm{mmol})$ and ${ }^{t} \mathrm{BuNC}(136 \mu \mathrm{L}, 1.2 \mathrm{mmol})$. The mixture was stirred at $80{ }^{\circ} \mathrm{C}$ for about 3 hrs under $\mathrm{N}_{2}$ as monitored by TLC. Upon completion, the reaction mixture was cooled down to room temperature and concentrated under vacuum. The residue was purified by column chromatography on silica gel (petroleum ether/ethyl acetate $=100: 1, \mathrm{v} / \mathrm{v}$ ) to give $\mathbf{2} \mathbf{u}$ as a yellow solid (42.9 mg, 65\% yield). m.p.: $44-45^{\circ} \mathrm{C}$; IR $\left(\mathrm{KBr}, \mathrm{cm}^{-1}\right)$ : 3740, 3129, 2973, 2225, 1654, 1511, $1423,1275,1110,952,832,761 ;{ }^{1} \mathrm{H}$ NMR $\left(\mathrm{CDCl}_{3}, 500 \mathrm{MHz}\right): \delta 8.63(\mathrm{dd}, J=4.1,1.2 \mathrm{~Hz}, 1 \mathrm{H})$, $7.75(\mathrm{dd}, J=6.3,1.2 \mathrm{~Hz}, 1 \mathrm{H}), 7.33(\mathrm{dd}, J=5.3,3.1 \mathrm{~Hz}, 1 \mathrm{H}), 1.57$ (s, 9H); ${ }^{13} \mathrm{C} \mathrm{NMR}\left(\mathrm{CDCl}_{3}, 125\right.$ MHz): $\delta 179.1,137.7,137.6,137.0,129.1,125.9,110.5,60.7,29.1$; EI-MS $m / z$ (\%): 220.1 (10) $[\mathrm{M}]^{+}, 111.7$ (70), 111.0 (100), 83.0 (50), 57.1 (50); HRMS (EI) $\mathrm{m} / z$ calcd for $\mathrm{C}_{11} \mathrm{H}_{12} \mathrm{~N}_{2} \mathrm{OS}[\mathrm{M}]^{+}$ 220.0670 , found 220.0665 .<smiles>CCCCN=C(C#N)C(=O)c1ccc(-c2ccc(C(=O)/C(C#N)=N/C(C)C)cc2)cc1</smiles>

(1E,1'E)-2,2'-([1,1'-Biphenyl]-4,4'-diyl)bis( $N$-(tert-butyl)-2-oxoacetimidoyl cyanide) (2v): To a test tube, the mixture of DDQ (272.4 mg, $1.2 \mathrm{mmol})$, AgOTf (15.6 mg, $0.06 \mathrm{mmol})$ and $\mathrm{PhCl}(3.0$ $\mathrm{mL}$ ) was added $1 \mathbf{v}(98.0 \mathrm{mg}, 0.3 \mathrm{mmol})$ and ${ }^{t} \mathrm{BuNC}(272 \mu \mathrm{L}, 2.4 \mathrm{mmol})$. The mixture was stirred at $80{ }^{\circ} \mathrm{C}$ for about $3 \mathrm{hrs}$ under $\mathrm{N}_{2}$ as monitored by TLC. Upon completion, the reaction mixture was cooled down to room temperature and concentrated under vacuum. The residue was purified by column chromatography on silica gel (from petroleum ether/ethyl acetate $=50: 1$ to petroleum ether/ethyl acetate $=20: 1, \mathrm{v} / \mathrm{v})$ to give $2 \mathbf{v}$ as a yellow solid $(104.4 \mathrm{mg}, 81 \%$ yield). m.p.: 127-128 ${ }^{\circ} \mathrm{C}$; IR (KBr, cm $\left.{ }^{-1}\right): 3740,2979,2219,1924,1669,1603,1464,1286,1120,917,867,764,617$; ${ }^{1} \mathrm{H}$ NMR $\left(\mathrm{CDCl}_{3}, 500 \mathrm{MHz}\right): \delta 8.18(\mathrm{~d}, J=8.4 \mathrm{~Hz}, 4 \mathrm{H}), 7.74(\mathrm{~d}, J=8.5 \mathrm{~Hz}, 4 \mathrm{H}), 1.58(\mathrm{~s}, 18 \mathrm{H})$; ${ }^{13} \mathrm{C} \mathrm{NMR}\left(\mathrm{CDCl}_{3}, 125 \mathrm{MHz}\right): \delta 185.7,145.1,136.7,133.2,131.9,127.4,110.7,61.0,29.2$; LC-MS (ESI) $m / z 427.2[\mathrm{M}+\mathrm{H}]^{+}$; HRMS (DART) $m / z$ calcd for $\mathrm{C}_{26} \mathrm{H}_{27} \mathrm{~N}_{4} \mathrm{O}_{2}[\mathrm{M}+\mathrm{H}]^{+} 427.2129$, found 427.2130 .

\section{Synthetic Applications of $\beta$-Carbonyl $\alpha$-Iminonitrile 2a}<smiles>CC(C)C=NC(C#N)C(=O)c1ccccc1</smiles>

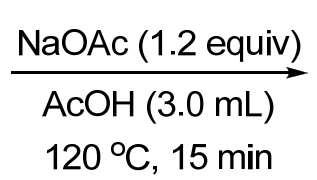

$120^{\circ} \mathrm{C}, 15 \mathrm{~min}$<smiles>N#Cc1nc2ccccc2nc1-c1ccccc1</smiles>

3

3-phenylquinoxaline-2-carbonitrile (3): To a test tube was added 2a $(64.3 \mathrm{mg}, 0.30 \mathrm{mmol})$, 1,2-diaminobenzene (15.6 mg, $0.36 \mathrm{mmol}), \mathrm{NaOAc}(29.5 \mathrm{mg}, 0.36 \mathrm{mmol})$, and $\mathrm{AcOH}(3.0 \mathrm{~mL})$. 
The resulting mixture was stirred at $120{ }^{\circ} \mathrm{C}$ for 15 min under air as monitored by TLC. Upon completion, the reaction mixture was cooled down to room temperature and extracted with ethyl acetate. The organic layer was then washed with $\mathrm{H}_{2} \mathrm{O}$ and brine, dried over anhydrous $\mathrm{Na}_{2} \mathrm{SO}_{4}$, filtered and concentrated in vacuo. The resulting residue was purified by column chromatography on silica gel (petroleum ether/ethyl acetate $=50: 1, \mathrm{v} / \mathrm{v}$ ) to give the desired product 3 as a white solid $\left(65.7 \mathrm{mg}\right.$, 95\% yield). m.p.: $164-165{ }^{\circ} \mathrm{C}$; IR $\left(\mathrm{KBr}, \mathrm{cm}^{-1}\right): 3989,3543,3474,3414,3142$, 1626, 1400, 1076, 617; ${ }^{1} \mathrm{H}$ NMR ( $\left.\mathrm{CDCl}_{3}, 500 \mathrm{MHz}\right): \delta 8.21-8.18$ (m, 2H), 8.06-8.04 (m, 2H), 7.94 $(\mathrm{t}, J=7.7 \mathrm{~Hz}, 1 \mathrm{H}), 7.88(\mathrm{t}, J=7.3 \mathrm{~Hz}, 1 \mathrm{H}), 7.61-7.60(\mathrm{~m}, 3 \mathrm{H}) ;{ }^{13} \mathrm{C} \mathrm{NMR}\left(\mathrm{CDCl}_{3}, 125 \mathrm{MHz}\right): \delta$ 154.4, 142.5, 140.7, 135.2, 133.7, 131.5, 130.9, 129.7, 129.6, 129.4, 129.1, 128.5, 116.7; LC-MS (ESI) $m / z 232.1[\mathrm{M}+\mathrm{H}]^{+}$; HRMS (DART) $m / z$ calcd for $\mathrm{C}_{15} \mathrm{H}_{10} \mathrm{~N}_{3}[\mathrm{M}+\mathrm{H}]^{+} 232.0869$, found 232.0868 .<smiles>N#Cc1nc2ccccc2nc1-c1ccccc1</smiles>

3<smiles>CO[n+]1c(C#N)c(-c2ccccc2)[n+]([O-])c2ccccc21</smiles>

2-Cyano-3-phenylquinoxaline 1,4-dioxide (4): To a test tube was added 3 (69.4 mg, $0.30 \mathrm{mmol})$, $85 \% \mathrm{~m}$-CPBA (365.4 mg, $1.80 \mathrm{mmol})$ and DCE $(2.0 \mathrm{~mL})$. The resulting mixture was stirred at 80 ${ }^{\circ} \mathrm{C}$ for $48 \mathrm{~h}$ under air as monitored by TLC. Upon completion, the reaction mixture was cooled down to room temperature and was quenched with $10 \% \mathrm{Na}_{2} \mathrm{SO}_{3}$ overnight. The mixture was extracted with ethyl acetate. The organic layer was then washed with $\mathrm{H}_{2} \mathrm{O}$ and brine, dried over anhydrous $\mathrm{Na}_{2} \mathrm{SO}_{4}$, filtered and concentrated in vacuo. The residue was purified by column chromatography on silica gel (from petroleum ether/ethyl acetate $=20: 1$ to petroleum ether/ethyl acetate $=7: 1, \mathrm{v} / \mathrm{v})$ to give the desired product 4 as a yellow solid $(24.5 \mathrm{mg}, 31 \%$ yield $)$. m.p.: 204-205 ${ }^{\circ} \mathrm{C}$; IR $\left(\mathrm{KBr}, \mathrm{cm}^{-1}\right): 3995,3391,3125,2062,1626,1400,1072,615 ;{ }^{1} \mathrm{H}$ NMR $\left(\mathrm{CDCl}_{3}\right.$, $500 \mathrm{MHz}): \delta 8.63-8.61(\mathrm{~m}, 1 \mathrm{H}), 8.23-8.21(\mathrm{~m}, 1 \mathrm{H}), 7.95-7.89(\mathrm{~m}, 2 \mathrm{H}), 7.73-7.71(\mathrm{~m}, 2 \mathrm{H})$,

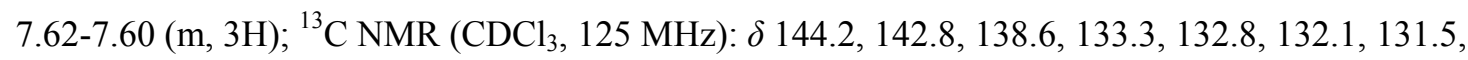
131.0, 130.2, 129.1, 127.3, 119.6, 115.0; LC-MS (ESI) $m / z 264[\mathrm{M}+\mathrm{H}]^{+}$; HRMS (DART) $\mathrm{m} / \mathrm{z}$ calcd for $\mathrm{C}_{15} \mathrm{H}_{10} \mathrm{~N}_{3} \mathrm{O}_{2}[\mathrm{M}+\mathrm{H}]^{+}$264.0768, found 264.0767.

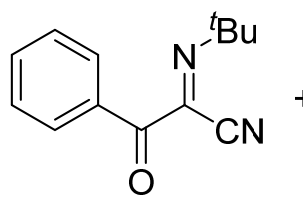

2a<smiles>Nc1ccccc1O</smiles><smiles>NC(=O)CCCCCC(=O)OCC(=O)O</smiles>
$120^{\circ} \mathrm{C}$, air

Benzo[d] oxazol-2-yl(phenyl)methanone (5a) ${ }^{12}$ : To a test tube was added 2a $(64.3 \mathrm{mg}, 0.30$ mmol), 2-aminophenol (39.3 mg, $0.36 \mathrm{mmol}), \mathrm{NaOAc}(29.5 \mathrm{mg}, 0.36 \mathrm{mmol})$, and $\mathrm{AcOH}(3.0 \mathrm{~mL})$. The resulting mixture was stirred at $120{ }^{\circ} \mathrm{C}$ for $4 \mathrm{~h}$ under air as monitored by TLC. Upon completion, the reaction mixture was cooled down to room temperature and extracted with ethyl acetate. The organic layer was then washed with $\mathrm{H}_{2} \mathrm{O}$ and brine, dried over anhydrous $\mathrm{Na}_{2} \mathrm{SO}_{4}$, filtered and concentrated in vacuo. The residue was purified by column chromatography on silica gel (petroleum ether/ethyl acetate $=30: 1, \mathrm{v} / \mathrm{v}$ ) to give the desired product $\mathbf{5 a}$ as a white solid (53.7 mg, 80\% yield). m.p.: $61-62{ }^{\circ} \mathrm{C}$; IR ( $\left.\mathrm{KBr}, \mathrm{cm}^{-1}\right): 3082,2359,2064,1622,1402,1389,1070,874$, 
779, 615; ${ }^{1} \mathrm{H}$ NMR $\left(\mathrm{CDCl}_{3}, 500 \mathrm{MHz}\right): \delta 8.56-8.54(\mathrm{~m}, 2 \mathrm{H}), 7.96(\mathrm{~d}, J=8.0 \mathrm{~Hz}, 1 \mathrm{H}), 7.73-7.68$ (m, 2H), 7.59-7.54 (m, 3H), $7.48(\mathrm{t}, J=7.8 \mathrm{~Hz}, 1 \mathrm{H}) ;{ }^{13} \mathrm{C} \mathrm{NMR}\left(\mathrm{CDCl}_{3}, 125 \mathrm{MHz}\right): \delta 180.7,157.3$, 150.6, 140.9, 135.2, 134.5, 131.2, 128.8, 128.6, 125.9, 122.6, 112.0; EI-MS $m / z(\%): 223.1$ (30) $[\mathrm{M}]^{+}, 195.1(40), 105.0(100), 77.0(40)$.

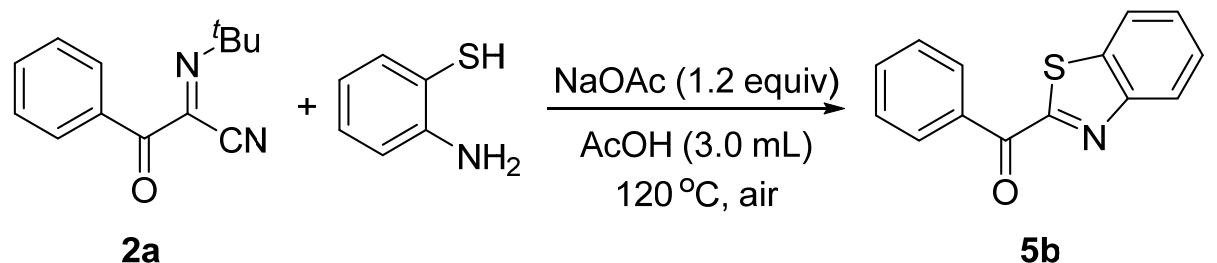

Benzo[d] thiazol-2-yl(phenyl)methanone (5b) ${ }^{12}$ : To a test tube was added 2a $(64.3 \mathrm{mg}, 0.30$ mmol), 2-aminobenzenethiol (45.1 mg, $0.36 \mathrm{mmol}), \mathrm{NaOAc}(29.5 \mathrm{mg}, 0.36 \mathrm{mmol})$, and $\mathrm{AcOH}$ $(3.0 \mathrm{~mL})$. The resulting mixture was stirred at $120{ }^{\circ} \mathrm{C}$ for $4 \mathrm{~h}$ under air as monitored by TLC. Upon completion, the reaction mixture was cooled down to room temperature and extracted with ethyl acetate. The organic layer was then washed with $\mathrm{H}_{2} \mathrm{O}$ and brine, dried over anhydrous $\mathrm{Na}_{2} \mathrm{SO}_{4}$, filtered and concentrated in vacuo. The residue was purified by column chromatography on silica gel (petroleum ether/ethyl acetate $=30: 1, \mathrm{v} / \mathrm{v}$ ) to give the desired product $\mathbf{5 b}$ as a white solid (41.7 mg, 58\% yield). m.p.: 95-96 ${ }^{\circ} \mathrm{C} ;{ }^{1} \mathrm{H}$ NMR $\left(\mathrm{CDCl}_{3}, 500 \mathrm{MHz}\right): \delta 8.56(\mathrm{~d}, J=7.4 \mathrm{~Hz}$, $2 \mathrm{H}), 8.25(\mathrm{~d}, J=7.8 \mathrm{~Hz}, 1 \mathrm{H}), 8.02(\mathrm{~d}, J=7.6 \mathrm{~Hz}, 1 \mathrm{H}), 7.68(\mathrm{t}, J=7.4 \mathrm{~Hz}, 1 \mathrm{H}), 7.61-7.54(\mathrm{~m}, 4 \mathrm{H})$; ${ }^{13} \mathrm{C} \mathrm{NMR}\left(\mathrm{CDCl}_{3}, 125 \mathrm{MHz}\right): \delta 185.5,167.3,154.0,137.2,135.1,134.0,131.4,128.6,127.8$, 127.1, 125.9, 122.3; LC-MS (ESI) $\mathrm{m} / z 262[\mathrm{M}+\mathrm{H}]^{+}$.

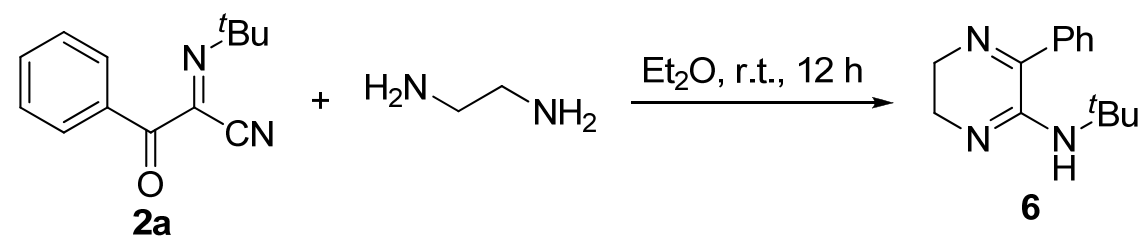

$\mathrm{N}$-(tert-Butyl)-3-phenyl-5,6-dihydropyrazin-2-amine (6): To a test tube was added 2a (64.3 $\mathrm{mg}$, $0.30 \mathrm{mmol})$, ethylenediamine $(21.6 \mathrm{mg}, 0.36 \mathrm{mmol})$, and $\mathrm{Et}_{2} \mathrm{O}(3.0 \mathrm{~mL})$. The resulting mixture was stirred at room temperature for $12 \mathrm{~h}$ under air as monitored by TLC. Upon completion, the reaction mixture was extracted with ethyl acetate. The organic layer was then washed with $\mathrm{H}_{2} \mathrm{O}$ and brine, dried over anhydrous $\mathrm{Na}_{2} \mathrm{SO}_{4}$, filtered and concentrated in vacuo. The residue was purified by column chromatography on silica gel (from petroleum ether/ethyl acetate $=10: 1$ to petroleum ether/ethyl acetate $=1: 1, \mathrm{v} / \mathrm{v})$ to give the desired product $\mathbf{6}$ as a white solid $(67.5 \mathrm{mg}, 98 \%$ yield). m.p.: $134-135{ }^{\circ} \mathrm{C}$; IR $\left(\mathrm{KBr}, \mathrm{cm}^{-1}\right): 3127,2833,2054,1626,1396,1219,1072,617 ;{ }^{1} \mathrm{H}$ NMR (CDCl 3 , $500 \mathrm{MHz}): \delta$ 7.58-7.56 (m, 2H), 7.44-7.40 (m, 3H), $4.06(\mathrm{br}, 1 \mathrm{H}), 3.57-3.54(\mathrm{~m}$, 2H), 3.37-3.34 (m, 2H), 1.35 (s, 9H); ${ }^{13} \mathrm{C}$ NMR ( $\left.\mathrm{CDCl}_{3}, 125 \mathrm{MHz}\right): \delta 160.3,147.9,136.5,130.2$, 128.7, 128.4, 51.5, 47.5, 43.4, 28.8; LC-MS (ESI) $\mathrm{m} / z 230.2[\mathrm{M}+\mathrm{H}]^{+}$; HRMS (DART) $\mathrm{m} / z$ calcd for $\mathrm{C}_{14} \mathrm{H}_{20} \mathrm{~N}_{3}[\mathrm{M}+\mathrm{H}]^{+} 230.1652$, found 230.1651 .<smiles>[14CH3]/N=C(\C#N)C(=O)c1ccccc1</smiles>

$2 a$<smiles>CCOCCNC1=NC2CCCCC2N=C1c1ccccc1</smiles>

(cis)- $N$-(tert-Butyl)-3-phenyl-4a,5,6,7,8,8a-hexahydroquinoxalin-2-amine (7): To a test tube 
was added 2a $(64.3 \mathrm{mg}, 0.30 \mathrm{mmol})$, cis-cyclohexane-1,2-diamine (41.1 $\mathrm{mg}, 0.36 \mathrm{mmol})$, and $\mathrm{Et}_{2} \mathrm{O}(3.0 \mathrm{~mL})$. The resulting mixture was stirred at room temperature for $5 \mathrm{~h}$ under air as monitored by TLC. Upon completion, the reaction mixture was extracted with ethyl acetate. The organic layer was then washed with $\mathrm{H}_{2} \mathrm{O}$ and brine, dried over anhydrous $\mathrm{Na}_{2} \mathrm{SO}_{4}$, filtered and concentrated in vacuo. The residue was purified by column chromatography on silica gel (from petroleum ether/ethyl acetate $=10: 1$ to petroleum ether/ethyl acetate $=1: 1, \mathrm{v} / \mathrm{v}$ ) to give the desired product 7 as a yellow oil $\left(81.7 \mathrm{mg}, 96 \%\right.$ yield). IR (KBr, $\left.\mathrm{cm}^{-1}\right): 3069,2619,2039,1632,1501$, 1406, 1223, 1074, 611, 503; ${ }^{1} \mathrm{H}$ NMR $\left(\mathrm{CDCl}_{3}, 500 \mathrm{MHz}\right): \delta$ 7.56-7.54 (m, 2H), 7.43-7.39 (m, 3H), 3.97 (br, 1H), 3.69-3.66 (m, 1H), 3.49-3.46 (m, 1H), 1.75-1.49 (m, 6H), $1.41(\mathrm{~d}, J=8.1 \mathrm{~Hz}, 1 \mathrm{H})$,

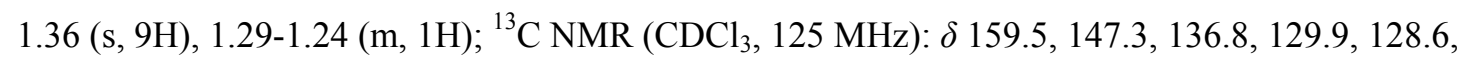

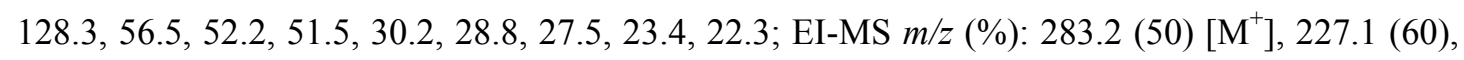
226.1 (100), 96.1 (20); HRMS (EI) $m / z$ calcd for $\mathrm{C}_{18} \mathrm{H}_{25} \mathrm{~N}_{3}[\mathrm{M}]^{+}$283.2048, found 283.2047.<smiles>CC(C)/N=C(\C#N)C(=O)c1ccccc1</smiles>

$2 \mathbf{a}$

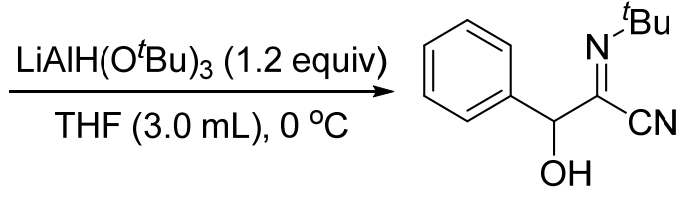

8

(E)-N-(tert-Butyl)-2-hydroxy-2-phenylacetimidoyl cyanide (8): To a test tube was added 2a (64.3 $\mathrm{mg}, 0.30 \mathrm{mmol})$ and THF $(3.0 \mathrm{~mL})$, followed by the addition of $\mathrm{LiAlH}\left(\mathrm{O}^{t} \mathrm{Bu}\right)_{3}(1 \mathrm{M}$ in THF, $0.36 \mathrm{~mL}, 0.36 \mathrm{mmol})$. The mixture was then stirred at $0{ }^{\circ} \mathrm{C}$ for $10 \mathrm{~min}$ until the reaction was completed (monitored by TLC). Upon completion, the reaction was carefully quenched with saturated potassium sodium tartrate $(10 \mathrm{~mL})$. The reaction mixture was extracted with ethyl acetate. The organic layer was then washed with $\mathrm{H}_{2} \mathrm{O}$ and brine, dried over anhydrous $\mathrm{Na}_{2} \mathrm{SO}_{4}$, filtered and concentrated in vacuo. The residue was purified by fast column chromatography on silica gel (petroleum ether/ethyl acetate $=15: 1, \mathrm{v} / \mathrm{v}$ ) to give the desired product 8 as a white solid (61.8 mg, 95\% yield). m.p.: 38-39 ${ }^{\circ} \mathrm{C}$; IR ( $\left.\mathrm{KBr}, \mathrm{cm}^{-1}\right)$ : 3288, 2975, 1638, 1376, 1196, 1051, 698; ${ }^{1}$ H NMR (Acetone- $\left.d_{6}, 500 \mathrm{MHz}\right): \delta$ 7.48-7.46 (m, 2H), 7.43-7.40 (m, 2H), 7.37-7.34 (m, 1H), 5.38 (s, 1H), 1.40 (s, 9H); ${ }^{13} \mathrm{C}$ NMR (Acetone- $d_{6}, 125 \mathrm{MHz}$ ): $\delta 143.2,139.5,129.4,129.2,127.5,111.9$, 78.1, 58.7, 29.3; LC-MS $m / z 217[\mathrm{M}+\mathrm{H}]^{+}$; HRMS $m / z$ calcd for $\mathrm{C}_{13} \mathrm{H}_{17} \mathrm{ON}_{2}[\mathrm{M}+\mathrm{H}]^{+} 217.1335$, found 217.1334 .

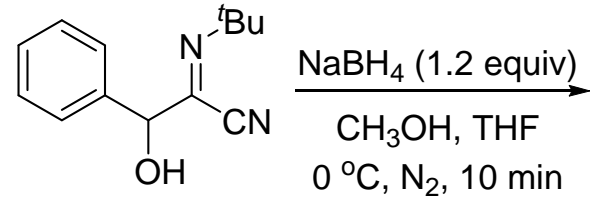

8<smiles>CC(C)CNC(C#N)[C@H](O)c1ccccc1</smiles>

9

trans-2-(tert-Butylamino)-3-hydroxy-3-phenylpropanenitrile (9): To a test tube was added 8 (64.8 mg, $0.30 \mathrm{mmol}), \mathrm{NaBH}_{4}(13.6 \mathrm{mg}, 0.36 \mathrm{mmol}), \mathrm{CH}_{3} \mathrm{OH}(1.2 \mathrm{~mL})$ and THF (1.8 mL). The mixture was then stirred at $0{ }^{\circ} \mathrm{C}$ for 10 min until the reaction was completed (monitored by TLC). Upon completion, the reaction mixture was extracted with ethyl acetate. The organic layer was then washed with $\mathrm{H}_{2} \mathrm{O}$ and brine, dried over anhydrous $\mathrm{Na}_{2} \mathrm{SO}_{4}$, filtered and concentrated in vacuo. Then, the residue was purified by column chromatography on silica gel (petroleum ether/ethyl acetate $=5: 1, \mathrm{v} / \mathrm{v})$ to give the desired product 9 as a white solid $(60.8 \mathrm{mg}, 93 \%$ yield $)$ and the $\mathrm{dr}$ of 
9 was determined to be $>20: 1$ by NMR spectrum of crude product. m.p.: $71-72{ }^{\circ} \mathrm{C}$; IR $\left(\mathrm{KBr}, \mathrm{cm}^{-1}\right)$ : 3214, 2973, 1598, 1382, 1227, 1099, 819, 703; ${ }^{1} \mathrm{H}$ NMR (Acetone- $d_{6}, 500 \mathrm{MHz}$ ): $\delta 7.49$ (d, $J=$ $7.4 \mathrm{~Hz}, 2 \mathrm{H}), 7.35$ (t, $J=7.4 \mathrm{~Hz}, 2 \mathrm{H}), 7.29(\mathrm{t}, J=7.3 \mathrm{~Hz}, 1 \mathrm{H}), 5.09(\mathrm{~d}, J=4.8 \mathrm{~Hz}, 1 \mathrm{H}), 4.81(\mathrm{t}, J=$ $4.9 \mathrm{~Hz}, 1 \mathrm{H}), 3.83(\mathrm{~s}, 1 \mathrm{H}), 2.89(\mathrm{~s}, 1 \mathrm{H}), 1.12(\mathrm{~s}, 9 \mathrm{H}) ;{ }^{13} \mathrm{C}$ NMR (Acetone- $\left.d_{6}, 125 \mathrm{MHz}\right): \delta 141.9$, 128.7, 128.5, 127.6, 121.7, 75.9, 52.6, 51.6, 29.5; LC-MS (ESI) $\mathrm{m} / z 219$ [M+H] $]^{+}$; HRMS (ESI) $m / z$ calcd for $\mathrm{C}_{13} \mathrm{H}_{19} \mathrm{ON}_{2}[\mathrm{M}+\mathrm{H}]^{+}$219.1492, found 219.1491 .<smiles>CC(C)CNC(C#N)[C@@H](O)c1ccccc1</smiles>

9

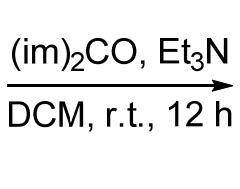<smiles>CC(C)N1C(=O)O[C@H](c2ccccc2)[C@H]1C#N</smiles>

$10, \mathrm{dr}>20: 1$

cis-3-(tert-Butyl)-2-oxo-5-phenyloxazolidine-4-carbonitrile (10): To a test tube was added 9 $(65.5 \mathrm{mg}, 0.30 \mathrm{mmol})$ and dichloromethane $(3.0 \mathrm{~mL})$. Followed by the addition of $\mathrm{Et}_{3} \mathrm{~N}(42 \mu \mathrm{L}$, $0.30 \mathrm{mmol}),(\mathrm{Im})_{2} \mathrm{CO}(97.3 \mathrm{mg}, 0.60 \mathrm{mmol})$. The mixture was then stirred at r.t. for $12 \mathrm{~h}$ until the reaction was completed (monitored by TLC). Upon completion, the reaction mixture was extracted with dichloromethane. The organic layer was then washed with $0.1 \mathrm{M} \mathrm{HCl}(3.0 \mathrm{~mL})$ and $\mathrm{H}_{2} \mathrm{O}\left(7.0 \mathrm{~mL}\right.$ ), dried over anhydrous $\mathrm{Na}_{2} \mathrm{SO}_{4}$, filtered and concentrated in vacuo. The residue was purified by column chromatography on silica gel (petroleum ether/ethyl acetate $=5: 1, \mathrm{v} / \mathrm{v}$ ) to give the desired product $\mathbf{1 0}$ as a white solid (67.9 $\mathrm{mg}, 93 \%$ yield) and the cis configuration of $\mathbf{1 0}$ was assigned by NOE experiment. m.p.: $125-126{ }^{\circ} \mathrm{C}$; IR (KBr, $\left.\mathrm{cm}^{-1}\right): 2979,2237,1761,1371,1208$, 1029, 769, 719; ${ }^{1} \mathrm{H}$ NMR (Acetone- $\left.d_{6}, 500 \mathrm{MHz}\right): \delta 7.48-7.41(\mathrm{~m}, 5 \mathrm{H}), 5.56$ (d, $\left.J=7.3 \mathrm{~Hz}\right), 4.83$ $\left(\mathrm{d}, J=7.3 \mathrm{~Hz}\right.$ ), 1.56 (s, 9H); ${ }^{13} \mathrm{C}$ NMR (Acetone- $d_{6}, 125 \mathrm{MHz}$ ): $\delta$ 154.8, 132.5, 130.1, 129.2, 126.1, 115.2, 75.6, 55.8, 53.4, 27.7; LC-MS (ESI) $m / z 245$ [M+H] $]^{+}$; HRMS (ESI) $m / z$ calcd for $\mathrm{C}_{14} \mathrm{H}_{17} \mathrm{O}_{2} \mathrm{~N}_{2}[\mathrm{M}+\mathrm{H}]^{+}$245.1285, found 245.1283.

\section{X-ray Crystallographic Data for Compounds 2i}<smiles>CC(C)/N=C(\C#N)C(=O)c1ccc(Br)cc1</smiles>

2i

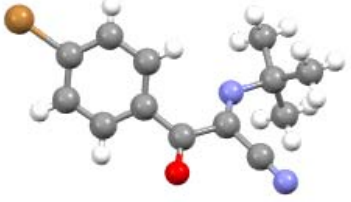

X-ray structure of $\mathbf{2 i}$

Crystallographic data for 2i: $\mathrm{C}_{13} \mathrm{H}_{13} \mathrm{~N}_{2} \mathrm{OBr}, \mathrm{M}=293.163$, orthorhombic, P 212121 (No. 19), $\mathrm{a}=$ 7.192(16) $\AA, \mathrm{b}=8.707(19) \AA, \mathrm{c}=22.81(5) \AA, \mathrm{V}=1428(5) \AA^{3}, \mathrm{Z}=4$, Crystal size: $0.25 \times 0.20 \times 0.14 \mathrm{~mm}, \mathrm{~T}=295 \mathrm{~K}, \rho_{\text {calcd }}=1.363 \mathrm{~g} \cdot \mathrm{cm}^{-3}, \mathrm{R}_{1}=0.0373(\mathrm{I}>4 \sigma(\mathrm{I})), \mathrm{wR}_{2}=0.0906$ (all data), GOF $=1.029$, reflections collected/unique: $8100 / 1245($ Rint $=0.0497)$, Data: 1800 , restraints: 0, parameters: 155 . CCDC 1913844 contains the supplementary crystallographic data for this paper. The data can be obtained free of charge from The Cambridge Crystallographic Data Centre via www.ccdc.cam.ac.uk/data_request/cif. 


\section{Mechanistic Studies}

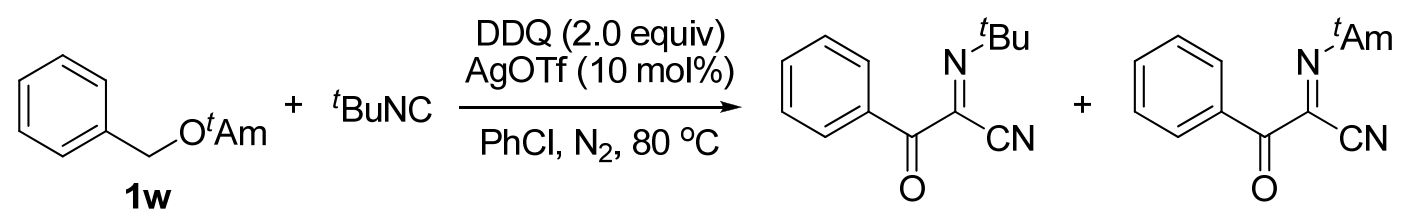

2a, $72 \%$

2a' (not observed)

To a test tube, the mixture of DDQ (136.2 mg, $0.6 \mathrm{mmol})$, AgOTf (7.8 mg, $0.03 \mathrm{mmol})$ and PhCl $(3.0 \mathrm{~mL})$ was added $\mathbf{1} \mathbf{w}^{13}(53.5 \mathrm{mg}, 0.3 \mathrm{mmol})$ and ${ }^{t} \mathrm{BuNC}(136 \mu \mathrm{L}, 1.2 \mathrm{mmol})$. The mixture was stirred at $80{ }^{\circ} \mathrm{C}$ under $\mathrm{N}_{2}$ as monitored by TLC. Upon completion, the reaction mixture was cooled down to room temperature and concentrated under vacuum. The residue was purified by column chromatography on silica gel (petroleum ether/ethyl acetate $=100: 1, \mathrm{v} / \mathrm{v}$ ) to give $\mathbf{2 a}$ as a light yellow solid (46.5 mg, 72\% yield).

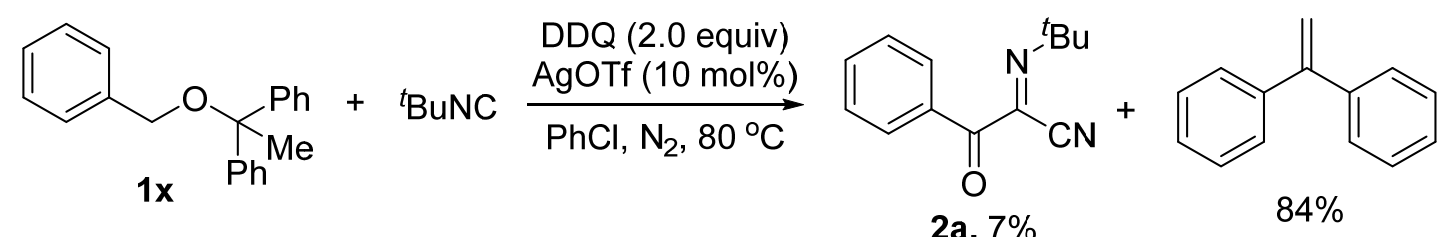

To a test tube, the mixture of DDQ (136.2 $\mathrm{mg}, 0.6 \mathrm{mmol}), \operatorname{AgOTf}(7.8 \mathrm{mg}, 0.03 \mathrm{mmol})$ and $\mathrm{PhCl}$ $(3.0 \mathrm{~mL})$ was added $1 \times$ ( $(53.5 \mathrm{mg}, 0.3 \mathrm{mmol})$ and ${ }^{t} \mathrm{BuNC}(136 \mu \mathrm{L}, 1.2 \mathrm{mmol})$. The mixture was stirred at $80{ }^{\circ} \mathrm{C}$ under $\mathrm{N}_{2}$ as monitored by TLC. Upon completion, the reaction mixture was cooled down to room temperature and concentrated under vacuum. The residue was purified by column chromatography on silica gel (petroleum ether/ethyl acetate $=100: 1, \mathrm{v} / \mathrm{v}$ ) to give $\mathbf{2 a}$ as a light yellow solid (4.6 mg, 7\% yield) and 1,1-diphenylethylene ( $45.5 \mathrm{mg}, 84 \%$ yield).
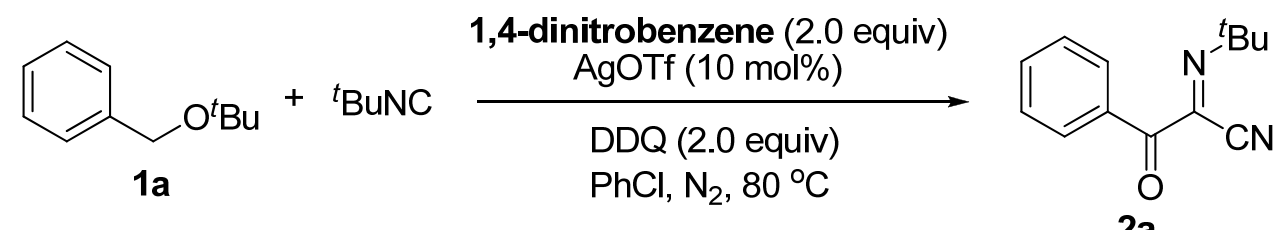

2a

To a test tube, the mixture of DDQ (136.2 mg, $0.6 \mathrm{mmol})$, AgOTf $(7.8 \mathrm{mg}, 0.03 \mathrm{mmol})$, 1,4-dinitrobenzene $(100.9 \mathrm{mg}, 0.6 \mathrm{mmol})$ and $\mathrm{PhCl}(3.0 \mathrm{~mL})$ was added $\mathbf{1 a}(53 \mu \mathrm{L}, 0.3 \mathrm{mmol})$ and ${ }^{t} \mathrm{BuNC}(136 \mu \mathrm{L}, 1.2 \mathrm{mmol})$. The mixture was stirred at $80{ }^{\circ} \mathrm{C}$ under $\mathrm{N}_{2}$ as monitored by TLC. Upon completion, the reaction mixture was cooled down to room temperature and concentrated under vacuum. The residue was purified by column chromatography on silica gel (petroleum ether/ethyl acetate $=100: 1, \mathrm{v} / \mathrm{v})$ to give $\mathbf{2 a}$ as a light yellow solid $(52.5 \mathrm{mg}, 81 \%$ yield $)$.

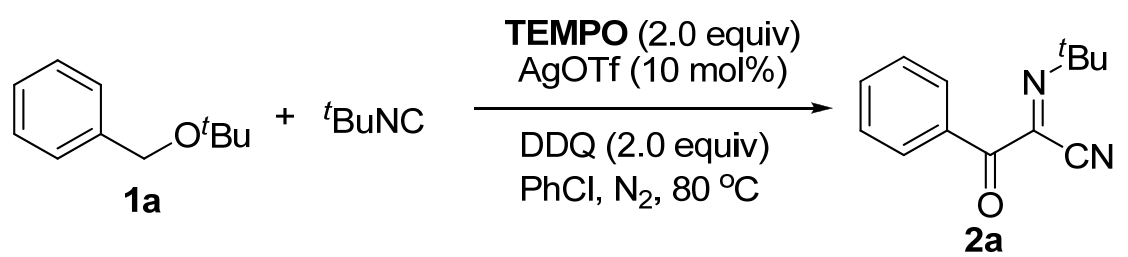

To a test tube, the mixture of DDQ (136.2 $\mathrm{mg}, 0.6 \mathrm{mmol})$, AgOTf (7.8 mg, $0.03 \mathrm{mmol})$, TEMPO (93.8 mg, $0.6 \mathrm{mmol})$ and $\mathrm{PhCl}(3.0 \mathrm{~mL})$ was added $1 \mathrm{a}(53 \mu \mathrm{L}, 0.3 \mathrm{mmol})$ and ${ }^{t} \mathrm{BuNC}(136 \mu \mathrm{L}, 1.2$ 
mmol). The mixture was stirred at $80{ }^{\circ} \mathrm{C}$ under $\mathrm{N}_{2}$ as monitored by TLC. Upon completion, the reaction mixture was cooled down to room temperature and concentrated under vacuum. The residue was purified by column chromatography on silica gel (petroleum ether/ethyl acetate $=$ $100: 1, \mathrm{v} / \mathrm{v}$ ) to give $\mathbf{2 a}$ as a light yellow solid (32.5 $\mathrm{mg}, 51 \%$ yield).

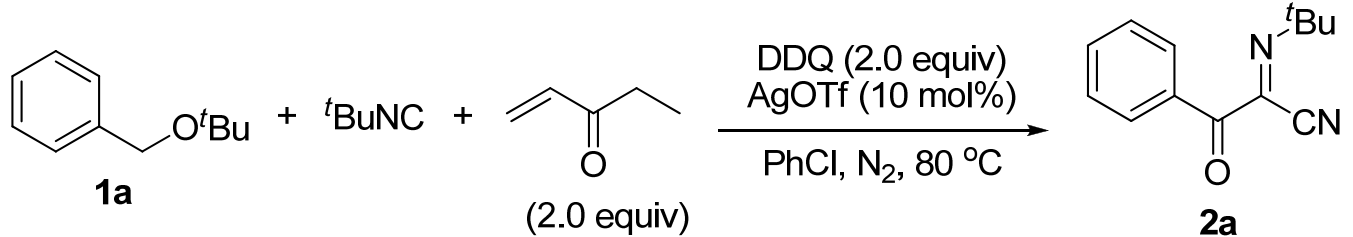

To a test tube, the mixture of DDQ (136.2 $\mathrm{mg}, 0.6 \mathrm{mmol})$, AgOTf $(7.8 \mathrm{mg}, 0.03 \mathrm{mmol}), 1$ penten-3-one $(50.5 \mathrm{mg}, 0.6 \mathrm{mmol})$ and $\mathrm{PhCl}(3.0 \mathrm{~mL})$ was added $1 \mathrm{a}(53 \mu \mathrm{L}, 0.3 \mathrm{mmol})$ and ${ }^{t} \mathrm{BuNC}(136 \mu \mathrm{L}, 1.2 \mathrm{mmol})$. The mixture was stirred at $80^{\circ}$ under $\mathrm{N}_{2}$ as monitored by TLC. Upon completion, the reaction mixture was cooled down to room temperature and concentrated under vacuum. The residue was purified by column chromatography on silica gel (petroleum ether/ethyl acetate $=100: 1, \mathrm{v} / \mathrm{v}$ ) to give $\mathbf{2 a}$ as a light yellow solid (38.9 $\mathrm{mg}, 61 \%$ yield).

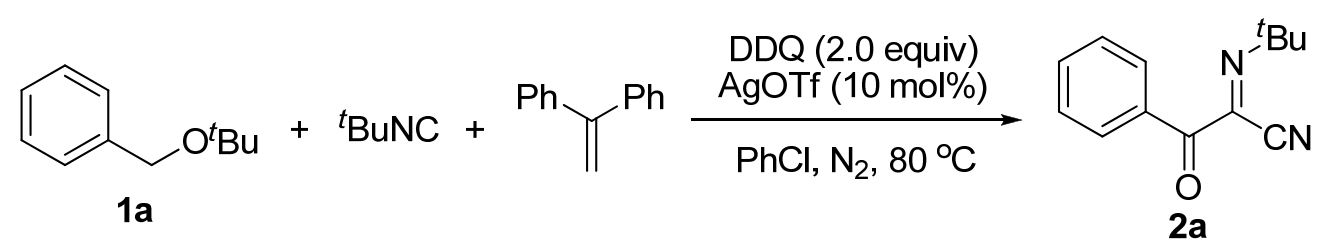

To a test tube, the mixture of DDQ (136.2 mg, $0.6 \mathrm{mmol})$, AgOTf $(7.8 \mathrm{mg}, 0.03 \mathrm{mmol})$, 1,1-diphenylethylene $(108.2 \mathrm{mg}, 0.6 \mathrm{mmol})$ and $\mathrm{PhCl}(3.0 \mathrm{~mL})$ was added $1 \mathrm{a}(53 \mu \mathrm{L}, 0.3 \mathrm{mmol})$ and ${ }^{t} \mathrm{BuNC}(136 \mu \mathrm{L}, 1.2 \mathrm{mmol})$. The mixture was stirred at $80{ }^{\circ} \mathrm{C}$ under $\mathrm{N}_{2}$ as monitored by TLC. Upon completion, the reaction mixture was cooled down to room temperature and concentrated under vacuum. The residue was purified by column chromatography on silica gel (petroleum ether/ethyl acetate $=100: 1, \mathrm{v} / \mathrm{v}$ ) to give $\mathbf{2 a}$ as a light yellow solid (39.8 $\mathrm{mg}, 62 \%$ yield).

\section{References}

[1] Mayeda, E. A.; Miller, L. L.; Wolf, J. F. J. Am. Chem. Soc. 1972, 94, 6812.

[2] Billups, W. E.; Reed, L. E.; Casserly, E. W.; Lin, L. P. J. Org. Chem. 1981, 46, 1326.

[3] Cristel, S. J.; Bindel, T. H. J. Org. Chem. 1980, 45, 951.

[4] Roof, A. A. M.; Woerden, H. F. V.; Cerfontain, H. Tetrahedron 1976, 32, 2967.

[5] Neuman, R. C.; Behar, J. V. J. Org. Chem. 1971, 36, 657.

[6] Wang, L.; Hashidoko, Y.; Hashimoto, M. J. Org. Chem. 2016, 81, 4464.

[7] Han, H.; Janda, K. D. J. Am. Chem. Soc. 1996, 118, 2539.

[8] Eißler, S.; Bogner, T.; Nahrwold, M.; Sewald, N. Chem. Eur. J. 2009, 15, 11273.

[9] Bos, M. E.; Wulff, W. D.; Miller, R. A.; Chamberlin, S.; Brandvold, T. A. J. Am. Chem. Soc. 1991, 113, 9293.

[10] Shirinia, F.; Zolfigolb, M. A.; Abedini, M. J. Iran. Chem. Soc. 2010, 7, 603.

[11] Guan, B.; Xiang, S.; Wang, B.; Sun, Z.; Wang, Y.; Zhao, K.; Shi, Z. J. Am. Chem. Soc. 2008, 130,3268 .

[12] Lassalas, P.; Marsais, F.; Hoarau, C. Synlett 2013, 24, 2233.

[13] Figadere, B.; Franck, X.; Cave, A. Tetrahedron Lett. 1993, 34, 5893. 
Copies of ${ }^{1} \mathrm{H},{ }^{13} \mathrm{C}$ and ${ }^{19} \mathrm{~F}$ NMR Spectra

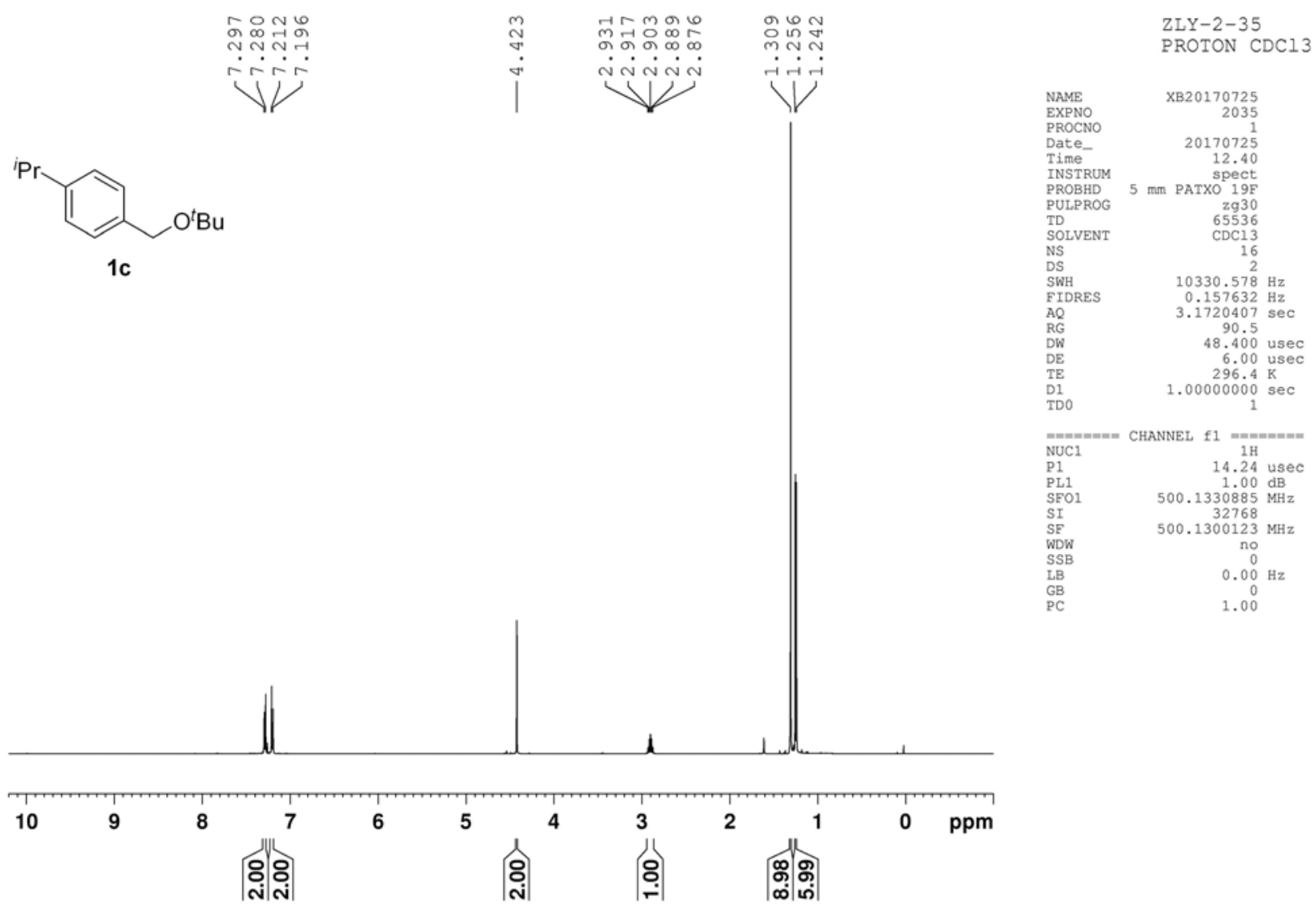




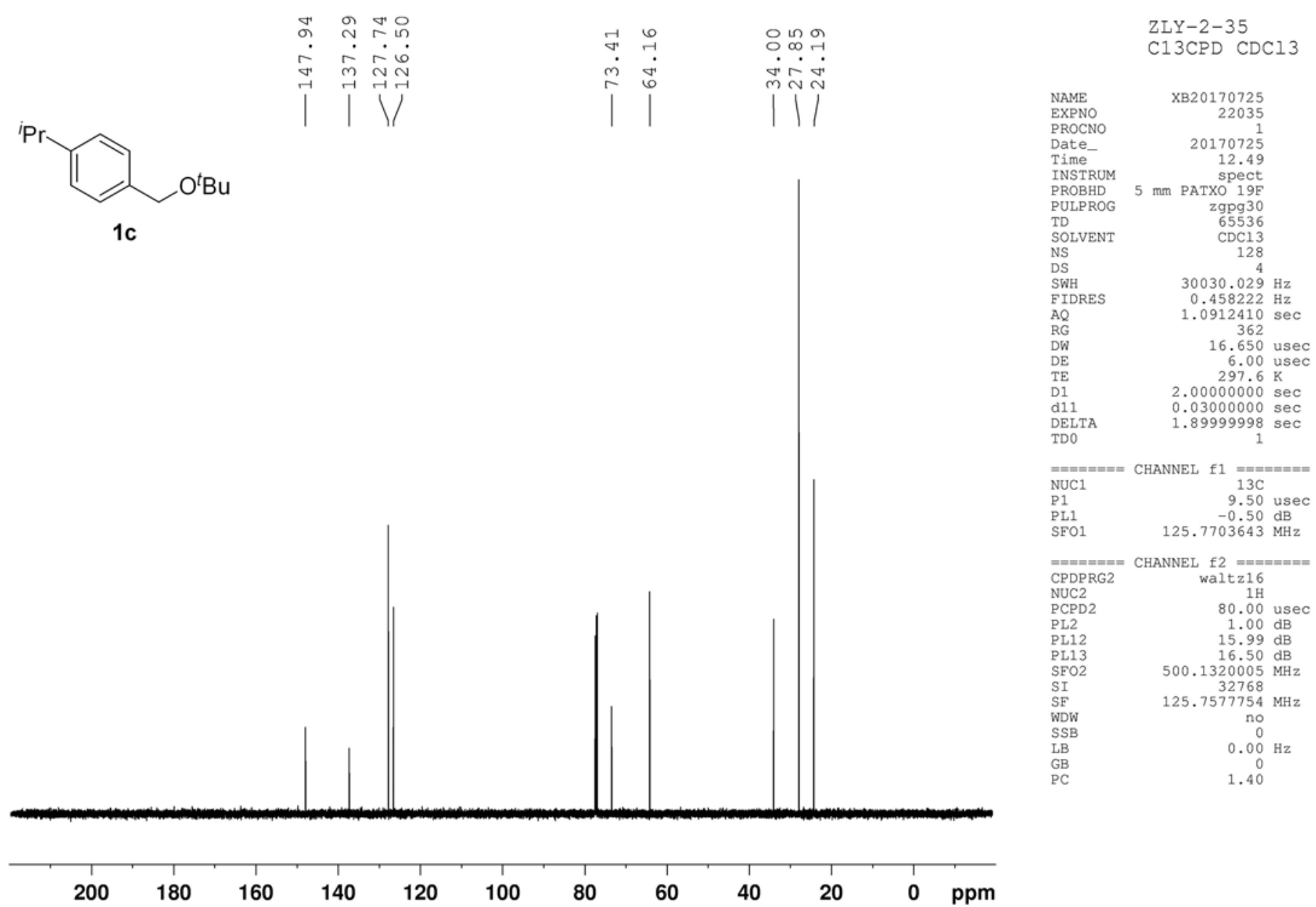




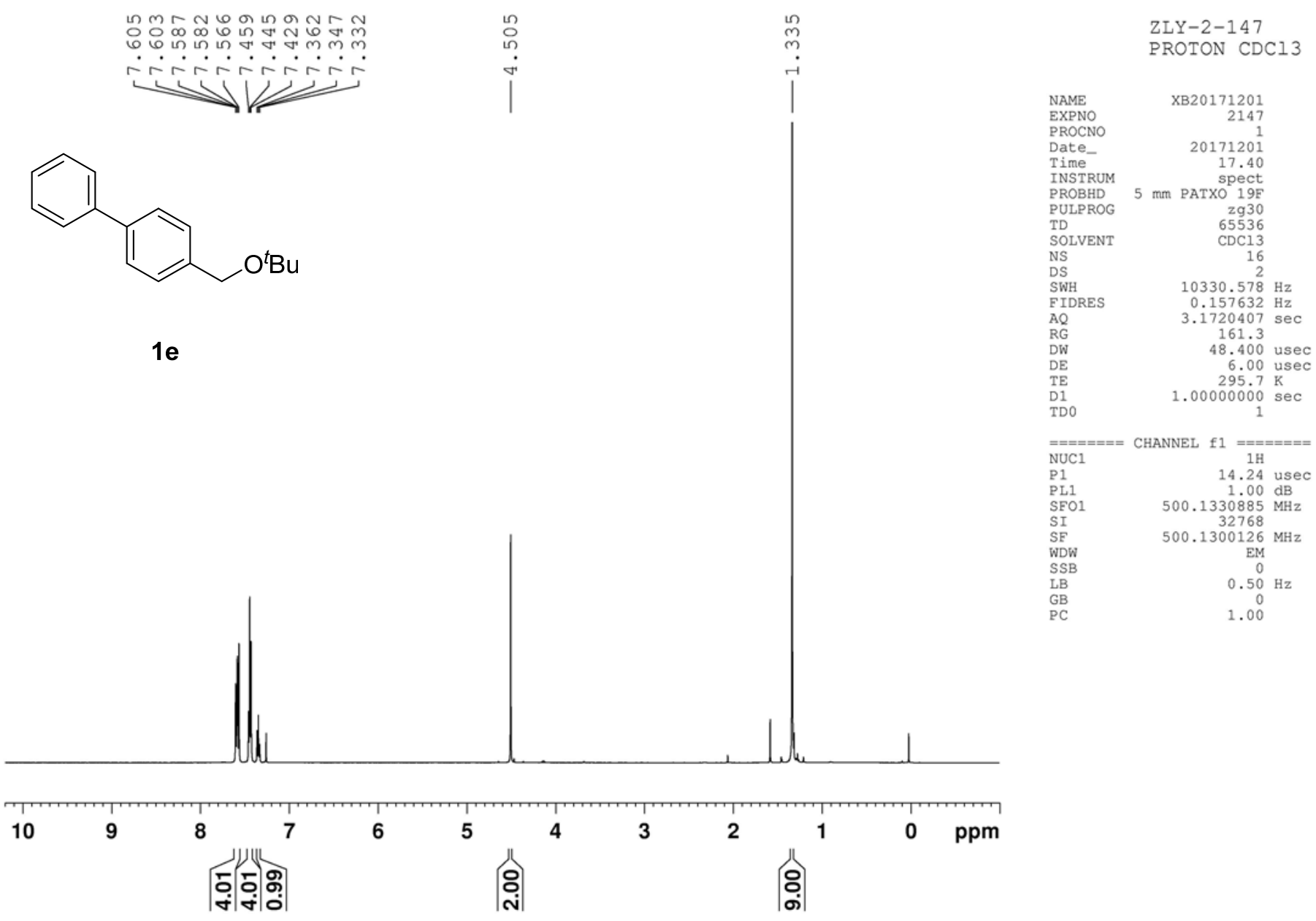




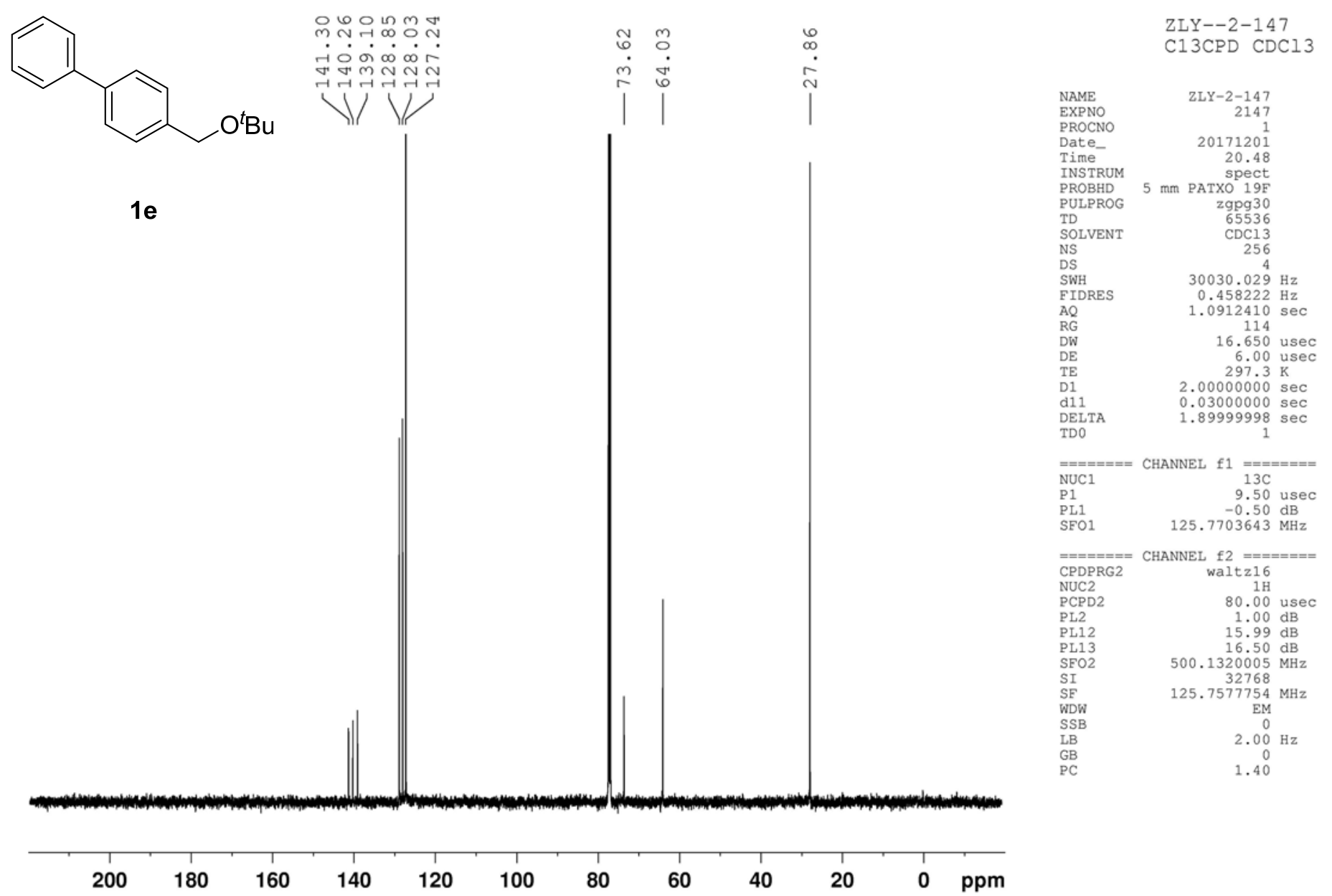




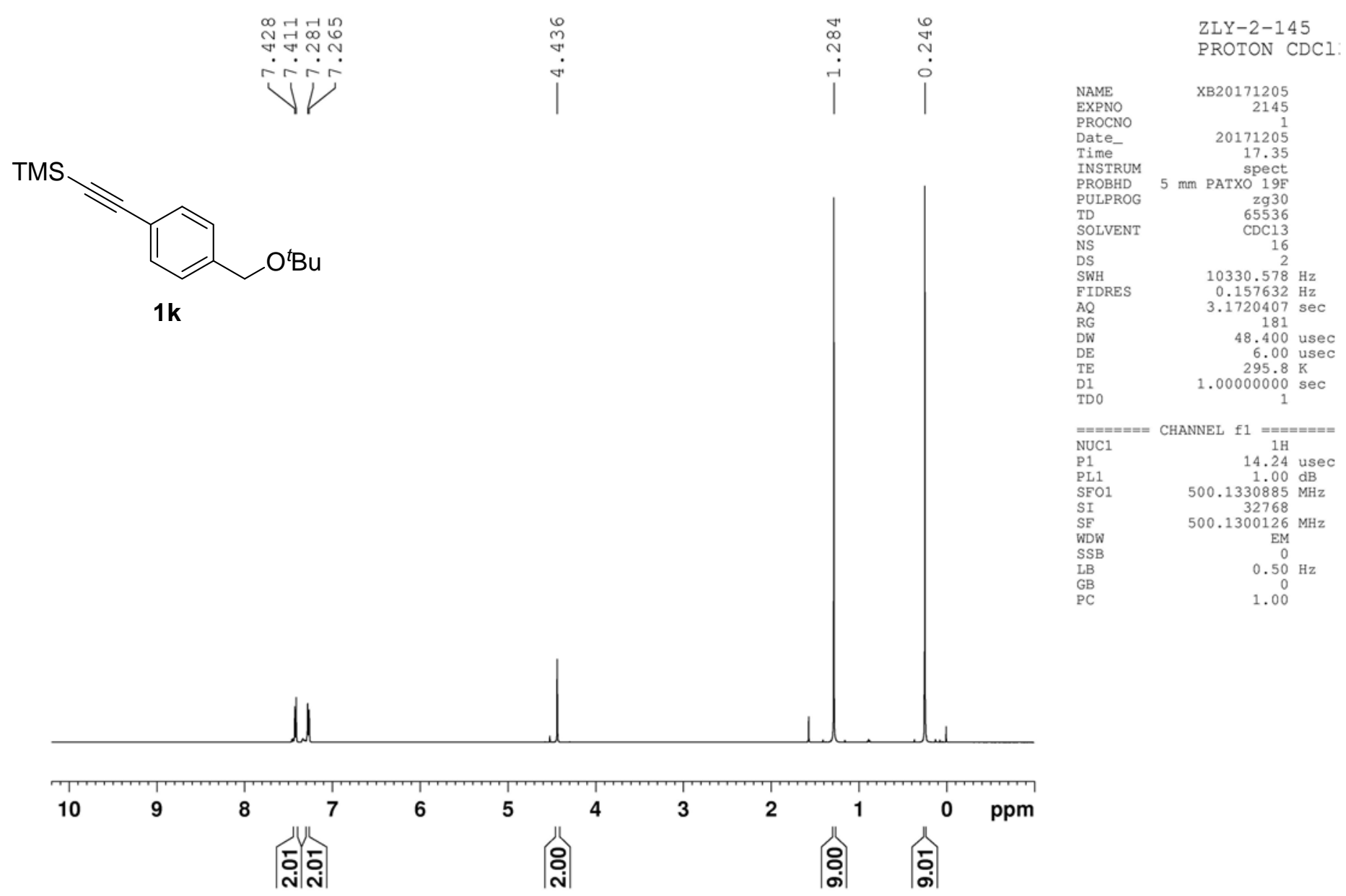


TMS

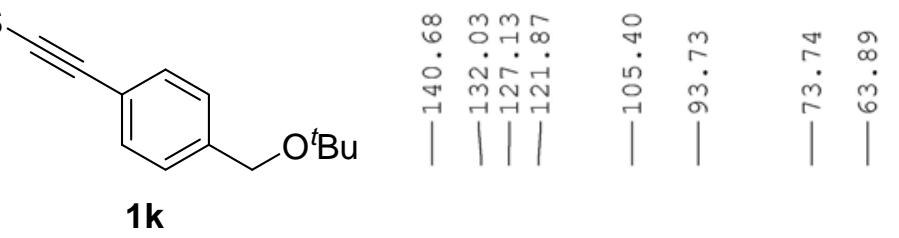

$1 k$

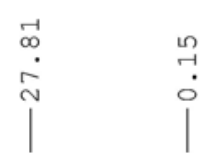
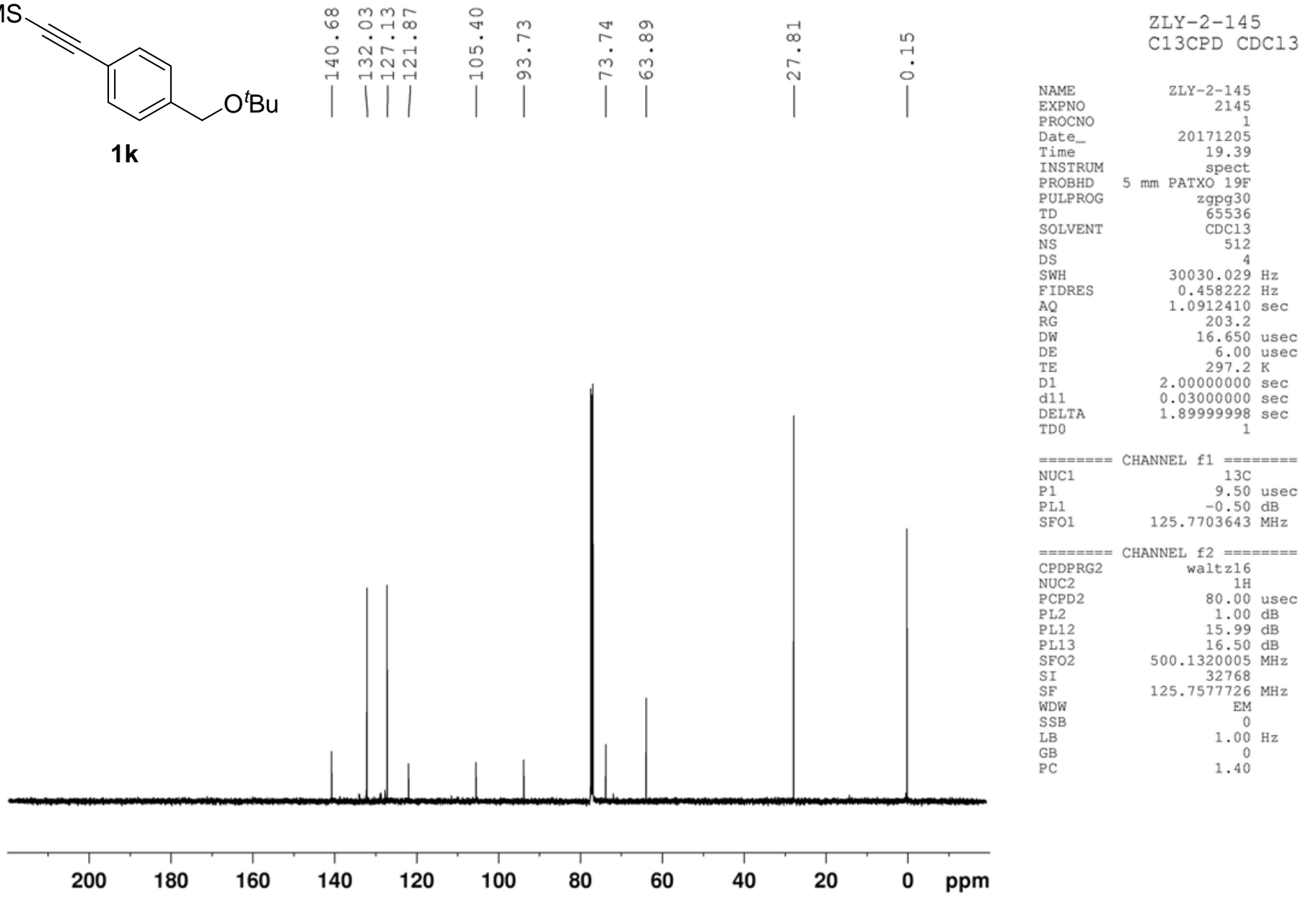


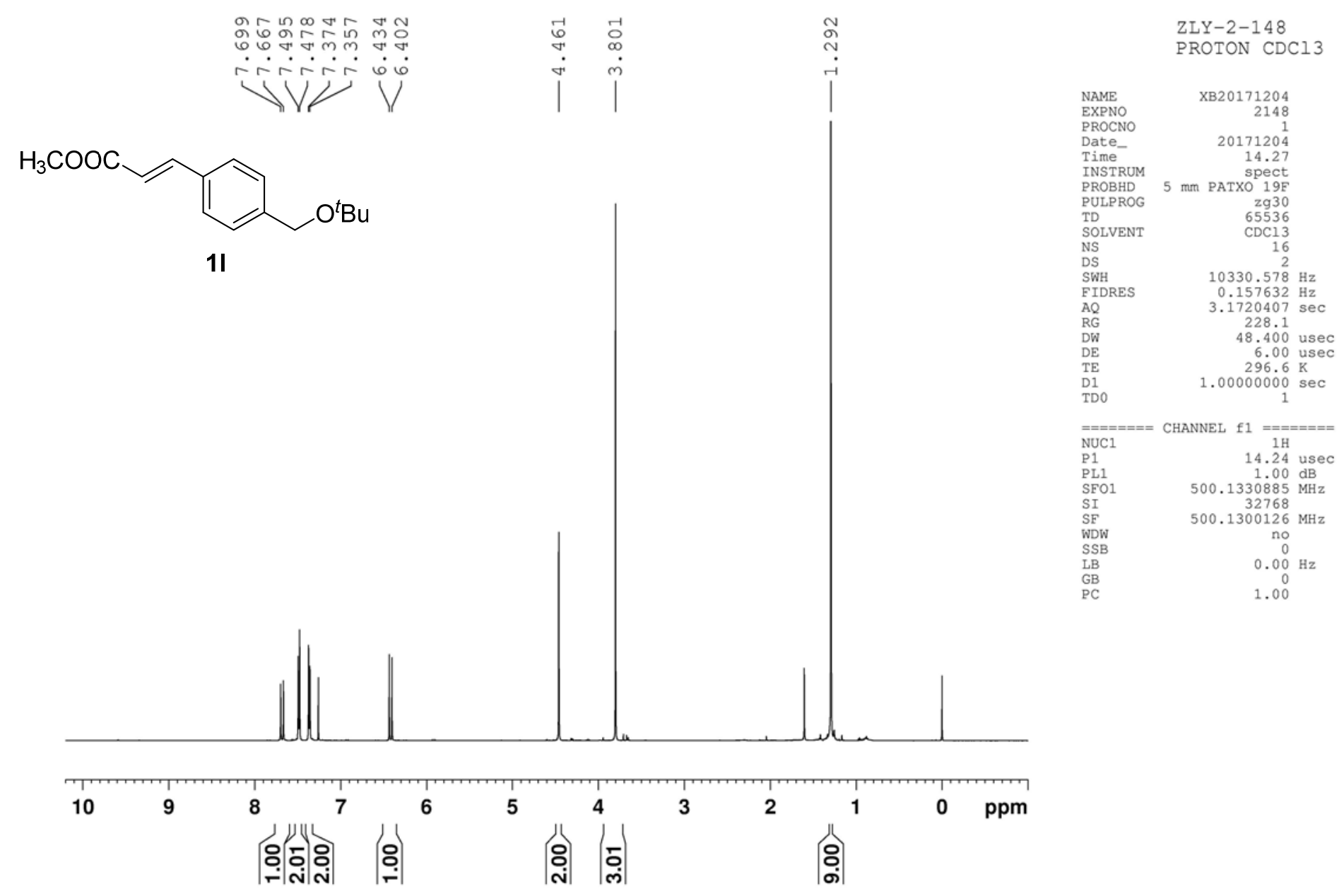




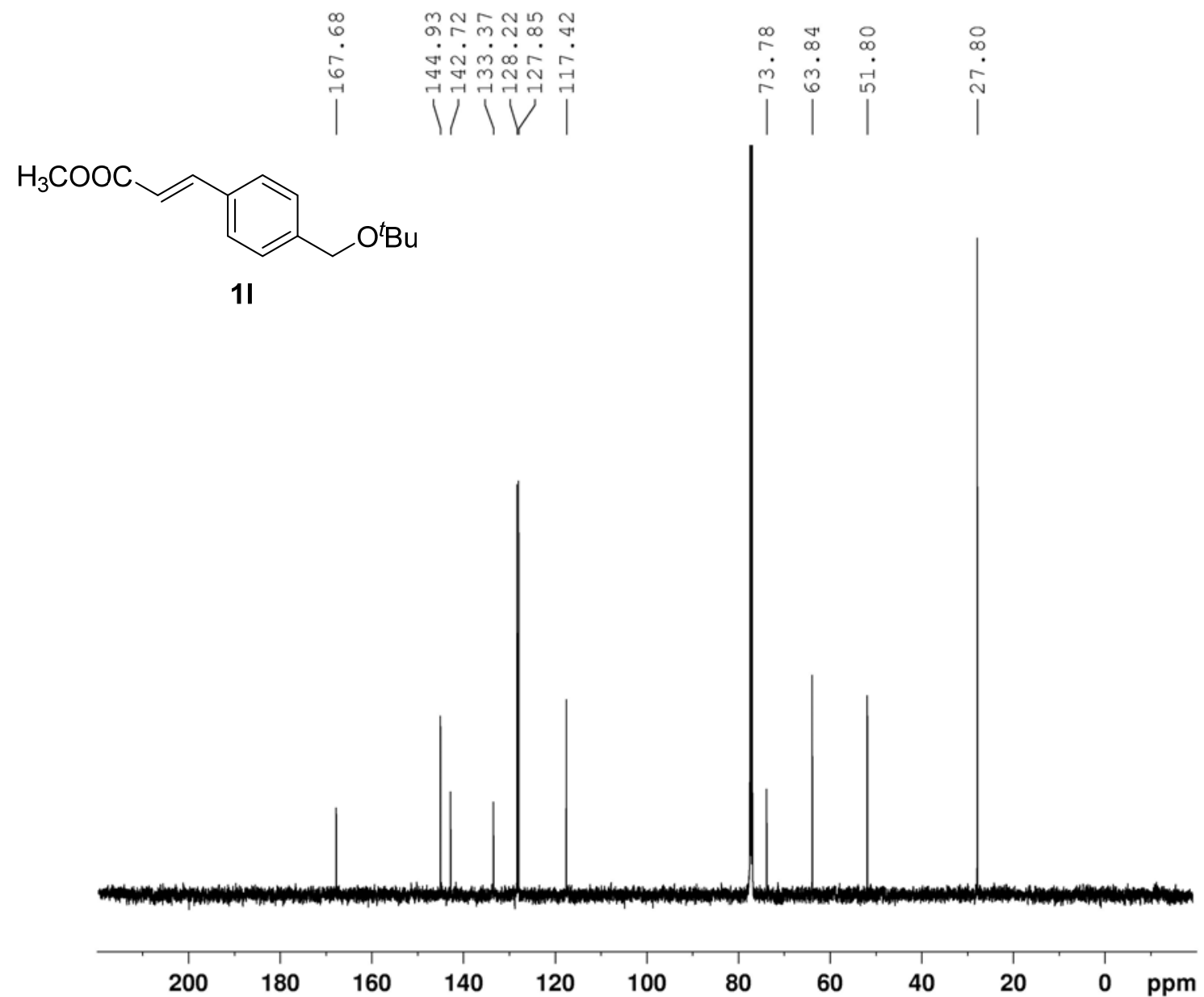




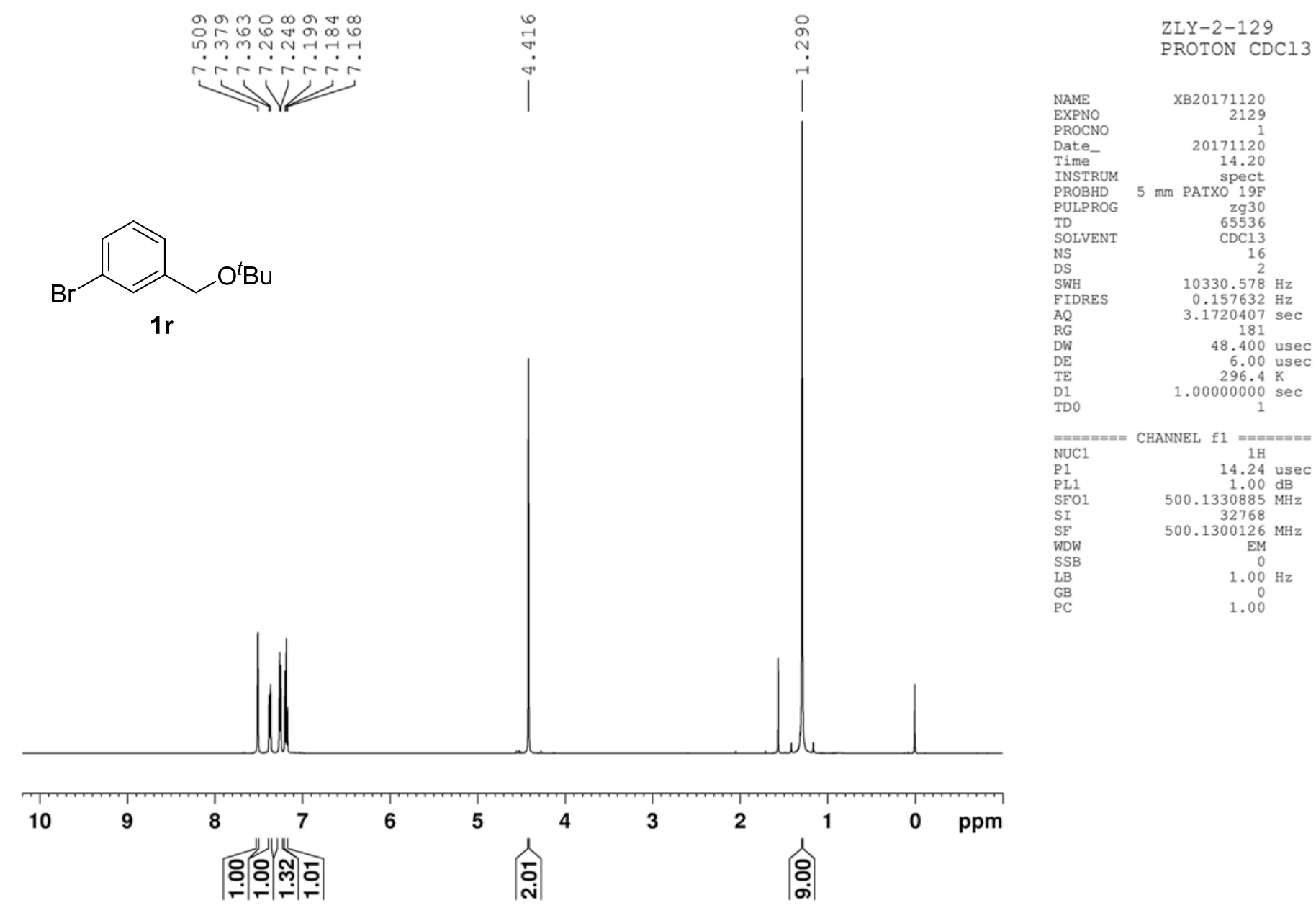



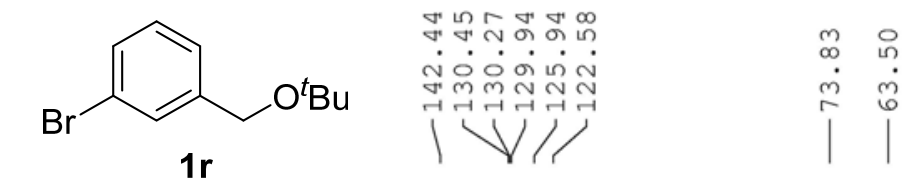

ZLY-2-129

C13CPD CDC13

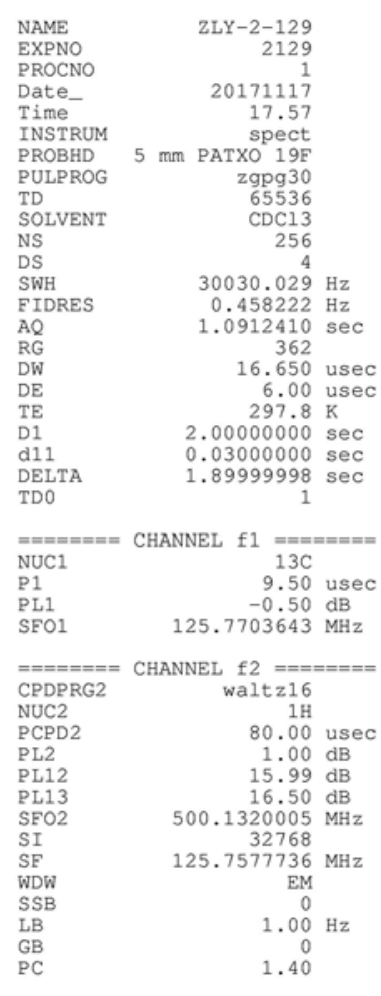




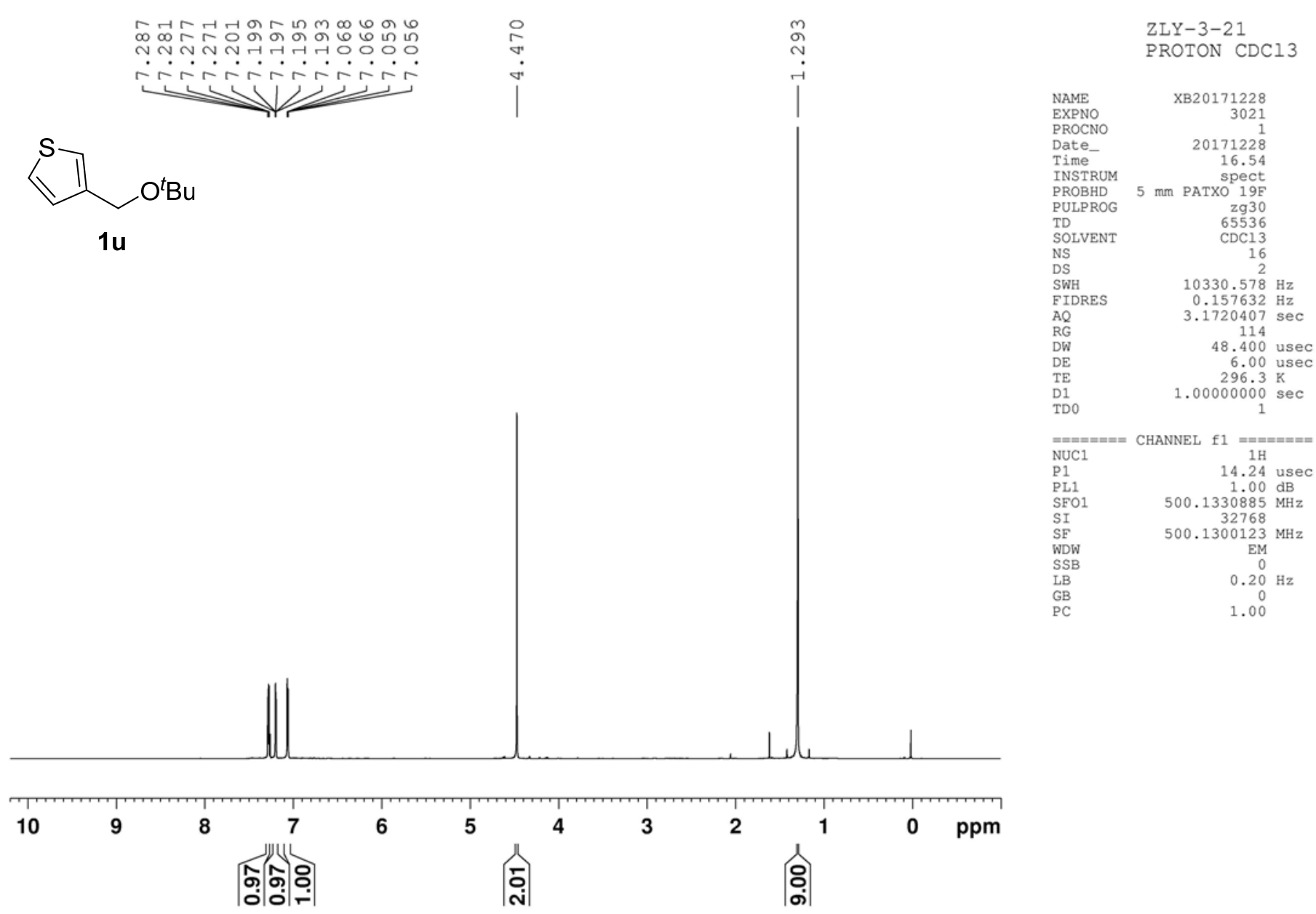




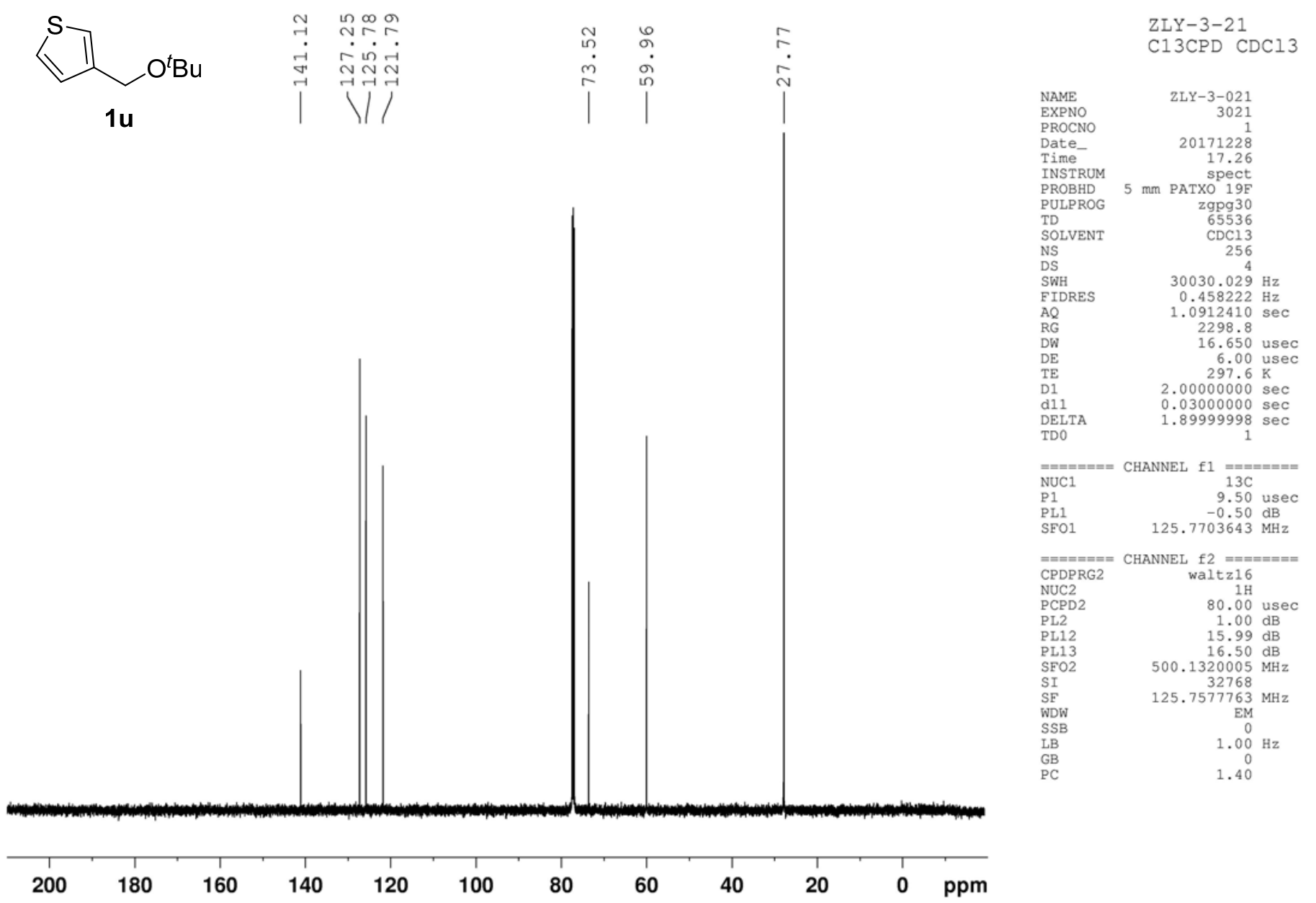




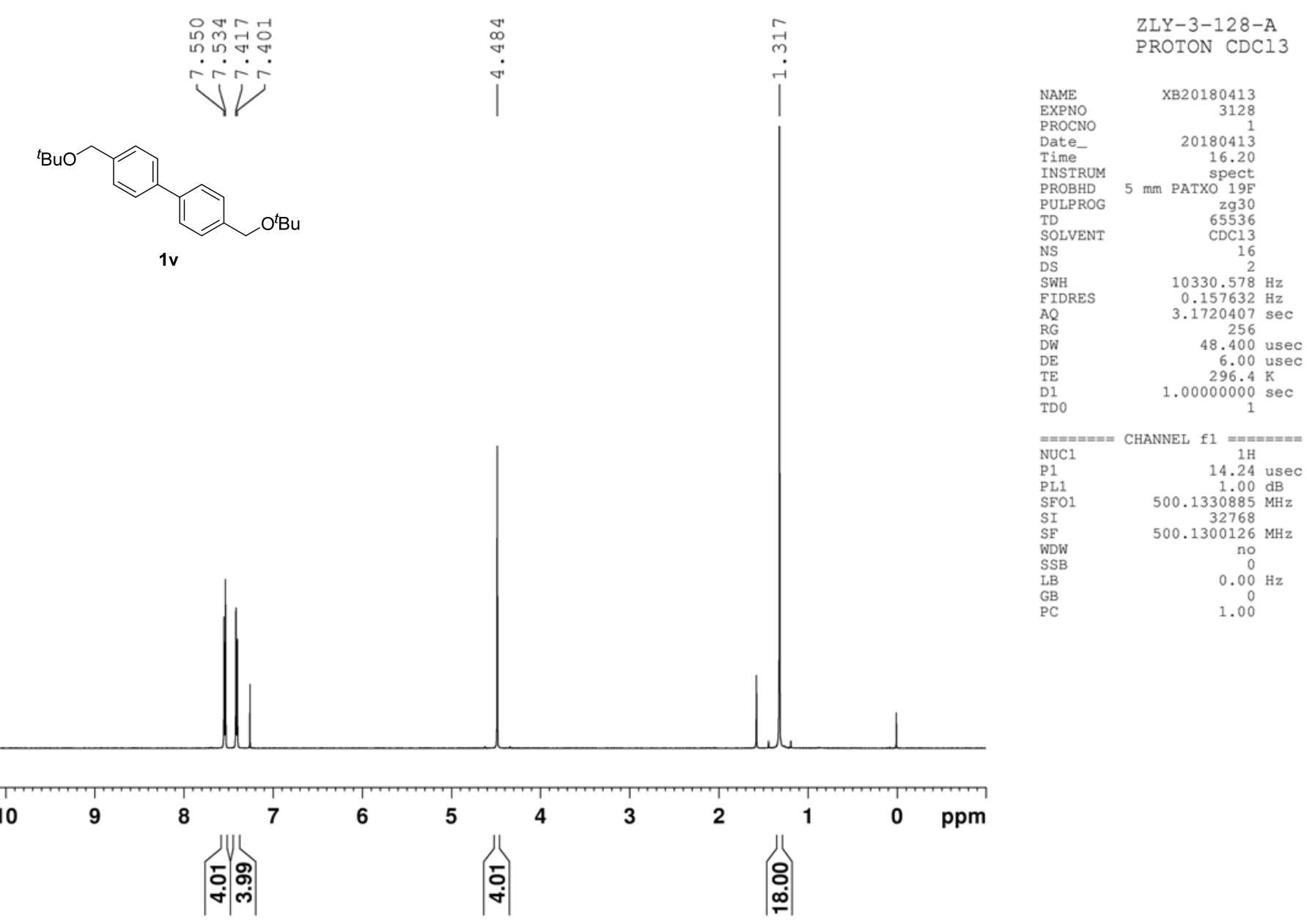




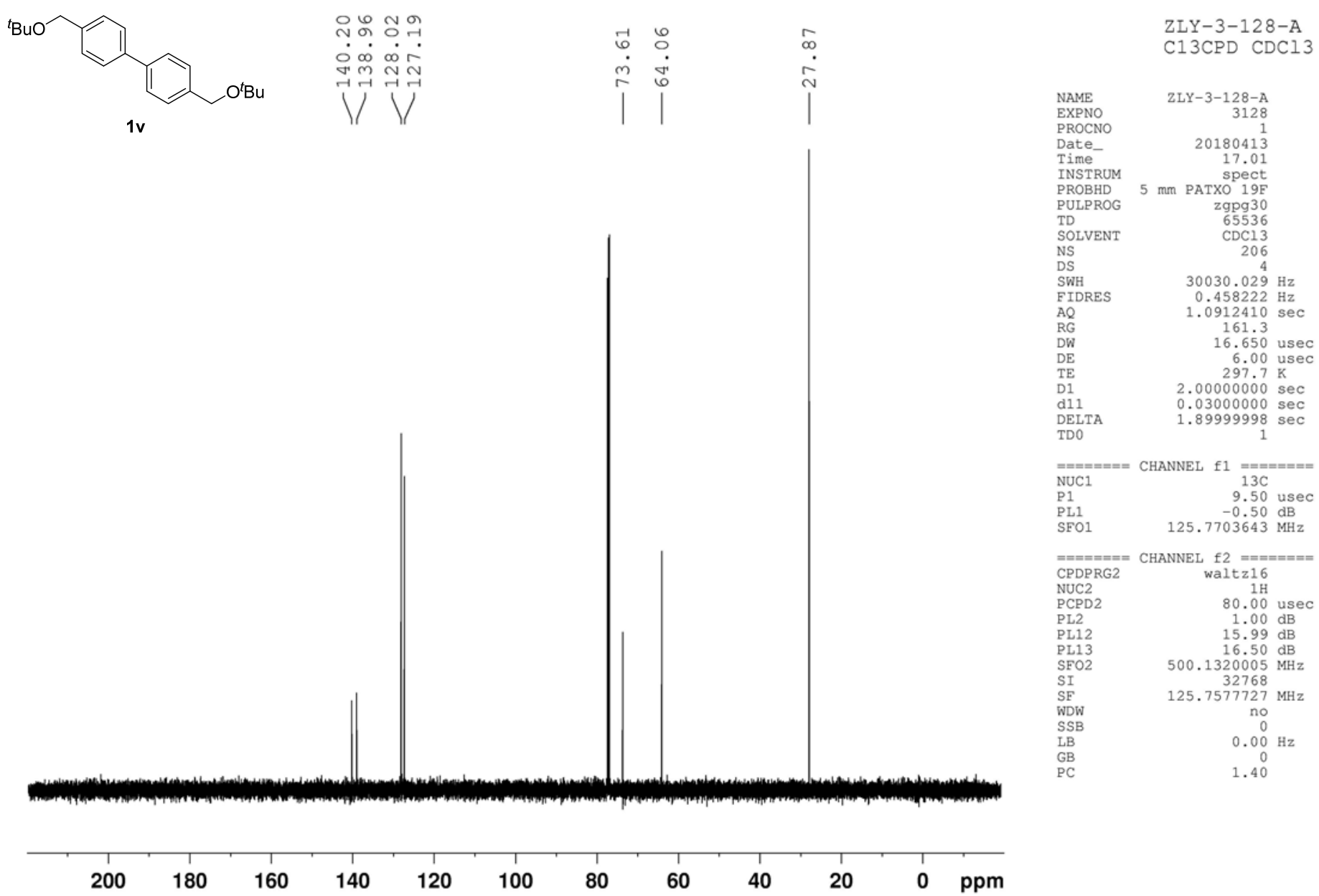




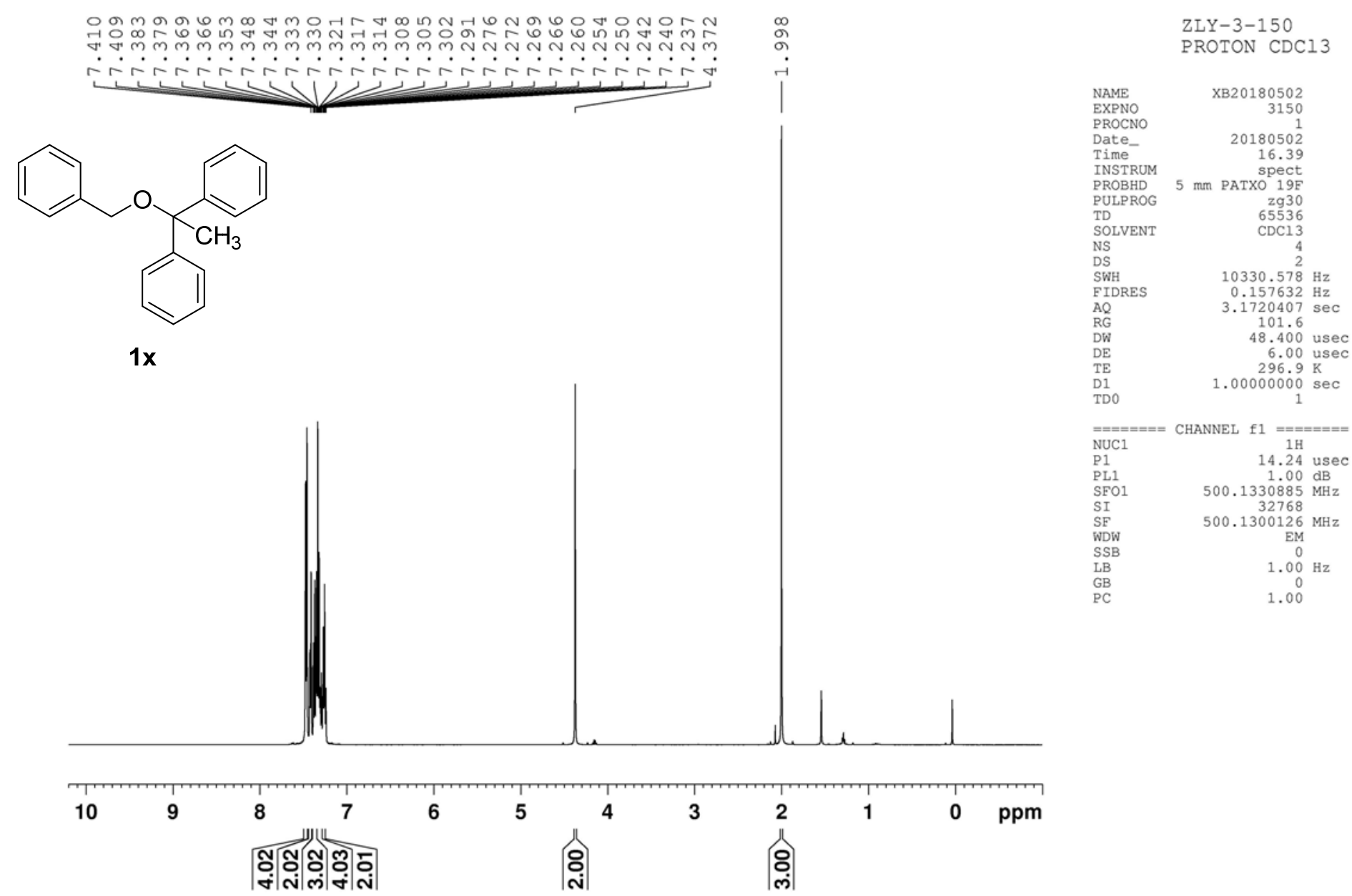




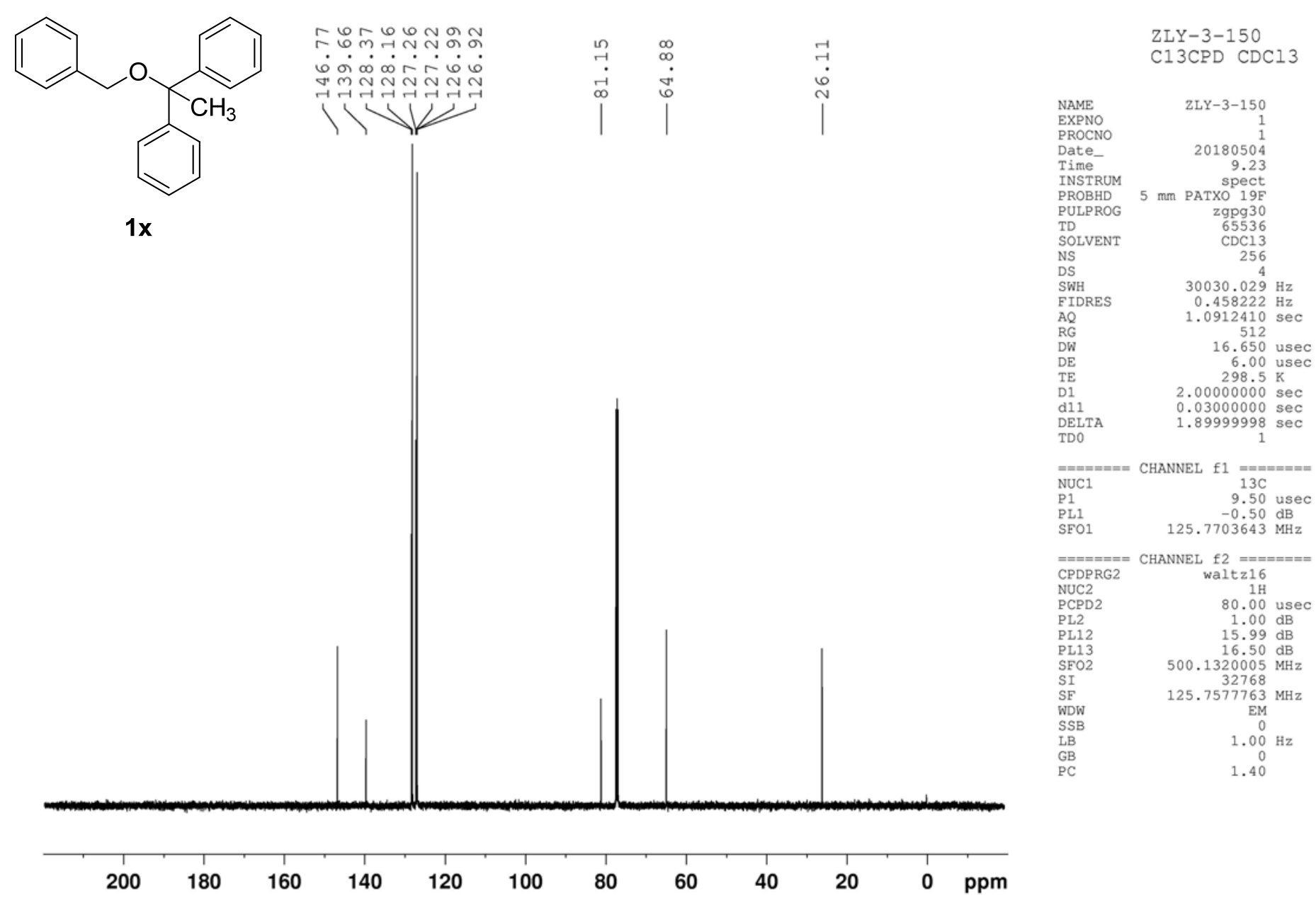




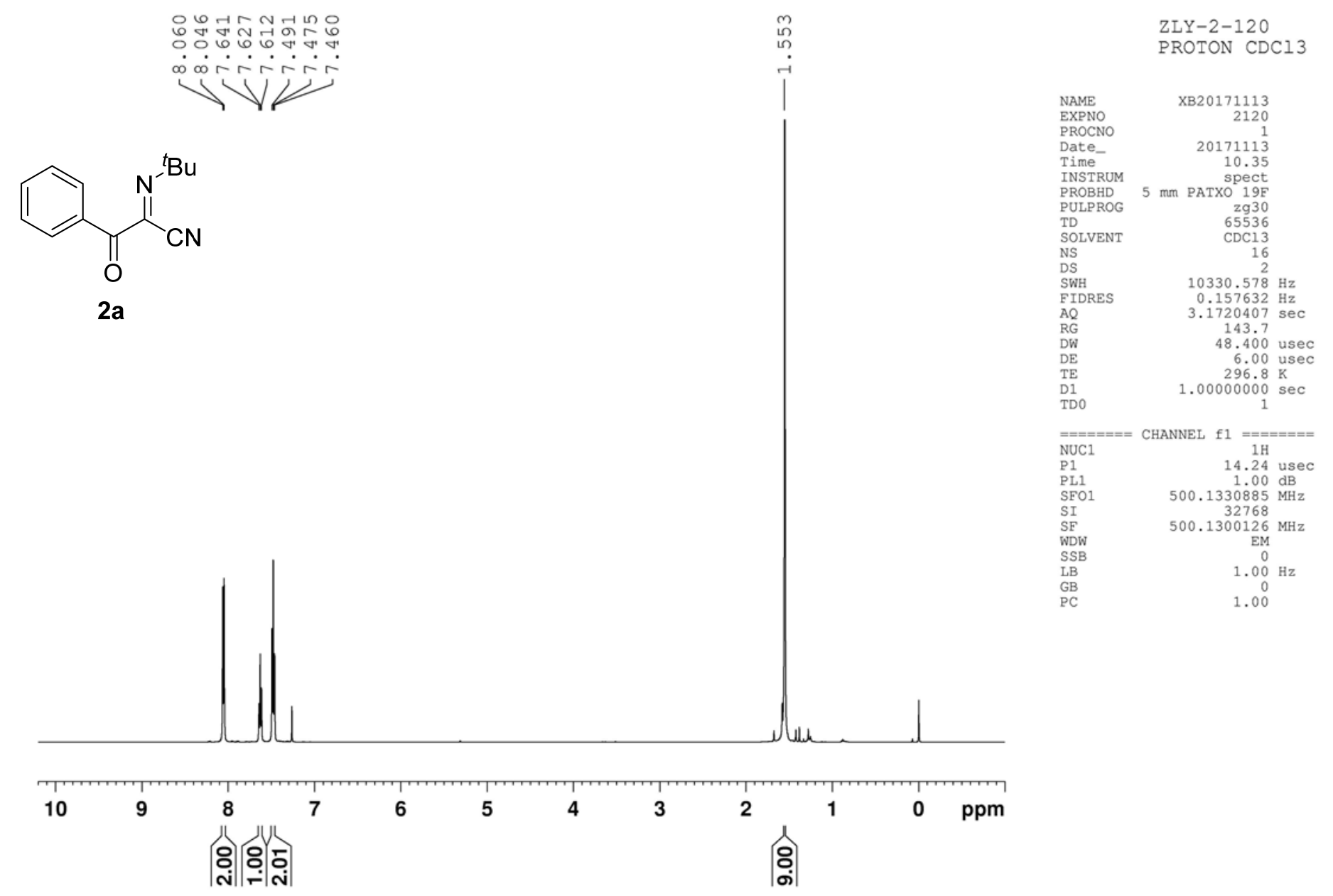




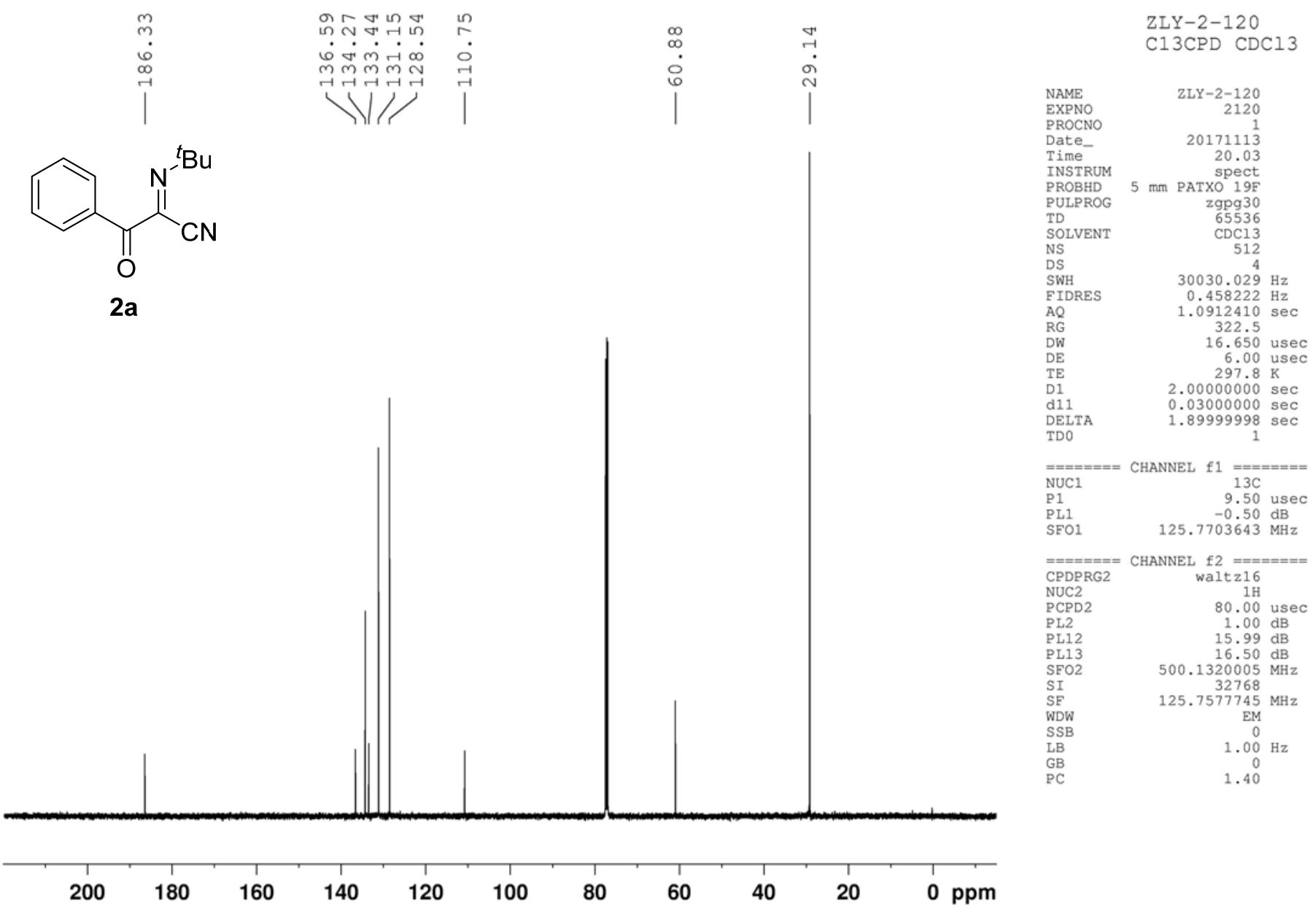




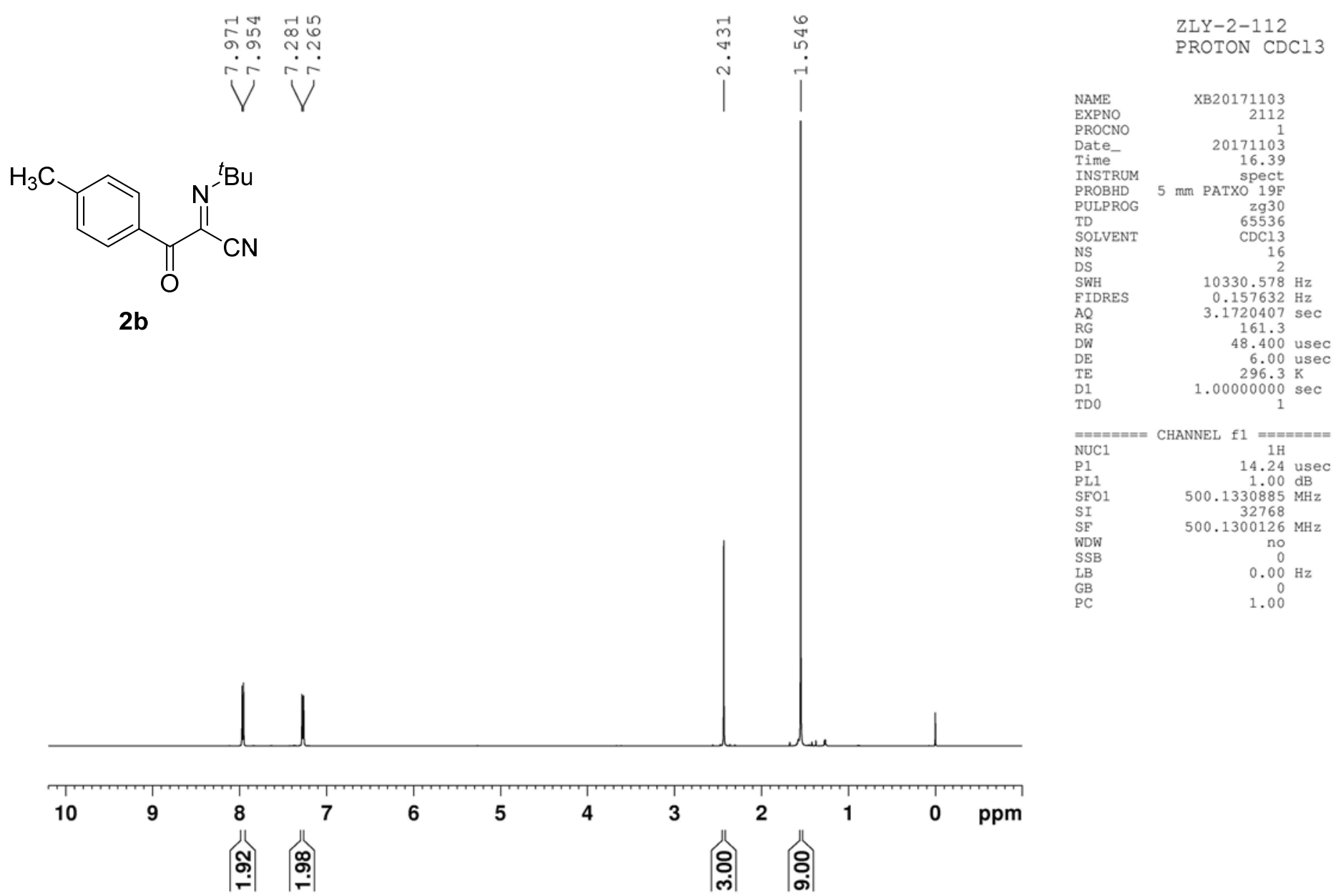




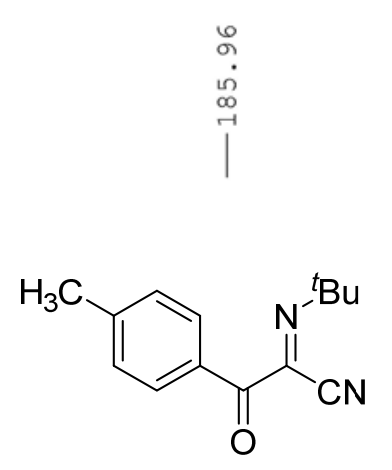

$2 b$

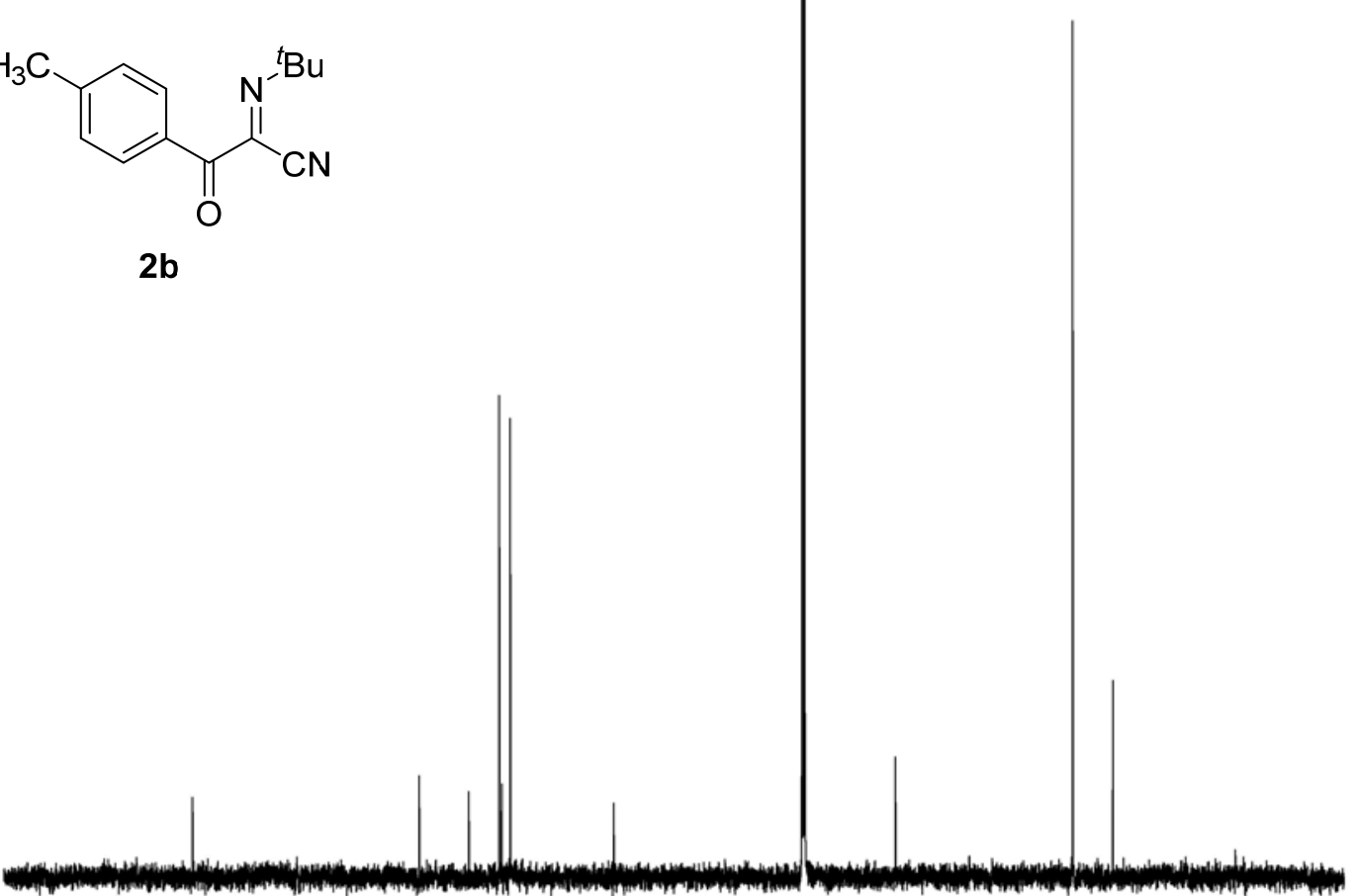

200

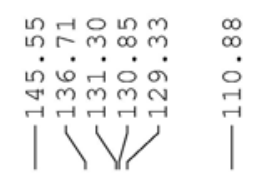

.

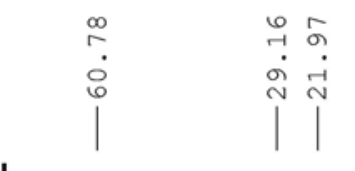

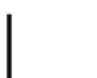

ZLY-2-112
C13CPD CDC13

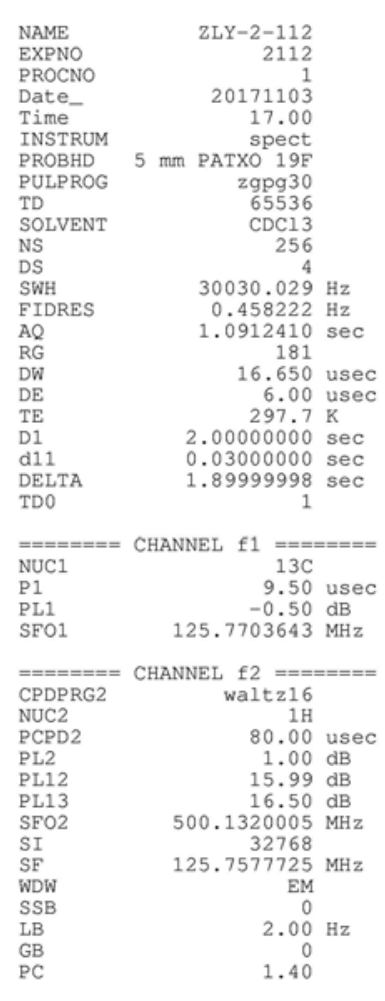




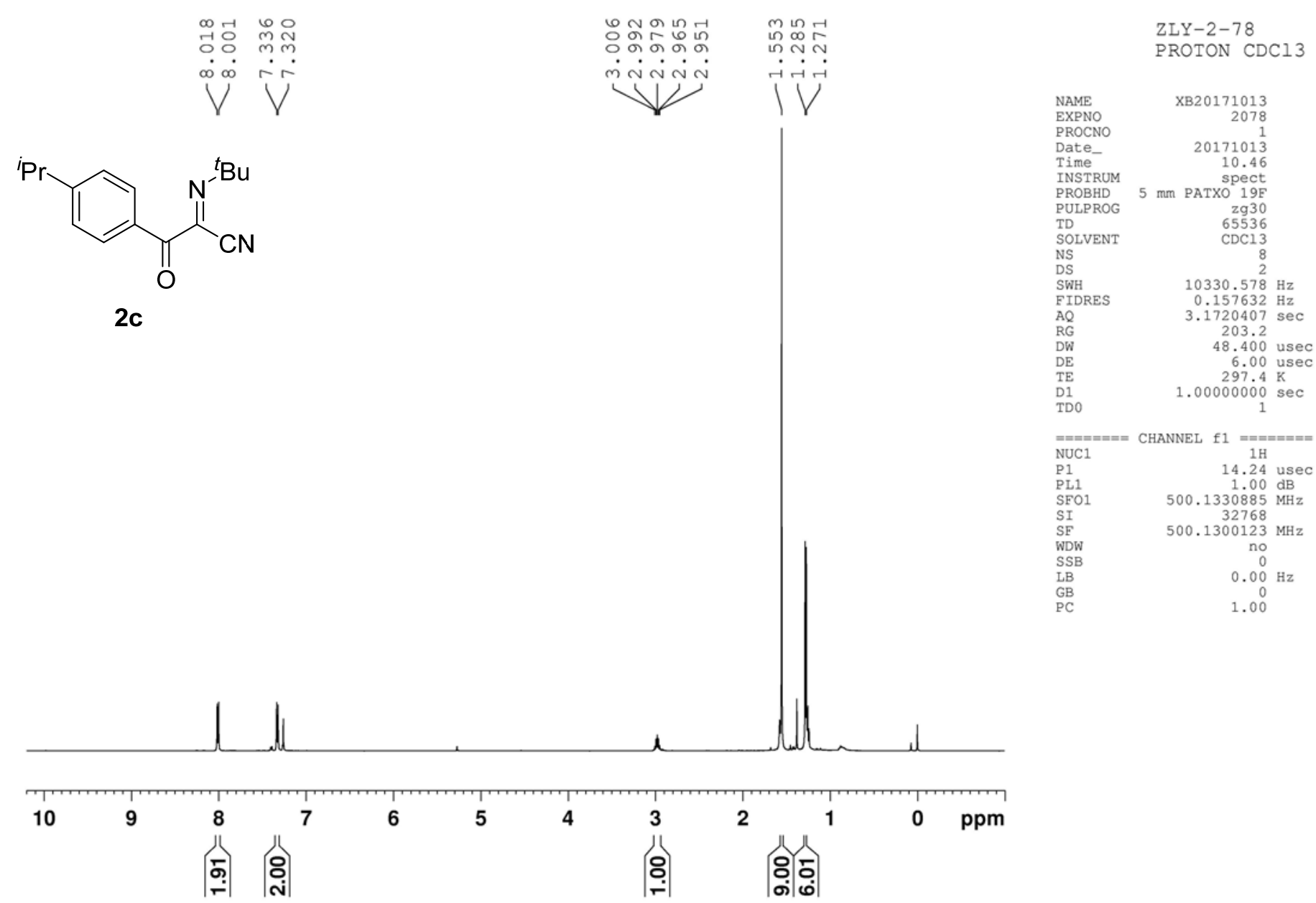




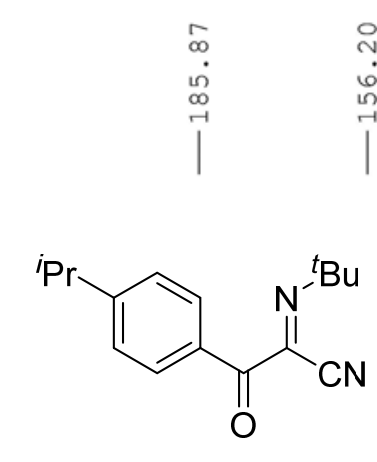

2c
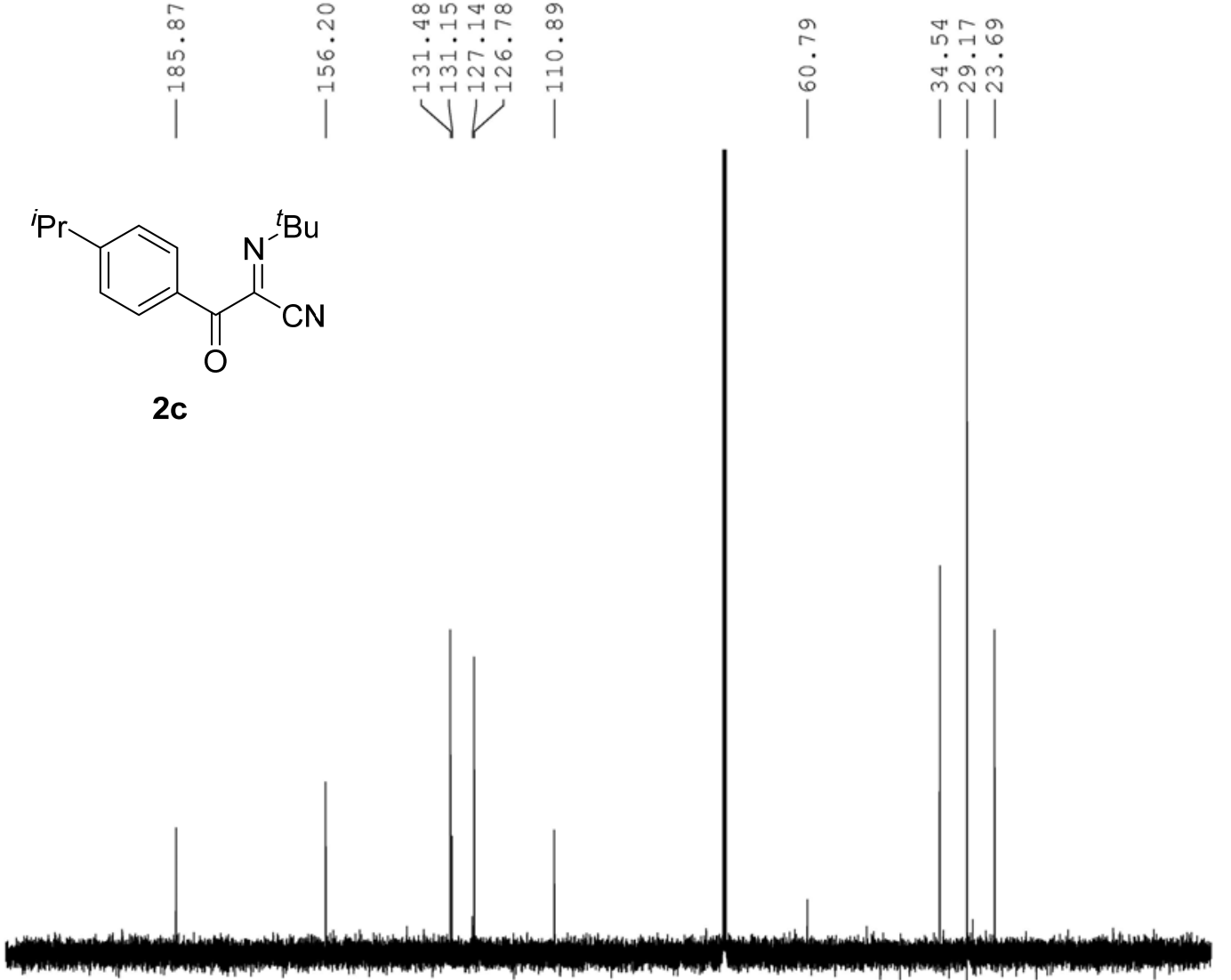

200
ZLY-2-78
C13CPD CDC13

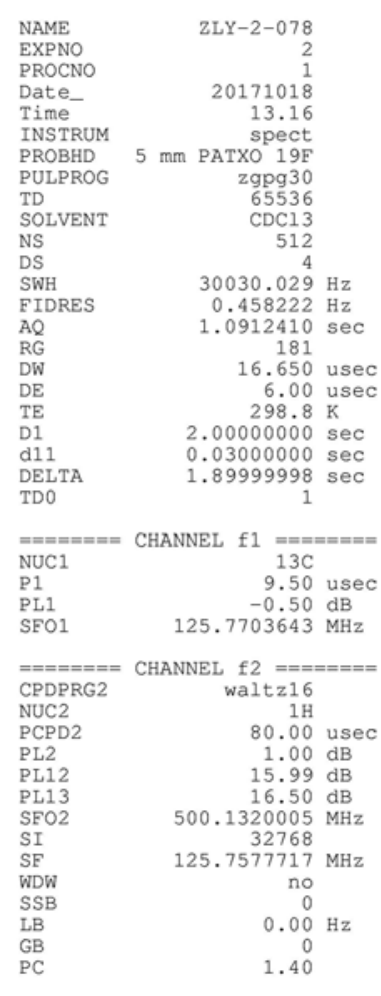



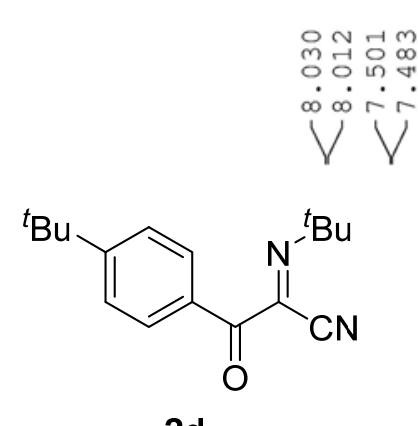

2d

$\mathrm{CN}$
品思

ii

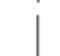

$\mid$

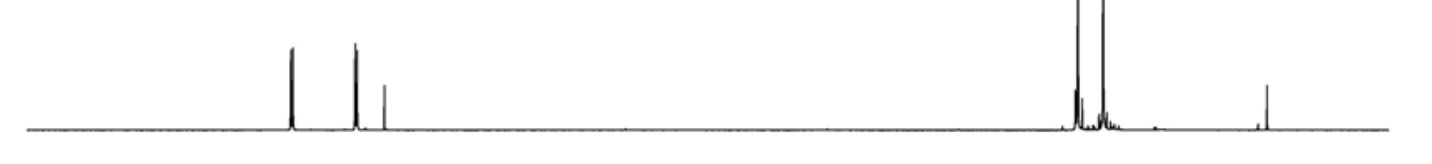

10
唡 勇
이으
이음 


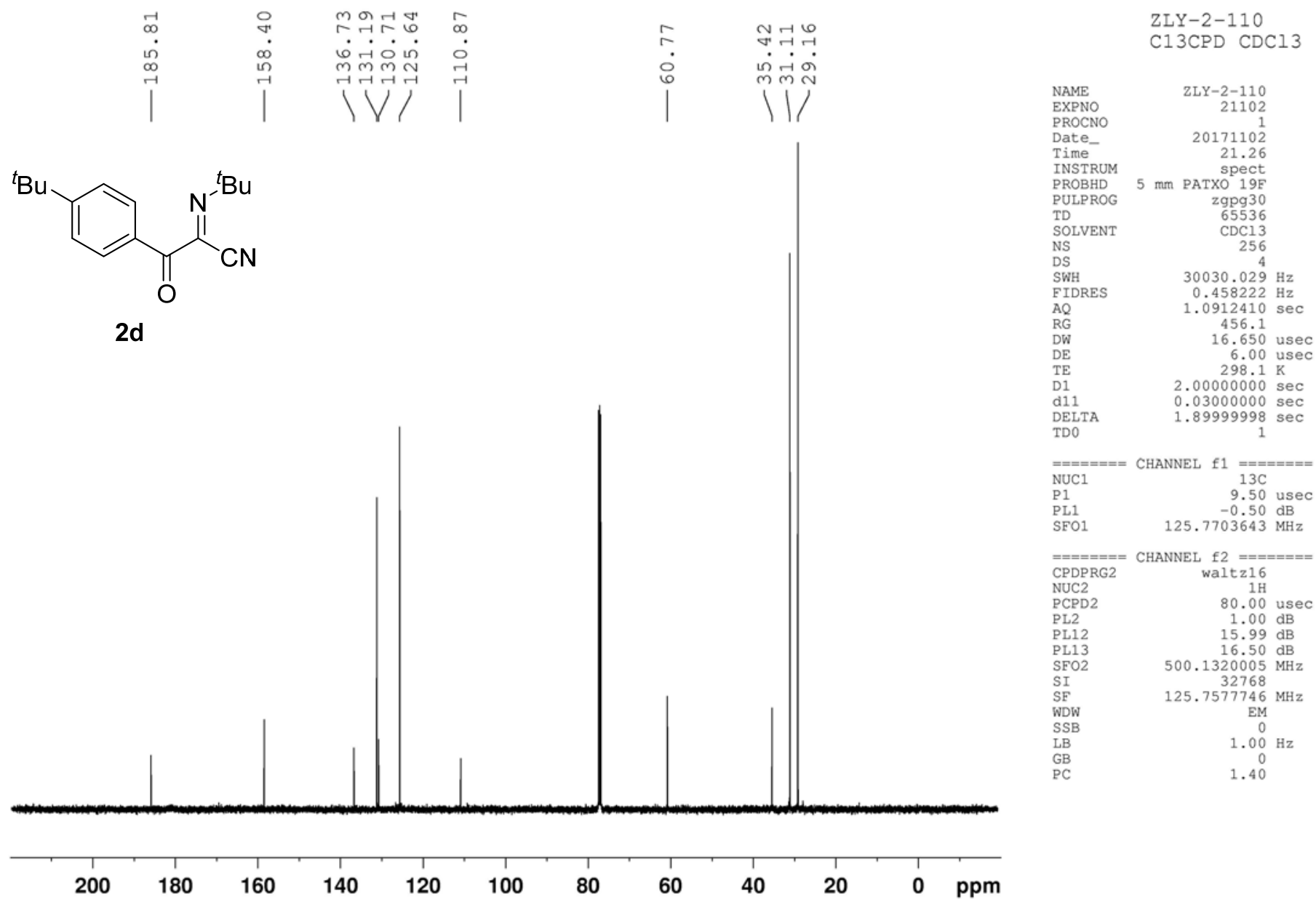




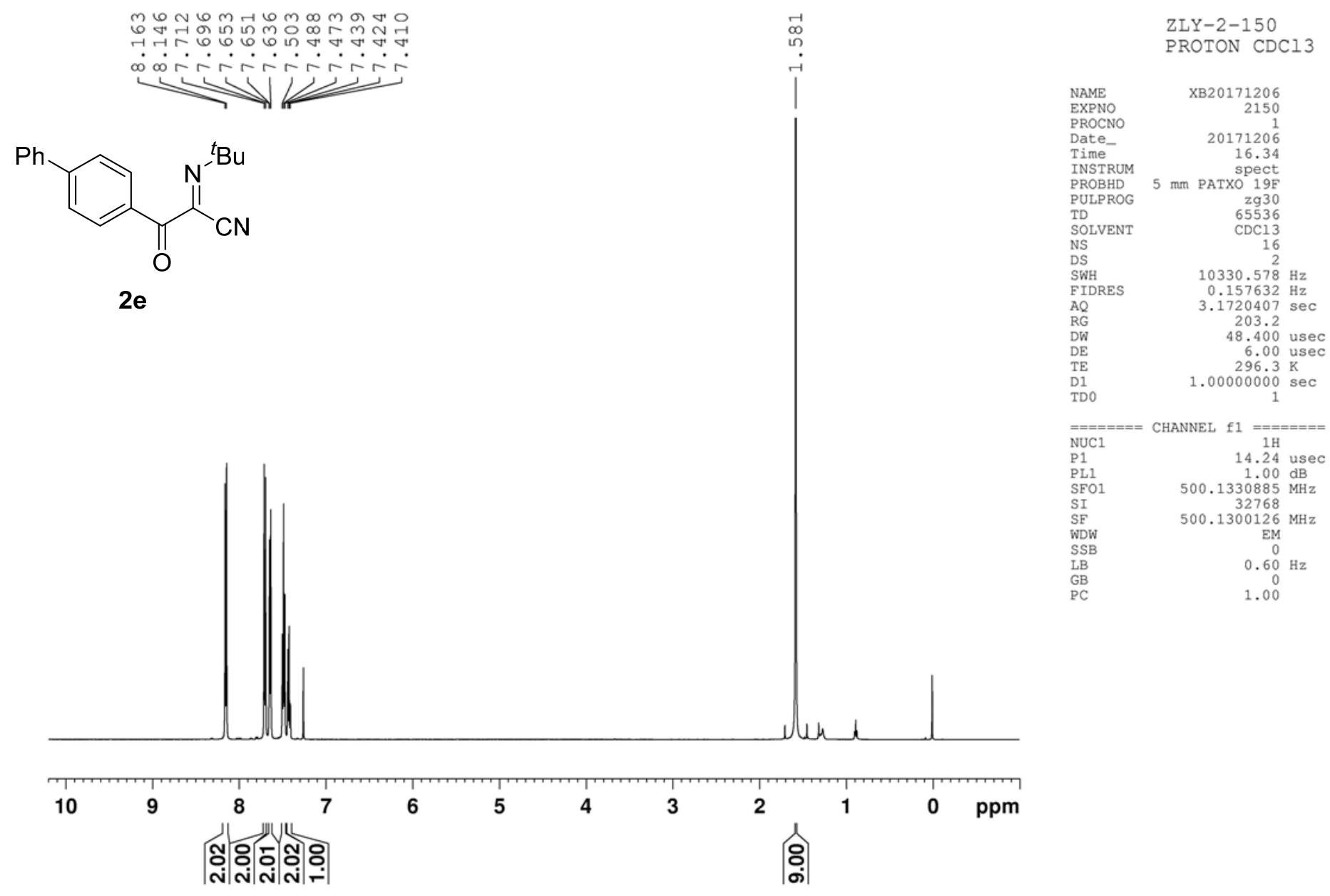




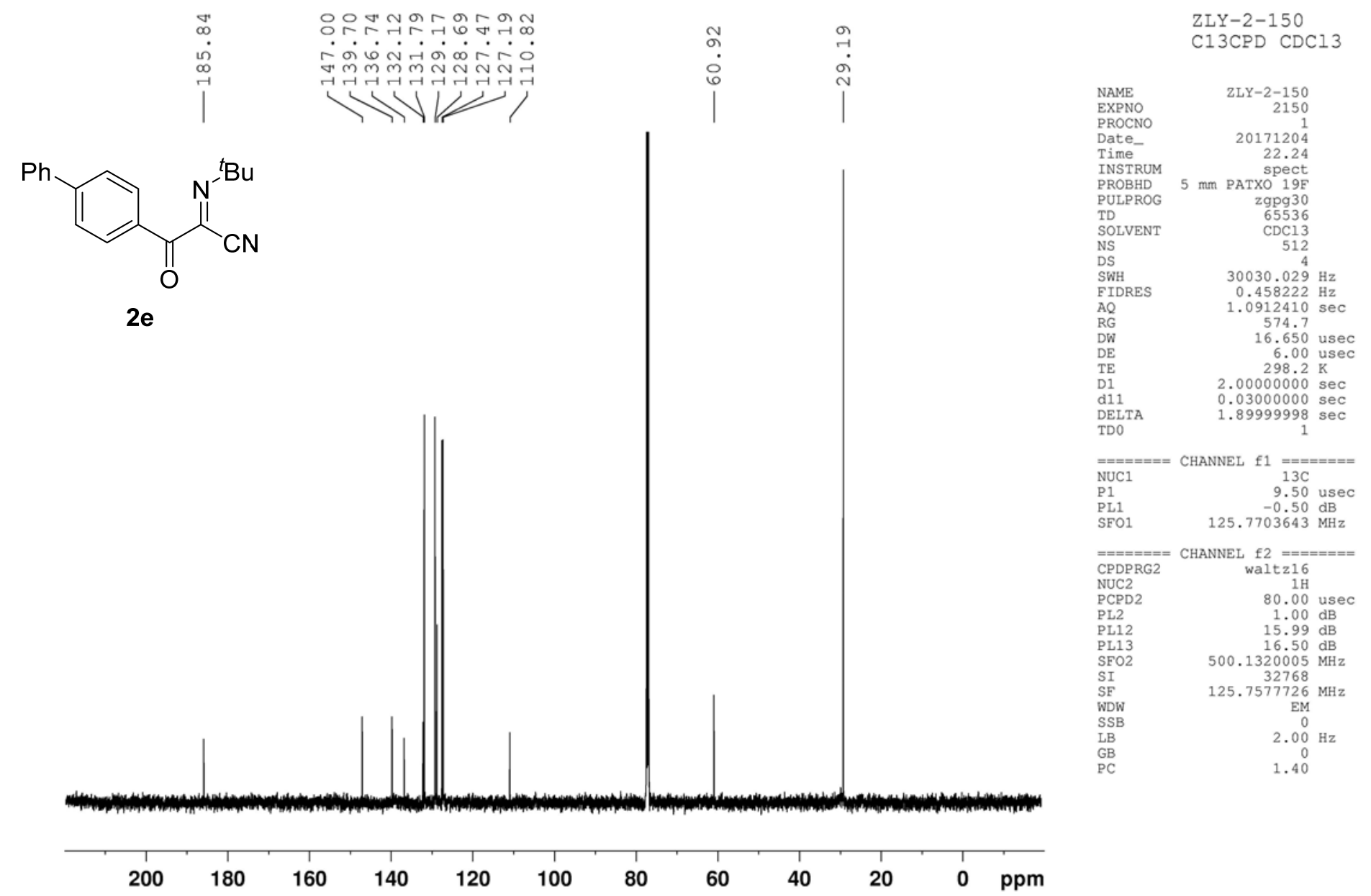




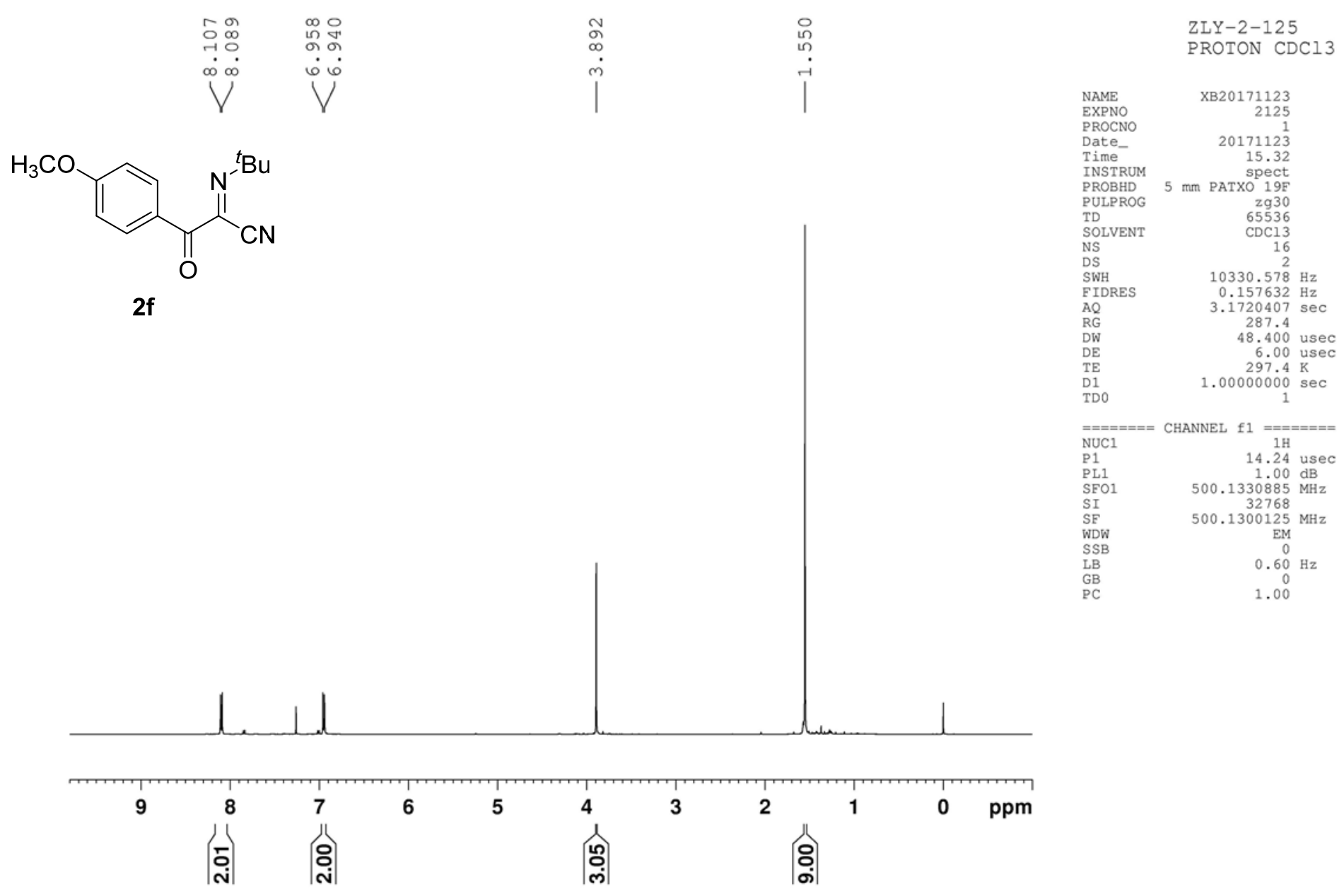



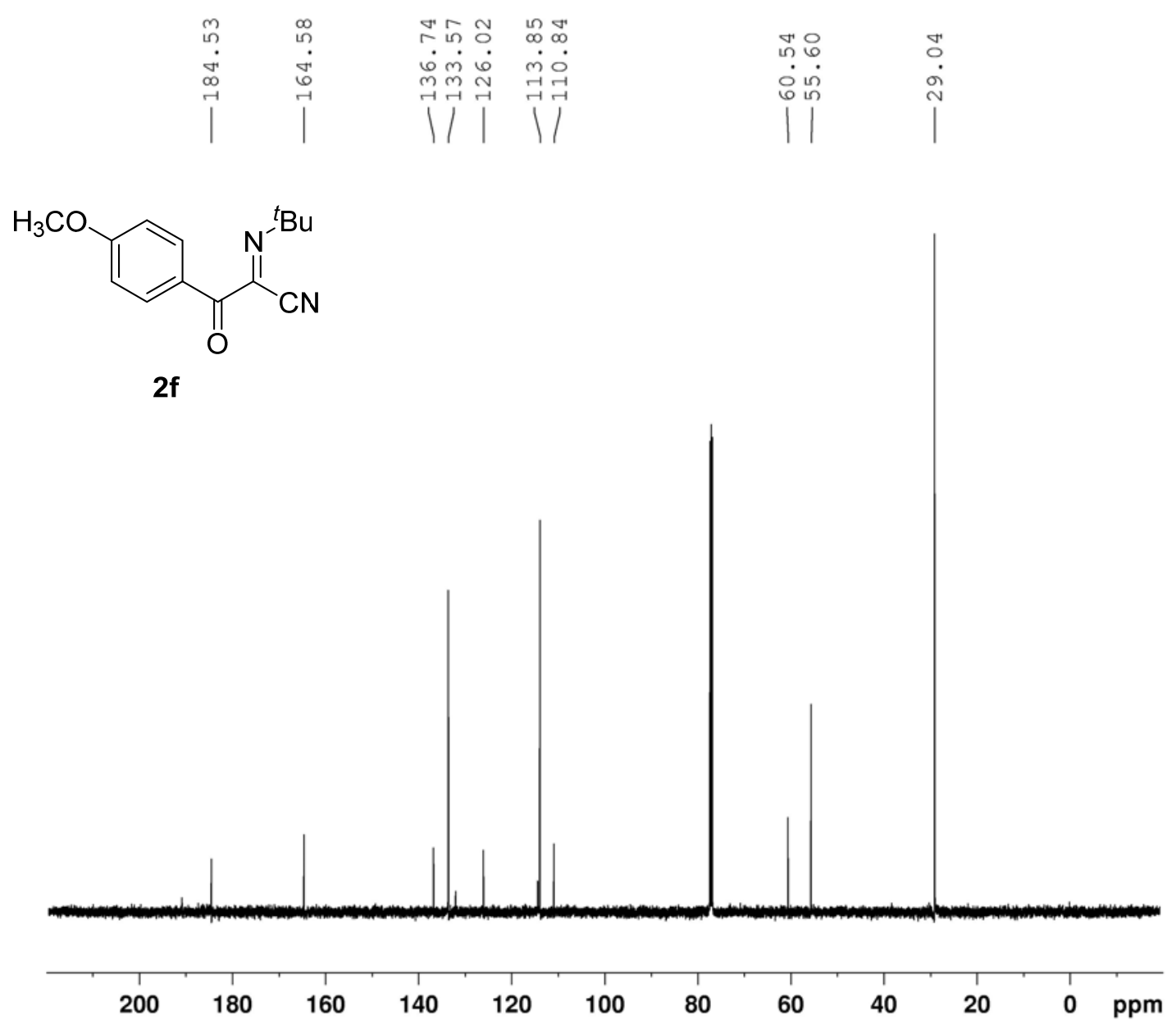

ZLY-2-125

C13CPD CDC13

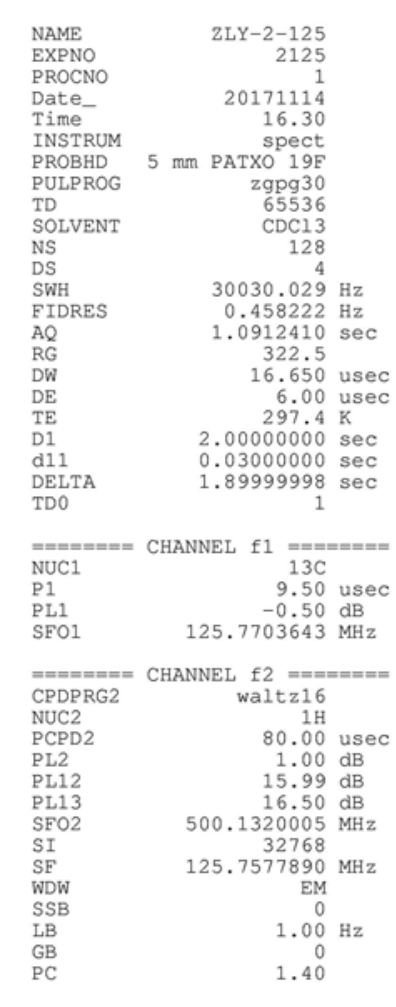




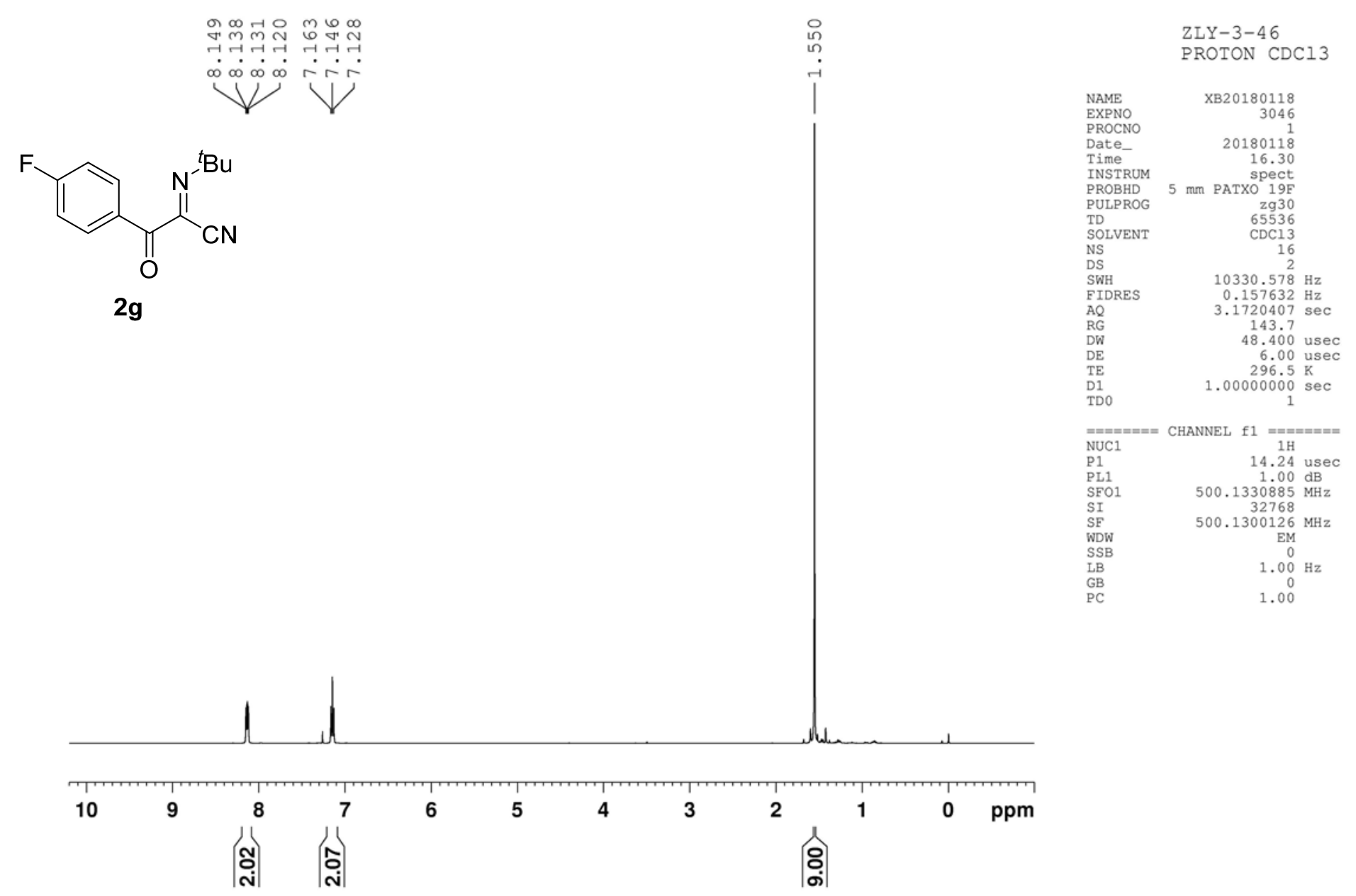



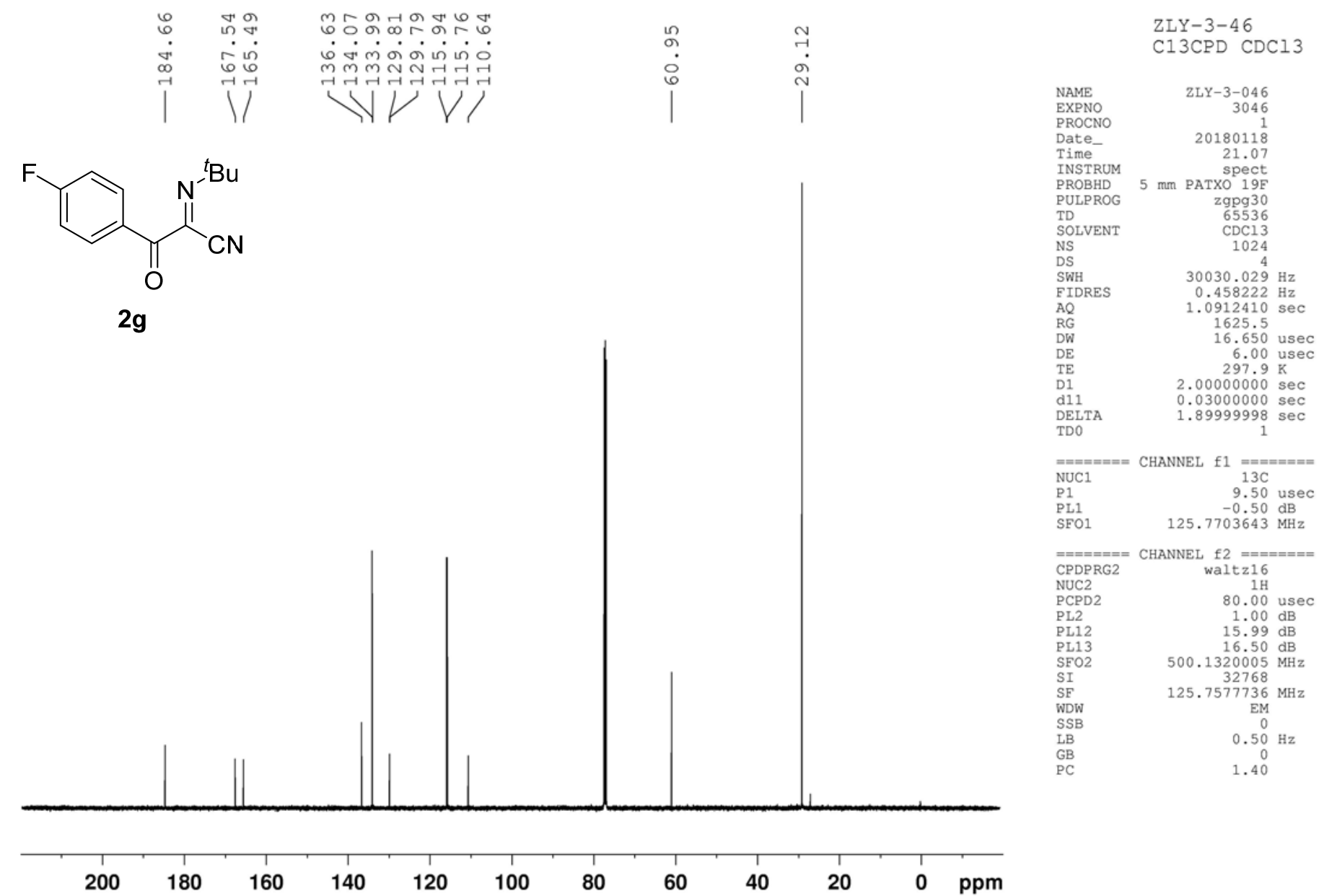


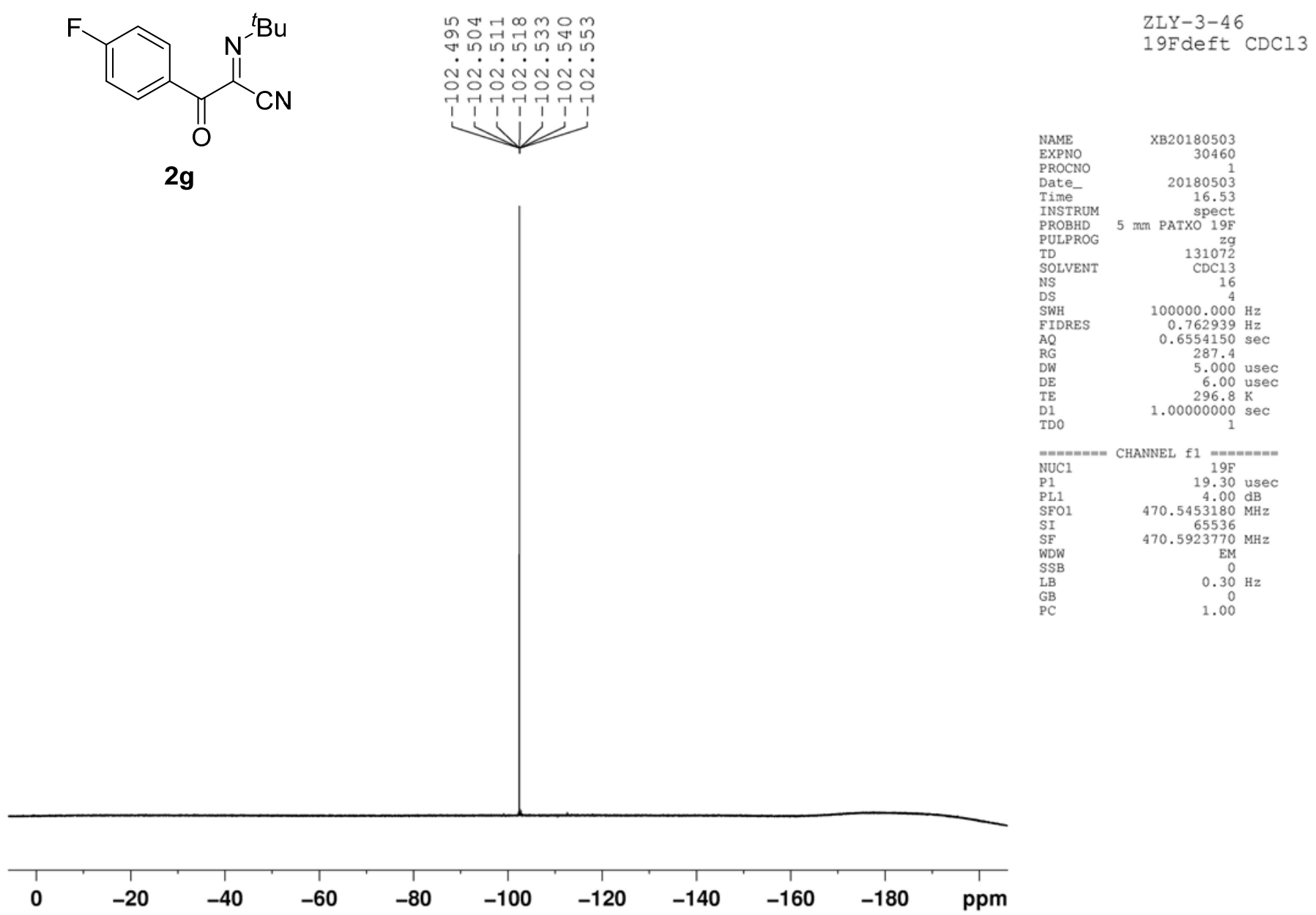




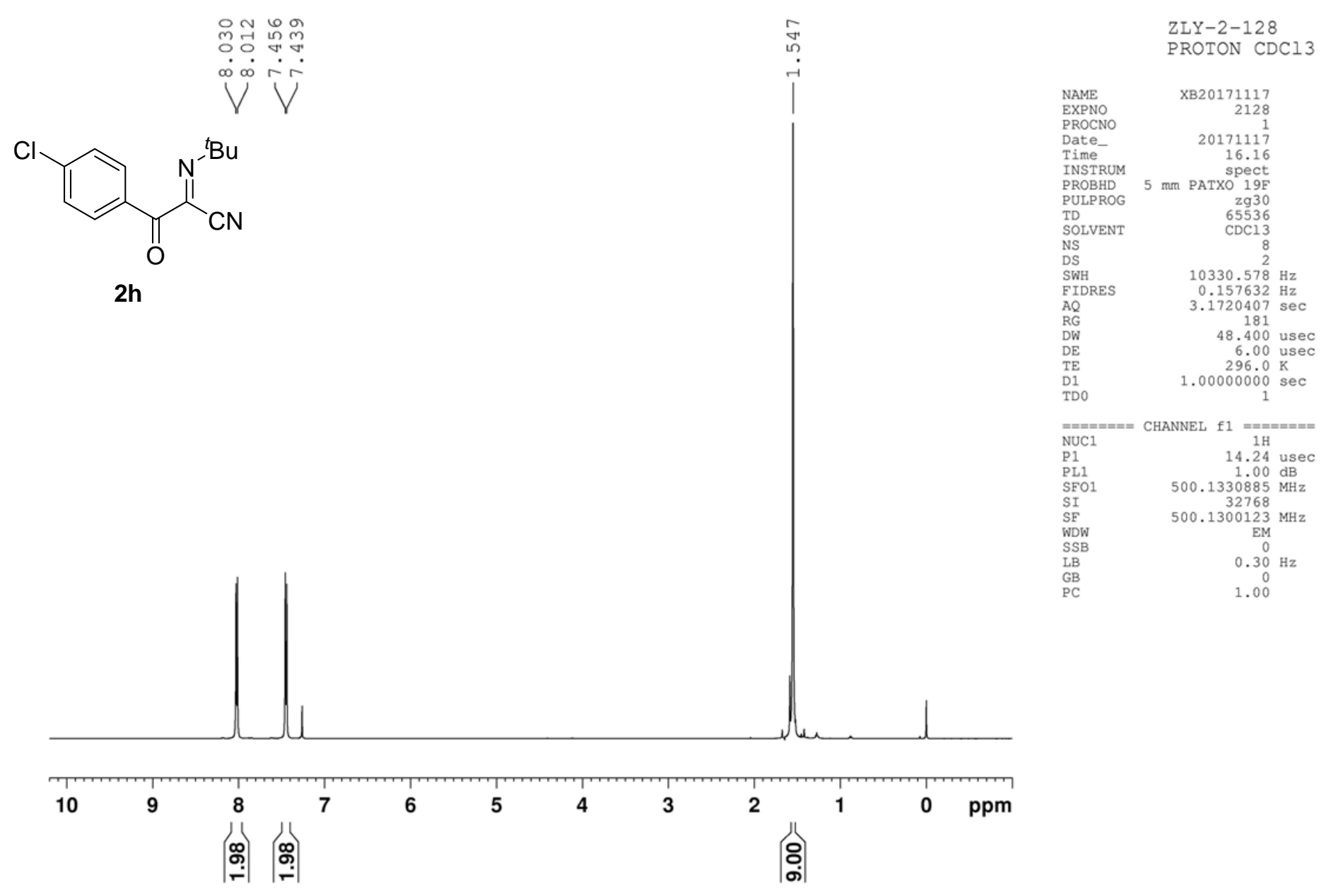




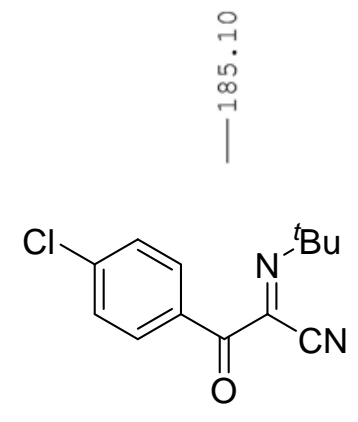

2h

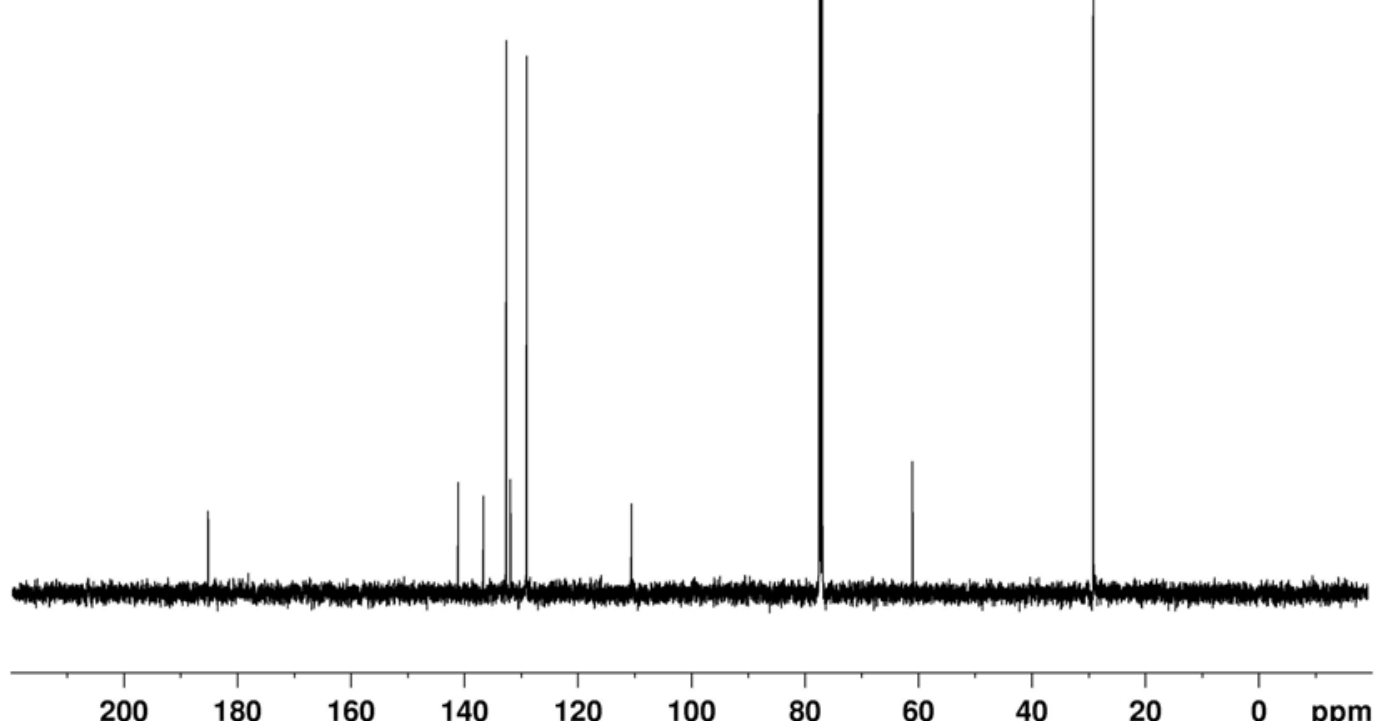

ZLY-2-128

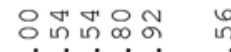

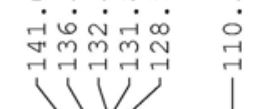

$\stackrel{N}{0}^{\stackrel{0}{0}}$

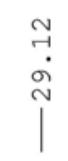

CPD CDC13

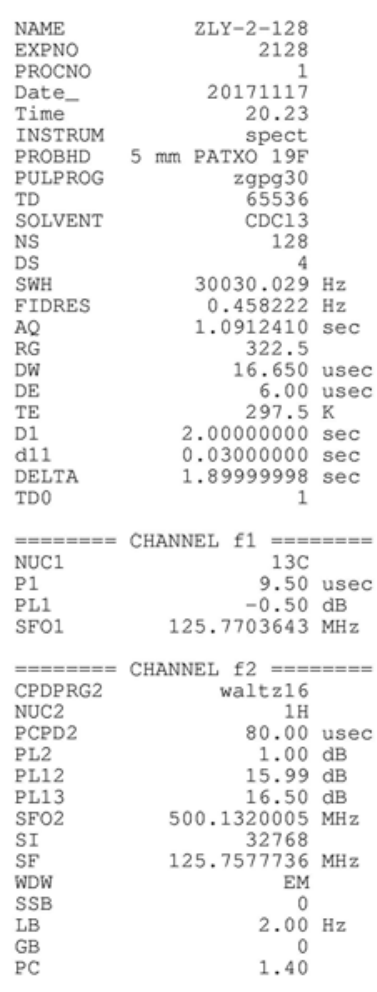




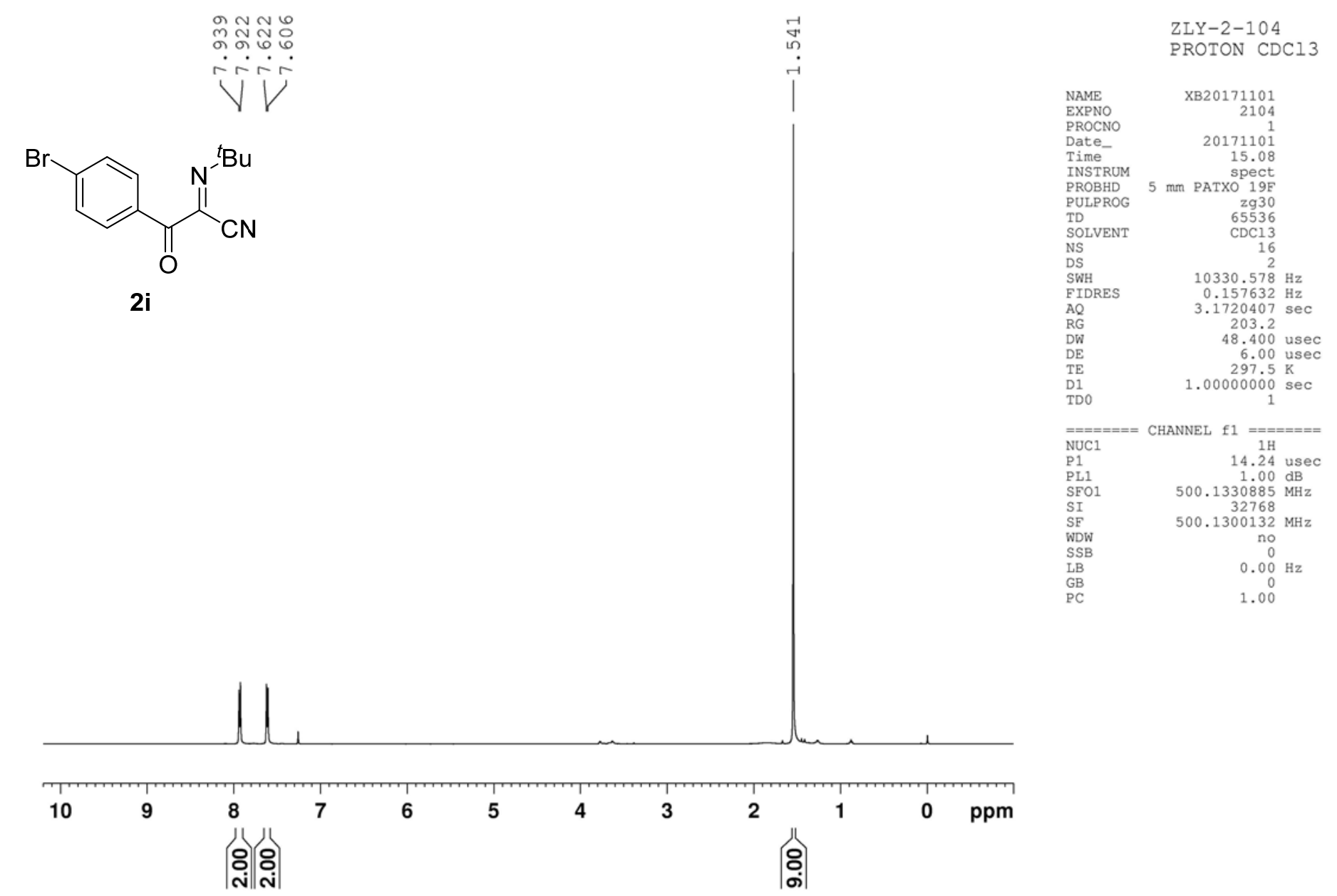




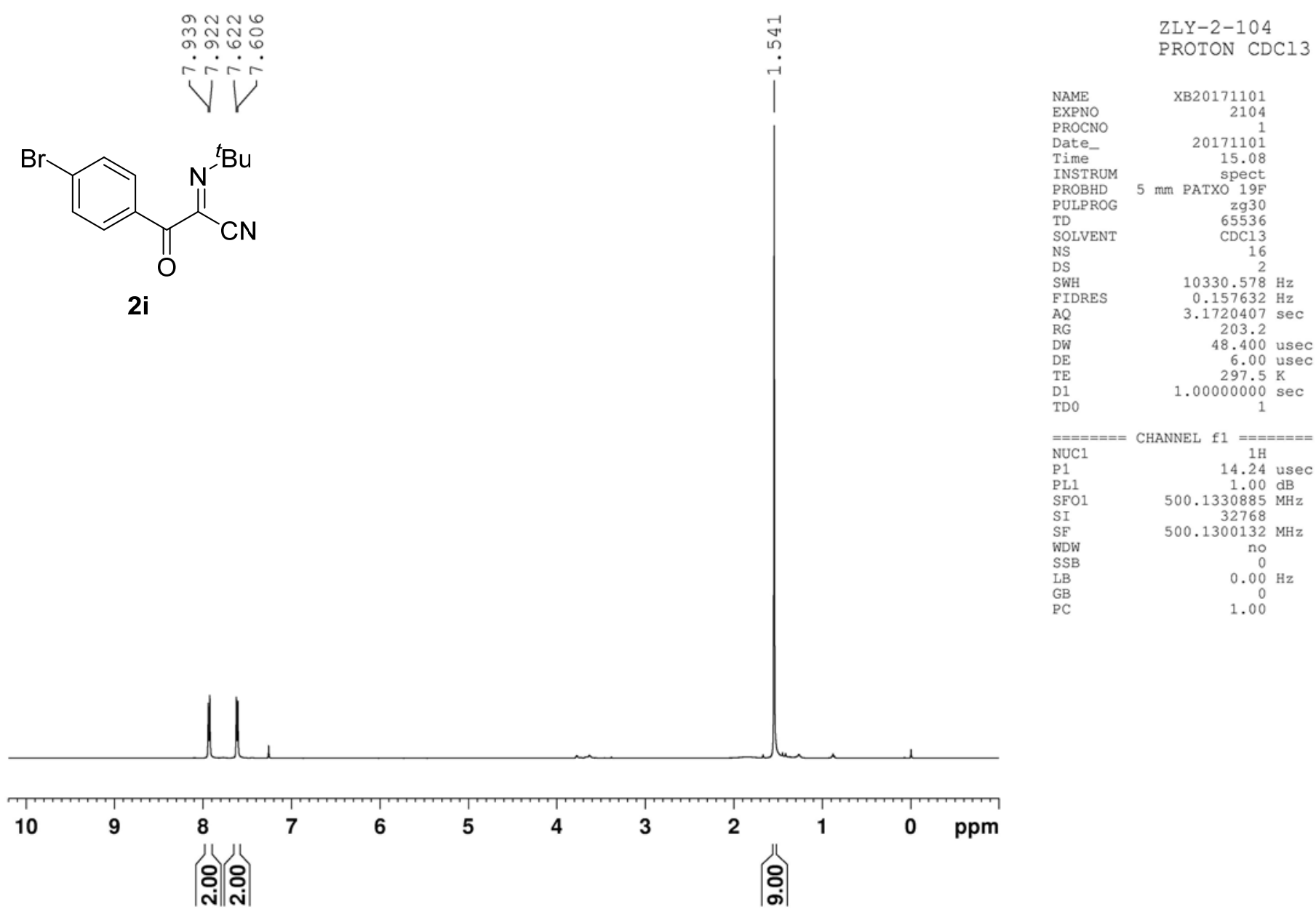




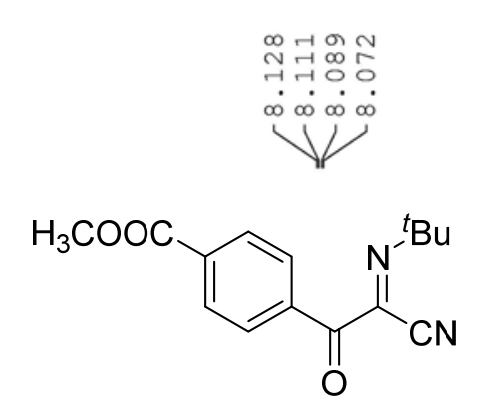

2j

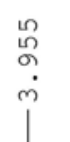

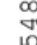
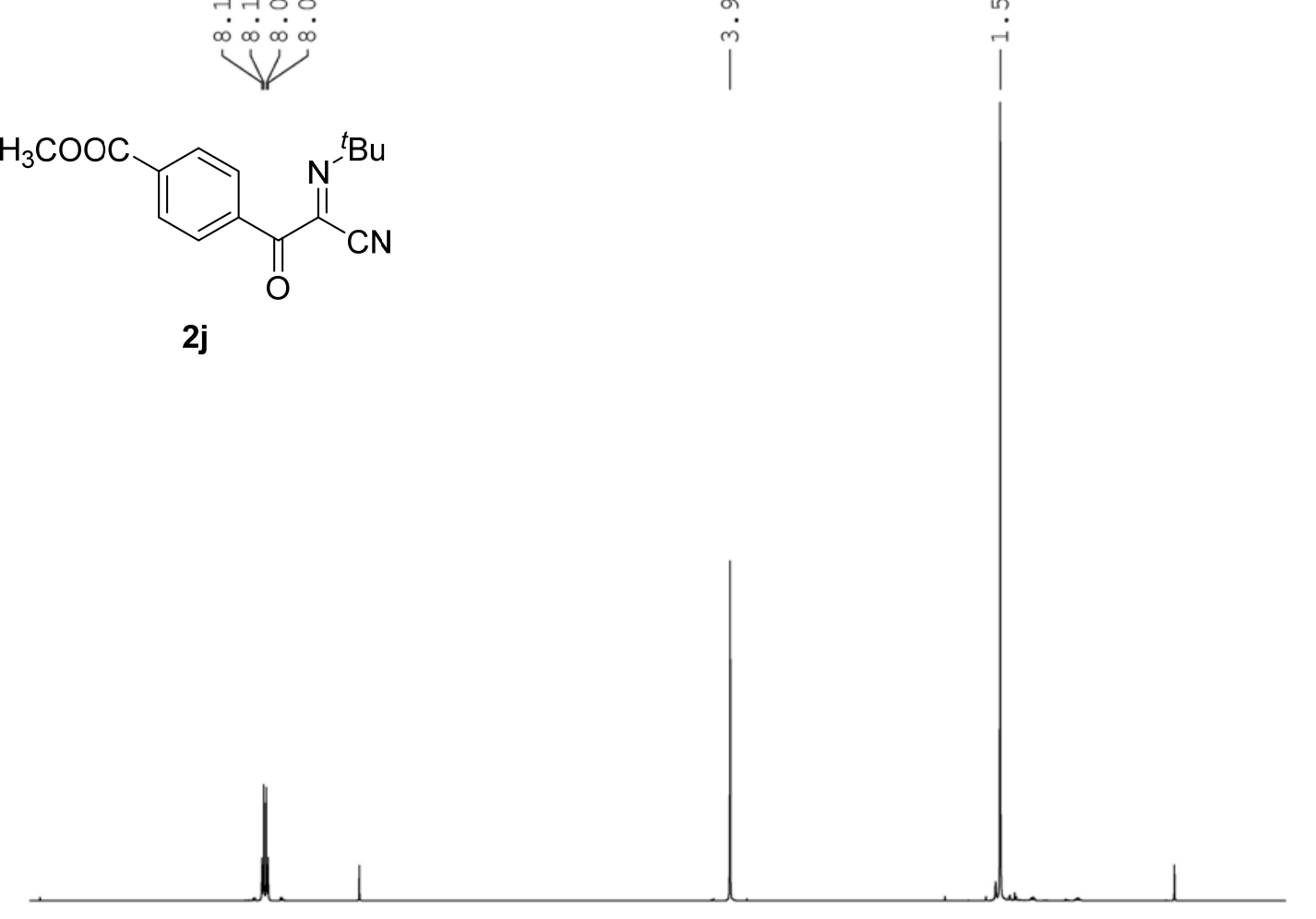

10

43

2 오옹 둘 命 


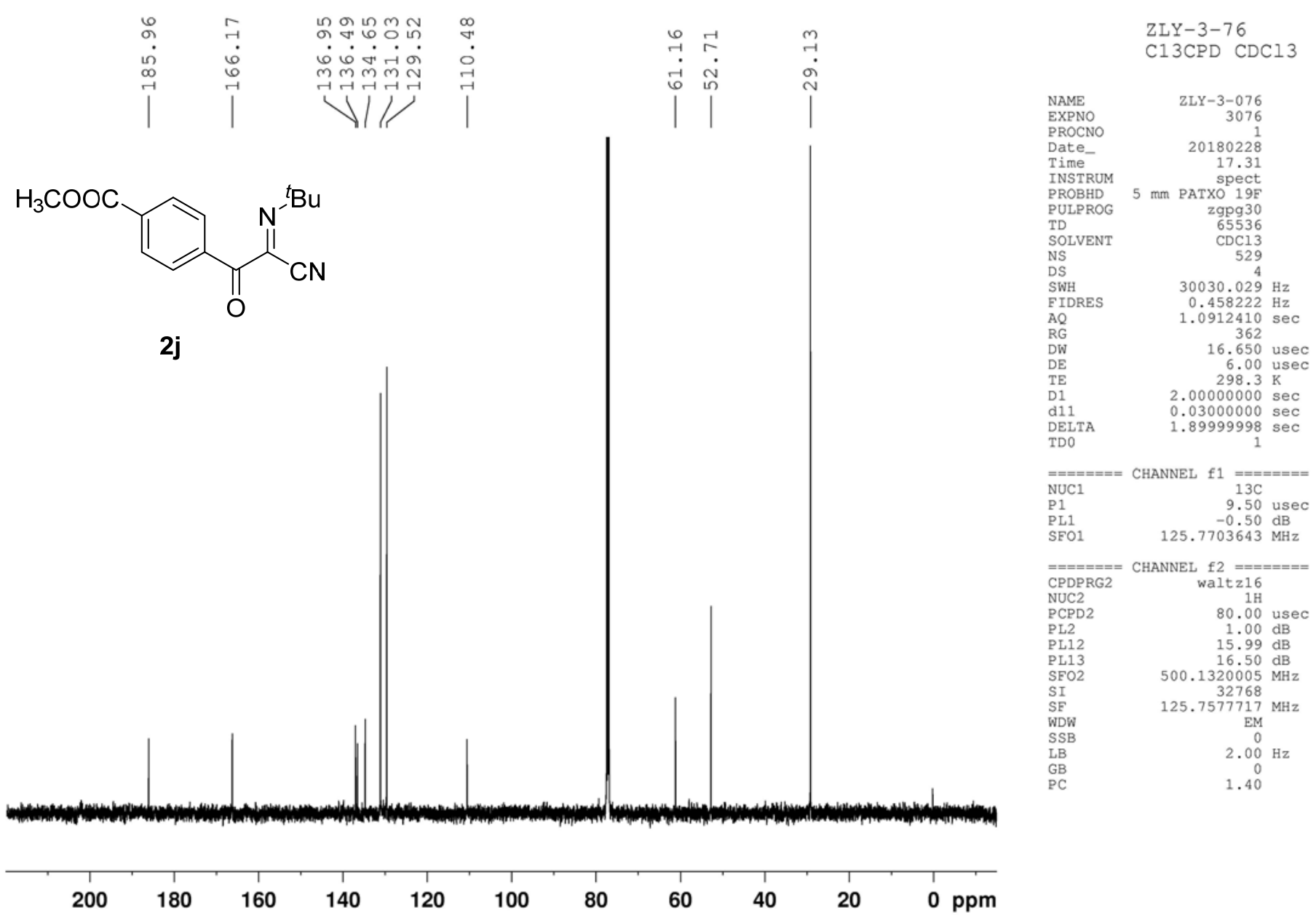




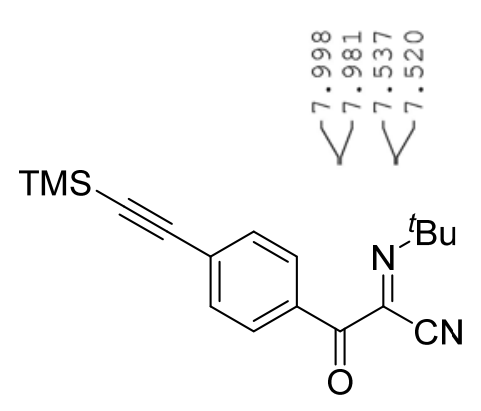

$2 k$

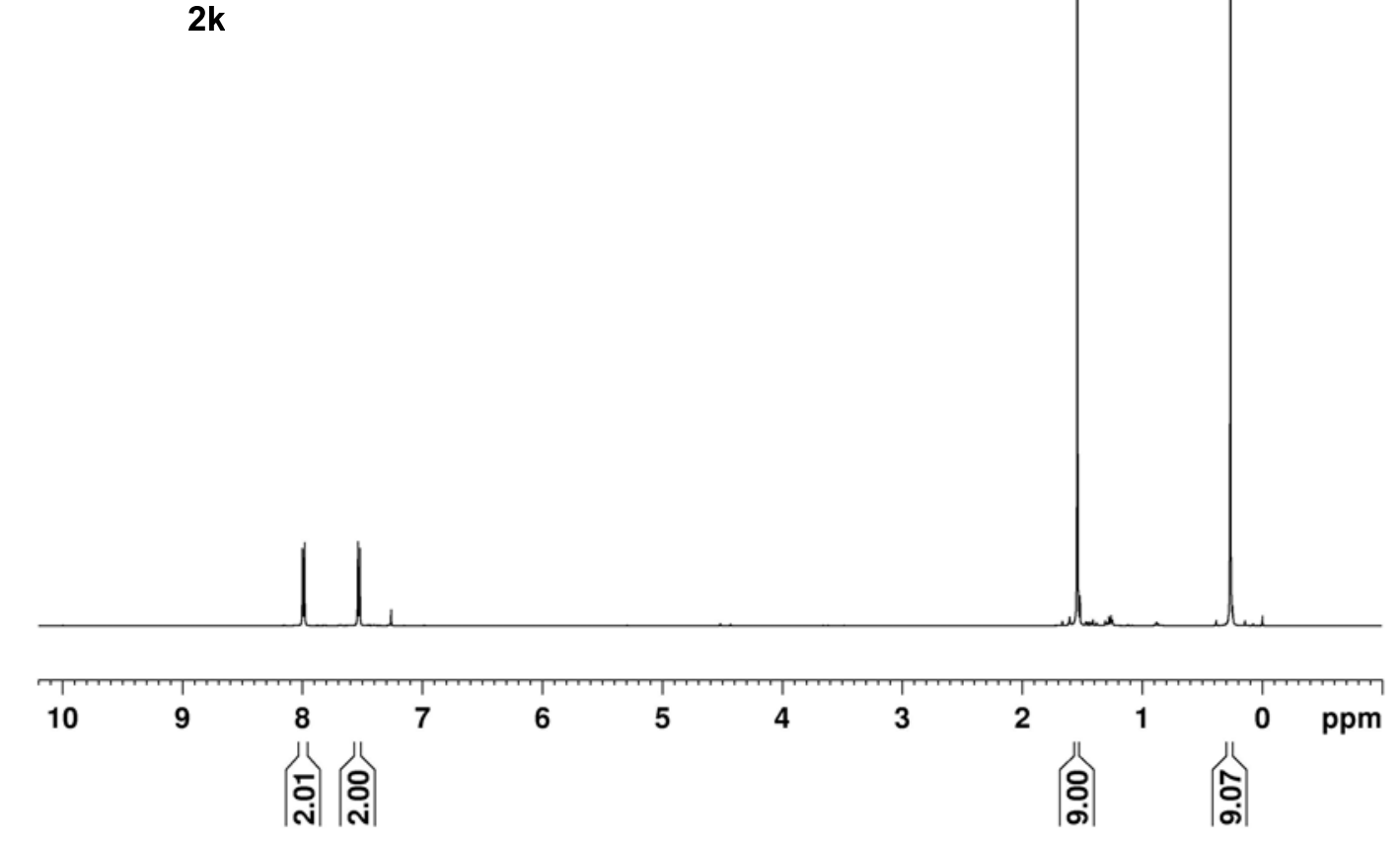

ZLY-2-154

PROTON CDC13

$$
\begin{aligned}
& \begin{array}{lr}
\text { NAME } & \text { XB20171211 } \\
\text { EXPNO } & 2154 \\
\text { PROCNO } & 20171211
\end{array} \\
& \begin{array}{lr}
\text { PROCNO } & 1 \\
\text { Date- } & 20171211 \\
\text { Time } & 10.20
\end{array} \\
& \begin{array}{c}
\text { IIme } \\
\text { IISTRU } \\
\text { PROBHD }
\end{array} \\
& \begin{array}{lr}
\text { PULPROG } & \text { mim PATXO } 19 \\
\text { TD } & 65536 \\
\text { SOLVENT } & 6536
\end{array} \\
& \begin{array}{l}
\text { SOLVENT } \\
\text { NS }
\end{array} \\
& \begin{array}{ll} 
& 10330.578 \mathrm{~Hz} \\
\text { FIDRES } & 0.157632 \mathrm{~Hz} \\
\text { AQ } & 3.1720407 \mathrm{sec}
\end{array} \\
& \begin{array}{lr}
\text { AQ } & 3.1720407 \mathrm{sec} \\
\text { RG } & 68.400 \text { usec } \\
\text { DW } & 4.00
\end{array} \\
& \begin{array}{lr}
29.00 \mathrm{~K} \\
\text { TE } & 1.00000000 \mathrm{Kec}
\end{array} \\
& \begin{array}{lr}
=======\text { CHANNEL } \mathrm{f} 1 \mathrm{1}======= \\
\text { NUC1 } & 1 \mathrm{H} \\
\text { P1 } & 14.24 \mathrm{usec} \\
\text { PL1 } & 1.00 \mathrm{~dB} \\
\text { SFO1 } & 500.1330885 \mathrm{MHz} \\
\text { SI } & 32768 \\
\text { SF } & 500.13000 \mathrm{MHz} \\
\text { WDW } & \mathrm{EM} \\
\text { SSB } & 0 \\
\text { LB } & 0.50 \mathrm{~Hz} \\
\text { GB } & 0 \\
\text { PC } & 1.00
\end{array}
\end{aligned}
$$

ต 


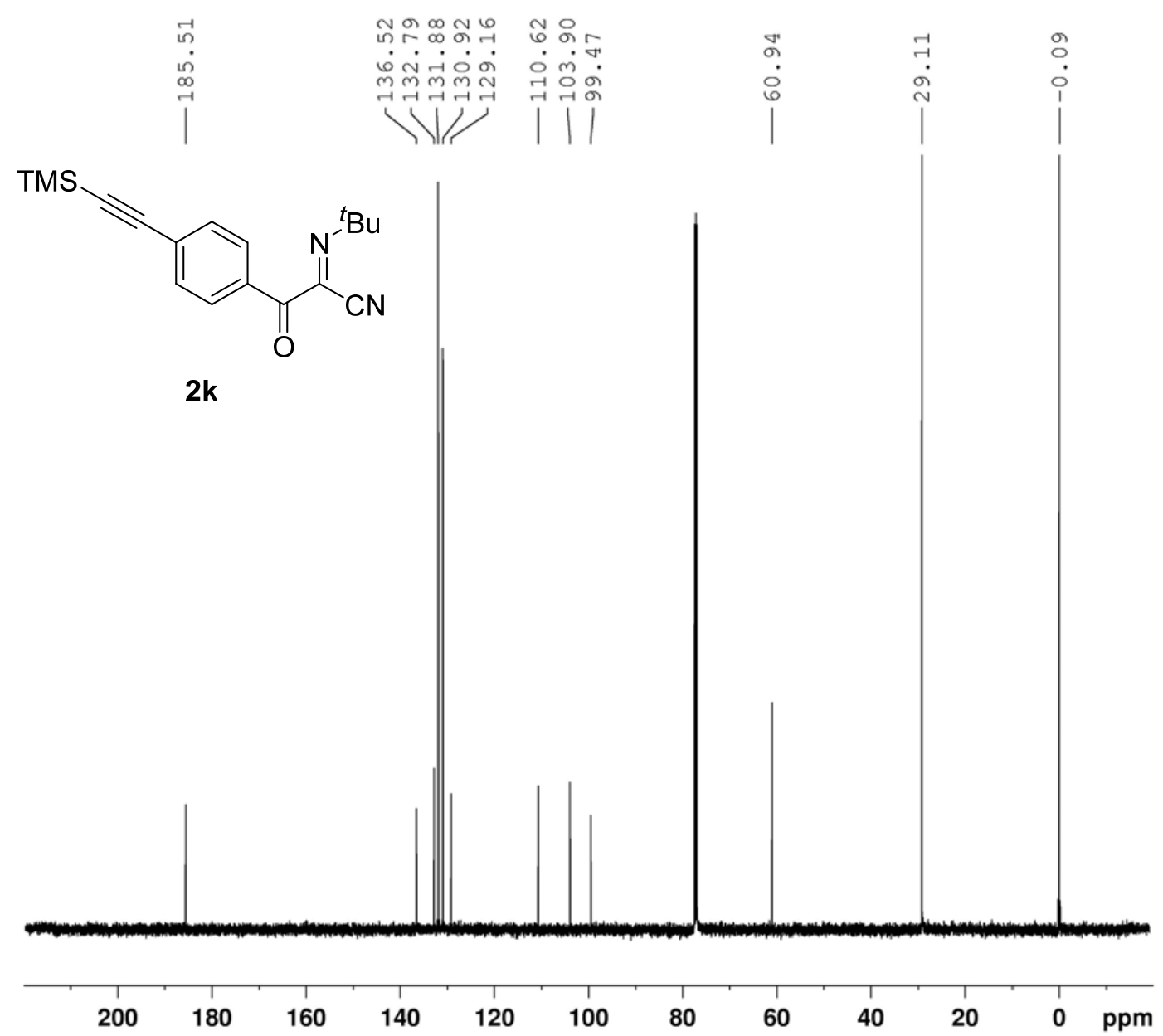

ZLY-2-154

C13CPD CDC13

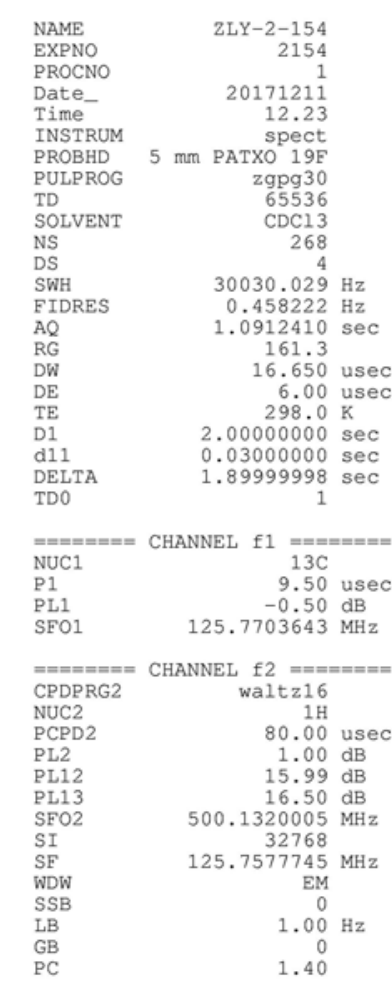

200 


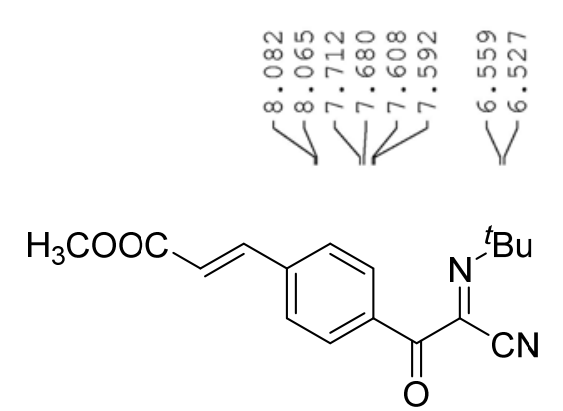

21 $\underset{\substack{m \\ \infty}}{\stackrel{m}{m}}$
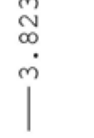

品

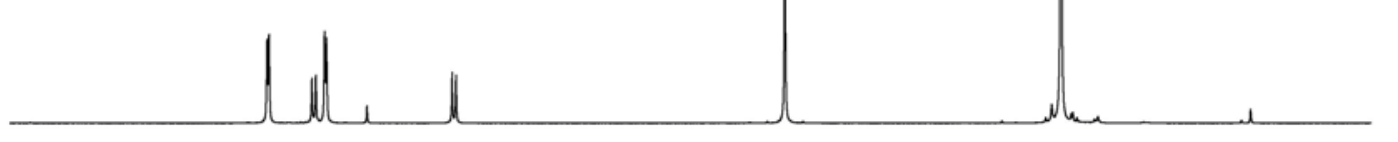

7

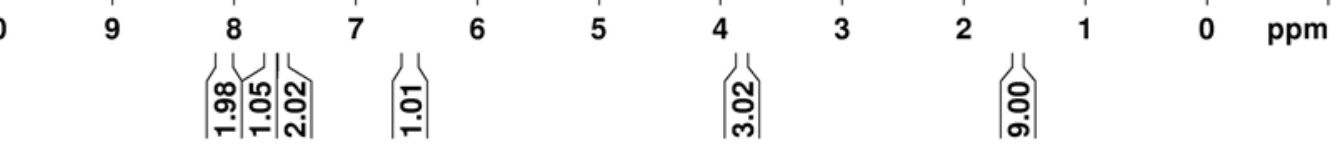




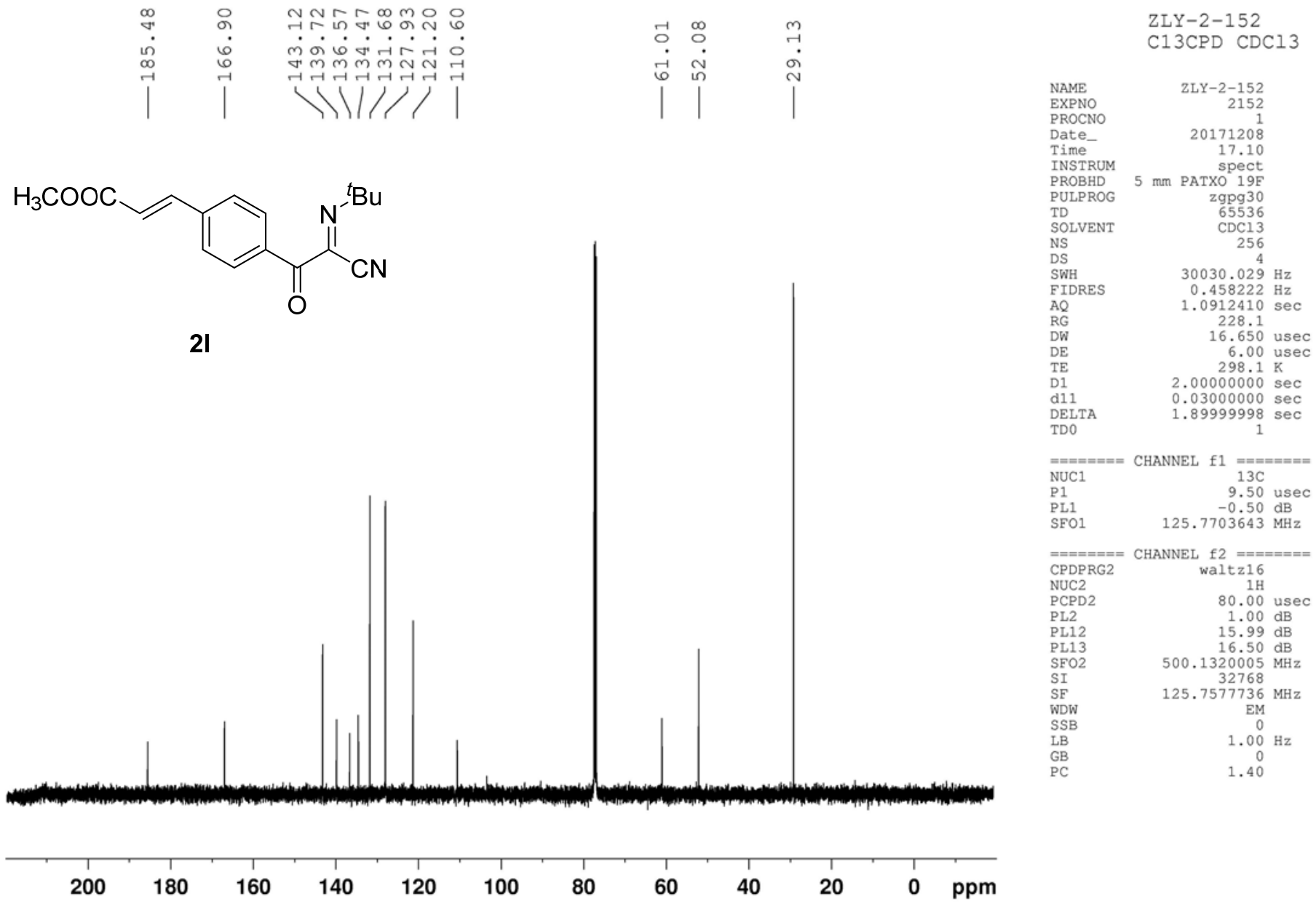




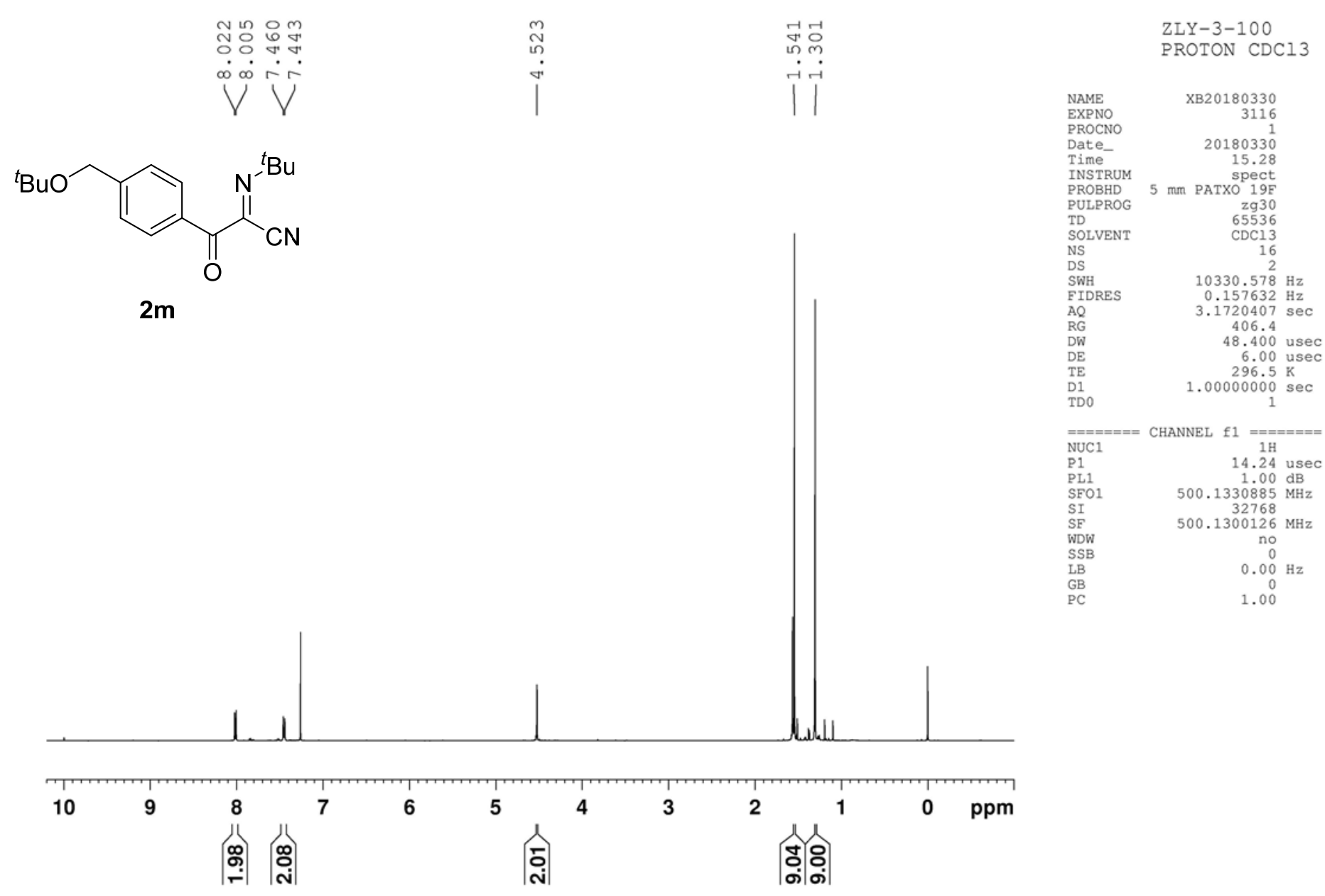




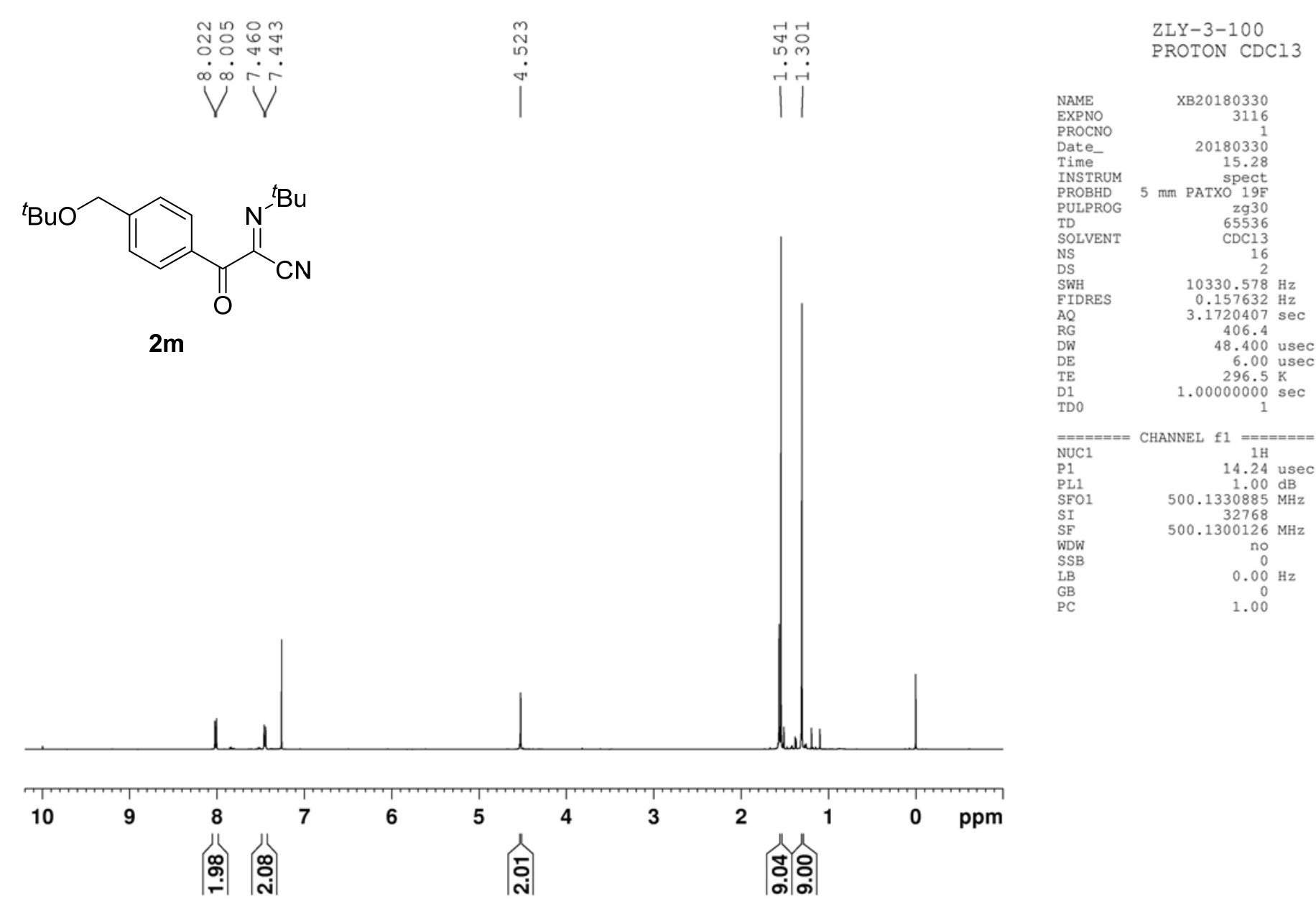




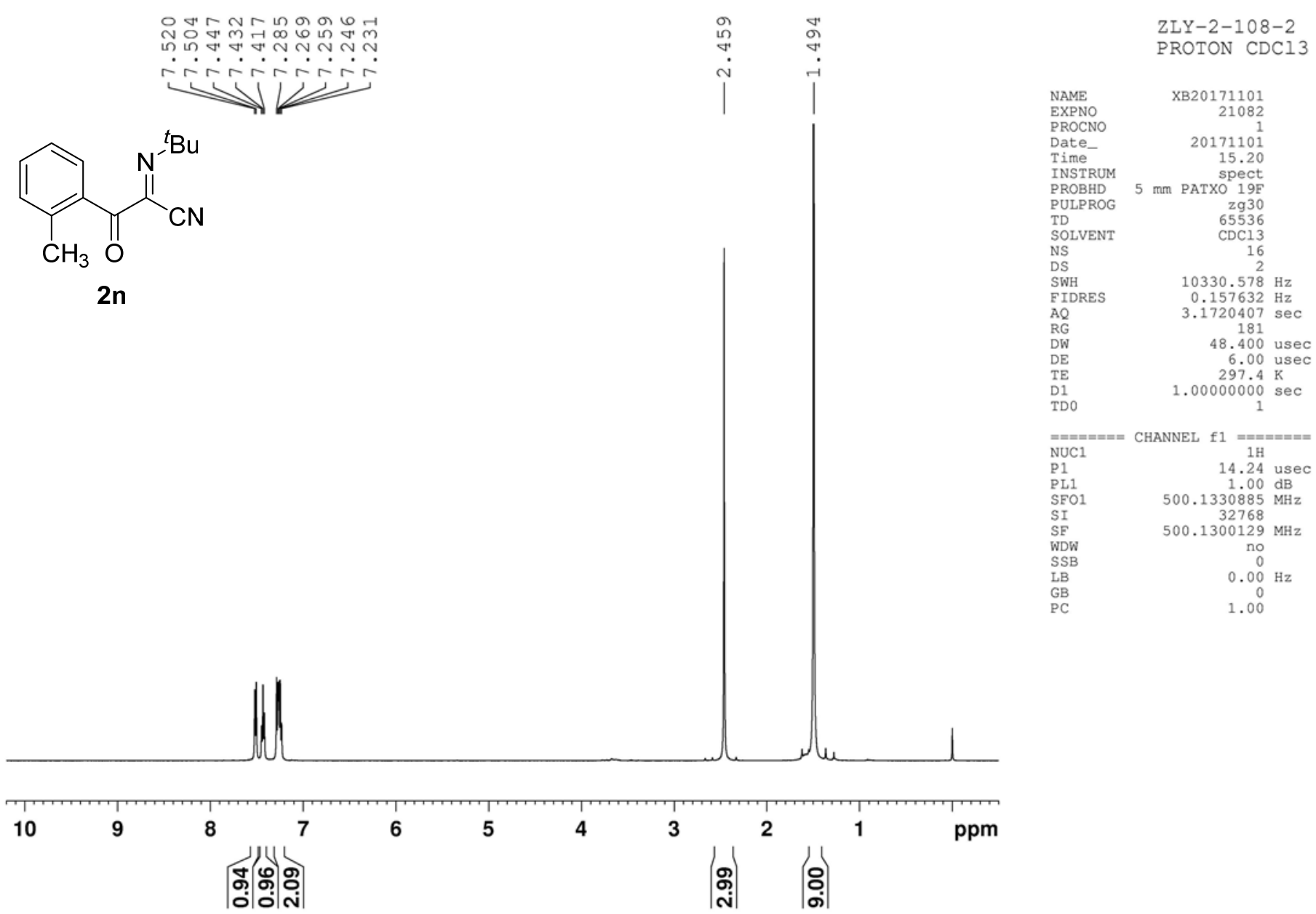




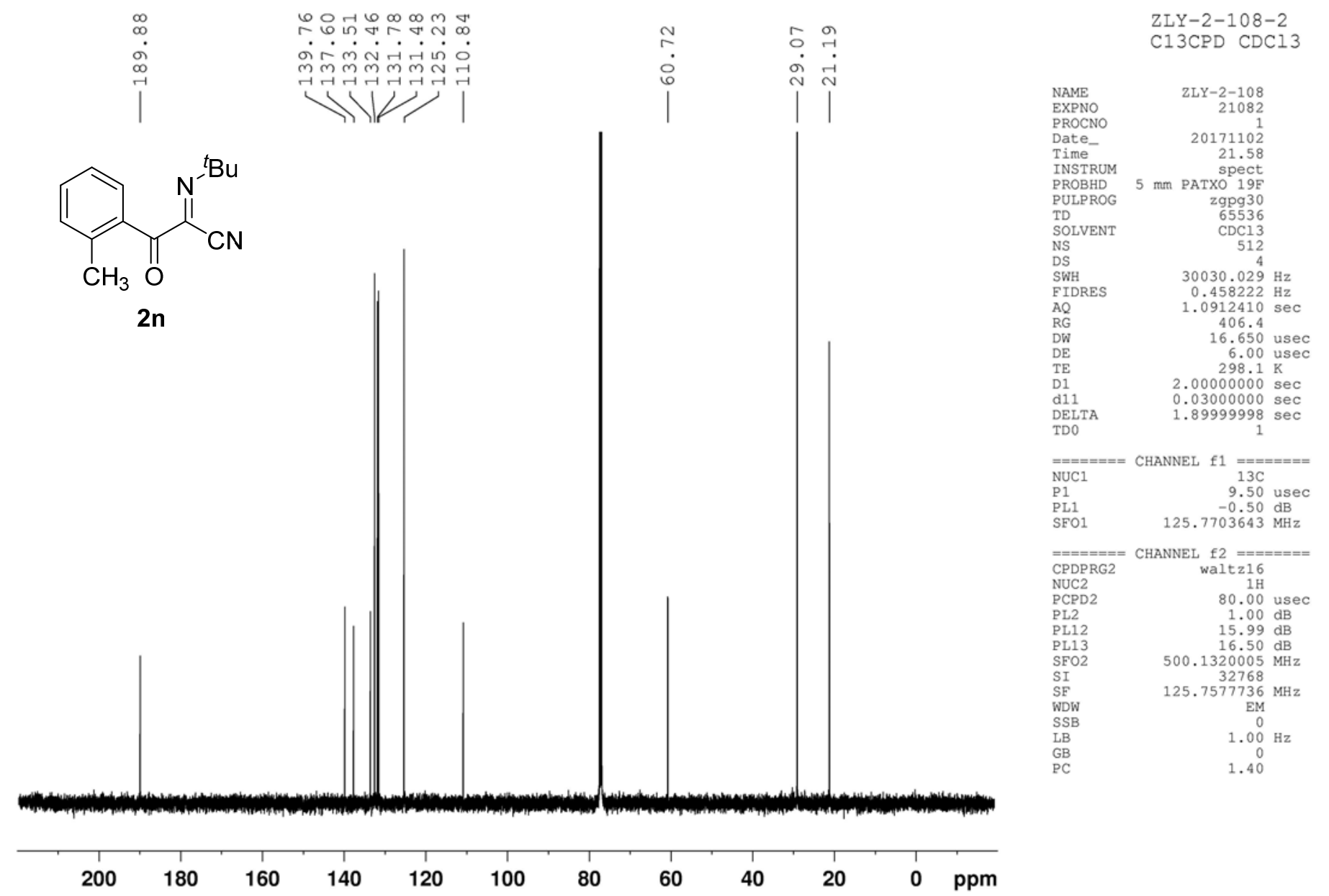




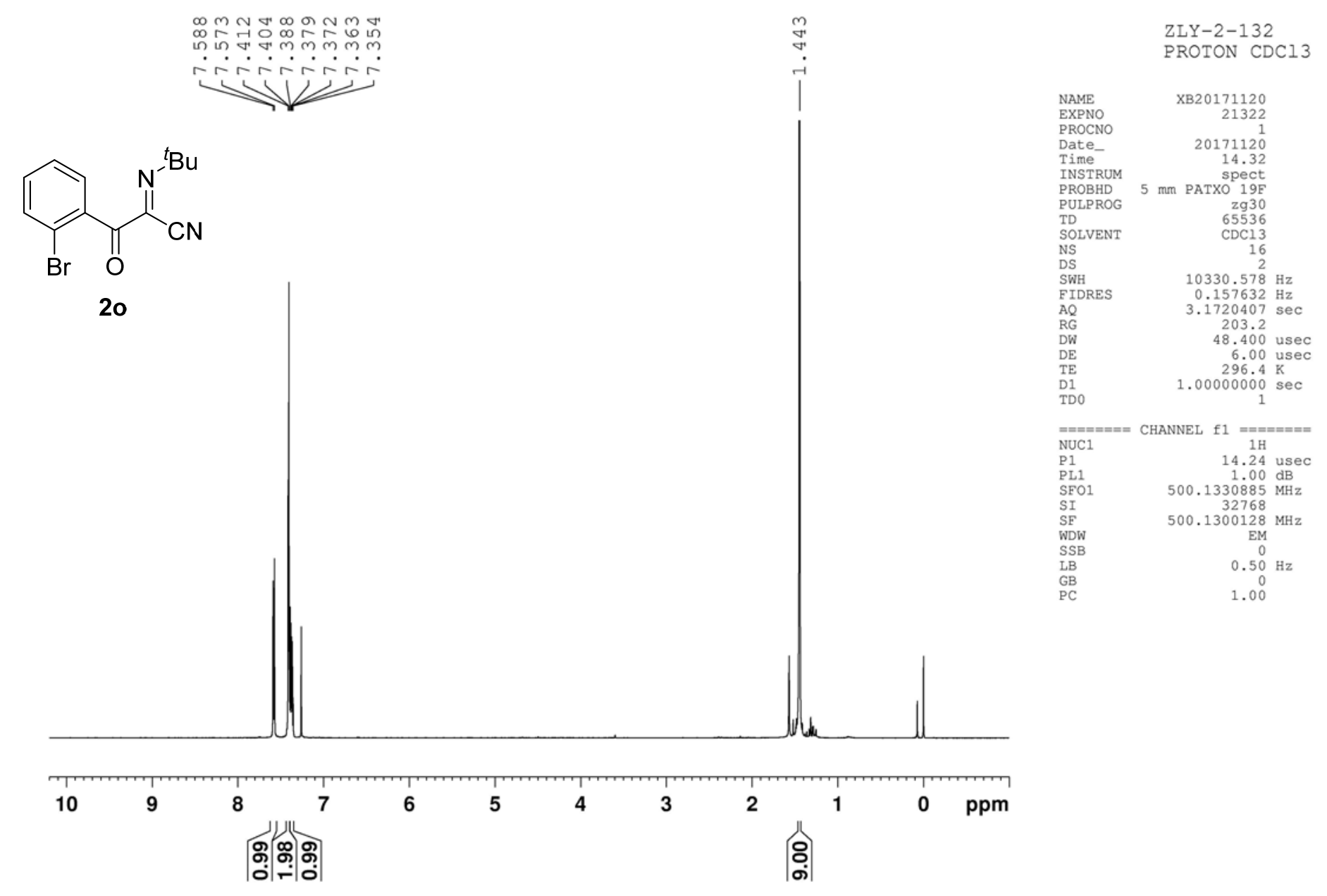




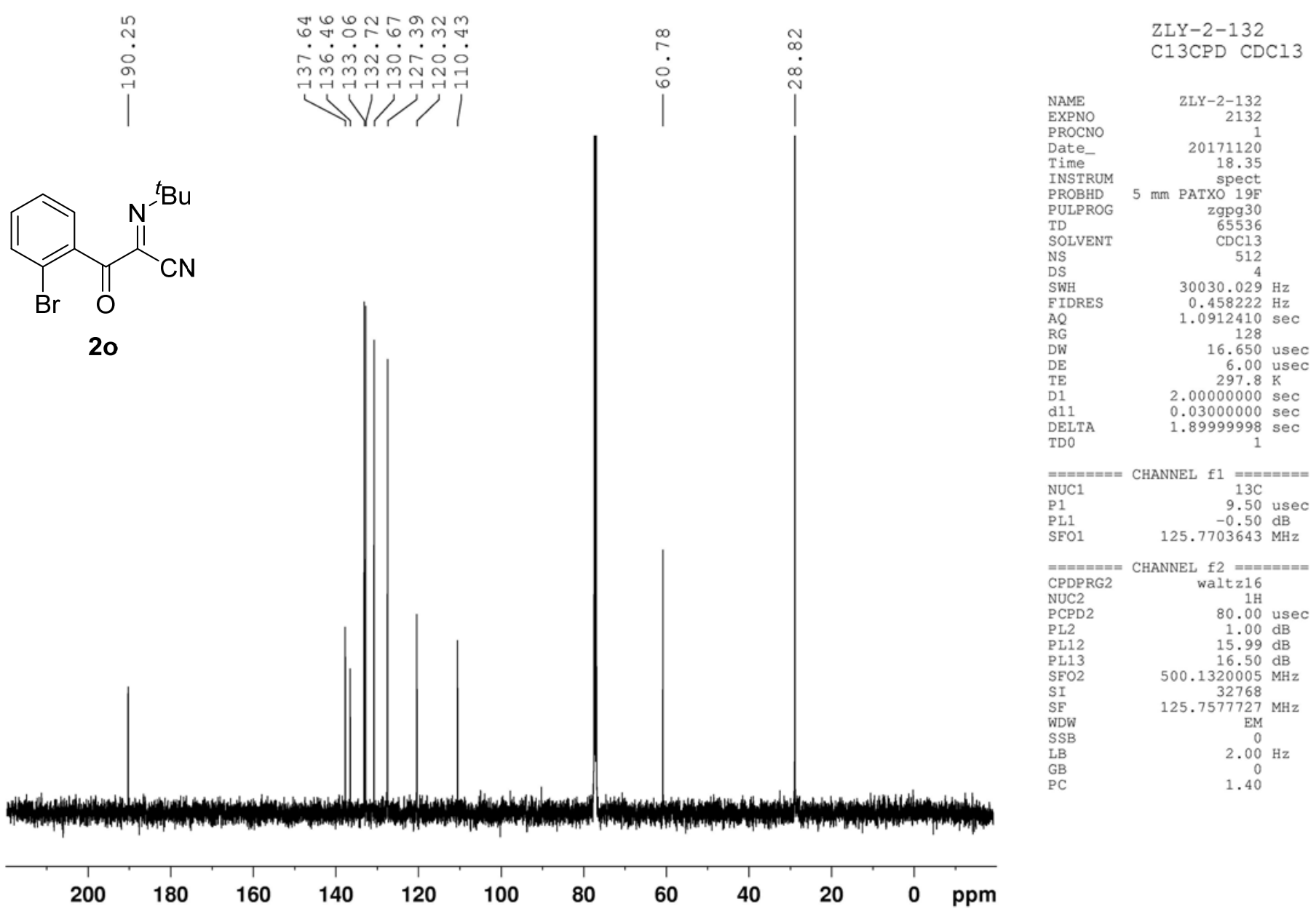




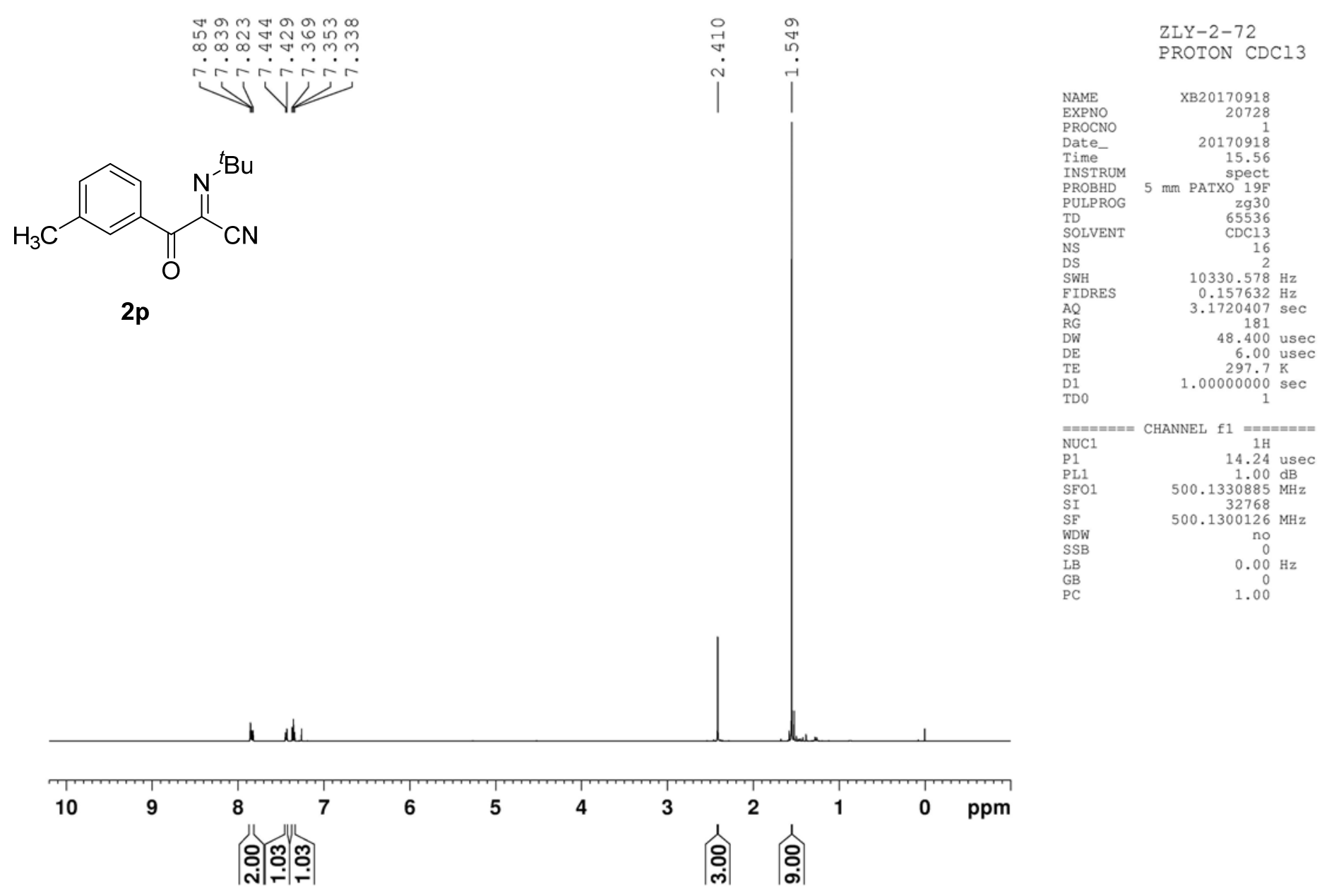




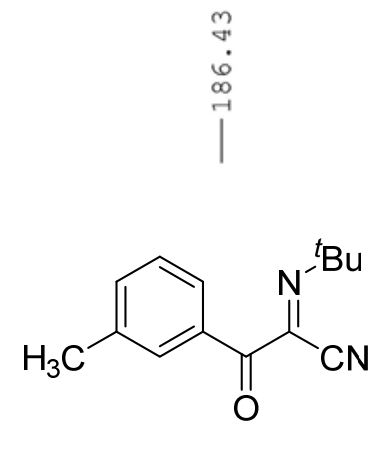

$2 p$

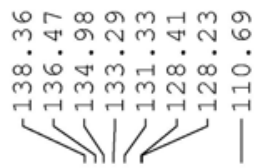

i

ก

$\stackrel{\text { ㄱ }}{\sim}$

|

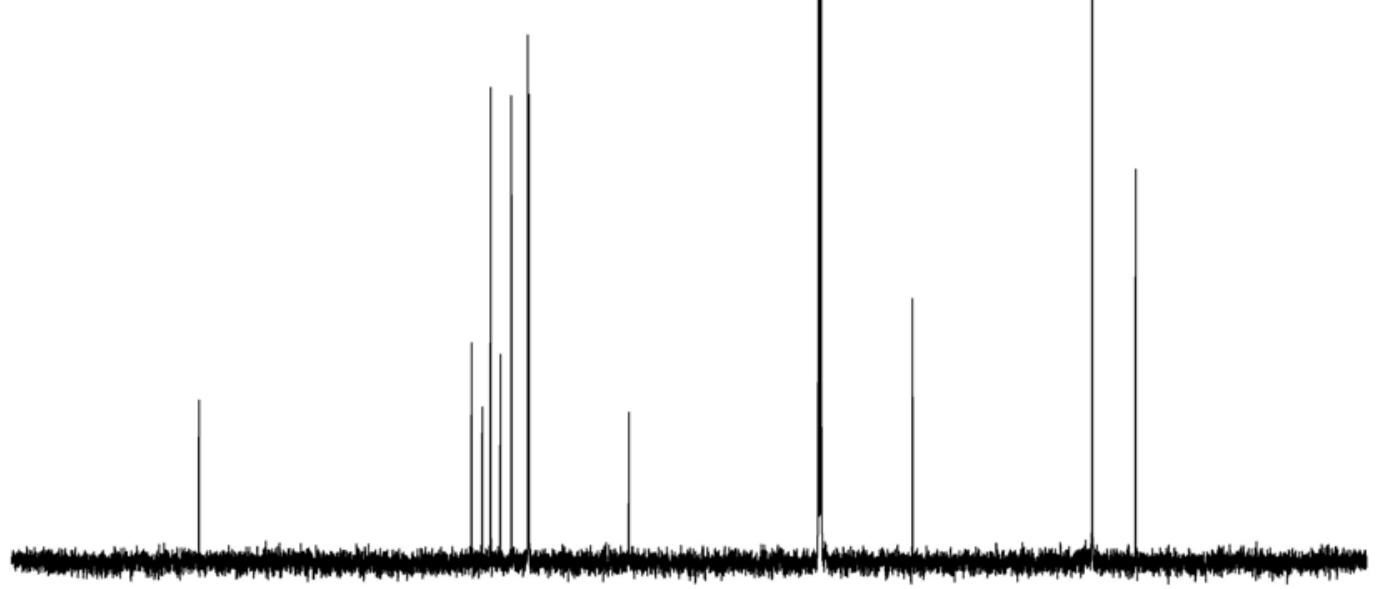

200 

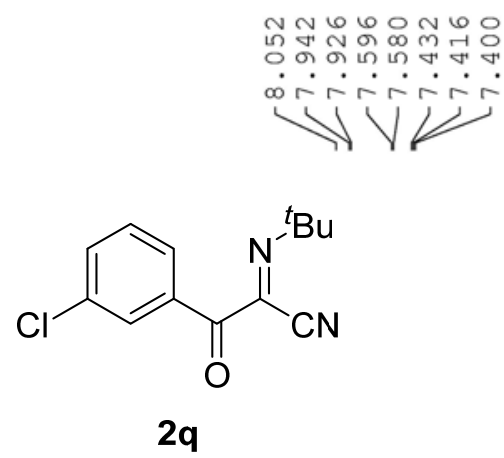

2q

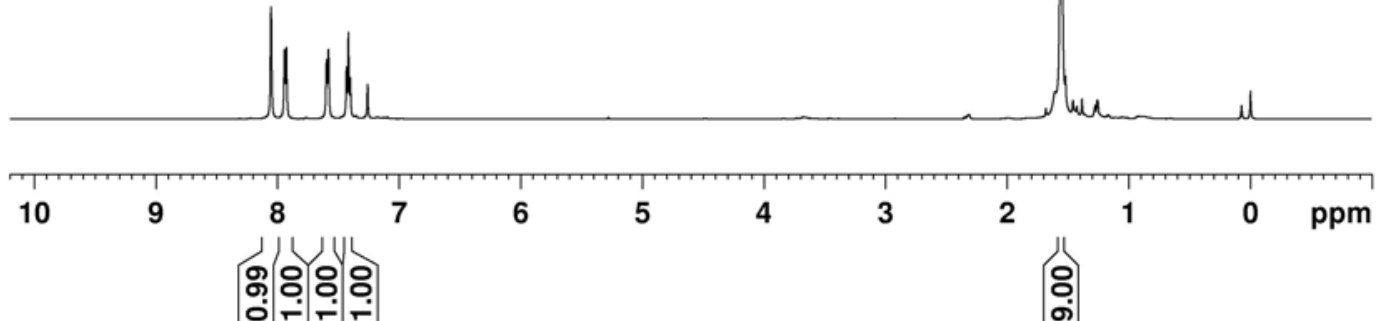

102

PROTON CDCl3

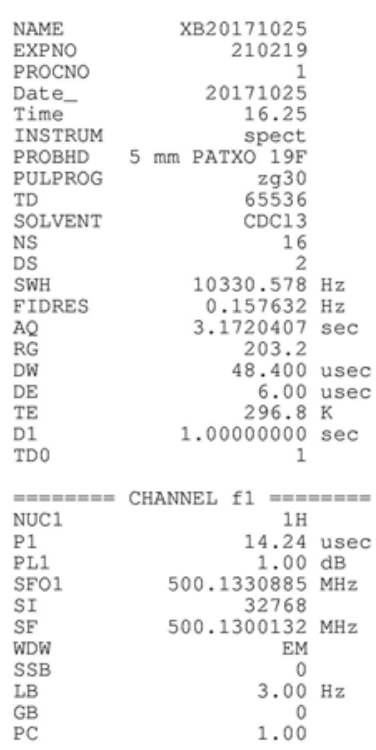

웅훔훔 


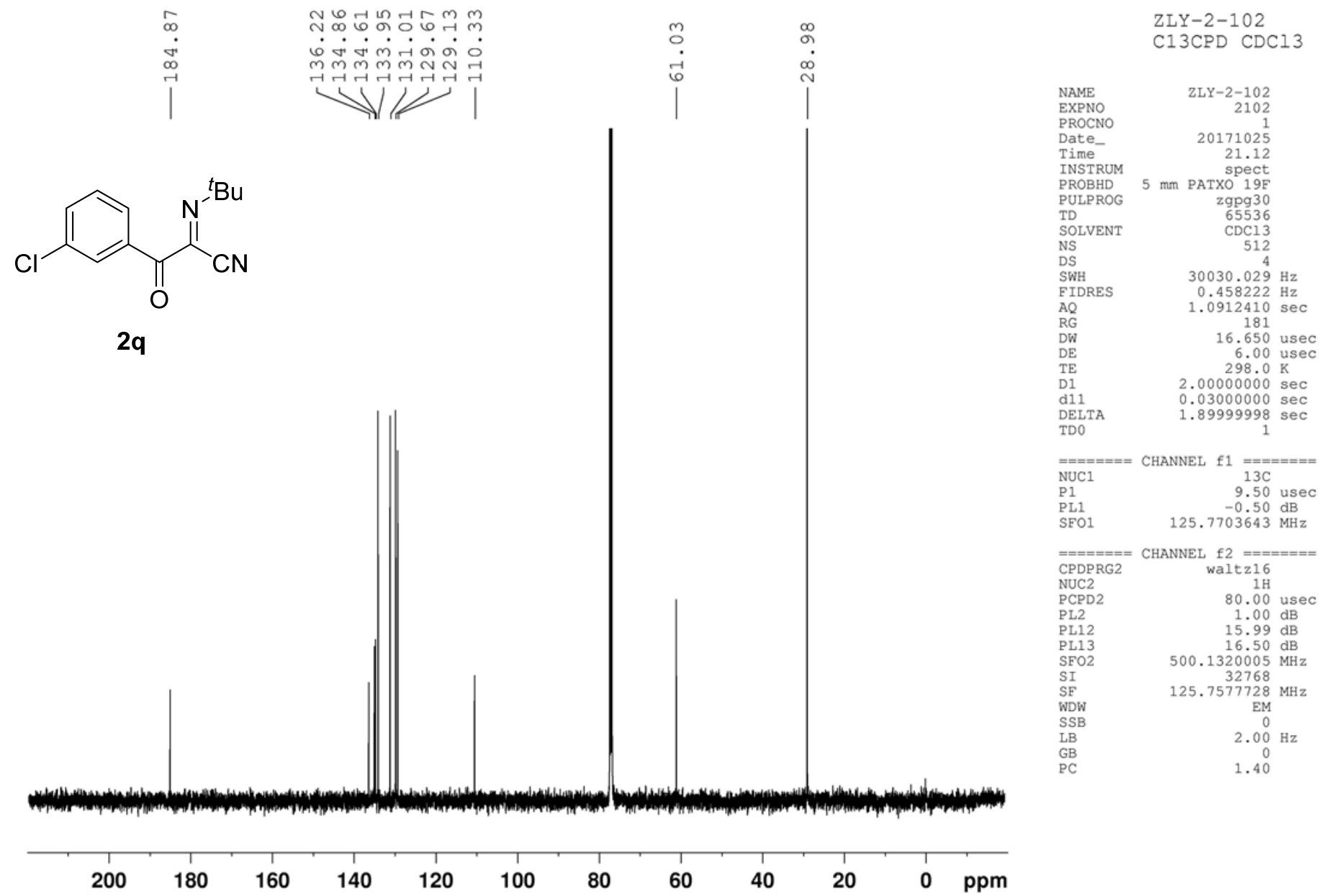




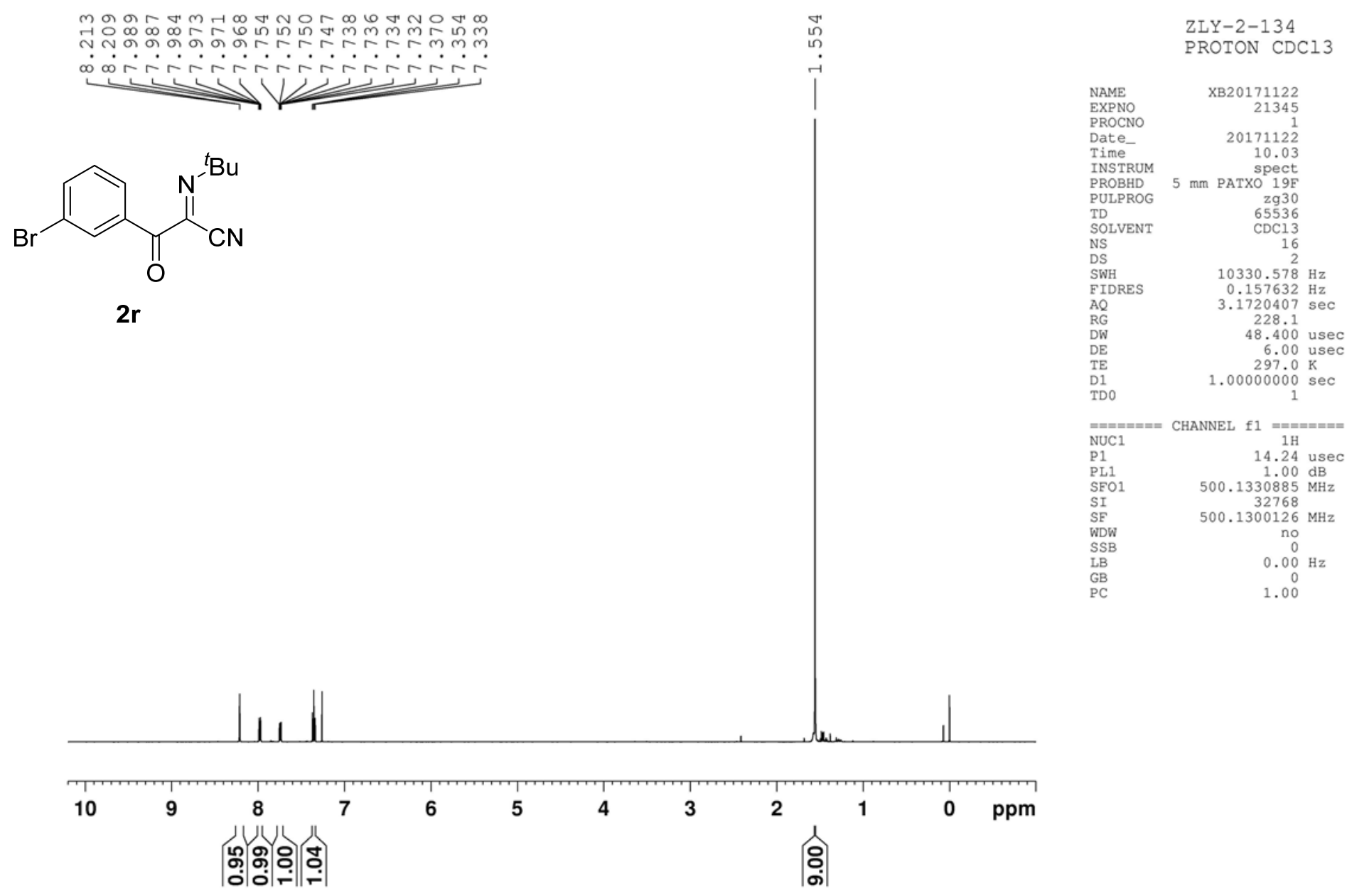




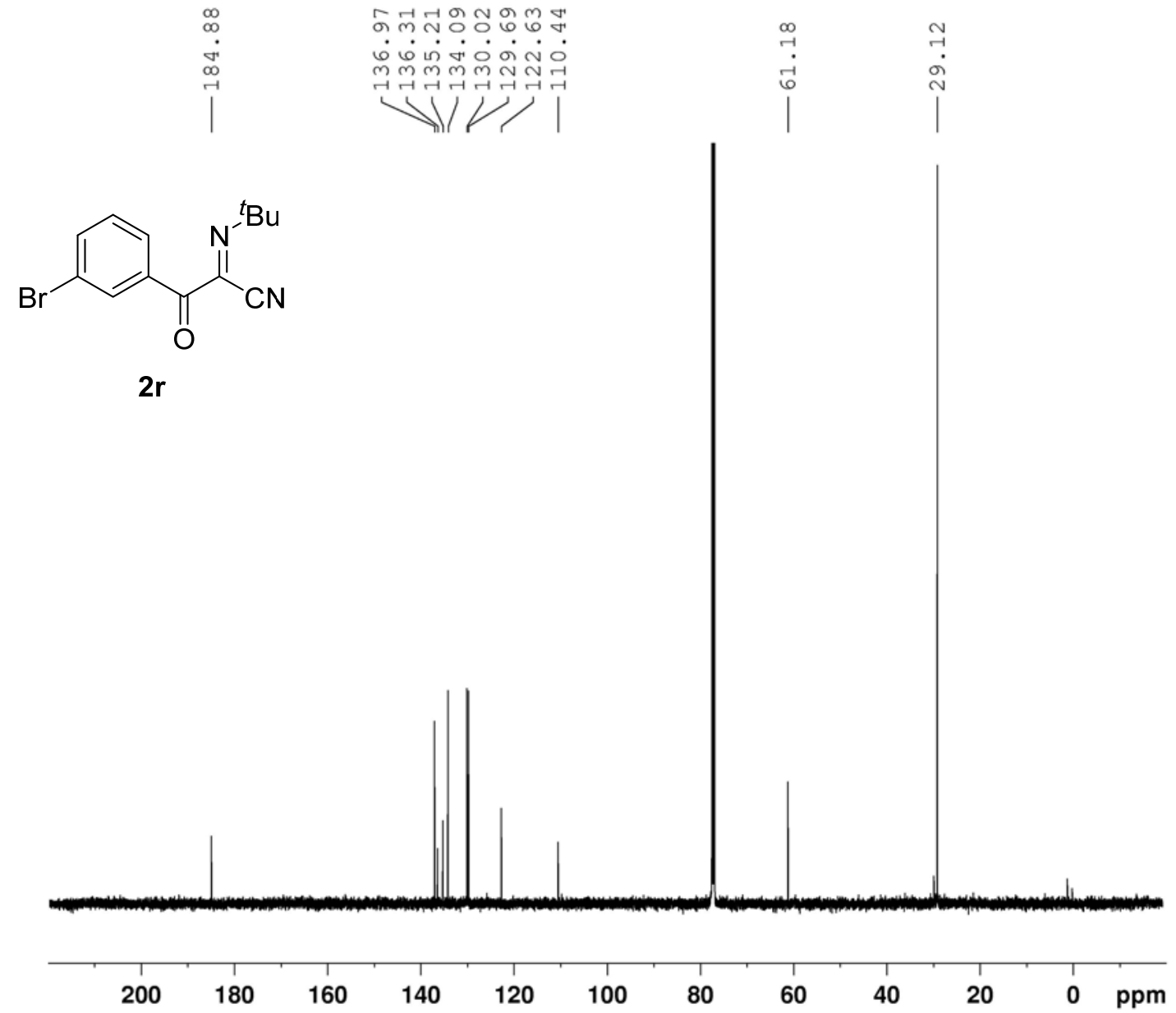

ZLY-2-134

C13CPD CDC13

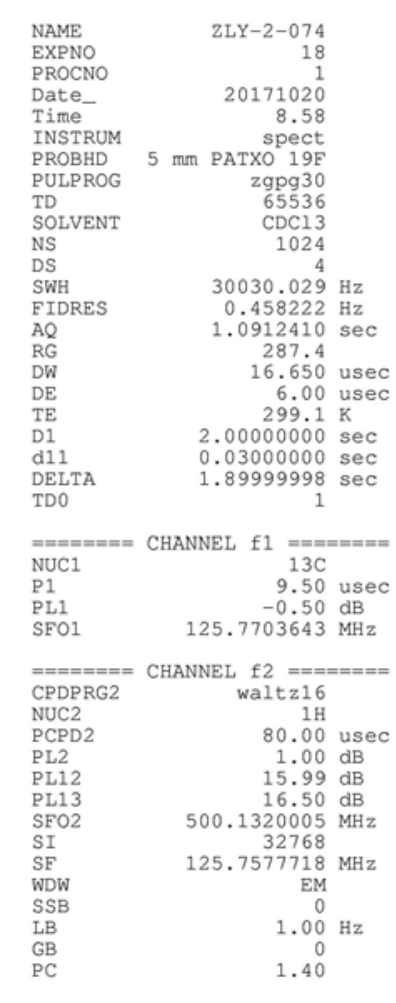




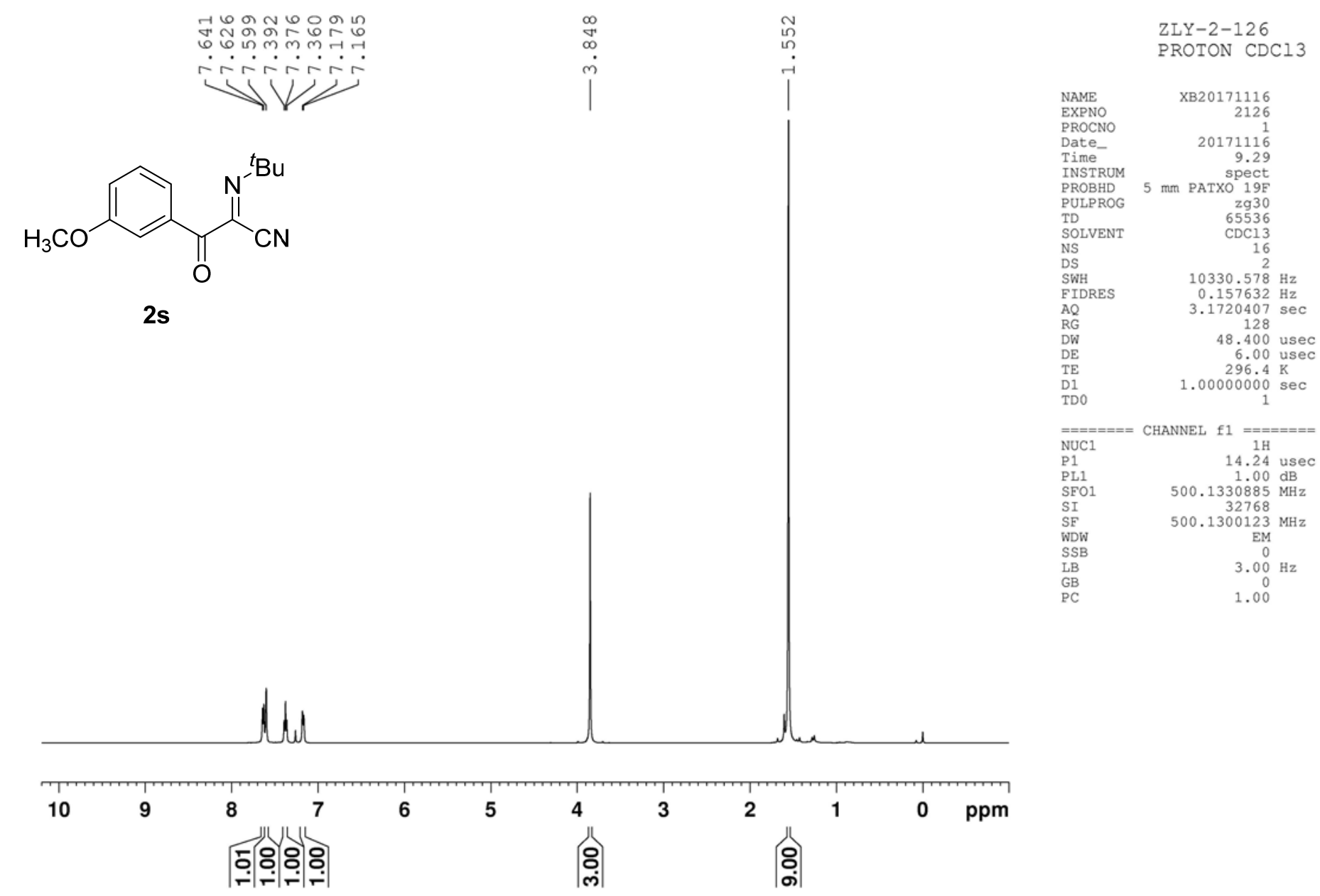




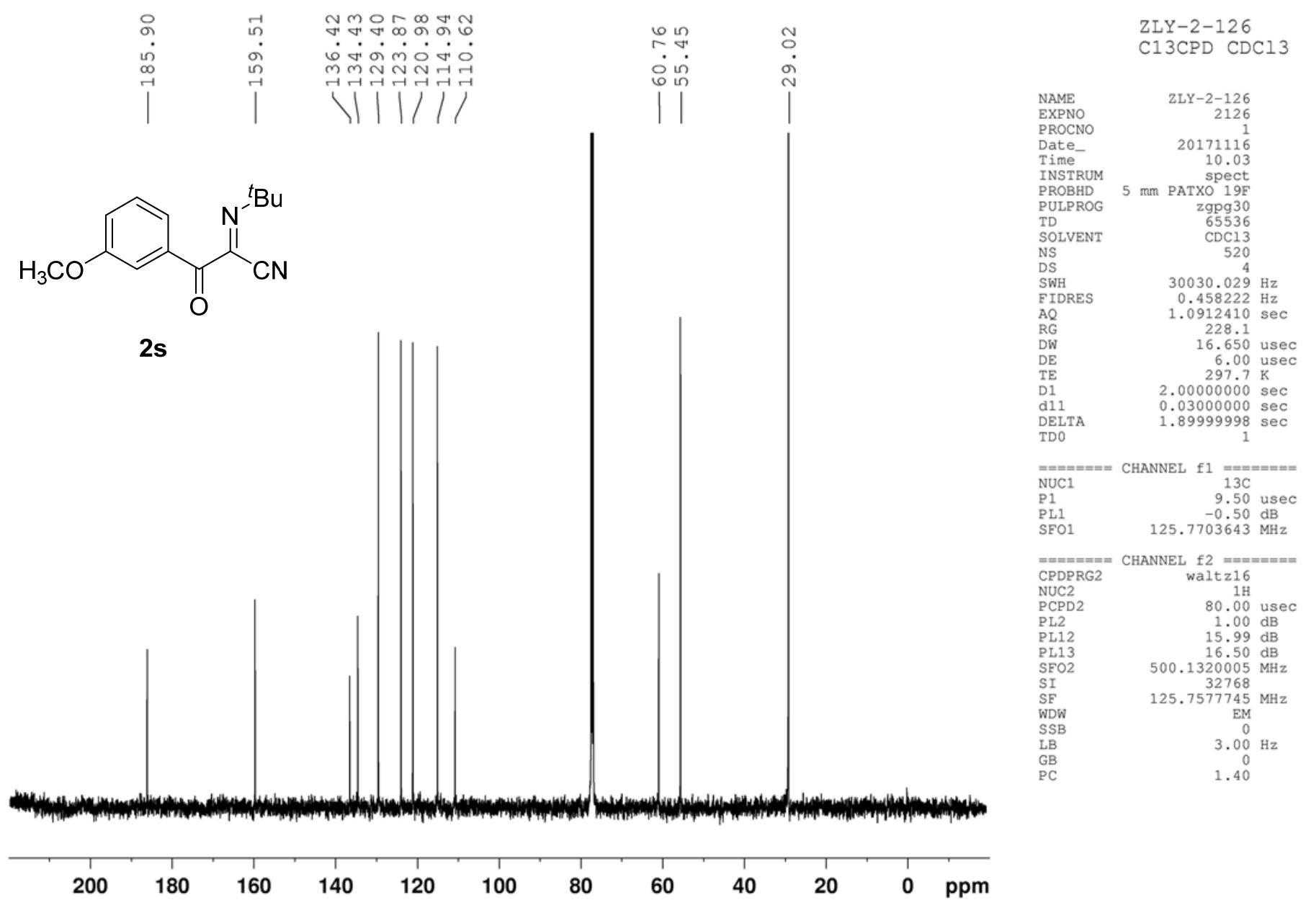




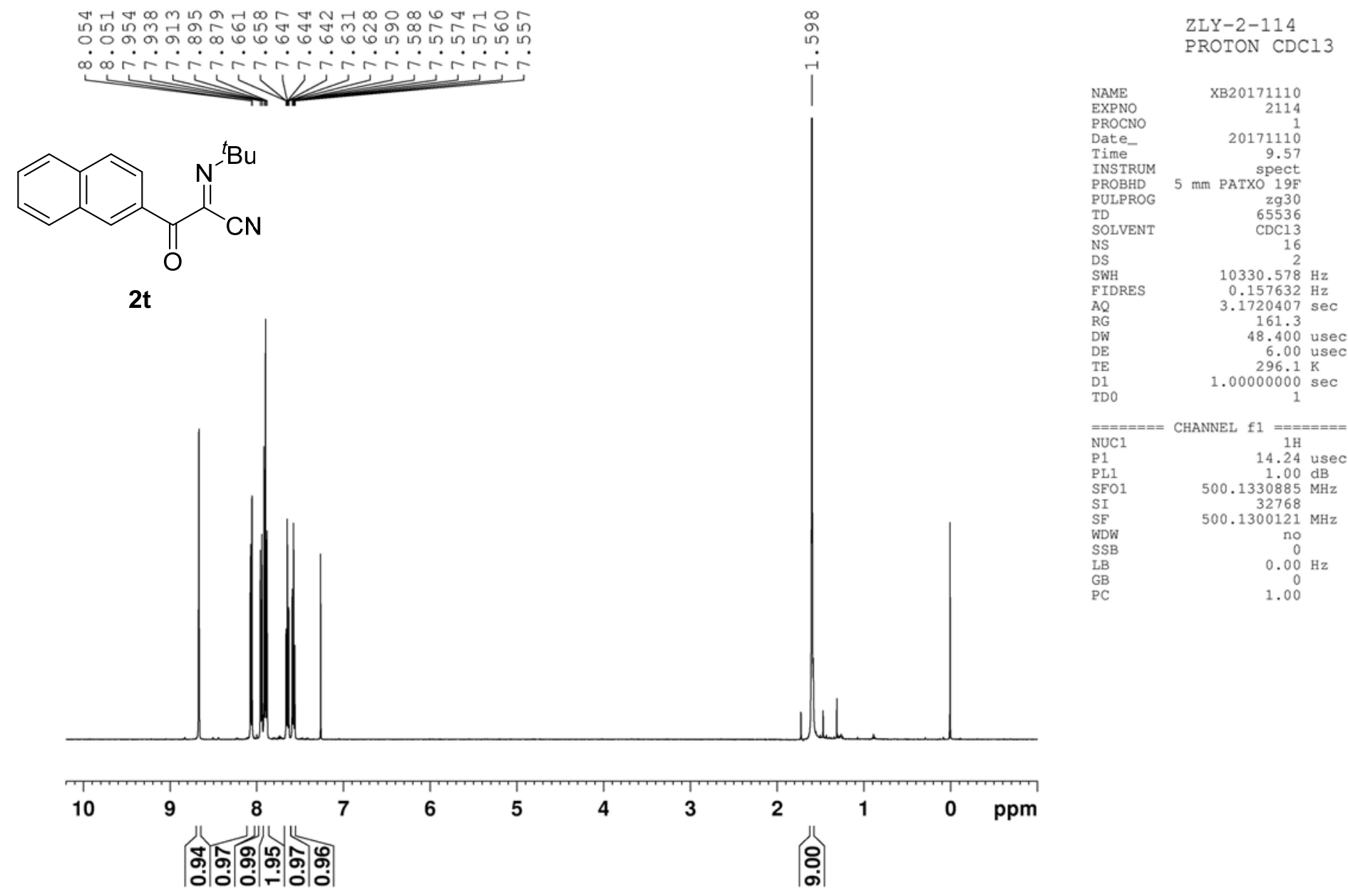




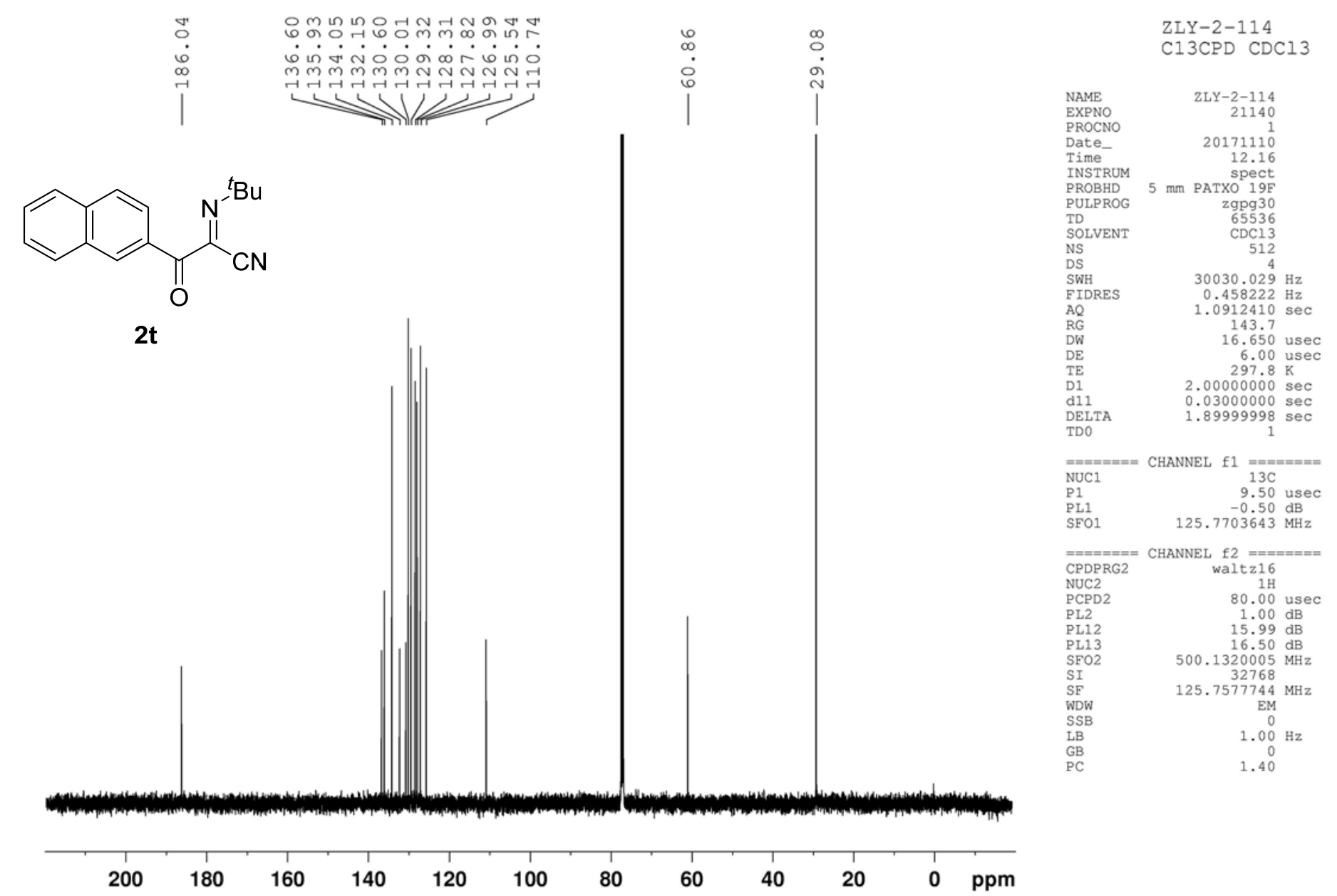




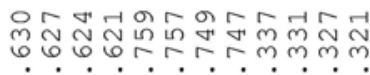

$\underbrace{\infty}$

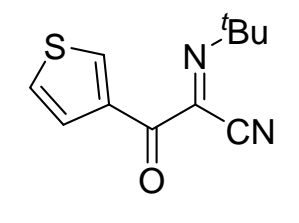

$2 u$

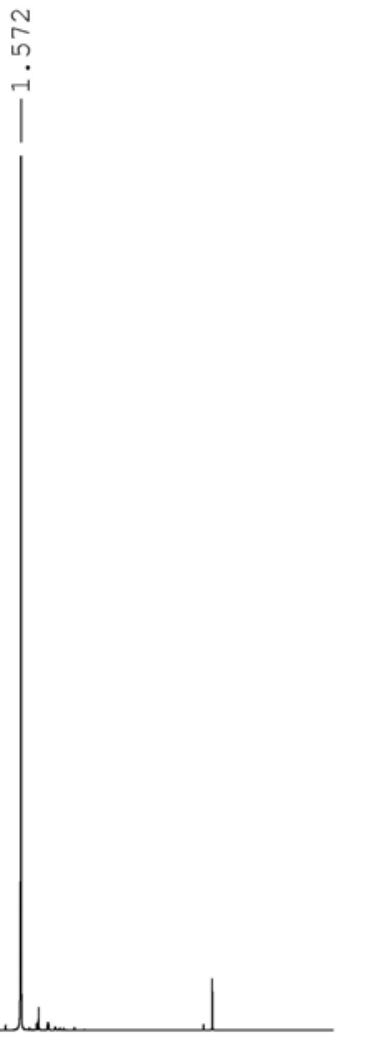

ZLY-3-26
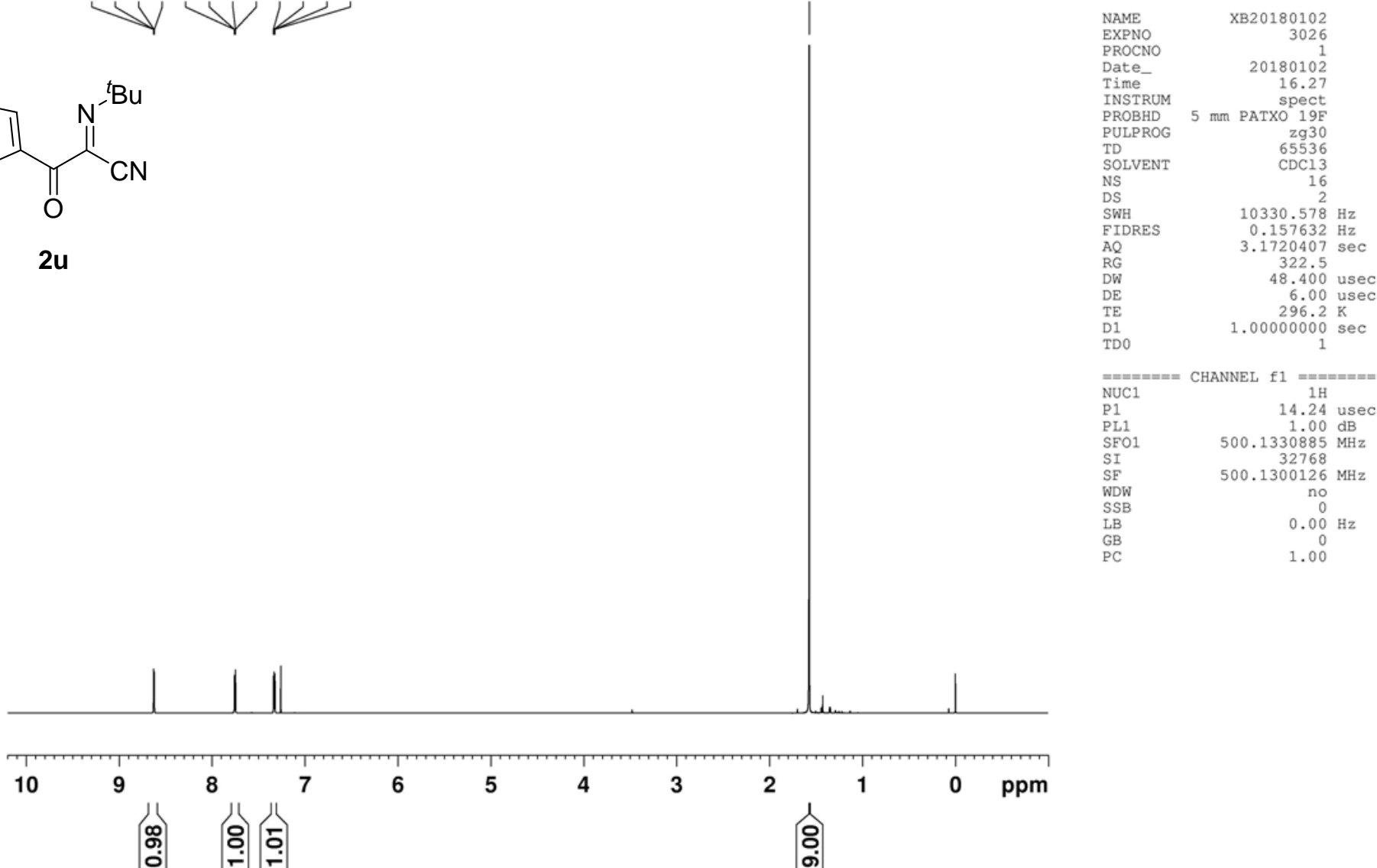


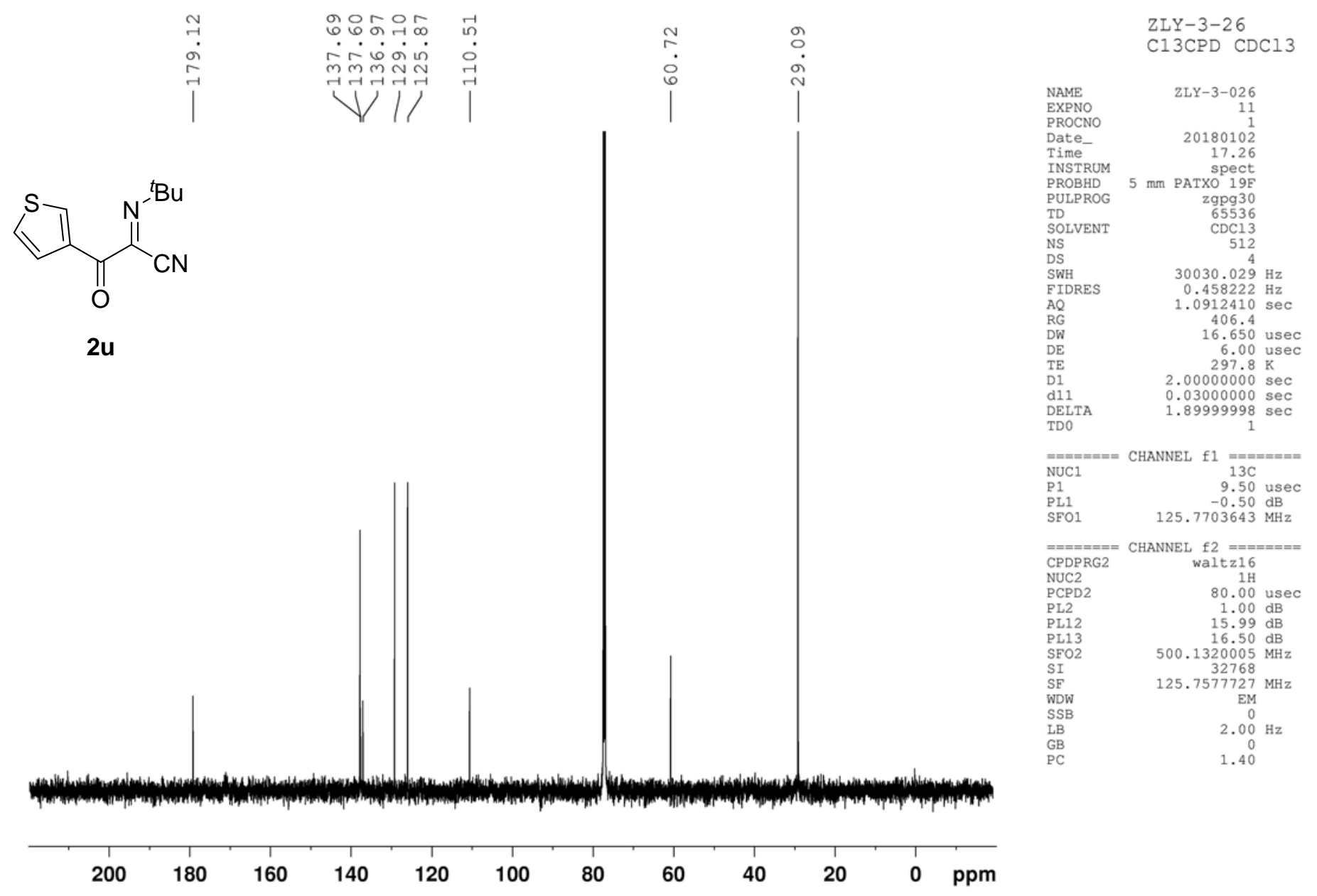




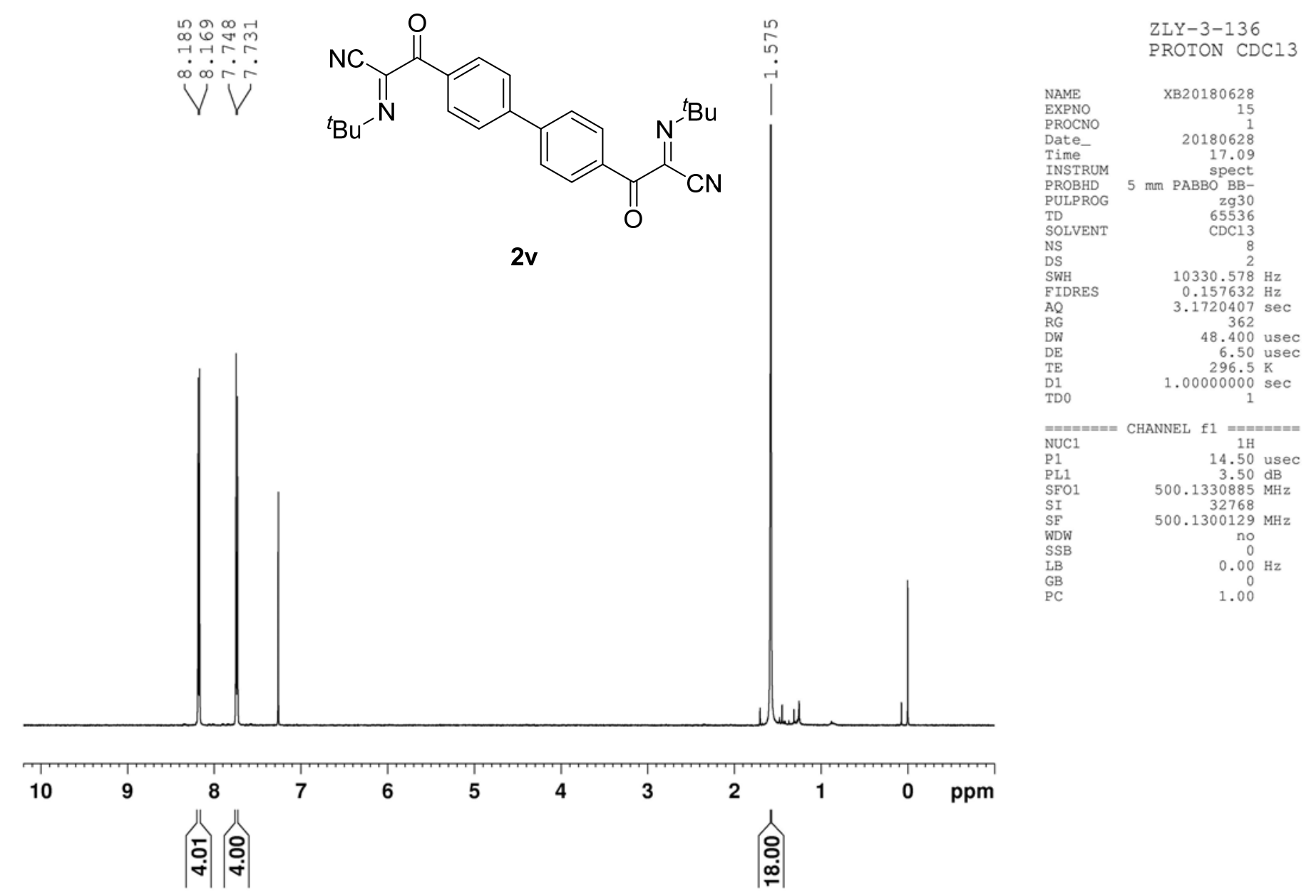




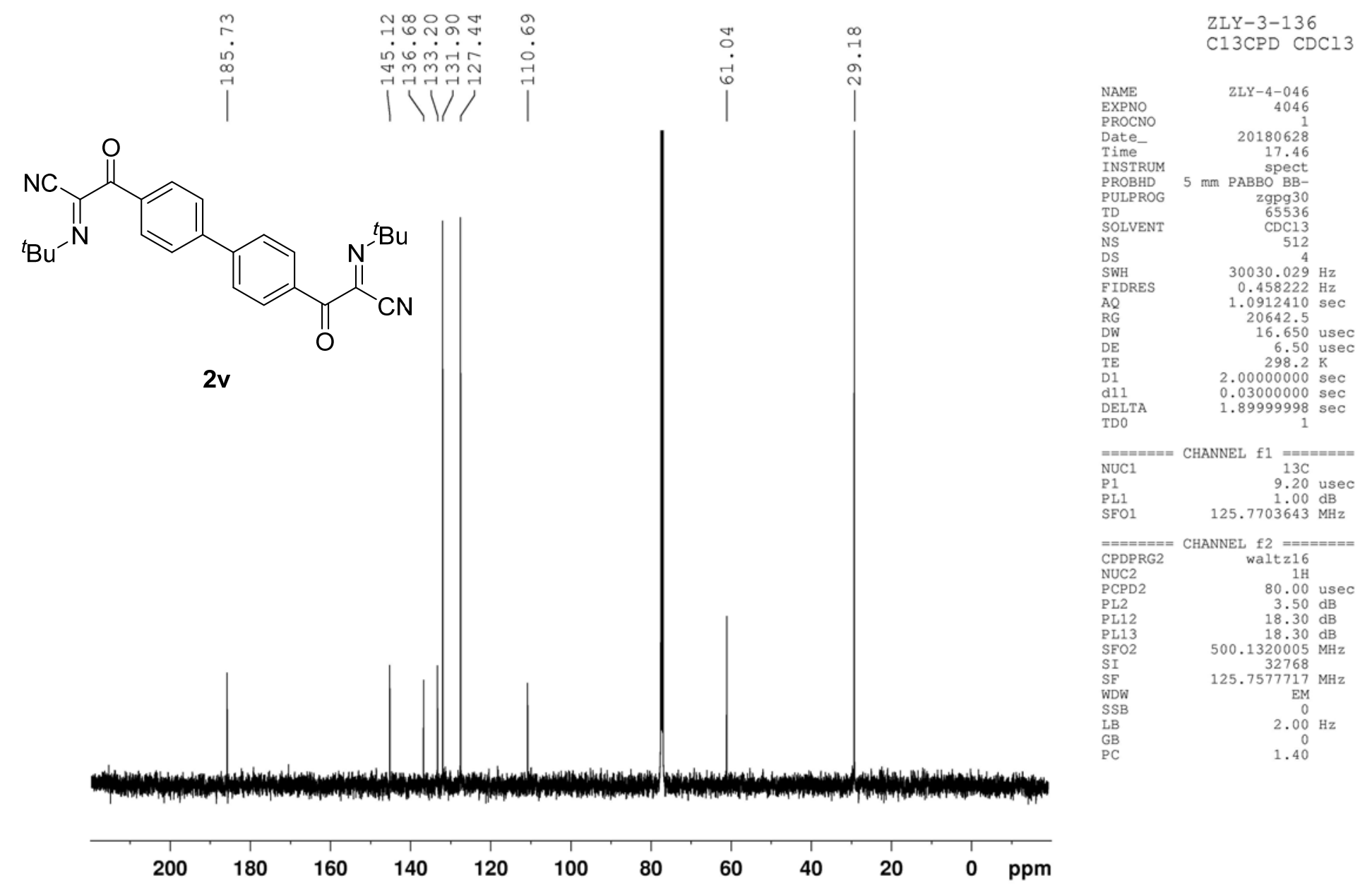




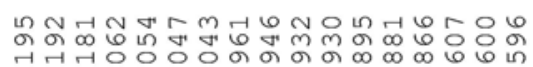

$\infty \infty \infty \infty \infty \infty \infty N-1)$

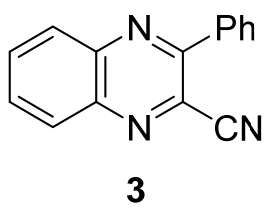

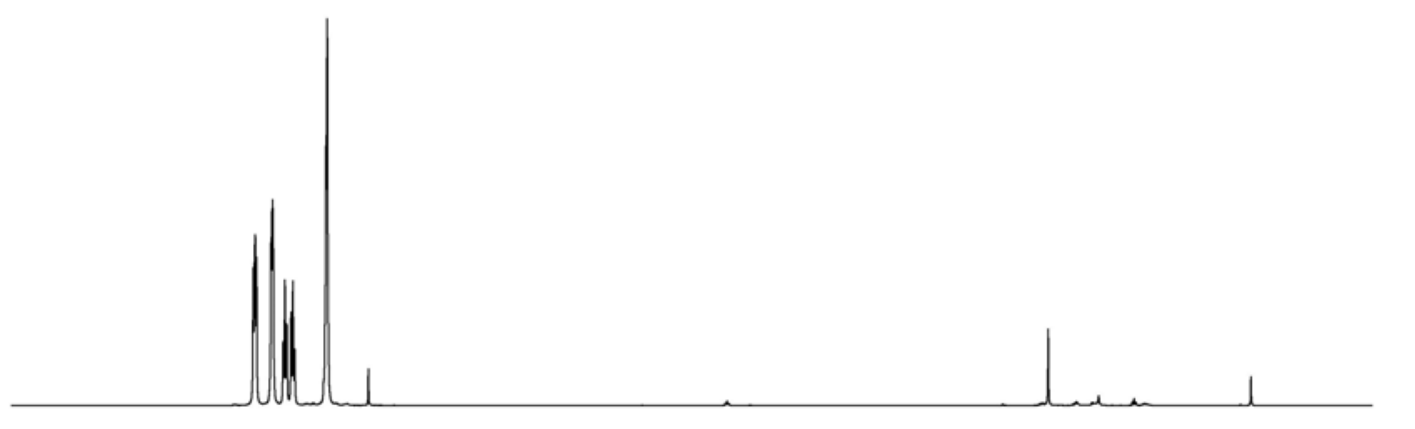

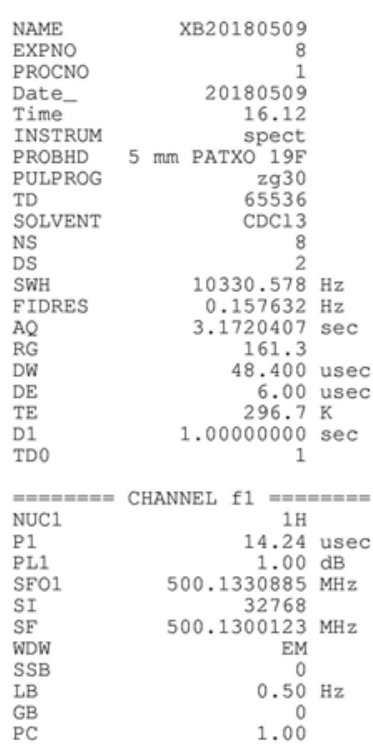

우잉ㅇㅇ응

ZLY-4-4

ROTON CDCl 3

ก่ن 

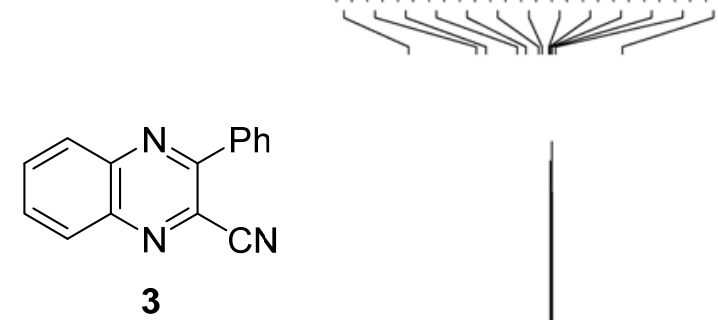

(1)

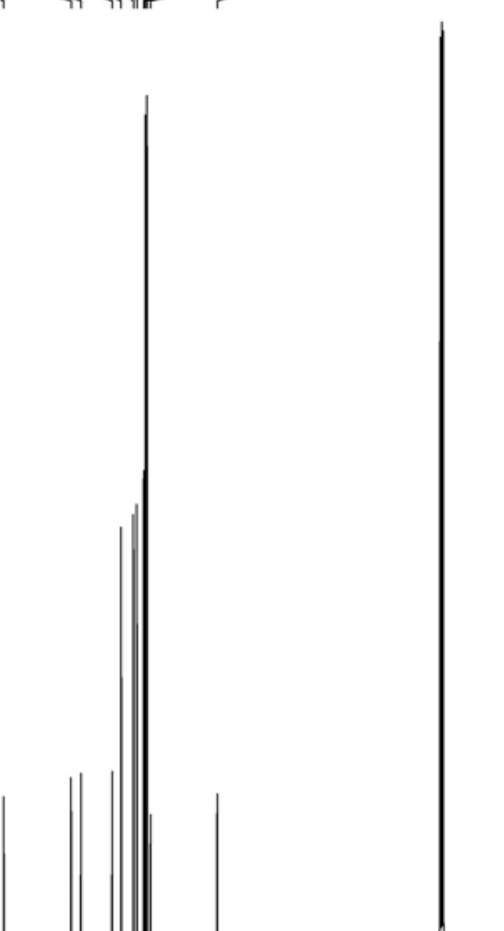

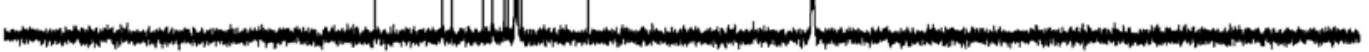

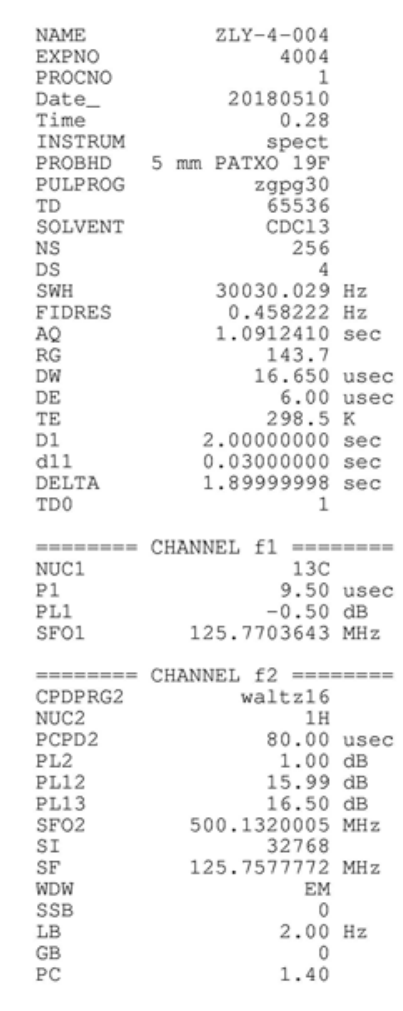




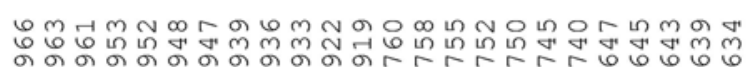

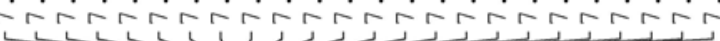
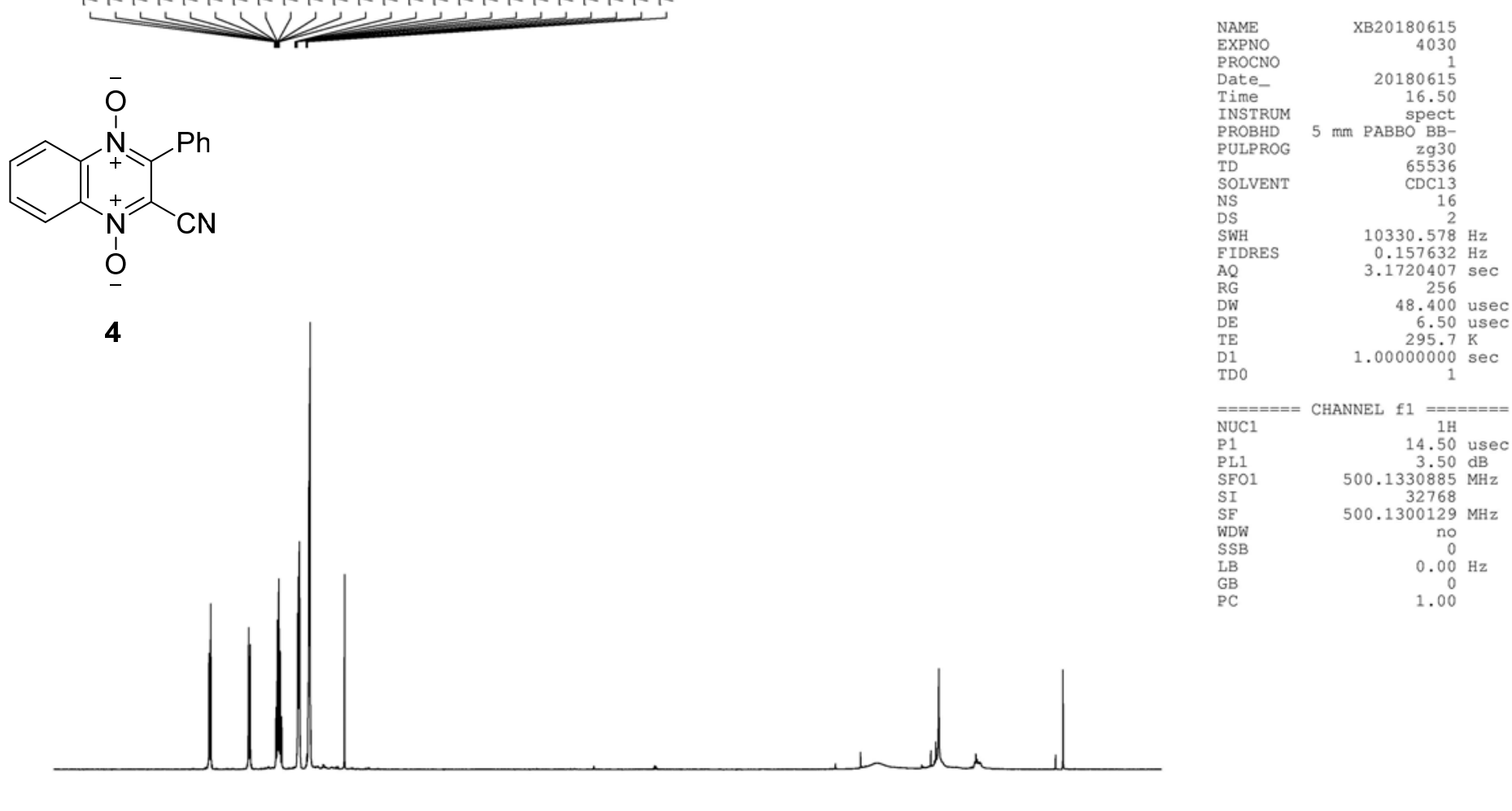

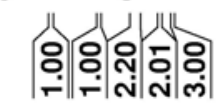




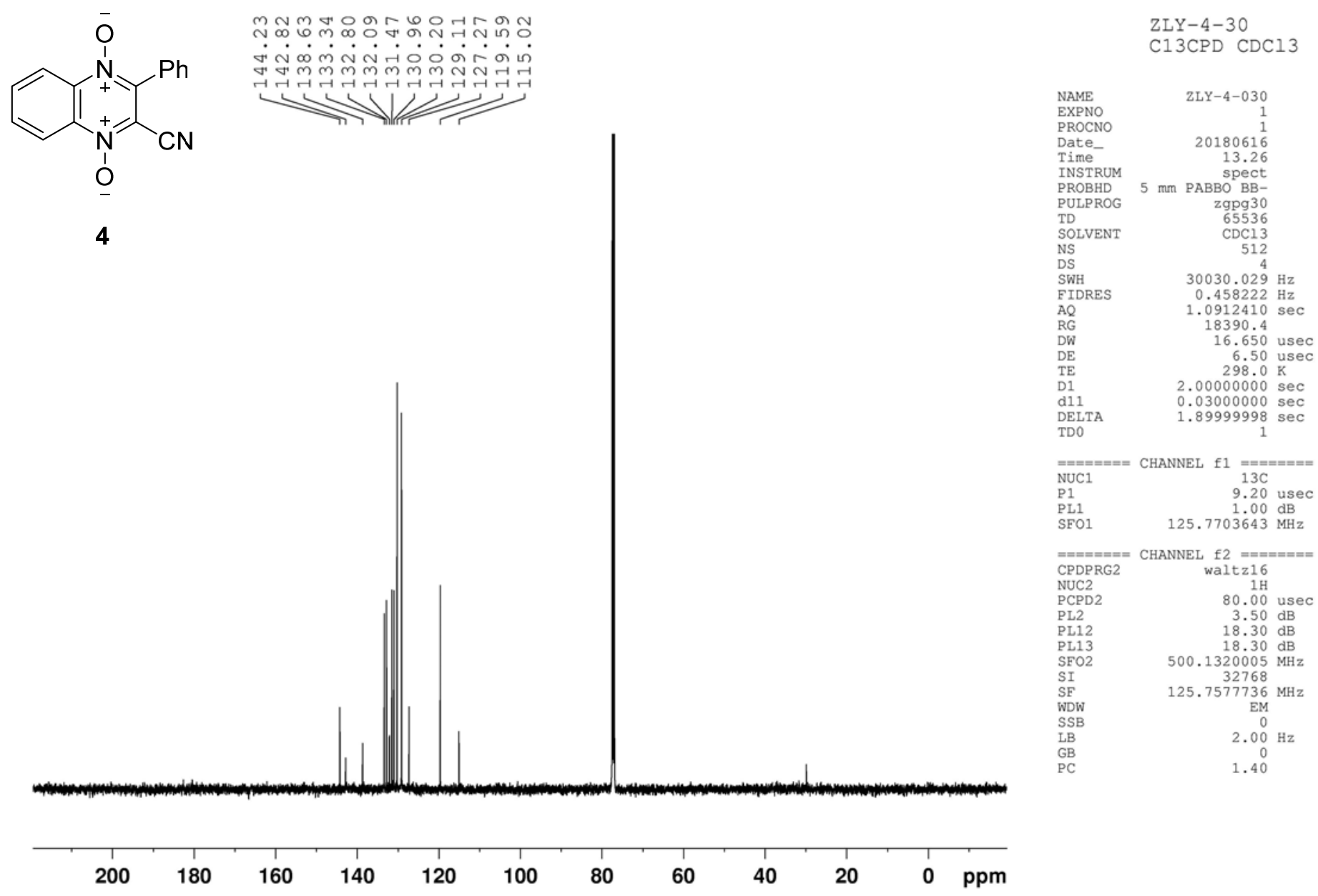




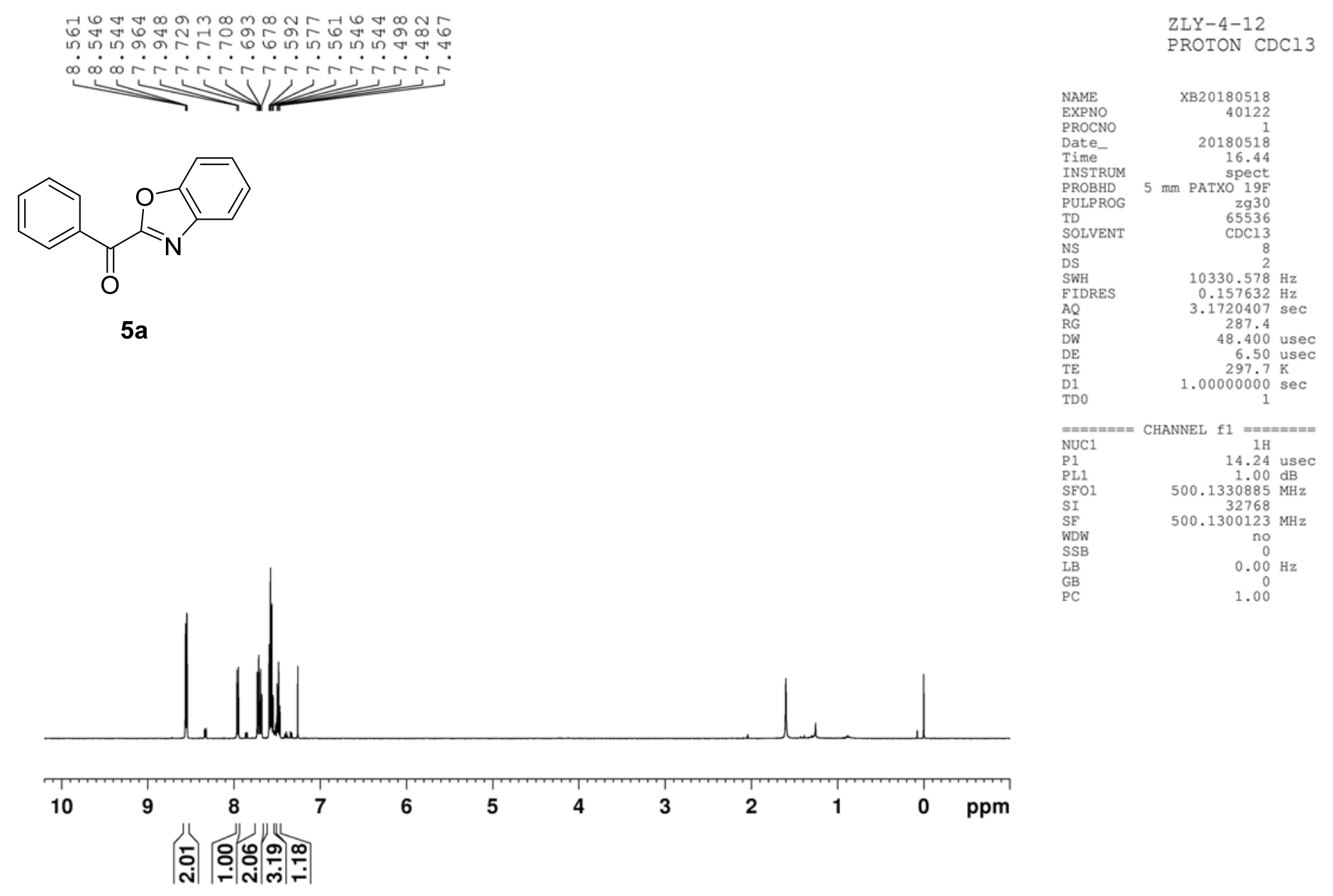



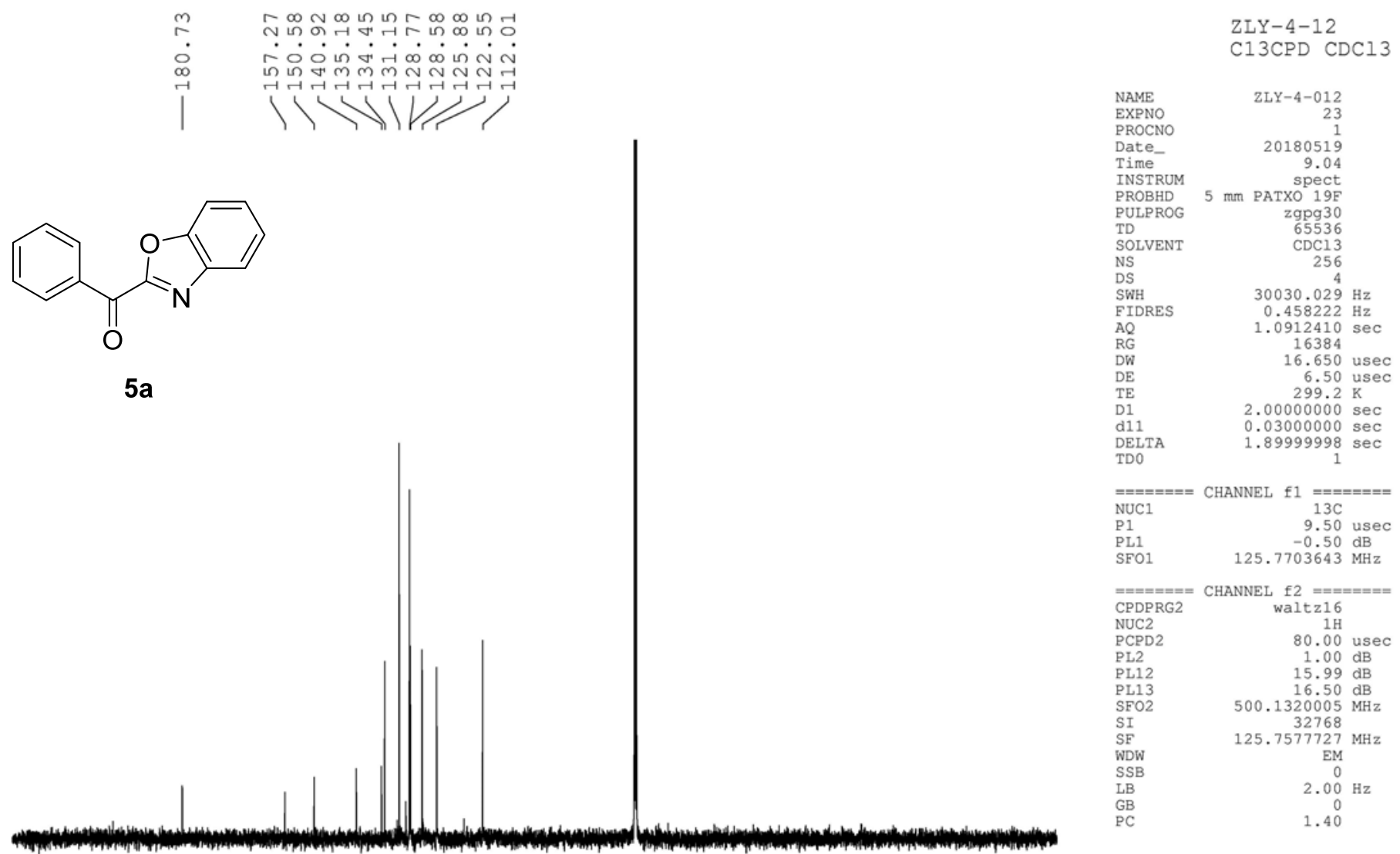

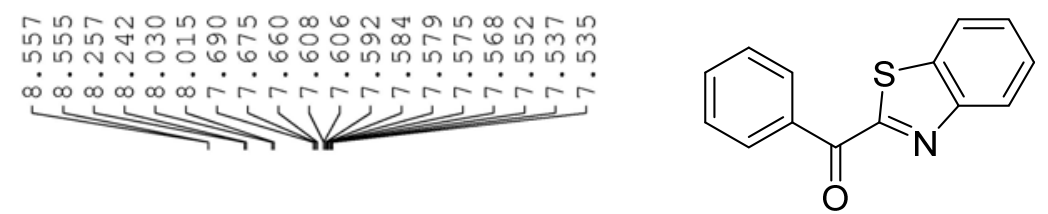

ZLY-5-128

$5 b$

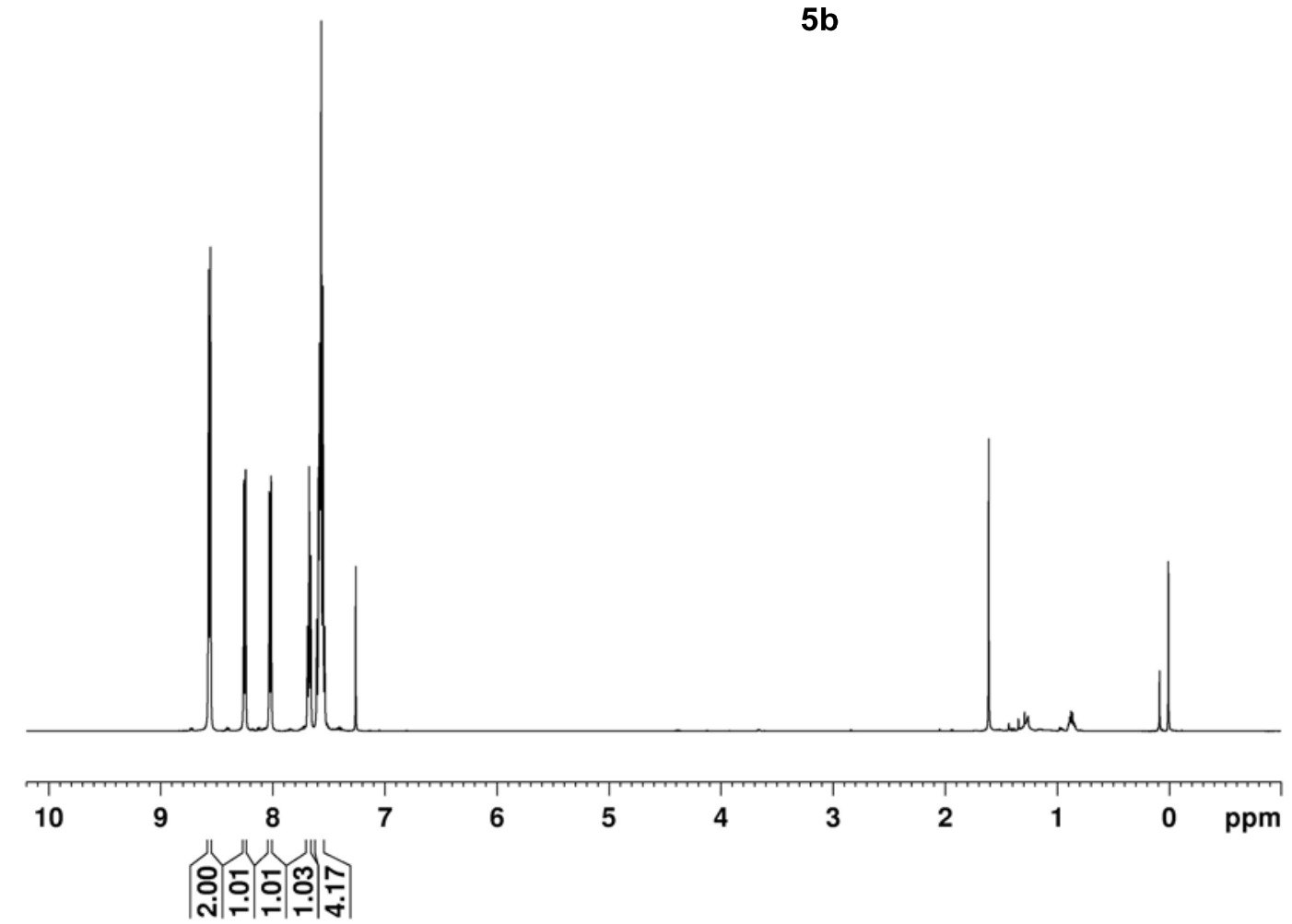




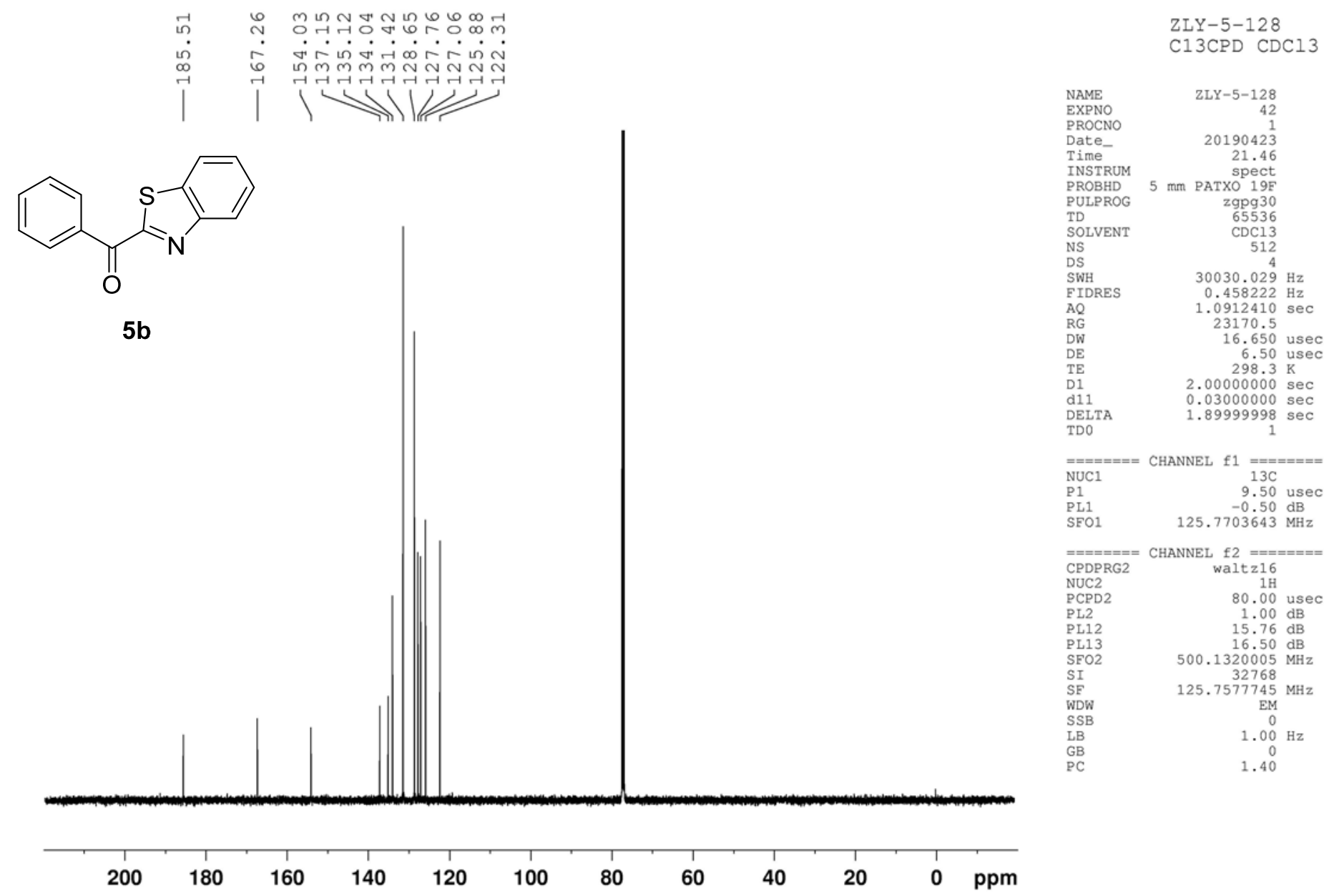




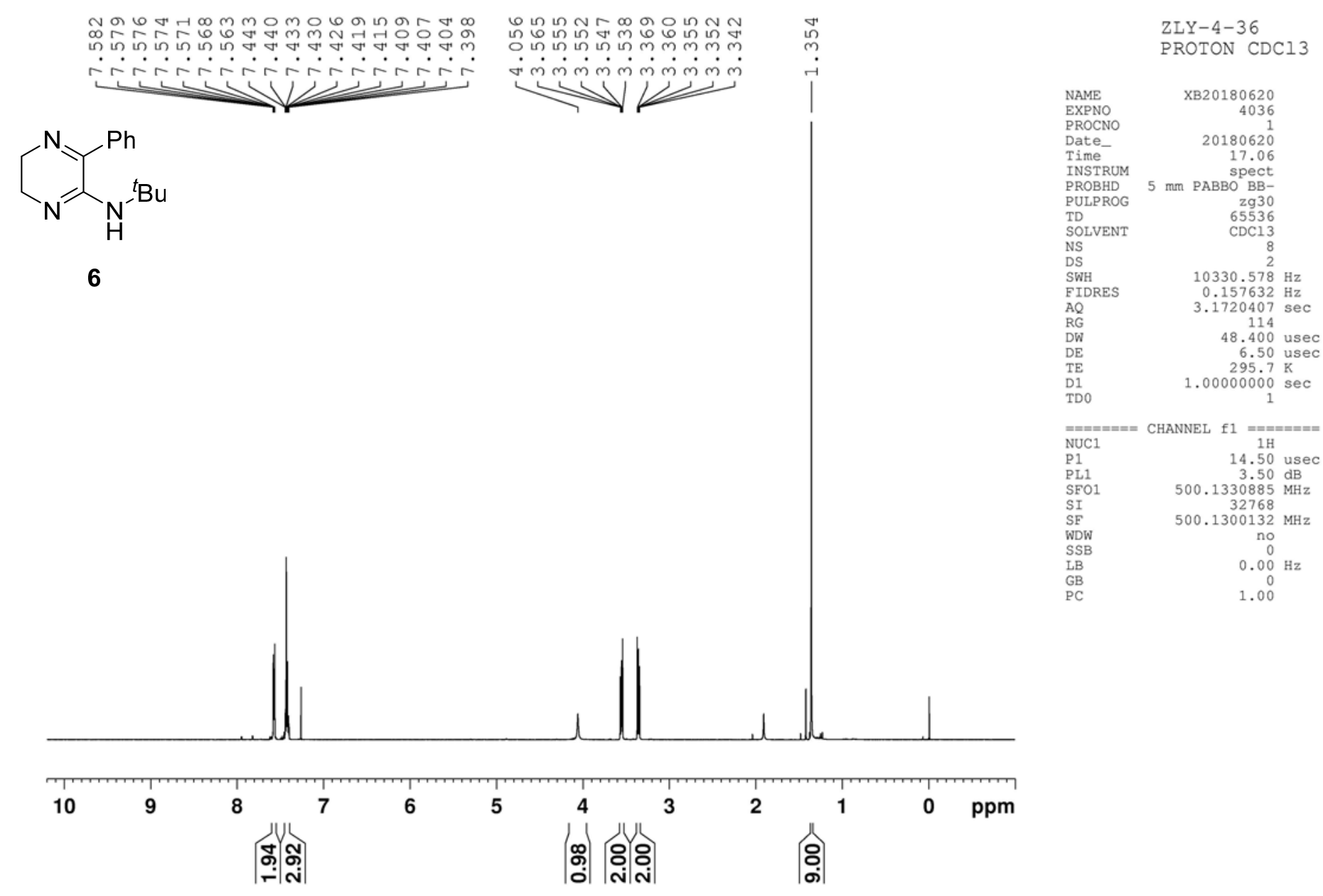




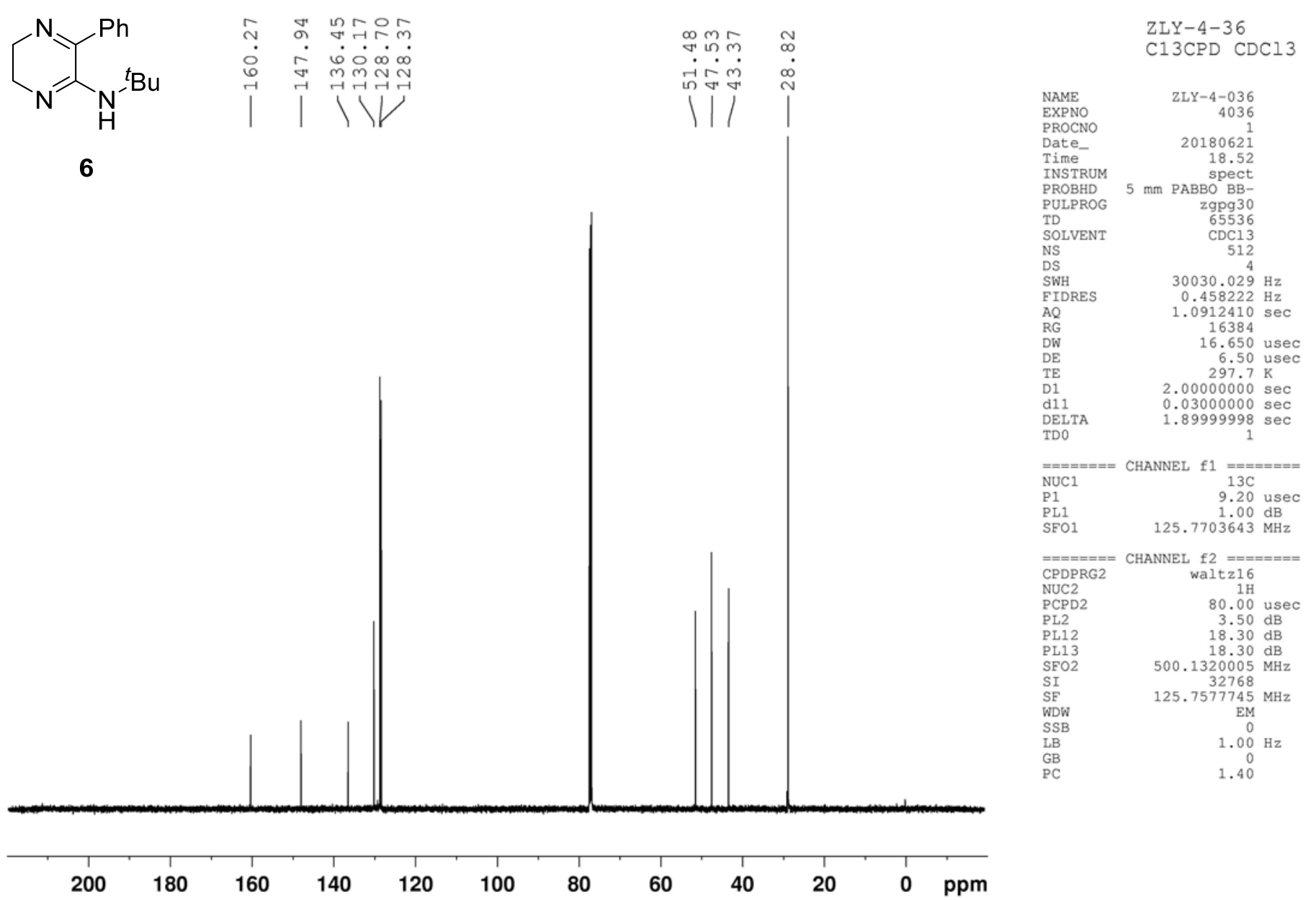




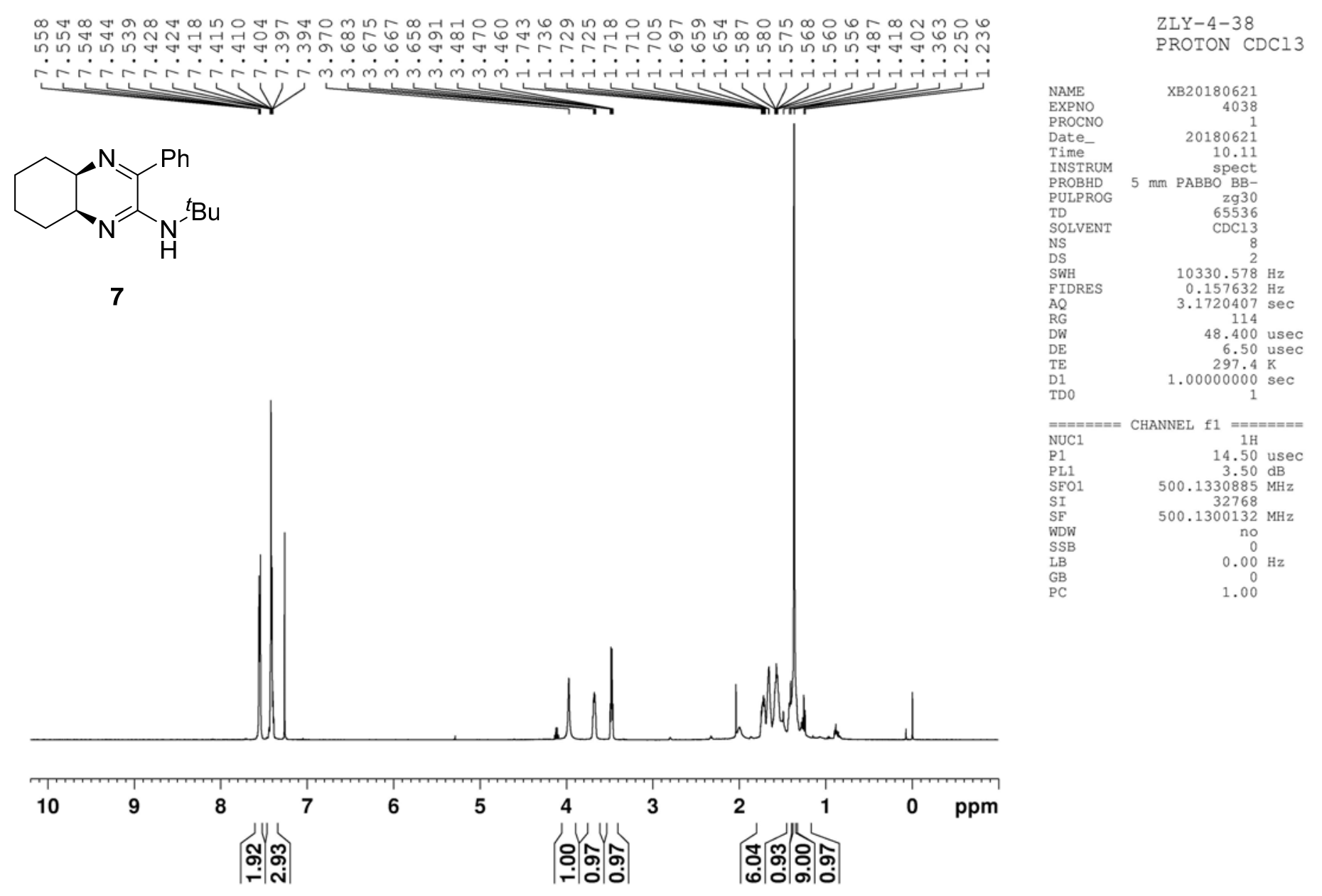




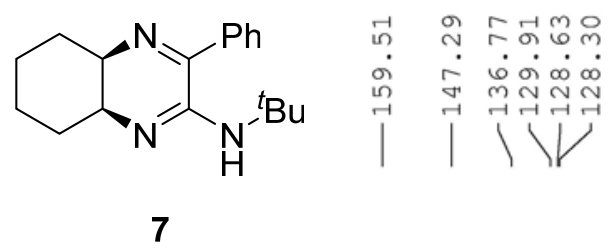

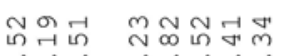

ถู่

ZLY-4-38

C13CPD CDC13

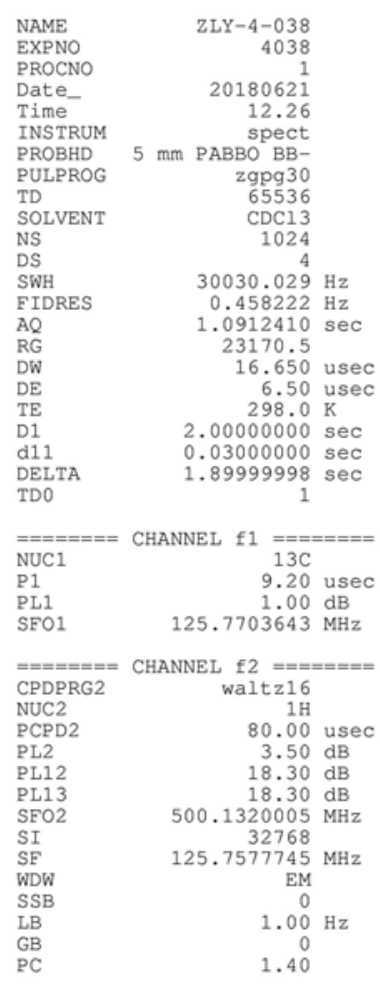




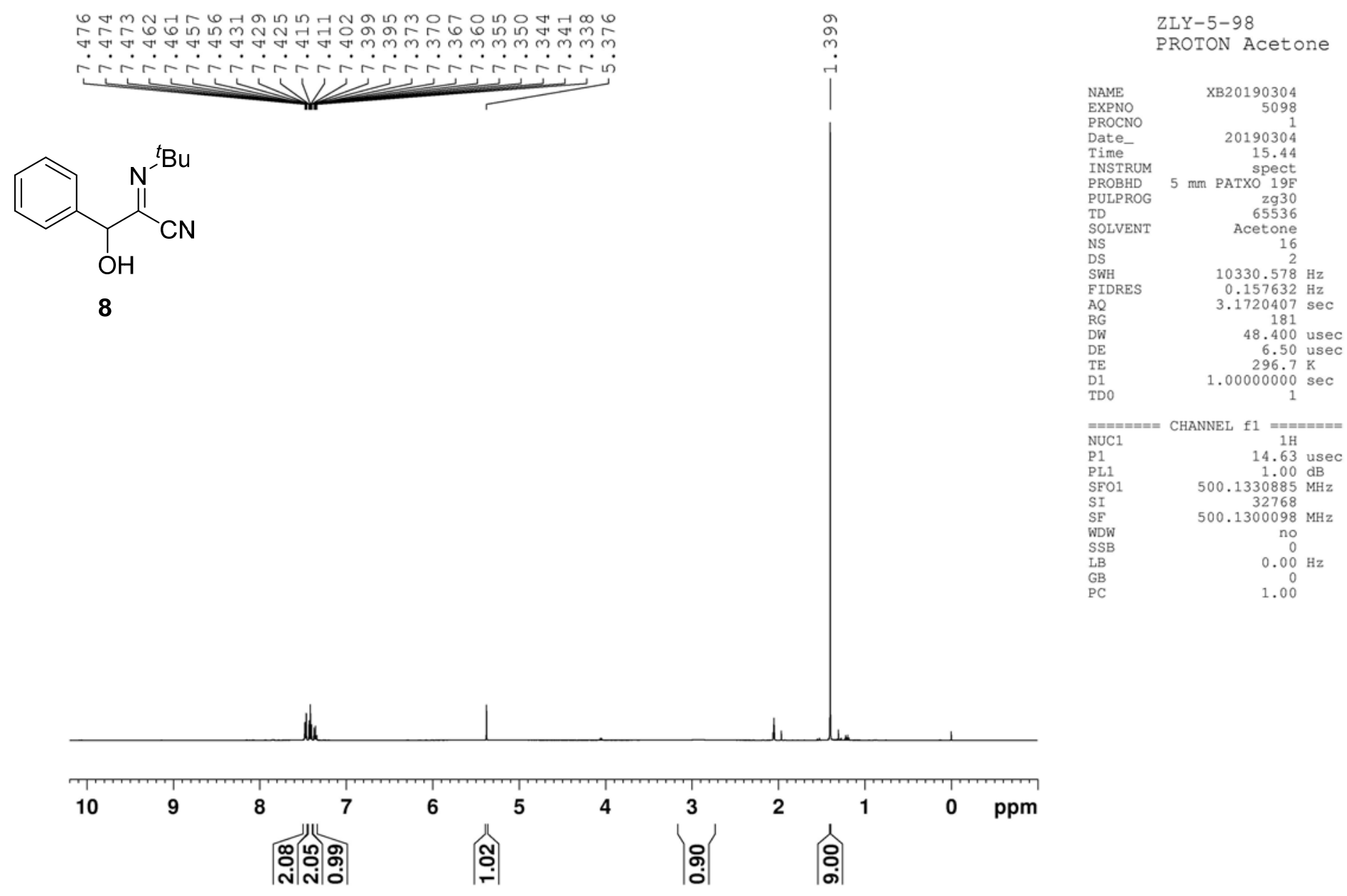




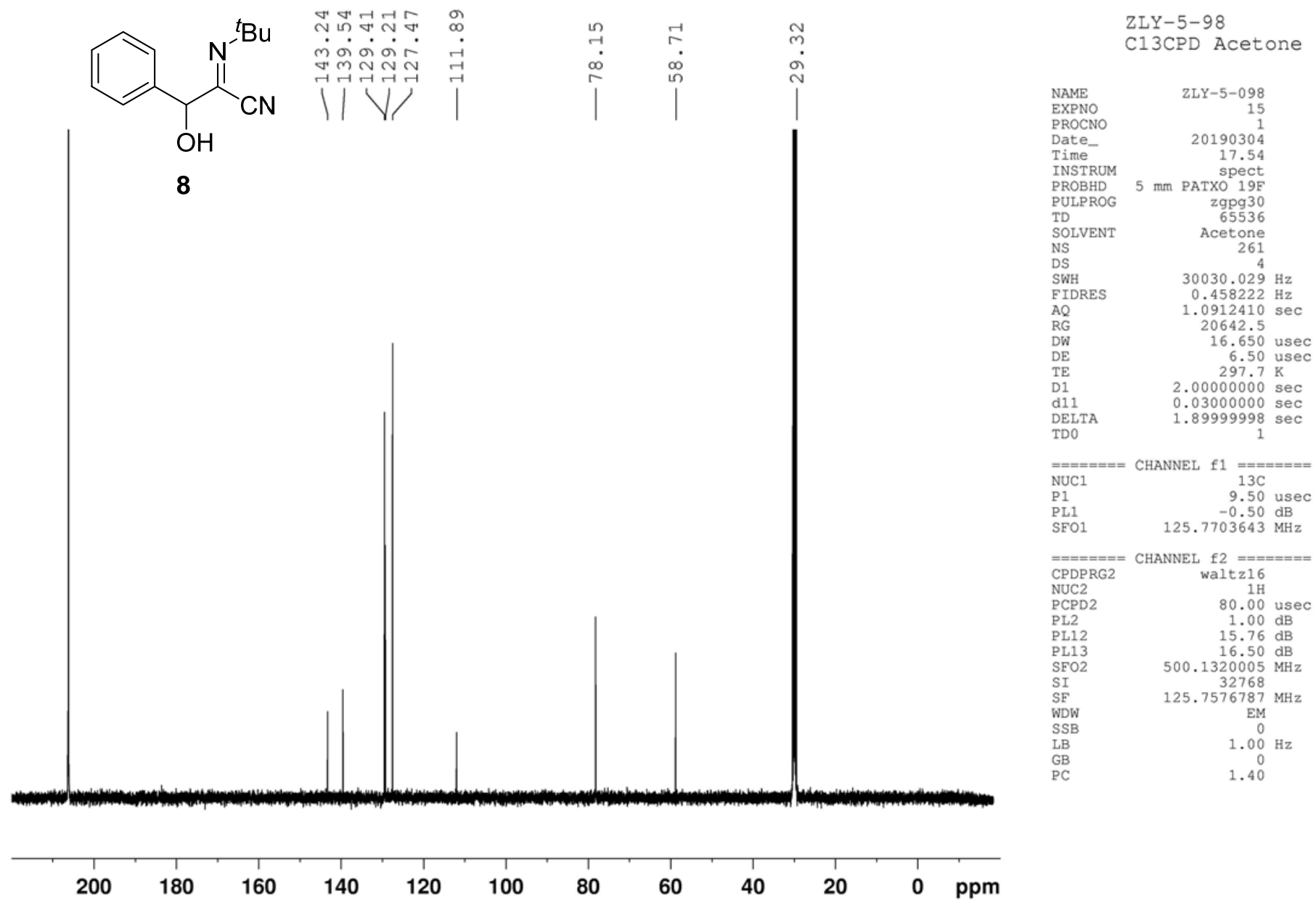




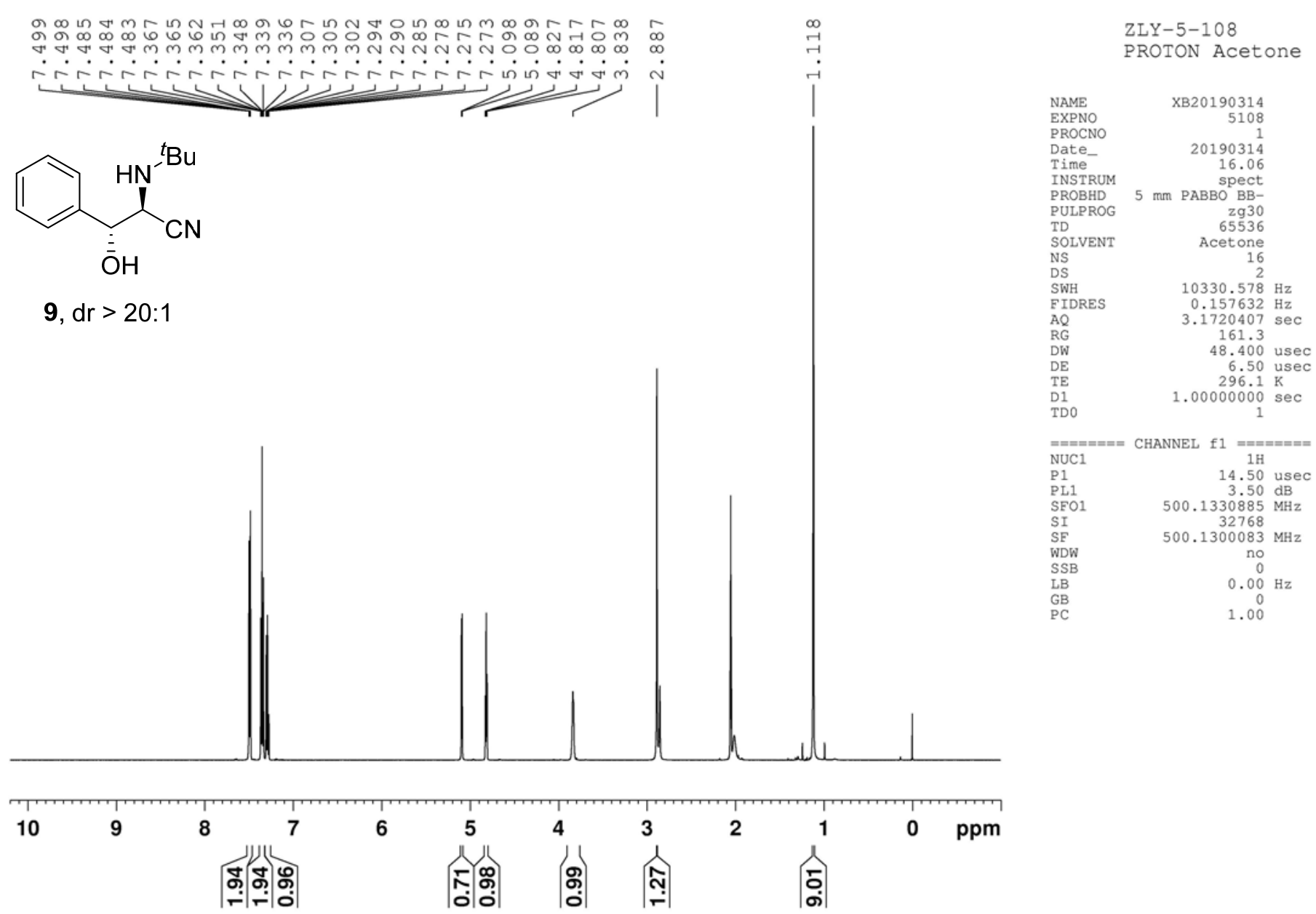




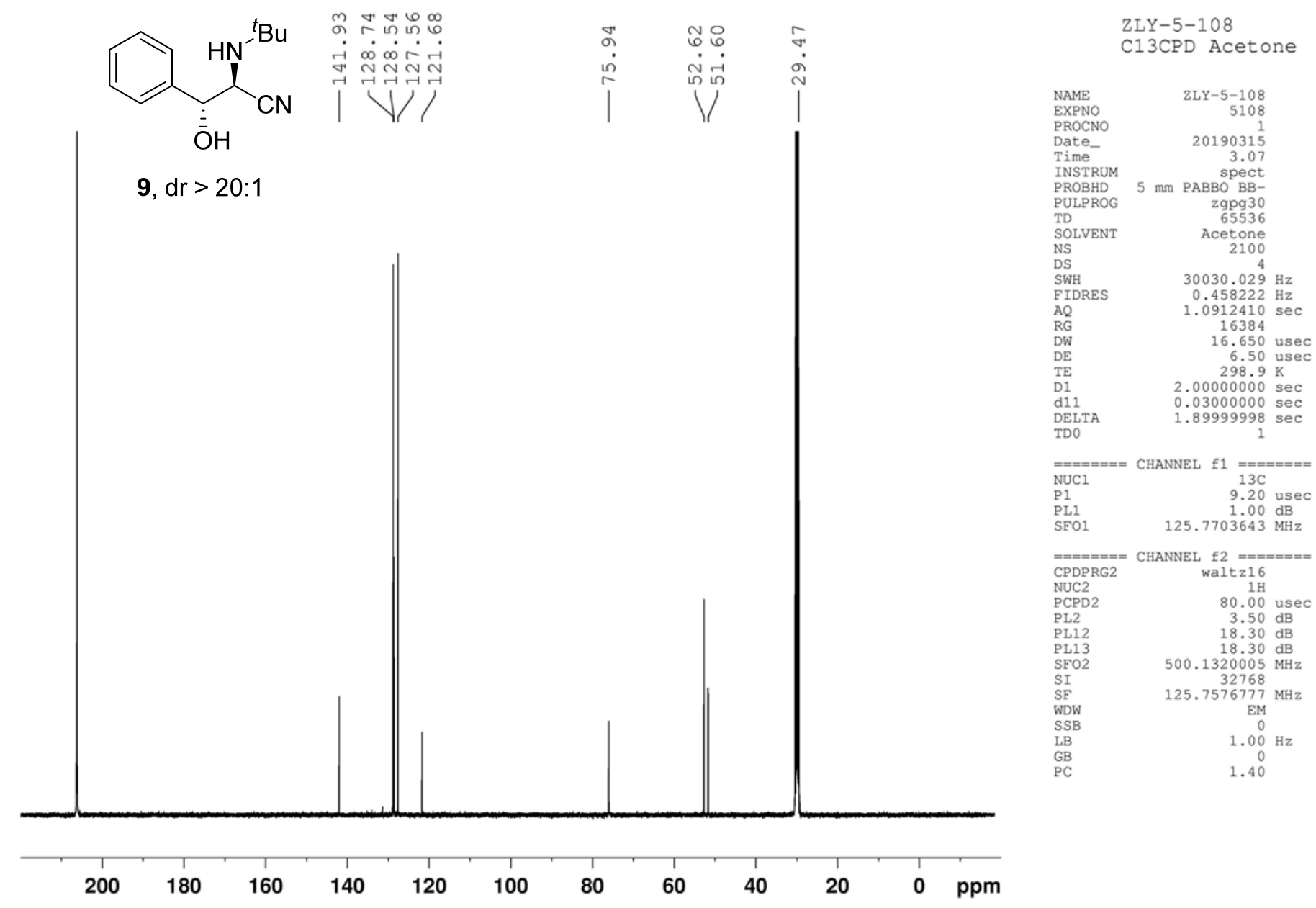




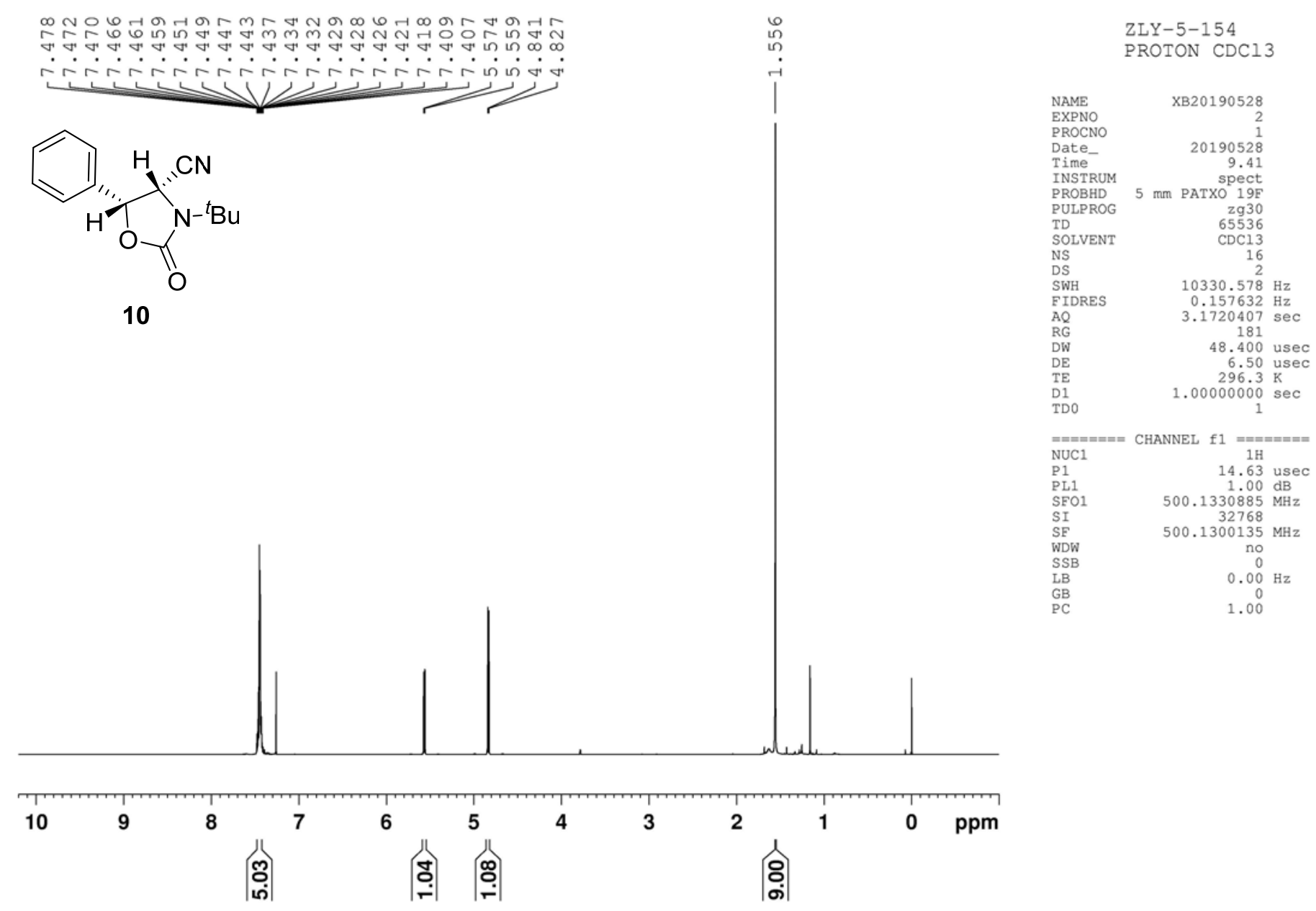




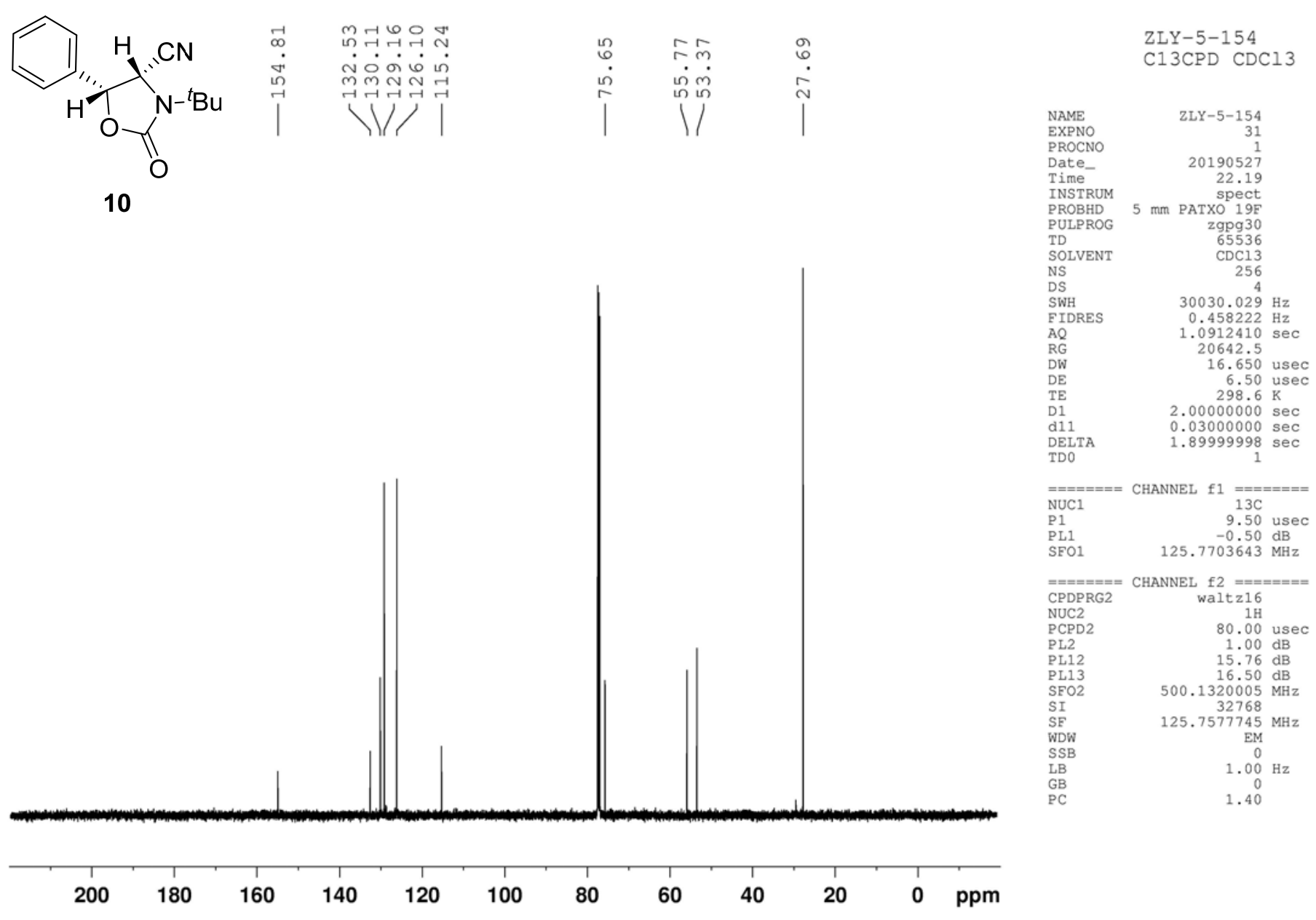




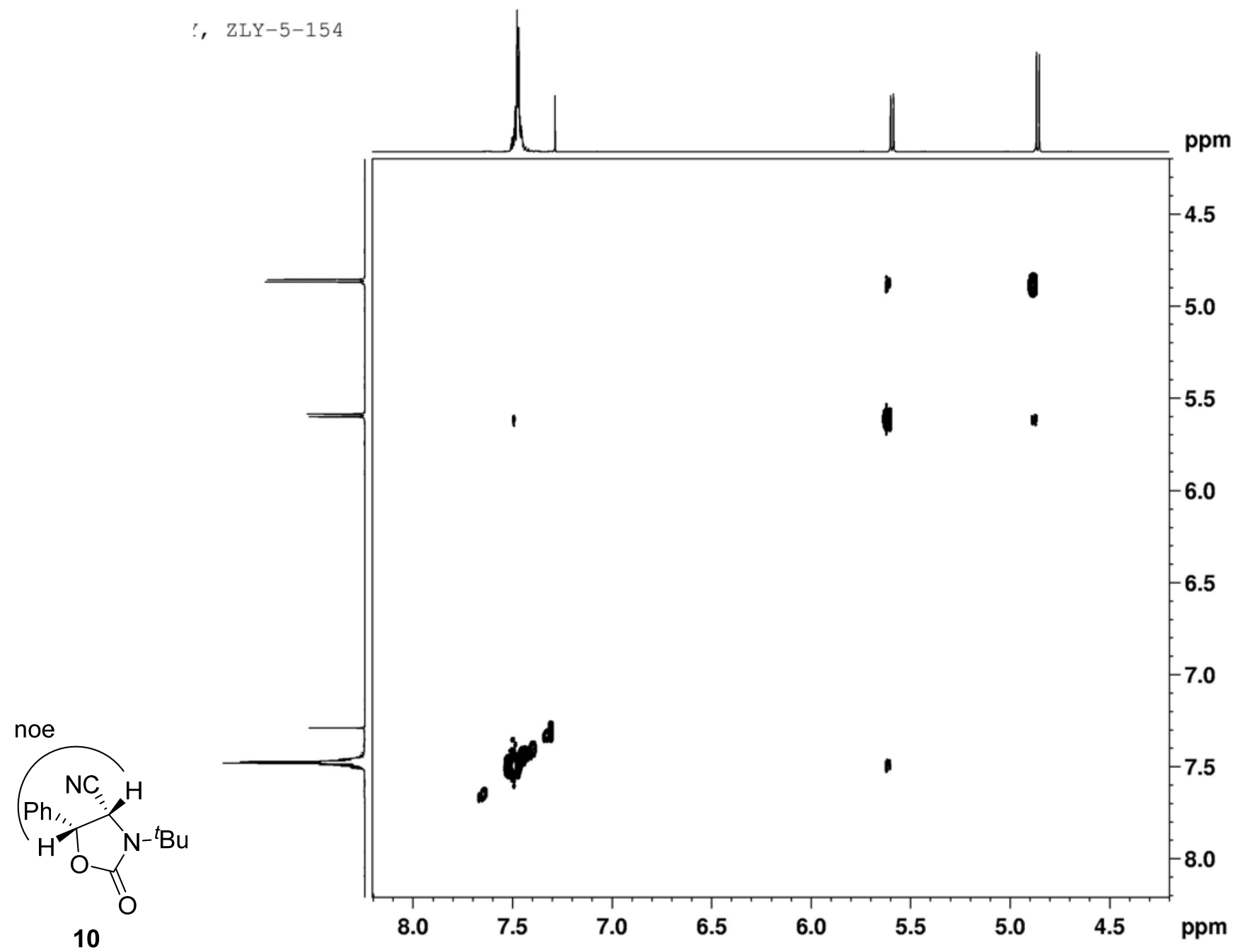

